UNIVERSITY OF SALAMANCA

DOCTORAL THESIS

\title{
Experimental studies for generation, transport and applications of ultra-fast laser driven $\mathrm{X}$-ray sources
}

Author:

Ghassan ZERAOULI
Thesis Directors:

Dr. G. Gatti

Dr. J.A. Pérez Hernández

USAL Tutor:

Prof. L. Volpe

A thesis submitted in fulfilment of the requirements for the degree of Doctor of Philosophy

September 9, 2020
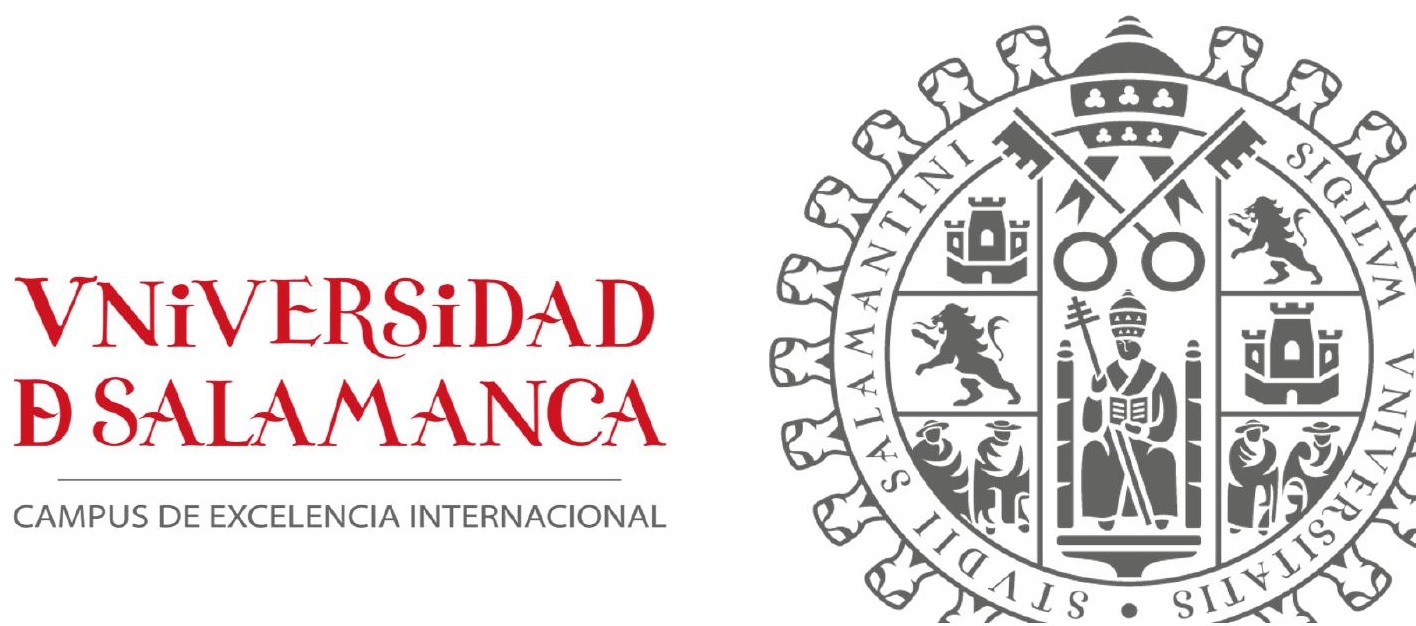



\section{Declaration of Authorship}

I, Ghassan ZERAOULI, declare that this thesis titled, "Experimental studies for generation, transport and applications of ultra-fast laser driven X-ray sources" and the work presented in it are my own. I confirm that:

- This work was done wholly or mainly while in candidature for a research degree at this University.

- Where any part of this thesis has previously been submitted for a degree or any other qualification at this University or any other institution, this has been clearly stated.

- Where I have consulted the published work of others, this is always clearly attributed.

- Where I have quoted from the work of others, the source is always given. With the exception of such quotations, this thesis is entirely my own work.

- I have acknowledged all main sources of help.

- Where the thesis is based on work done by myself jointly with others, I have made clear exactly what was done by others and what I have contributed myself.

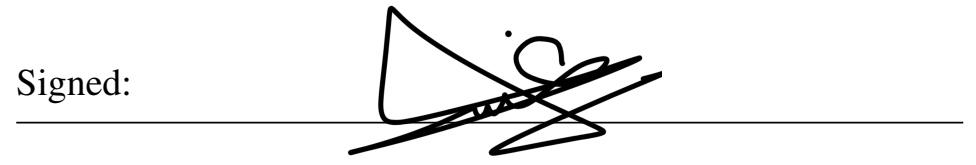

Date: $\quad$ Salamanca, 09-09-2020 



\section{Acknowledgements}

I would like first, to thank the director of CLPU, Prof. Luis Roso, for being by my side since the beginning of my adventure at CLPU, for trusting in my abilities and for providing all the necessary instrumentation needed for my investigations at CLPU. Also, I would like to thank you and the CLPU facility for the financial support to the experiments I've carried outside CLPU, and also for the important conferences and schools I have participated to during these years at CLPU. To you, and to all CLPU team, thank you.

I would also take this opportunity to thank my thesis tutor, Prof. Luca Volpe, who guided me since the first day I came to CLPU. I would like to thank you so much, for teaching me and for involving me in different research activities during my thesis. Your contribution to this work was enormous. I would like to thank you for being both, a great leader and a great person too.

I would like to thank my two PhD directors Dr. Jose Antonio Pérez-Hernández and Dr. Giancarlo Gatti. With you, Jose, we made so many experiments where you were essential. You have shown me, more than once, how one can count on you at work and outside too. I'm also grateful to my boss, Giancarlo, for your supervision and care in what concerns my multidisciplinary education by participating to schools, conferences, and experiments. You were fundamental for this work and I learned so much from you too.

I had the chance to work with one of the greatest physicists, Prof. Robert Fedosejevs. You were present in the laboratory, in the office and behind your screen, answering late mails and all possible questions I could ever made during the last five years with the same motivation and love for science. I learned so much from you, and I wish to continue doing so after my PhD too. Thank you.

I would like to thank Prof. Dimitri Batani for supervising me before I join CLPU and also for counting on me later, to manage many experimental activities either at CLPU or at other high power laser facilities during the period of my thesis. Thank you for being such a great professor and amazing person. I wish to continue collaborating with you.

Special thanks to my dear external collaborators and friends, with who I had the honour to work, in and outside CLPU, and from whom I learned a lot. Luca Antonelli, Franchesco Barbato and "Monsieur" Donaldi Mancelli, we made a great team by making X-ray phase contrast imaging great! On the other side, I would like also to thank my other external collaborator and friend, Andrew Longman, with who I have been involved in many experiments where we had fun and great results too.

I would like also to thank my dear team-mates, Sophia Malko, Carlos Salgado, Jon Imanol Apiñaniz, Diego De Luis, Michael Touati and also others, who left the group, but still ones of my best friends, Valeria Ospina and Xavier Vaisseau. Thank you for making both the office and laboratory very pleasant environments. I learned from all of you, and this thesis wouldn't have been possible without the contribution of each one of you. Also, I would like to thank other colleagues from other departments who became close friends such Jose Sagredo and Roberto Boyero who were there since the first day I came to CLPU and who helped me to adapt to Salamanca's life style. 
Finally, I would like to thank my dear parents, Mustapha Zeraouli and Adba Machan, for raising me in a such humble, amazing and loving family, together with the best sister one can ever have Rania Zeraouli. Even if in a certain moment of my life, the future was uncertain, you were there, supporting and trusting, unconditionally in me. You did huge sacrifices so that I could continue my studies far from home, believing in a better future for your son. There are no words, great enough to describe what you mean to me. Thank you for making me the man I am right now, I'll love you forever. Talking about family, I would also like to thank my life partner Edelweiss Hernández Oliva for being by my side for more than four years and for your unlimited cheering. I'm so glad I met you. 


\section{UNIVERSITY OF SALAMANCA}

\section{Abstract}

Centro de Laseres Pulsados (CLPU)

Doctor of Philosophy

\section{Experimental studies for generation, transport and applications of ultra-fast laser driven X-ray sources}

by Ghassan ZERAOULI

This thesis describes the physics of generation and transport of laser-driven x-ray sources and treats some of their related applications. X-ray emissions such fluorescence, bremsstrahlung and betatron driven by the interaction of focused ultra-short (30fs) and ultra-intense $\left(>10^{18} \mathrm{Wcm}^{-2}\right)$ laser pulses with under-dense and over-critical plasma has been described in the first part of this thesis. From one side, the theory of $\mathrm{x}$-ray transport has been well explored and exploited to design, build and test an adjustable, home-made, Kirkpatrick-Baez (KB) microscope in the Spanish Center for Pulsed Lasers (CLPU) to transport and focus specific spectral emission lines for both imaging and spectroscopy purposes, for High Repetition Rate (HRR) proton acceleration experiments. Alternative applications of the $\mathrm{KB}$ microscope such $\mathrm{x}$-ray transport for focused secondary x-ray sources are also presented.

On the other side, theoretically, a highly coherent $\mathrm{x}$-ray radiation source is needed to carry out high quality X-ray Phase Contrast Imaging (XPCI) experiments. Such X-ray sources can be only in XFEL (X-ray Free Electron Lasers) facilities. In this thesis, we have confirmed that focusing intense laser pulses into micrometer scale spots can also be used to carry out successful XPCI experiments. In other words, we have made possible, successful XPCI studies even with using broadband and incoherent $\mathrm{x}$-ray sources. Results from different experimental campaigns aiming for XPCI radiography using x-rays generated via Laser Wakefield Acceleration (LWFA) and other from pure bremsstrahlung are discussed at the end of this thesis.

Keywords: Kirkpatrick-Baez microscope, X-ray transport, X-ray Phase Contrast Imaging 



\section{Extracto (Spanish Abstract)}

En esta Tesis se presenta un estudio basado en la Física que describe la generación y transporte de fuentes de rayos $\mathrm{X}$ generados por láser, así como de algunas de sus aplicaciones más directas. En la primera parte de la Tesis se describe la generación de distintos tipos de radiación X, tales como la fluorescencia, la radiación de frenado (Bremsstrahlung) y la radiación Betatrón generadas cuando un pulso láser ultra-corto (30fs) y ultra-intenso $\left(>10^{18} \mathrm{~W} / \mathrm{cm}^{2}\right)$ se focaliza sobre un gas de átomos de de baja densidad generando un plasma en régimen de lo que se conoce como "under critical plasma". Por un lado se ha profundizado en el estudio de cómo "manejar" y focalizar los rayos X, una vez generados mediante la interacción laser-plasma. Para este propósito en el Centro de Láseres Pulsados (CLPU) se ha diseñado, construido y testado lo que se conoce como un microscopio Kirkpatrick-Baez (KB) para guiar y focalizar determinadas lineas de emisión espectral para aplicaciones en espectroscopia y en imagen de rayos $\mathrm{X}$ en experimentos de aceleración de protones a alta tasa de repetición de disparo.

Por otro lado, en experimentos de lo que se conoce como Radiografía por Contraste de Fase (XPCI, X-ray Phase Contrast Imaging) se necesitan rayos X altamente coherentes (al menos espacialmente) y este tipo de radiación en principio sólo era posible generarla mediante un Láser de Electrones Libres (XFEL, X-Free Electron Laser). En esta Tesis se ha confirmado que focalizando un láser intenso en un spot a escala micrométrica se pueden realizar satisfactoriamente Radiografías por Contrate de Fase. En otras palabras se han llevado a cabo, con éxito, estudios de Radiografía de Contraste de Fase usando incluso fuentes de rayos $\mathrm{X}$ de ancho de banda incoherente. Se presentan y se discuten resultados obtenidos en diferentes campañas experimentales orientadas hacia Radiografías de Contraste de Fase usando los rayos X generados mediante el proceso conocido como Laser Weakfield Acceleration (LWFA) así como otras obtenidas por otras vías, tales como las obtenidas mediante un proceso puramente de Bremsstrahlung.

Palabras clave: Microscopio Kirkpatrick-Baez, Transporte de rayos X, Imagen por rayos X mediante Contraste de Fase 



\section{Contents}

Declaration of Authorship iii

$\begin{array}{ll}\text { Acknowledgements } & \text { v }\end{array}$

Abstract vii

Extracto (Spanish Abstract) $\quad$ ix

Motivation and aim of the thesis $\quad$ Xv

Publications and research activities $\quad$ xvii

I Laser matter interaction 1

I.1 Laser technology . . . . . . . . . . . . . . . . . . . . . . . . 1

I.1.1 Gaussian beams and general properties . . . . . . . . . . . . 1

I.1.2 Laser amplification: CPA technology . . . . . . . . . . . . . 3

I.2 Laser-plasma processes . . . . . . . . . . . . . . . . . . 5

I.2.1 Introduction to plasma $\ldots \ldots \ldots \ldots \ldots$

I.2.2 Ionisation processes . . . . . . . . . . . . . . . 7

I.2.3 Characteristic quantities of plasmas . . . . . . . . . . . . . . 9

I.2.3.a Plasma quasi-neutrality . . . . . . . . . . . . 9

I.2.3.b Landau length . . . . . . . . . . . . . . . . . . . 9

I.2.3.c Electronic density distribution and Debye length . . . . . . . 10

I.2.3.d Plasma oscillation: electronic plasma frequency . . . . . . . . 12

I.2.3.e Critical density . . . . . . . . . . . . . . . 13

I.2.4 Motion of an electron in a laser field . . . . . . . . . . . . . . . . 15

I.2.5 The ponderomotive force . . . . . . . . . . . . . . . . 18

I.2.6 Electron acceleration in the underdense plasma: Laser Wakefield Accel-

eration LWFA . . . . . . . . . . . . . . . . . . 22

I.2.6.a Electron trapping and acceleration in plasma waves . . . . . 22

I.2.6.b Electron injection schemes in plasma wakes . . . . . . . . 25

I.2.6.c Acceleration limits . . . . . . . . . . . . . . . . 26

I.2.7 Electron acceleration in the over dense plasma . . . . . . . . . . . . 27

I.2.7.a Target Normal Sheath Acceleration (TNSA) principle . . . . . 27

I.2.7.b Introduction to fast electrons . . . . . . . . . . . . 28

II Laser driven X-ray sources $\quad 31$

II.1 Types of X-ray sources . . . . . . . . . . . . . . . . . . . . . . 34

II.1.1 $\mathrm{K} \alpha$ emission . . . . . . . . . . . . . . . . . 34 
II.1.1.a Generation . . . . . . . . . . . . . . . . . . . 34

II.1.1.b $\mathrm{K} \alpha$ emission cross section . . . . . . . . . . . . . . . . 35

II.1.1.c $\mathrm{K} \alpha$ photon number . . . . . . . . . . . . . . . 38

II.1.2 Bremsstrahlung emission . . . . . . . . . . . . . . . . . . . . . . . . . 39

II.1.3 Betatron radiation . . . . . . . . . . . . . . . . . . . 41

II.1.3.a Electron oscillations in the ion cavity . . . . . . . . . . . 41

II.1.3.b Radiation properties . . . . . . . . . . . . . . . . . . . . 42

II.2 X-ray transport optics and measurement . . . . . . . . . . . . . . . . . . . 44

II.2.1 X-ray matter interaction theory . . . . . . . . . . . . . . . . . . . . . . . . . . . . 44

II.2.2 Bragg crystals . . . . . . . . . . . . . . . . . . . 50

II.2.2.a Bragg crystals for X-ray spectroscopy _. . . . . . . . 51

II.2.2.b Bragg crystals for X-ray imaging . . . . . . . . . . . . . 55

III The adjustable Kirkpatrick-Baez microscope: design, building and testing 57

III.1 Theory and design of the KB microscope . . . . . . . . . . . . . . . . . . . 57

III.1.1 Design of the Kirkpatrick-Baez microscope . . . . . . . . . . . . . . . . 57

III.1.1.a General imaging relations . . . . . . . . . . . . . . 58

III.1.1.b KB microscope acceptance . . . . . . . . . . . . . . . . . 59

III.1.1.c Focal spot of the KB microscope . . . . . . . . . . . . . . . . 60

III.1.2 The "Adjustable" KB microscope . . . . . . . . . . . . . . . . . . . . . 60

III.1.2.a Motivation, inputs and conception . . . . . . . . . . . 60

III.1.2.b Mechanical design and simulations . . . . . . . . . . . . . 62

III.1.2.c Acceptance of the adjustable KB microscope . . . . . . . . . . 65

III.1.2.d KB microscope alignment procedure . . . . . . . . . . . 66

III.2 Optical characterisation of the KB microscope . . . . . . . . . . . . . . . . 68

III.2.1 Characterisation of the mirrors flatness _ . . . . . . . . . . . 68

III.2.2 Characterisation of the focal spot . . . . . . . . . . . . 71

IV Experimental studies $\quad \mathbf{7 5}$

IV.1 High intensity laser facilities . . . . . . . . . . . . . . . . . . 75

IV.1.1 CLPU laser facility . . . . . . . . . . . . . . . . . . 75

IV.1.2 PHELIX laser of GSI facility . . . . . . . . . . . . . . . 76

IV.2 X-ray detection . . . . . . . . . . . . . . . . . . . 77

IV.2.1 Passive detectors: Imaging plates . . . . . . . . . . . . . . 78

IV.2.2 Active detectors: X-ray CCD cameras . . . . . . . . . . . . . . . . 80

IV.3 Testing and validation of the KB microscope . . . . . . . . . . . . . . . . 80

IV.3.1 The KB microscope for $\mathrm{x}$-ray imaging . . . . . . . . . . . . 80

IV.3.1.a Proton acceleration experiment 1 (L.Volpe 2018) . . . . . . . 80

IV.3.1.b Strong shocks generation experiment 1 (D. Batani 2018) . . . . 84

IV.3.1.c Proton acceleration experiment 2 (R. Fedosejevs 2019): KB microscope versus curved Bragg crystal _. . . . . . . . . 90

IV.3.2 The KB microscope for focused secondary X-ray sources . . . . . . . . . 94

IV.4 X-ray based applications: X-ray Phase Contrast Imaging XPCI . . . . . . . . . . 95

IV.4.1 Principles for XPCI . . . . . . . . . . . . . . . . . . . . . . 95

IV.4.2 XPCI experiment 1 (R. Fedosejevs 2017) . . . . . . . . . . . . . 97

IV.4.3 XPCI experiment 2 (L. Antonelli 2017) . . . . . . . . . . . . . . . 100 
VI Resumen de la thesis (Spanish Summary) 



\section{Motivation and aim of the thesis}

Linear accelerators, new generation synchrotrons and XFEL's (X-ray Free Electron Laser) are the only facilities on the planet to deliver high brilliance x-ray sources with high accuracy (intensity, energy spread, nano-scale etc...). Due to the few number of these high dimension and high cost facilities, laser based accelerators are a promising alternative for particles acceleration and x-ray generation. Indeed, it has been theoretically and then experimentally confirmed that plasmas can sustain extreme electrical fields (up to $1 \mathrm{GeV} / \mathrm{cm}$ ). Thus, controlling the generation (via both, lasers and high repetition rate targets development) and the application of such fields for electron acceleration permits the creation of relativistic electron populations responsible of the emission of ultra-short $\mathrm{x}$-ray pulses. This modern technology is very promising and has many applications in different fields. Mastering the generation and transport of these laser-driven $\mathrm{x}$ ray sources is improving continuously, making several applications possible (and competitive; versus synchrotron and XFEL's). All these challenges have stimulated this work from which we identify the following aims:

- Development of ultra-short laser-driven x-ray sources in under-dense and over-critical density plasmas.

- Design, building and testing of an optic operating in the x-ray range and performing at high repetition rate with an automatic adjustability control for spectroscopy and imaging for laser-plasma interaction experiments.

- Investigating the X-ray Phase Contrast Imaging (XPCI) technique using two types of incoherent laser driven $\mathrm{X}$-ray sources at different high power laser facilities. 



\section{Publications and research activities}

I have participated during my PhD to many internal (in CLPU) and external (other international facilities) experiments. These research activities have led to the generation of an important number of scientific publications (articles in peer reviewed journals, posters and oral talks). The list of these scientific communications is shown in the following sections.

\section{Articles: (7)}

- A. Longman, C. Salgado, G. Zeraouli, Jon I. Apinaniz, J. A. Perez-Hernandez, M. Khairy Eltahlawy, L. Volpe, and R. Fedosejevs. "Off-axis spiral phase mirrors for generating high-intensity optical vortices". Optics Letters, Vol. 45, No. 8, April 2020. Contribution: Beam transport, installation of the experimental setup and data acquisition.

- J. Pasley, G. Andrianaki, J. I. Apiñaniz, A. Baroutsos, D. Batani, E. P. Benis, A. Ciardi, D. Cook, M. D. Marco, V. Dimitriou, B. Dromey, I. Fitilis, G. Gatti, A. Grigoriadis, M. Huault, J. A. Perez Hernandez, E. Kaselouris, O. Klimo, M. Koenig, G. Koundourakis, M. Kucharik, J. Limpouch, R. Liska, C. Salgado Lopez, S. Malko, S. Olmos-Miguelanez, Y. Orphanos, V. Ospina, N. A. Papadogiannis, S. Petrakis, J. Psikal, M. Rico, M. S. Rivetta, M. J Rodriguez-Conde, J. J. Santos, M. Sinor, A. Skoulakis, I. Tazes, L. T. Pascual, M. Touati, C. Tsitou, P. Vachal, L. Volpe, J. Vyskocil, S. White, M. Yeung, G. Zeraouli, and M. Tatarakis. "Innovative education and training in high power laser plasmas (PowerLaPs) for plasma physics, high power laser matter interactions and high energy density physics: experimental diagnostics and simulations". High Power Laser Science and Engineering, Vol. 8, e9, February 2020. Personal contribution: oral presentation at the PowerLaPs training school on diagnostic techniques for HHR (High Repetition Rate), March 25th-29th, 2019, held at CLPU, Salamanca, Spain. Title of the talk: "X-ray diagnostics: Kirkpatrick-Baez microscope".

- $\quad$ F. Barbato, S. Atzeni, D. Batani, D. Bleiner, G. Boutoux, C. Brabetz, P. Bradford, D.

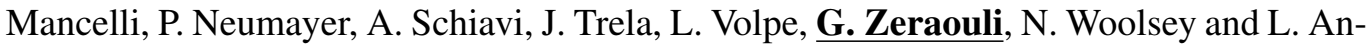
tonelli. "Quantitative phase contrast imaging of a shock-wave with a laser-plasma based $X$-ray source". Scientific Reports, vol 9, 18805, December 2019. Personal contribution: installation of the experimental setup, carrying out the experiment, data acquisition and partial analysis.

- C. Z. He, A. Longman, J. A. Perez-Hernandez, M. De Marco, C. Salgado, G. Zeraouli, G. Gatti, L. Roso, R.Fedosejevs and W. T. Hill. "Towards an in situ, full-power gauge of the focal-volume intensity of petawatt-class lasers". Optics Express, Vol. 27, Issue 21, pp. 30020-30030, October 2019. Personal contribution: partial installation of the experimental setup. 
xviii

- G. Zeraouli, G. Gatti, A. Longman, J. A. Pérez-Hernández, D. Arana, D. Batani, K. Jakubowska, L. Volpe, L. Roso, and R. Fedosejevs. "Development of an adjustable Kirkpatrick-Baez microscope for laser driven $x$-ray sources". Review of Scientific Instruments, 90, 063704, June 2019. Personal contribution: experimental setup installation, data acquisition and analysis, writing of the manuscript.

- L. Antonelli, F. Barbato, D. Mancelli, J. Trela, G. Zeraouli, G. Boutoux, P. Neumayer, S. Atzeni, A. Schiavi, L. Volpe, V. Bagnoud, C. Brabetz, B. Zielbauer, P. Bradford, N. Woolsey, B. Borm and D. Batani. "X-ray phase-contrast imaging for laser-induced shock waves". Europhysics Letters, EPL 125 35002, March 2019. Personal contribution: installation of the experimental setup, carrying out the experiment, data acquisition and partial analysis.

- L. Volpe, R. Fedosejevs, G. Gatti, J. A. Pérez-Hernández, C. Mendez, J. Apiñaniz, X. Vaisseau, C. Salgado, M. Huault, S. Malko, G. Zeraouli, V. Ospina, A. Longman, D. De Luis, K. Li, O. Varela, E. Garcia, I. Hernandez, J. D. Pisonero, J. Garcia Ajates, J. M. Alvarez, C. Garcia, M. Rico, D. Arana, J. Hernandez-Toro, and L. Roso. "Generation of high energy laser-driven electron and proton sources with the 200 TW system VEGA 2 at the Centro de Laseres Pulsados". High Power Laser Science and Engineering, Vol 7, e25, January 2019. Personal contribution: installation of the experimental setup, carrying out the experiment, data acquisition and partial analysis.

\section{Conference proceedings: (3)}

- G. Claps, F. Cordella, D. Pacella, A. Romano, F. Murtas, D. Batani, O. Turianska, D. Raffestin, L. Volpe, G. Zeraouli, J.A. Perez-Hernandez and S. Malko. "Soft X-ray measurements with a gas detector coupled to microchips in laser-plasma experiments at VEGA-2". Journal of Instrumentation, Vol 15, February 2020. (3rd European Conference on Plasma Diagnostics (ECPD2019), Lisbon, Portugal). Personal contribution: Beam transport, installation of the experimental setup and carrying out of the experiment.

- $\quad$ M. Shahzad, L.R. Reid, R. Spesyvtsev, A. Maitrallain, G.K Holt, G. Zeraouli, W. Li, G. Vieux, E. Brunetti, S.M. Wiggins, G. Gatti, D. De Luis, L. Volpe, R. Fedosejevs, and D.A. Jaroszynski. "Characterization of a laser plasma accelerator $x$-ray source size using a Kirkpatrick-Baez microscope". Proc. SPIE 11036, Relativistic Plasma Waves and Particle Beams as Coherent and Incoherent Radiation Sources III, 110360Q, April 2019. (SPIE Optics + Optoelectronics, 2019, Prague, Czech Republic). Personal contribution: Theoretical study and design of the Kirkpatrick-Baez microscope.

- F. Barbato, D. Batani, D. Mancelli, J. Trela, G. Zeraouli, G. Boutoux, P. Neumayer, S. Atzeni, A. Schiavi, L. Volpe, V. Bagnoud, C. Brabetz, B. Zielbauer, P. Bradford, N. Woolsey, B. Bormi and L. Antonelli. "Propagation-based imaging phase-contrast enhanced imaging setup for single shot acquisition using laser-generated X-ray sources". Journal of Instrumentation, Vol14. March 2019. (5th International Conference Frontiers in Diagnostics Technologies (ICFDT), Rome, Italy). Personal contribution: installation of the experimental setup, carrying out the experiment, data acquisition and partial analysis. 


\section{Posters: (10)}

Note that only the posters where I am first author, were presented by me.

- G. Zeraouli, G. Gatti, A. Longman, J. A. Pérez Hernández, D. Arana, D. Batani, K. Jakubowska, L. Volpe, L. Roso and R. Fedosejevs. "Development of an adjustable Kirkpatrick-Baez microscope for laser driven x-ray sources at CLPU". Poster at the 3rd European Conference on Plasma Diagnostics (ECPD2019), Lisbon, Portugal, May 2019. Personal contribution: installation of the experimental setup, carrying out the experiment, data acquisition and analysis, poster writing.

- G. Claps, F. Cordella, D. Pacella, A. Romano, F. Murtas, D. Batani, O. Turianska, D. Raffestin, L. Volpe, G. Zeraouli, J.A. Perez-Hernandez and S. Malko. "Soft X-ray measurements with a gas detector coupled to microchips in laser-plasma experiments at VEGA-2". Poster at 3rd European Conference on Plasma Diagnostics (ECPD2019), Lisbon, Portugal, May 2019. Personal contribution: Beam transport, installation of the experimental setup and carrying out of the experiment.

- G. Claps, F. Cordella, D. Pacella, F. Murtas, D. Batani, O. Turianska, D. Raffestin, L. Volpe, G. Zeraouli, J.A. Perez-Hernandez, S. Malko, "Gamma rays detection in laser produced plasmas with Silicon C-MOS imager by means of trace analysis". Poster at 3rd European Conference on Plasma Diagnostics (ECPD2019), Lisbon, Portugal, May 2019. Personal contribution: Beam transport, installation of the experimental setup and carrying out of the experiment.

- I. Gallardo González, H. Ekerfelt, J. Björklund Svensson, G. Gatti, A. Gonoskov, D. Guenot, M. Hansson, M. Marklund, J. A. Pérez-Hernández, C. Salgado, E. Wallin, G. Zeraouli and O. Lundh."Electron acceleration and X-ray emission from interacting wakefields". Poster at IONS Scandinavia, Copenhagen, Denmark and Lund, Sweden. June 2018. Personal contribution: Beam transport, installation of the experimental setup and carrying out of the experiment.

- $\quad$ F. Barbato, S. Atzeni, D. Batani, D. Bleiner, G. Boutoux, C. Brabetz, P. Bradford, D. Mancelli, P. Neumayer, A. Schiavi, J. Trela, L. Volpe, G. Zeraouli, N. Woolsey and L. Antonelli "Experimental in-line Phase-Contrast Imaging (PCI) of a shock wave". Poster at the 10th International Conference on Inertial Fusion Sciences and Applications, IFSA, Saint Malo, France. September 2017. Personal contribution: installation of the experimental setup, carrying out the experiment, data acquisition and partial analysis.

- $\quad$ G. Zeraouli, R. Fedosejevs, G. Gatti, A. Longman, D. Arana, L.Volpe and L.Roso. "An adjustable Kirkpatrick Baez microscope as a diagnostic for laser driven x ray sources". Poster at the 2nd European Conference on Plasma Diagnostics, Bordeaux, France. April, 2017. Personal contribution: installation of the experimental setup, carrying out the experiment, data acquisition and analysis, poster writing.

- C. Salgado, A. Longman, S. Malko, G. Zeraouli, M. Huault, X. Vaisseau, J. A. PérezHernández, J. I. Apiñaniz, E. Garcia, O. Varela, C. Mendez, J. Hernandez-Toro, G. Gatti, L. Volpe, L. Roso and R. Fedosejevs. "Laser driven electrons and X-ray Betatron radiation generation at VEGA". Poster at the 13rd Direct Drive and Fast Ignition Workshop, 
Salamanca, Spain. March 2017. Personal contribution: installation of the experimental setup, carrying out the experiment, data acquisition.

- S. Malko, A. Longman, C. Salgado, J. Ajates, J. I. Apiñaniz, G. Gatti, I. Hernández, J. A. Pérez-Hernández, J.D. Pisonero, L. Roso, X.Vaisseau, L. Volpe, G. Zeraouli and R. Fedosejevs. "Study of ionization states dynamics of Warm Dense Aluminum". Poster at the 13rd Direct Drive and Fast Ignition Workshop, Salamanca, Spain. March 2017. Personal contribution: installation of the experimental setup, carrying out the experiment, data acquisition.

- $\quad$ G. Zeraouli, R. Fedosejevs, V. Pamo, G. Gatti and L. Roso. "Customized KirkpatrickBaez microscope for X-rays diagnostics". Poster at ELISS summer school, Dolní Břežany, Czech Republic. August 2016. Personal contribution: installation of the experimental setup, carrying out the experiment, data acquisition and analysis, poster writing.

- G. Zeraouli, A. Peralta Conde, F. Valle Brozas, J. I. Apiñaniz, A. V. Carpentier, G. Gatti, D. Bleiner, L. Masoudnia, L. Volpe and L.Roso. "Time and spatial-resolved measurements of plasma structures in laser produced plasmas at CLPU". Poster at the Quantum electronics summer school, Erice, Italy. July 2015. Personal contribution: participation to the installation of the experimental setup, poster writing.

\section{Oral contributions: (5)}

- G. Zeraouli, G. Gatti, A. Longman, J. A. Pérez-Hernández, D. Arana, D. Batani, K. Jakubowska, L. Volpe, L. Roso, and R. Fedosejevs. "Development of an adjustable Kirkpatrick-Baez microscope for laser-driven x-ray sources". Oral presentation, LaPlaSS, Laser Plasma Summer School, September 16th-20th, 2019, CLPU, Slamanca, Spain. Personal contribution: installation of the experimental setup, carrying out the experiment, data acquisition and analysis, oral presentation.

- G. Zeraouli, G. Gatti, A. Longman, J. A. Pérez-Hernández, D. Arana, D. Batani, K. Jakubowska, L. Volpe, L. Roso, and R. Fedosejevs. "X-ray diagnostics: Kirkpatrick-Baez microscope". Oral presentation, PowerLaPs training school on diagnostic techniques for HHR (High Repetition Rate), March 25th-29th, 2019, CLPU, Salamanca, Spain. Personal contribution: installation of the experimental setup, carrying out the experiment, data acquisition and analysis, oral presentation.

- L. Antonelli, F. Barbato, D. Mancelli, J. Trela, G. Zeraouli, G. Boutoux, P. Neumayer, S. Atzeni, A. Schiavi, L. Volpe, V. Bagnoud, C. Brabetz, B. Zielbauer, P. Bradford, N. Woolsey, B. Borm and D. Batani. "The Use of Phase-Enhanced X-Ray Radiography on High Energy Laser Facilities". Oral presentation, IFSA, Inertial Fusion Sciences and Applications, Osaka, Japan. September 2019. Personal contribution: installation of the experimental setup, carrying out the experiment, data acquisition and partial analysis.

- $\quad$ R. Fedosejevs, A. Longman, C. Salgado, G. Zeraouli, J. I. Apiñaniz, J. A. PérezHernández, M D. Marco, G. Gatti, L. Volpe, C. Z. R. He and W. T Hill. "MeV Electron and Betatron Production from Wakefield Interactions using Orbital Angular Momentum Laser Pulses". Oral presentation. Frontiers of Plasma Physics and Technology FPPT, 
Colombo, Sri Lanka. April 2019. Personal contribution: installation of the experimental setup, carrying out the experiment and data acquisition.

- A. Longman, C. Salgado, G. Zeraouli, J. I. Apiñaniz, J. A. Pérez-Hernández, M. De Marco, C. Z. He, G. Gatti, L. Volpe, W. T. Hill, and R. Fedosejevs. "Wakefield acceleration and betatron radiation driven by linearly polarized Laguerre-Gaussian orbital angular momentum laser pulses". Oral presentation. Super-Intense Laser-Atom Physics international workshop SILAP, Toronto, Canada. December 2018. Personal contribution: installation of the experimental setup, carrying out the experiment and data acquisition. 



\section{Chapter I}

\section{Laser matter interaction}

\section{Introduction}

Since its invention in the early 60's of the last century [Maiman, 1960], laser technology didn't stop improving. Indeed, lasers are being used more and more in different fields such as medicine [Masters, 2004], aerospace communications [Koepf et al., 2002], Inertial Confinement Fusion (ICF) [Glenzer et al., 2010] etc... All these laser applications wouldn't have been possible without the discovery and development of CPA (Chirped Pulse Amplification) technology [Strickland and Mourou, 1985] by the team of G. Mourou and D. Strickland in 1985 (2018 Nobel prize) which permitted to reach intensities above $10^{16} \mathrm{~W} / \mathrm{cm}^{2}$ with pulses in the ps, sub ps and fs range. Nowadays, scientists are starting to believe that laser-based accelerators could be the most reasonable alternative to mega infrastructures such as linear accelerators and synchrotrons in the future. Effectively, it has been proven, by both simulations [Tajima and Dawson, 1979, Joshi et al., 1984] and experiments [Rosenzweig et al., 1988, Mangles et al., 2004, Schlenvoigt et al., 2008, Malka, 2012], that electrons can be repetitively accelerated up to relativistic velocities (to Giga electron Volts) by carrying out LWFA (Laser Wakefield Acceleration) experiments. The general theory of plasmas needed, as a background, for the rest of the thesis is introduced in this chapter. We will start by introducing the technology of generation of ultra-short ultra-intense laser pulses. The main physical properties of laser generated plasmas are then described next. We will end up the chapter after presenting two important laser-driven mechanisms for particle acceleration separating two regimes of interaction, an under-dense regime for LWFA and an over-critical one TNSA (Target Normal Sheath Acceleration) [Passoni, 2010, Passoni et al., 2013].

\section{I.1 Laser technology}

\section{I.1.1 Gaussian beams and general properties}

In principle, to generate laser radiation, a closed optical cavity, formed by two mirrors coupled to an amplifier medium (or active medium), is needed. One of the cavity mirrors should be $100 \%$ reflecting, while the other one (called output mirror) is partially reflecting $(\sim 99 \%)$. The acronym 'LASER' stands for Light Amplification by Stimulated Emission of Radiation. The first step to generate a laser beam consists of exciting the atoms of the amplifier medium to generate a 'population inversion'. The excited atoms will emit, isotropically, photons of a specific energy, 
corresponding to the difference between two energy levels (excited and fundamental). For a given cavity length $L$, only the stationary waves for a given wavelength $\lambda$ and corresponding to discrete longitudinal modes of the cavity can oscillate (the non stationary waves just vanish). The modes of frequency $v_{q}=q . c / 2 L$ (where $q$ is a positive integer such $q \in \mathbb{Z}$ ) are called normal modes of the cavity. In addition to the so called 'stability condition' $\left(0<\left(1-L / R_{1}\right)\left(1-L / R_{2}\right)<1\right.$, where $R_{1,2}$ is respectively the radii of curvature for the mirror 1 and 2 of the cavity required for build up of the modes). One can obtain laser effect only if the total losses are less than or equal to the cavity gain. It is important to stress that the photons generated by lasers have all the same properties such as the phase $\varphi$, direction, polarisation and the central wavelength $\lambda_{L}$. Laser cavities are generally designed to let out only the electromagnetic waves containing the fundamental transverse mode. This one is described by a Gaussian in the space domain. The following mathematical expression Eq.I.1 of the electric field amplitude $E$ is a solution to the paraxial Helmoltz equation [Bandres and Gutiérrez-Vega, 2004]. Considering that the electromagnetic wave is propagating along the positive, $z$, direction and polarized in the, $x$, direction, $E$ is written:

$$
\boldsymbol{E}(r, z)=E_{0} \frac{w_{0}}{w(z)} \exp \left(\frac{-r^{2}}{w(z)^{2}}\right) \exp \left(-i\left(k z+k \frac{r^{2}}{2 R(z)}\right)-\psi(z)\right) \boldsymbol{x}
$$

Here $r$ stands for the radial distance from the centre axis of the beam. $i$ is the imaginary unit. $k=2 \pi / \lambda$ is the wave number for a wavelength $\lambda . E_{0}$ is the electric field amplitude and phase at the origin at time $0\left(E_{0}=E(0,0)\right) . w(z)$ is the radius at which the field amplitude falls to 1/e of its axial value (this corresponds to where the intensity values fall to $1 / e^{2}$ of the axial values). $w_{0}=w(0)$ is the waist radius. $w(z)$ can be written as a function of the beam waist radius $w_{0}$ as

$$
w(z)=w_{0} \sqrt{1+\left(\frac{z}{z_{R}}\right)^{2}}
$$

Where $z_{R}$ is the Rayleigh length $\left(z_{R}=\pi w_{0}^{2} / \lambda_{L}\right)$ and $\psi(z)=\arctan \left(z / z_{R}\right)$ is the Gouy phase at $z . R(z)$ is the radius of curvature of the beam's wavefronts at $z$ and is written

$$
R(z)=z\left[1+\left(\frac{z_{R}}{z}\right)^{2}\right]
$$

The intensity distribution $I(r, z)$ of a Gaussian beam is given by the time average of the energy flux of the electromagnetic field and is represented by the Poynting vector $S(r, t)=E(r, t) \times$ $\boldsymbol{B}(\boldsymbol{r}, t) / \mu_{0}$. Where $\boldsymbol{B}$ is the magnetic field and $\mu_{0}$ is the vacuum permeability. So $I(\boldsymbol{r})=<$ $S(r, t)>=c \varepsilon_{0}\left|E_{0}\right|^{2}$. We can then express $I(r, z)$

$$
I(r, z)=I_{0}\left(\frac{w_{0}}{w(z)}\right)^{2} \exp \left(\frac{-2 r^{2}}{w(z)^{2}}\right)
$$

Where $I_{0}=c \varepsilon_{0}\left|E_{0}\right|^{2}$ is the intensity at the centre of the beam. The resultant $I(r, z)$ around the beam waist region is shown in Fig.I.1.

Gaussian beams have many interesting properties such as keeping the same transverse Gaussian intensity profile for all $z$ positions.

A laser pulse can be described as the product of two terms in the temporal domain. The first one is oscillating with a central angular frequency $\omega_{L}=2 \pi c / \lambda_{L}$. The second term describes 

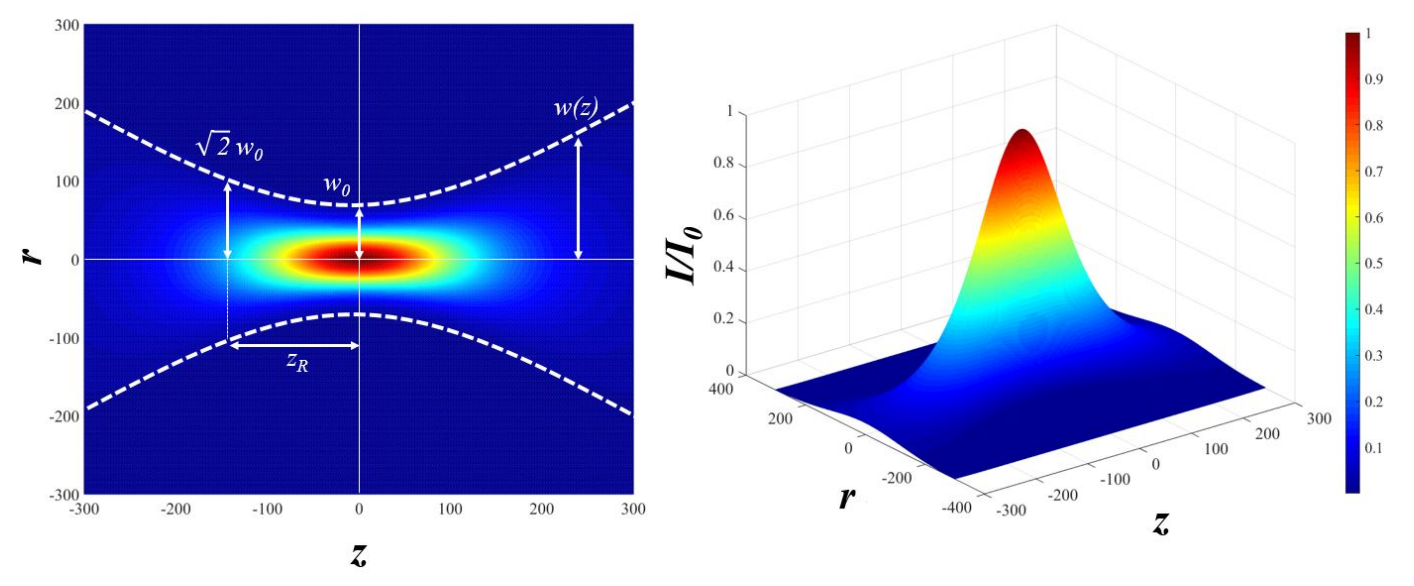

FIGURE I.1: Parameter definition for Gaussian beam profiles (left). Spatial intensity distribution of a Gaussian beam (right). The dashed line corresponds to the beam radius position.

the slowly varying envelope (often described by a Gaussan function) and represents the temporal shape of the pulse. The laser pulse in the time domain is then written as

$$
E_{L}(t)=E_{0} \exp \left[-2 \ln (2) \frac{t^{2}}{\tau_{L}^{2}}\right] \exp \left(-i \omega_{L} t\right)
$$

Where $\tau_{L}$ is the pulse duration at FWHM.

\section{I.1.2 Laser amplification: CPA technology}

Generating laser pulses of a few milijoules in the picosecond range has many applications in industry, biology, medicine, etc... However, the intensity generated with these lasers is still insufficient for some research fields of physics. For example, to reach the thermonuclear fusion conditions or to accelerate particles to relativistic speeds, intensities above $10^{16} \mathrm{~W} / \mathrm{cm}^{2}$ are needed. Logically, the first idea to come in mind would be to simply amplify the generated laser pulses up to whatever conditions/parameters required. The problem faced in this case is that, physically, one cannot amplify a laser pulse to infinite intensities. In reality, all materials used for laser amplification saturate at specific thresholds. In addition, one also generate non-linear effects such as Kerr lensing if the laser intensity is above some characteristic limits. Indeed, the index of refraction of materials $n$ can be written as a function of linear and non-linear refraction indexes, respectively $n_{0}$ and $n_{N L}$, and is written

$$
n=n_{0}+n_{N L} I_{L}
$$

Here $I_{L}$ stands for the laser intensity. When $I_{L}$ reaches a specific value, the non-linear term $\left(n_{N L} I_{L}\right)$ of the refraction index becomes important. Knowing that the spatial distribution of main laser pulses is Gaussian (where laser intensity is higher in the centre and decreases radially, as seen in Fig.I.1), implies that the central part of the beam will experience a higher index of refraction than the low intensity region. Consequently, this will lead to a beam focusing effect (from where the term of Kerr 'lens' comes from) as seen in Fig.I.2. 


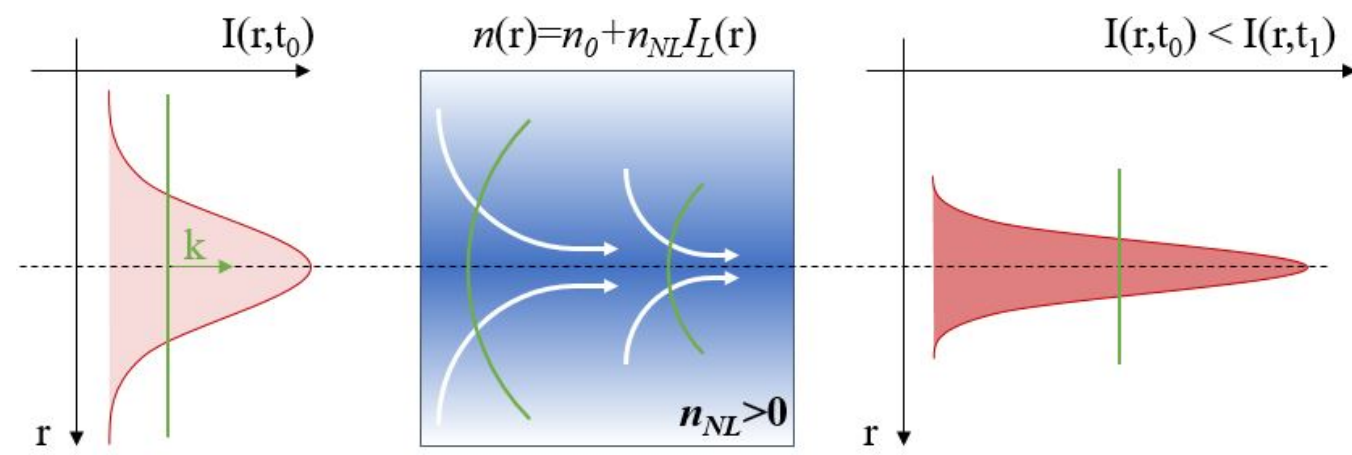

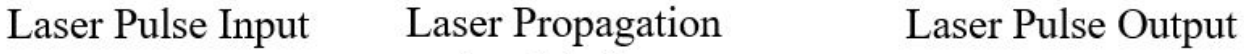 in a Medium}

FIGURE I.2: Illustration of the Kerr lensing effect. The laser pulse intensity profile is plotted in red colour. The amplifier medium is represented in blue. The variation of the blue colour is due to the increase of the refractive index at the centre (due to the beam intensity profile). In green we can appreciate the beam wavefront evolution.

Let us consider a laser beam of intensity $I_{L}$ propagating in an amplifier medium. The Kerr effect can occur if $I_{L}$ is sufficiently high to create non linear effects. The increase in the refractive index at beam center leads to a lens effect causing the beam to focus and even reach higher intensities. At some point the intensity can become high enough to start ionising the medium. If the electron density becomes higher than the critical density $n_{c}$ (for the laser's wavelength, see more details further in this chapter), the laser can no more propagate and the totality of the beam is diffracted. The refraction index of the plasma created $\eta_{p}$ can be written according to [Gibbon, 2005] as following

$$
\eta_{p}(z, t)=\left(1-\frac{n_{e}(z, t)}{n_{c}}\right)^{\frac{1}{2}}
$$

Here $n_{e}$ is the electron density. Reaching $n_{c}$ means that a plasma has been generated, which means that a permanent damage has been created in the optics. Consequently, this will have important effects on modifying the beam wavefronts. $B$-integral is a way of measuring beam distortions. It represents the value of the total distortions accumulated by the laser wavefront during its propagation. In other terms, $B$-integral, represents the measurement of non-linear phase shift of light. The latter should be preferably minimized in a optical systems and is given by

$$
B=\frac{2 \pi}{\lambda_{L}} \int^{Z} n_{N L} I_{L}(z) d z
$$

A propagating laser beam in an amplifier medium will experience then successive focusing and defocusing effects which dramatically limits the amplification. Direct-amplification to high intensities becomes impossible due to the non-linear effects and so, a new beam amplification scheme is needed. 
Facing this challenge, the group of G.Mourou [Strickland and Mourou, 1985] proposed and developed a new technique called CPA (see Fig.I.3), which stands for Chirped Pulse Amplification and which consists of amplifying short laser pulses of $\mathrm{nJ}$ of energy and fs duration, to ultra high energy pulses containing up to tens of Joules in a few femtoseconds. CPA basically relies on three steps:

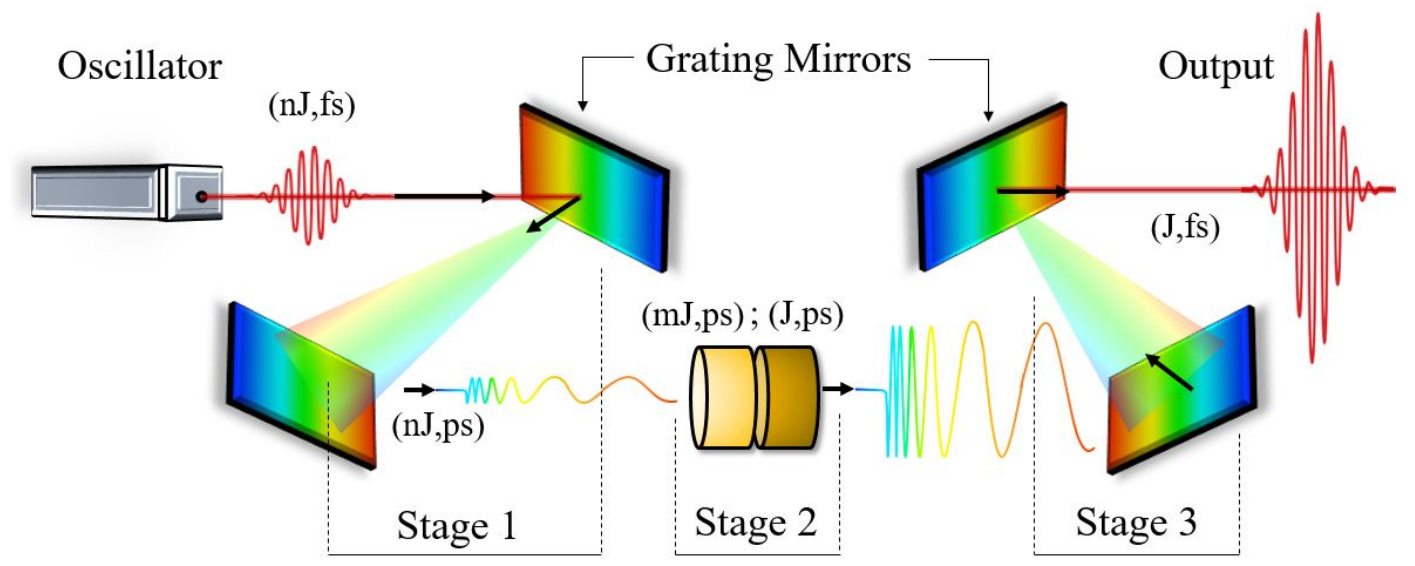

FIgURE I.3: Scheme for the principle of Chirped Pulse Amplification.

- Stage 1: The goal of this step is to reduce strongly the beam intensity to avoid non-linear effects during the amplification. This stage consists mainly of stretching temporally the beam using a set of grating mirrors. These optics are specially designed in such way the modes of different frequencies, contained in the seed pulse, are diffracted at different angles and follow different paths. The input seed pulse is characterised by a low energy (in the $\mathrm{nJ}$ range) and a short duration (few femtoseconds). The pulse exits the first stage with a duration in the tens to hundreds picosecond range.

- Stage 2: Temporally stretched, the amplification procedure can take place with no risks of non-linear effects. Energy gain of the order of $10^{9}$ can be safely reached using multipass amplifiers. The output pulse at this stage are in the range of hundred of ps and with energies of the order of tens of Joules for the most powerful lasers.

- Stage 3: Is the last step, and it consists of the temporally compressing of the beam (inverse of stage 1). The grating are oriented in such way the laser frequencies propagate on different paths and compensate the paths traversed in stage 1 . The pulse duration reduces from hundreds of picoseconds to few tens of femtoseconds. Thus, ultra high power laser pulses can be generated, reaching the Petawatt regime in some facilities (RAL, CLPU, DRACO, ELI, APOLLON, GEKKO, OMEGA ...).

\section{I.2 Laser-plasma processes}

\section{I.2.1 Introduction to plasma}

There exist many definitions of plasma and one of them describes it as the fourth state of matter where the latter is at least partially ionized. The word plasma means literally in ancient 
Greek "anything formed or molded" and was first introduced by Ivan Langmuir in the 1920's [Morozov, 2013]. The experiment shown in Fig.I.4 explains when a medium starts to be considered as plasma. Let consider a particle of one proton and one electron. To ionise the latter, a photon of energy $E_{a}$ higher than the binding energy of the electron $E_{B 1}$ is needed. The electron will be then emitted with an energy $\Delta E_{1}=E_{a}-E_{B 1}$ (see Fig.I.4.a). Now let's imagine, with the same photo-ionisation process, two photons of the same energy $E_{a}$, arriving with a small delay onto two particles composed of one proton and one electron each (see Fig.I.4.b). One electron will be ejected with $\triangle E 1=E_{a}-E_{B 1}$ while the second one will be affected by the electrostatic potential of the missing electron on the second particle and so will have an energy $\Delta E_{2}=E_{a}-E_{B 2}-\delta_{1}$ (where $\delta_{1}$ is the electrostatic potential of the particle number 1). One can think of a similar case but for a given number $\mathrm{N}$ of particles where the last electron ejected can no more leave the ion cloud and comes back to form a quasi neutral plasma which defines the first characteristic quantity of a plasma (see more in following section).

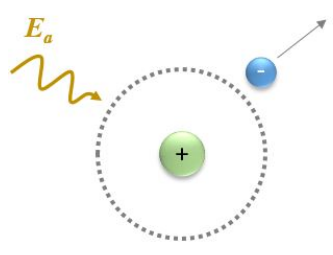

a.

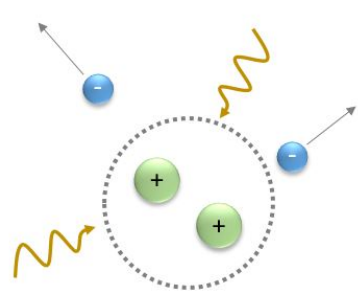

b.

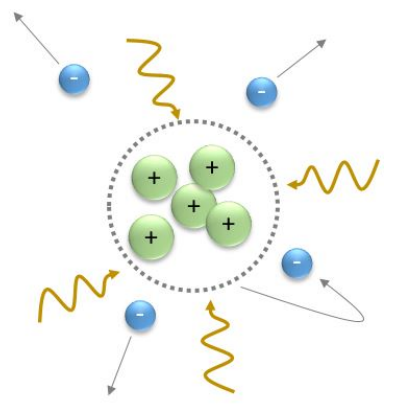

c.

FIGURE I.4: Transition from ion and electrons to plasma. Figure inspired from [Morozov, 2013].

A plasma can be created via different mechanisms. Thermal ionisation (particle collisions) is the most important one and can be found everywhere in the universe. When the matter is heated up to several thousands of degrees, molecules can split to form atoms. For temperatures above $10.000 \mathrm{~K}^{\circ}$, atoms themselves can split to electrons and positive ions. These temperature levels are reached in all stars, making their composition purely made of plasma, what explains the definition of fourth state of matter [Batani et al., 2018]. Plasma can be found almost everywhere around us such as candle flames or some light sources containing weakly ionised plasma discharges of rare gases (Ne, Xe etc.). Metal-cutting/welding using electrical arcs are also processes producing plasma. We are more interested in this thesis on laser-driven plasmas. Plasma result to be a very interesting medium to study for its ability to sustain strong electrical $(>\mathrm{GeV} / \mathrm{cm})$ and magnetic fields ( $>\mathrm{kT}$ ). Having a periodical response to external excitation (see plasma frequency later in this section) is also one of its important characteristics used to accelerate particles up to relativistic velocities. The possible mechanisms of creating a plasma using a laser are shown in this section. The following Fig.I.5, inspired from [Batani et al., 2018], shows a map identifying some of the plasma types found in nature or generated in laboratories. 


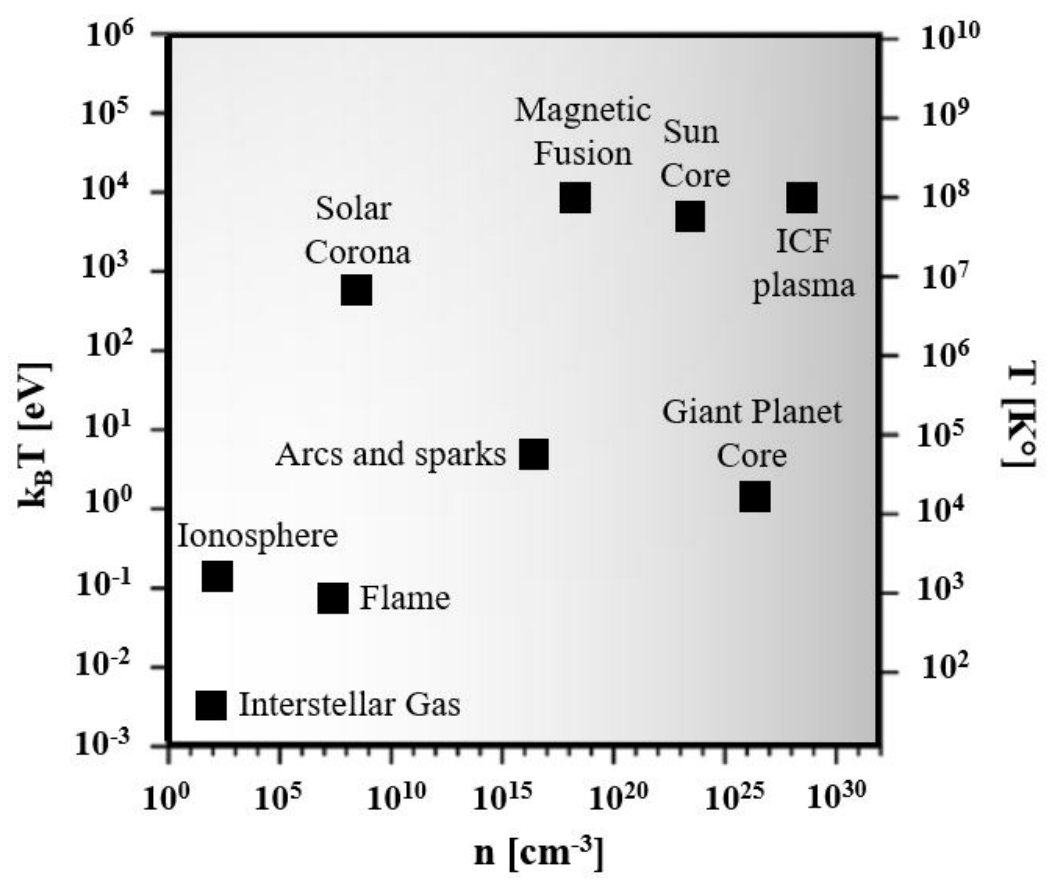

FIGURE I.5: Plasma examples that can be fund in nature or laboratories.

\section{I.2.2 Ionisation processes}

The development of high power lasers has opened the door of new research fields where matter is taken to extreme conditions [Protopapas et al., 1997]. The interaction between a focused high intensity beam and matter can ionize the latter and form a plasma via four mechanisms.

- Single photon ionisation: is the simplest case and consists of pulling off an electron from a stable state, inside the atomic potential, up to the continuum state via photo-electrical effect. The photon used to excite the electron should have an energy $E_{a}=h v_{a}$ higher than the electron binding energy (or ionization binding potential) $E_{B}$ where $E_{a} \geq E_{B}$ (see Fig.I.6.a).

- Multi-photon ionisation: occurs for intensities higher than $10^{10} \mathrm{~W} / \mathrm{cm}^{2}$, where a superposition of low energy photons $E_{b}$ (where $E_{b}=h v_{b}<E_{a}$ ) in time and space, can excite the electron up to virtual intermediate states and so, for $\sum h v_{b} \geq E_{B}$, an electron can be released out of the atomic potential (see Fig.I.6.b).

- Tunnel ionisation: happens when the laser's electric field is strong enough to modify the shape of the binding potential. The latter bends for each laser amplitude oscillation, reducing the electron potential barrier and "giving birth" to a finite possibility of electron tunnelling through the barrier and so, for the electron escape (see Fig.I.6.c).

- Over The Barrier Ionisation (OTBI): shown in Fig.I.6.d, occurs for high intensities $\left(>10^{14} \mathrm{~W} / \mathrm{cm}^{2}\right)$, where the laser's electric field is so high that it twists dramatically the binding potential of electron. The latter becomes even higher than the potential of laser electric field. Thus, the electron escapes from the high potential region to the lower one. The laser intensity $I_{a p p}$, or "appearance intensity" [Gibbon, 2005] needed for OTBI is given 
by

$$
I_{a p p}=\frac{c E_{B}^{4}}{128 \pi Z^{2} e^{6}} \simeq 4 \times 10^{9}\left(\frac{E_{B}}{\mathrm{eV}}\right)^{4} Z^{-2} \mathrm{Wcm}^{-2}
$$

Here, $Z$ is the charge of the created ion. For example, $I_{a p p}$ values needed to ionize a hydrogen atom to $\mathrm{H}^{+}$or a $\mathrm{He}$ atom to $\mathrm{He}^{+}$and $\mathrm{He}^{2+}$ are respectively $1.37 \times 10^{14}, 1.46 \times 10^{15}$ and $8.77 \times 10^{15} \mathrm{Wcm}^{-2}$. Values of $E_{B}$ can be found in [Sansonetti and Martin, 2005].

(a)

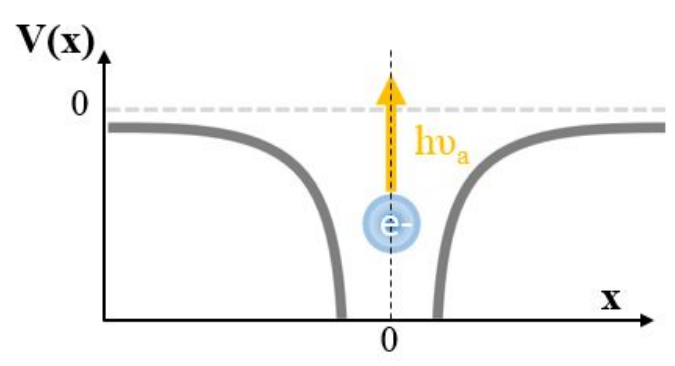

(c)

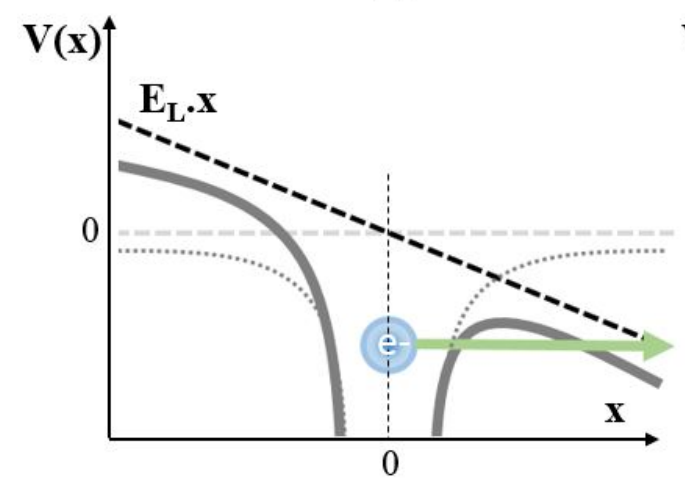

(b)

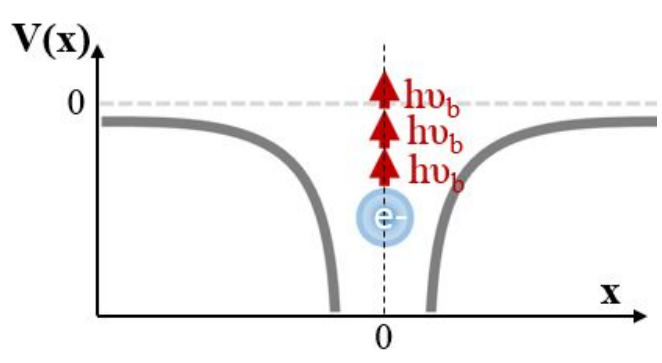

(d)

FIGURE I.6: Different ionization mechanisms of matter. a, b, c and d correspond respectively to single photon, multi-photon, tunnel and OTB ionization.

It is noteworthy to mention that in reality, only the ASE (Amplified Spontaneous Emission), also called pre-pulse, of high intensity lasers can have enough energy to create the plasma. Fig.I.7 is showing the temporal evolution of CLPU laser pulse where the laser contrast is higher than $10^{10}$. The interaction with the main laser pulse with matter is then described as a laser-plasma interaction rather than laser-matter one.

We can finally add that there are many laser applications where high contrast is needed. One of the most efficient techniques to remove the ASE from high intensity femtosecond laser pulses is performed via plasma mirrors [Thaury et al., 2007]. Its working principle is very straightforward. The ASE does not break the surface of the transparent substrate and is transmitted through. However, the higher intensity main pulse leading edge interacts with the plasma mirror (PM) creating a plasma on the PM. The generated plasma is dense enough (see more details on Sec.I.2.3.e) to reflect the main part of the main pulse leading to a contrast $10^{3}$ times higher [Rödel et al., 2011] than the initial contrast. 


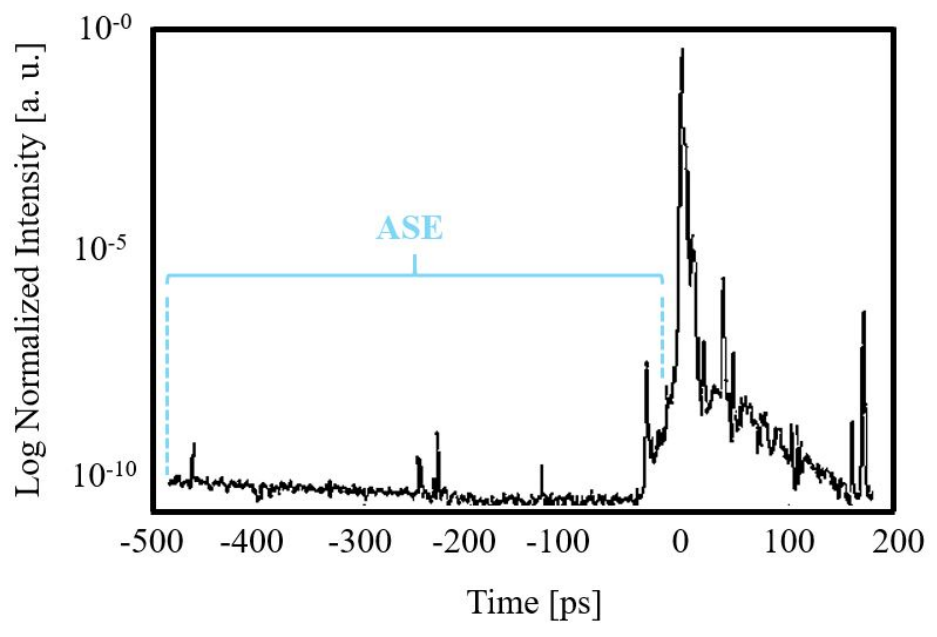

FIGURE I.7: Temporal evolution of an isolated VEGA 2 laser pulse delivered at CLPU laser facility. Courtesy from the head of CLPU laser division C.Méndez.

\section{I.2.3 Characteristic quantities of plasmas}

\section{I.2.3.a Plasma quasi-neutrality}

In plasmas, the forces acting between the charged particles are Coulomb electric ones characterized by a large range of interaction. The Coulomb potential generated by a particle of charge $q$ at distance $r$, in vacuum is given by

$$
\Phi(r)=\frac{q}{4 \pi \varepsilon_{0} r}
$$

All charged particles interact via the attractive and repulsive Coulomb forces. So, any local excess of charge (can be due to thermal electron movement, or to a charge separation) generates immediately strong current of charges and so, magnetic fields. As soon as the latter are generated, they act immediately on the plasma particles. Due to these multiple electrostatic interactions, the electron trajectory is affected by two main disturbances. The first, is a weak one, and is due to the action of distant charges. The second disturbance is important, and is due to local collisions which can be with an electron, atom or ion. Plasmas are sensitive to disturbances and can allow only small deviations from electrical neutrality. Consequently, these forces help to maintain a charge equilibrium, the electrical quasi-neutrality of the plasma which is expressed

$$
n_{e} q_{e}+\sum_{i} q_{i} n_{i}=0
$$

Where $n_{e}$ is the electronic density, $n_{i}$ is the ionic density and $q_{i}=Z n_{e}$ is the charge of the ions.

\section{I.2.3.b Landau length}

Landau length, $r_{0}$, represents the minimum approach distance between two electrons in a plasma. If we consider a scheme, where electrons and ions are evenly distributed in a plasma (one electron 
per cubic cell of dimension $d_{e}$, see Fig.I.8), we can write:

$$
d_{e, i}=n_{e, i}^{-1 / 3}
$$

Where $n_{e, i}$ refers to the electron or ion density. Considering $r_{0}$ the distance at which the potential interaction energy is equal to the mean kinetic energy of the electron and so we can write

$$
\frac{1}{2} m_{e} \mathrm{v}_{e}^{2}=k_{B} T_{e}=\frac{e^{2}}{4 \pi \varepsilon_{0} r_{0}}
$$

Here $\mathrm{v}_{e}$ is the most probable electron speed, $k_{B}$ is the Bolzmann constant $\left(1.38 \times 10^{23} \mathrm{~J} / \mathrm{K}\right), e$ is the elementary electron charge $\left(1.6 \times 10^{-19} \mathrm{C}\right), \varepsilon_{0}$ the vacuum permittivity $\left(1 / 36 \pi \times 10^{-9} \mathrm{~F} / \mathrm{m}\right)$. Thus, Landau length is written

$$
r_{0}=\frac{e^{2}}{4 \pi \varepsilon_{0} k_{B} T_{e}}
$$

Knowing $r_{0}$ and $d_{e}$, we can define the plasma parameter $g_{p}$, aiming to distinguish between two types of plasma, where $g_{p}=r_{0} / d_{e}$, so

$$
g_{p}=\frac{e^{2} n_{e}^{1 / 3}}{4 \pi \varepsilon_{0} k_{B} T_{e}}, \quad g_{p} \ll 1
$$

For $d_{e} \gg r_{0}$, the plasma is described as "standard kinetic plasma" where the kinetic energy is much higher than the potential energy. The second case, where $d_{e} \geq r_{0}$, corresponds to the inverse situation, where we have a "strongly correlated plasma". For a plasma of electron temperature $T_{e}=2 \mathrm{keV}$, Landau length is equal to $\simeq 7.1937 \times 10^{-13} \mathrm{~m}$.

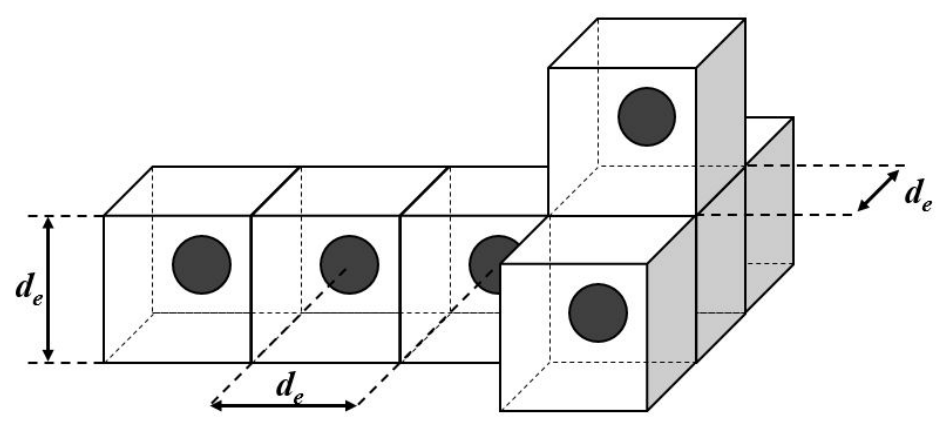

FIGURE I.8: Scheme for particle spatial distribution in cells.

\section{I.2.3.c Electronic density distribution and Debye length}

The phenomena of charge screening in an ionised environment is due to the Debye-Hückel effect. The latter was discovered by the two physicists Peter Debye and Erich Hückel in 1923. Let us consider a plasma of uniform temperature, $T_{e}=$ const. Due to the Coulomb force of the ion, the electron density is not constant. The elementary volume $d V$, shown in Fig.I.9, is then subjected to the electrical force $F_{E}$, such as 


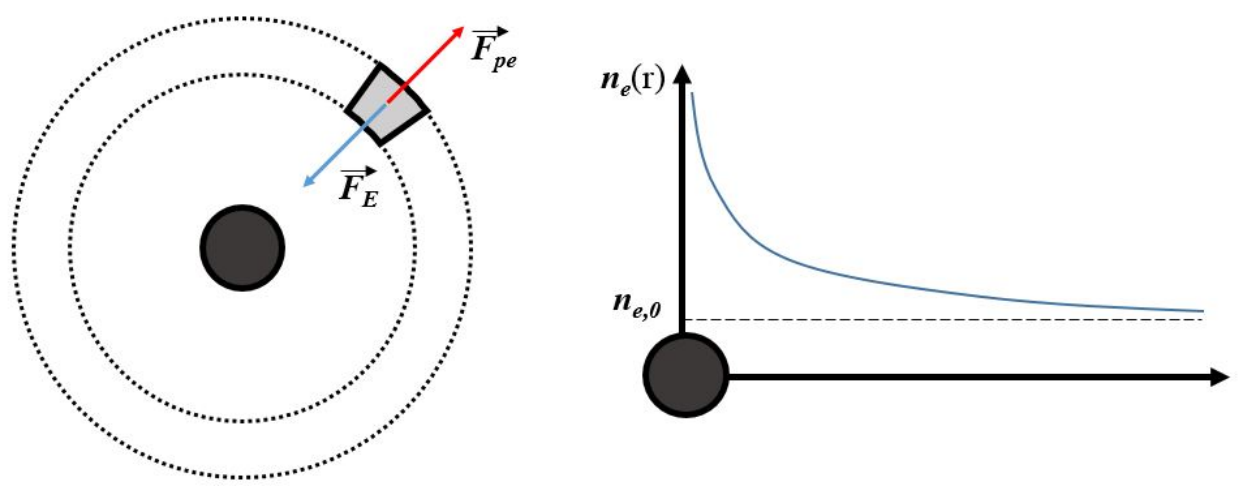

FIGURE I.9: Forces applied to the electrons is an element of volume $d V$ (Right). Electron density distribution in function of distance relative to the ion position.

$$
\boldsymbol{F}_{E}=n_{e} e \boldsymbol{E}=-n_{e} e \nabla \Phi(r)
$$

The element of volume $d V$ is also subjected to the pressure force resulting from the electronic density gradient near to the ion due to electron accumulation.

$$
F_{p e}=-\nabla n_{e} k_{B} T_{e}=-k_{B} T_{e} \nabla n_{e}
$$

Since the forces $\boldsymbol{F}_{E}$ and $\boldsymbol{F}_{p e}$ are of opposite sign, in equilibrium we get

$$
\boldsymbol{F}_{p e}+\boldsymbol{F}_{E}=0
$$

So

$$
-n_{e} e \nabla \Phi(r)-k_{B} T_{e} \nabla n_{e}=0
$$

For spherical symmetry reasons (due to purely radial forces), we can write

$$
\frac{1}{n_{e}} \frac{d n_{e}}{d r}=-\frac{e}{k_{B} T_{e}} \frac{d \Phi}{d r}
$$

Integrating Eq.I.20, gives the Boltzmann distribution of electrons in the electrostatic potential

$$
n_{e}=n_{e, 0} \exp \left(\frac{-e \Phi(r)}{k_{B} T_{E}}\right)
$$

Considering that the kinetic energy of electron is higher than the potential energy $e \Phi$, we can expand Eq.I.21 in Taylor series

$$
n_{e} \simeq n_{e, 0}\left(\frac{-e \Phi(r)}{k_{B} T_{E}}\right)
$$

We can then evaluate the ion potential $\phi$ by using Maxwell-Poisson equation, so

$$
\nabla . E=\frac{\rho}{\varepsilon_{0}}=\frac{-n_{e} e+n_{i, 0} q_{i}}{\varepsilon_{0}}=\frac{-n_{e}+n_{e, 0}}{\varepsilon_{0}} e=-\Delta \Phi(r)
$$


We replace Eq.I.22 into Eq.I.23 we find

$$
\Delta \Phi(r)=\frac{n_{e, 0} e^{2}}{\varepsilon_{0} k_{B} T_{e}} \Phi(r)=\frac{\Phi(r)}{\lambda_{D e}^{2}}
$$

Where $\lambda_{D e}$ is the Debye screening length and is written

$$
\lambda_{D e}=\sqrt{\frac{\varepsilon_{0} k_{B} T_{e}}{n_{e} e^{2}}}
$$

The Laplacian in the spherical coordinates is expressed as $\Delta(r)=r^{-2}(\partial / \partial r)\left(r^{2} \partial / \partial r\right)$. Integrating Eq.I.24 gives the following Debye potential expression of a charge $\mathrm{q}$

$$
\Phi(r)=\frac{q}{4 \pi \varepsilon_{0} r} \exp \left(-\frac{r}{\lambda_{D e}}\right)
$$

We can conclude that in a plasma, the Debye potential replaces the Coulomb potential (of an isolated charge). Also, the $\lambda_{D e}$ is considered as the characteristic scale of the decrease in potential, associated to the effect of screening. The sphere around the ion of a radius $\lambda_{D e}$ is called the Debye sphere. The number of electron $N_{D e}$ inside the Debye sphere is

$$
N_{D e}=\frac{4}{3} \pi n_{e} \lambda_{D e}^{3}
$$

It is worth mentioning that the screening effect is negligible if $N_{D e} \ll 1$. For $N_{D e} \gg 1$, the screening effect is strong and so, the electron behaves almost as a gas in relation to the ions.

\section{I.2.3.d Plasma oscillation: electronic plasma frequency}

We consider a neutral plasma section of length $\mathrm{L}$, formed by stationary ions and electrons, where $n_{e}=n_{i}$. We can imagine a situation where, due to an external perturbation in the $x$ direction, the electrons are puled off to one direction. Consequently, in one side, there will be more electrons than ions, meanwhile in the other side, there will be the contrary. This situation is equivalent to a charged parallel-plate capacitor system (see Fig.I.10). This charge separation will generate an electric field $E$ given by

$$
E=-\frac{\sigma_{s}}{\varepsilon_{0}}
$$

Where $\sigma_{s}$ is the surface charge density $\left(\sigma_{s}=-n_{e} e x\right)$. A restoring force $F=-e E$ will take place, and the equation for the electrons movement would be written

$$
\frac{d^{2} x}{d t^{2}}=-\frac{n_{e} e^{2}}{m_{e} \varepsilon_{0}} x=-\omega_{p e}^{2} x
$$

This expression is similar to an oscillator equation of specific angular frequency $\omega_{p e}$, called the electronic plasma frequency given by

$$
\omega_{p e}=\sqrt{\frac{n_{e} e^{2}}{m_{e} \varepsilon_{0}}}
$$


It is noteworthy to add that the plasma wavelength $\lambda_{p}=2 \pi c / \omega_{p e}$ (or plasma period) and the associated plasma wavenumber $k_{p}=2 \pi / \lambda_{p}$ are two important plasma quantities related to the plasma frequency. It is good to mention that $\lambda_{p}$ is equal to

$$
\lambda_{p}[\mu \mathrm{m}] \simeq \frac{3.3 \times 10^{10}}{\sqrt{n_{e}\left[\mathrm{~cm}^{-3}\right]}}
$$

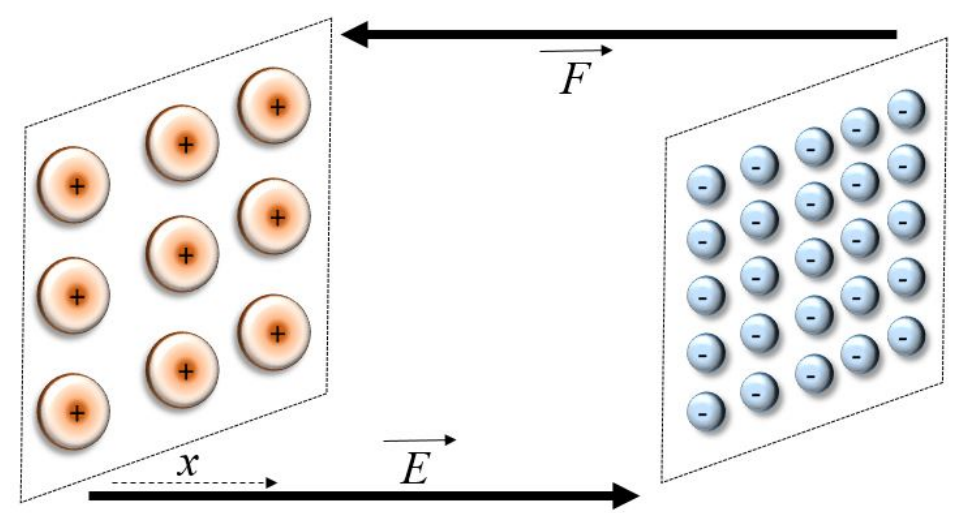

FIGURE I.10: Charge separation in a plasma oscillation.

\section{I.2.3.e Critical density}

The notion of critical density is very important when we consider the propagation of an electromagnetic wave in a plasma. The critical density $n_{c}$ refers to the value of density above which an electromagnetic wave cannot propagate in a plasma. We can obtain the expression of $n_{c}$ considering an incident planar electro magnetic wave characterized by $\boldsymbol{E}$ and $\boldsymbol{B}$, the electric and magnetic fields given by

$$
\begin{aligned}
& \boldsymbol{E}=\operatorname{Re}\left(\boldsymbol{E}_{0}\right) e^{i\left(\mathbf{k} \cdot \mathbf{r}-\omega_{L} t\right)} \\
& \boldsymbol{B}=\operatorname{Re}\left(\boldsymbol{B}_{0}\right) e^{i\left(\mathbf{k} \cdot \mathbf{r}-\omega_{L} t\right)}
\end{aligned}
$$

Where $k$ is the wave number and $\omega_{L}$ the laser angular frequency (pulsation). Transverse polarization is one of the important characteristics of electromagnetic waves and so k. $E=0$. Maxwell equations represent the evolution of both the electric field and magnetic field and are written as

$$
\begin{aligned}
& \nabla . E=\frac{\rho}{\varepsilon_{0}} \\
& \nabla . B=0 \\
& \nabla \times E=\frac{\partial B}{\partial t} \\
& \nabla \times B=\mu_{0} \mathbf{j}+\mu_{0} \varepsilon_{0} \frac{\partial E}{\partial t}
\end{aligned}
$$


Where $\mathbf{j}=-n_{e} e \mathbf{v}_{\mathbf{e}}$ is the density current vector and $\mu_{0}$ is the vacuum permeability and is equal to $4 \pi \times 10^{-7} \mathrm{H} / \mathrm{m}$. We can rewrite these expressions in Fourier space where they become

$$
\begin{aligned}
& i \mathbf{k} . E=\frac{\rho}{\varepsilon_{0}} \\
& i \mathbf{k} \cdot \boldsymbol{B}=0 \\
& \mathbf{k} \times E=\omega_{L} B \\
& \mathbf{k} \times \boldsymbol{B}=-i \mu_{0} \mathbf{j}-\mu_{0} \varepsilon_{0} \omega_{L} E
\end{aligned}
$$

We note that we can replace $\mu_{0} \varepsilon_{0}$ with $1 / c^{2}$.

From the electron's movement equation in the Fourier space, one can write the expression of $\mathbf{v}_{\mathbf{e}}$ by neglecting the magnetic field effects $\left(\mathrm{v}_{e} / c \ll 1\right)$

$$
m_{e} \frac{d \mathbf{v}_{e}}{d t}=-e \mathbf{E}
$$

and so $\mathbf{v}_{\mathbf{e}}$ is written

$$
\mathbf{v}_{\mathbf{e}}=\frac{-e}{m_{e}} \int \boldsymbol{E} d t=\frac{-i e}{\omega_{L} m_{e}} \boldsymbol{E}
$$

We replace both, the current density and the magnetic field expressions in the Maxwell-Faraday equation and we get

$$
\mathbf{k} \times \boldsymbol{B}=\mathbf{k} \times\left(\frac{\mathbf{k} \times \boldsymbol{E}}{\omega_{L}}\right)=\frac{\omega_{p e}^{2}}{c^{2} \omega_{L}} \boldsymbol{E}-\frac{\omega_{L}}{c^{2}} \boldsymbol{E}
$$

Considering a planar electromagnetic wave where $\mathbf{k} \cdot E=0$ and by using the vectorial identity $\mathbf{k} \times \mathbf{k} \times \boldsymbol{E}=\mathbf{k}$. $(\mathbf{k} . \boldsymbol{E})-\mathrm{k}^{2} \boldsymbol{E}$, the equation of propagation for the electric field can be written

$$
\left(\omega_{L}^{2}-\omega_{p e}^{2}-\mathrm{k}^{2} c^{2}\right) E=0
$$

The dispersion relation of the electromagnetic wave is then given by

$$
\mathrm{k}^{2} c^{2}=\omega_{L}^{2}-\omega_{p e}^{2}
$$

From Eq.I.45, we can see clearly that only electromagnetic waves of $\omega_{L}>\omega_{p e}$ can propagate in the plasma. This condition is satisfied only if, the wavevector $\mathrm{k}$ is real. In case the solution of Eq.I.45 is complex, the laser pulse equation is then equivalent to an evanescence wave and no propagation is permitted (where the laser gets reflected). Thus, the critical density is given by the condition $\omega_{p e}=\omega_{L}$ and is written

$$
n_{c}=\frac{\varepsilon_{0} m_{e}}{e^{2}} \omega_{L}^{2} \simeq 1.117 \times 10^{21} \lambda_{\mu \mathrm{m}}^{-2} \mathrm{~cm}^{-3}
$$

Where $\lambda_{\mu \mathrm{m}}$ corresponds to the laser wavelength $\lambda_{L}$ in units of microns. The critical density $n_{c}$ is a very relevant parameter separating two different regimes for electron acceleration corresponding to two ranges of densities, $n_{c}<n_{e}$ and $n_{c}>n_{e}$.

From one side is a regime in which the mechanism of acceleration is due to laser propagation in the under-dense matter (gas targets) presented later in this thesis in Laser Wakefield Acceleration section. On the other side is a regime in which the laser energy is partially transmitted to electrons in dense matter (using solid targets) in which the laser penetration is limited, presented 
later in this thesis as Target Normal Sheath Acceleration [Roth and Schollmeier, 2016]. Fig.I.11 shows how $n_{c}$ is separating between these two regimes of electron acceleration together with the corresponding type of targetry systems.

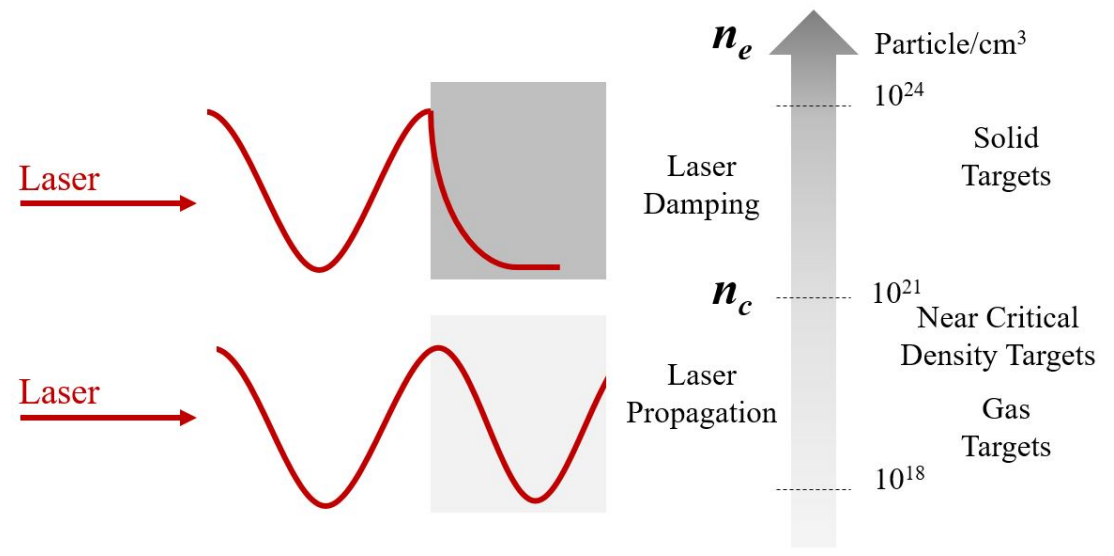

FIGURE I.11: Role of $n_{c}$ in separating two regimes of laser-matter interaction.

We note there are some ways to overcome this limit. For example, according to [Kruer, 1988, Gibbon, 2005], placing the target at an angle $\theta$ from target normal is one possibility. Considering (see Fig.I.12) a p-polarised light, the parallel component of the electric field will be reflected at $n_{e}=n_{c} \cos ^{2}(\theta)$ while the transverse component will tunnel up to the critical density $n_{c}$. This technique is commonly used to increase laser penetration in solid targets so that more electrons can be accelerated. Another technique is also used to increase laser penetration where it consists of doubling the laser frequency using non-linear methods (since the critical density is inversely proportional to the laser wavelength). Finally, using high intensity lasers can cause an increase of the critical density (considering the relativistic case where there is a relativistic increase in the electron mass) where $n_{c}^{\prime}=\gamma n_{c}$, where $\gamma$ is the relativistic Lorentz factor, and so, the laser can penetrate deeper inside the plasma.

\section{I.2.4 Motion of an electron in a laser field}

Studying the interaction between a laser pulse and a plasma, starts by understanding the motion of a single free electron inside a laser field. Thus, let us consider an electromagnetic wave (laser pulse) of electromagnetic fields $E$ and $B$ incident on an isolated free electron at rest in the laboratory frame. The motion of the electron will be governed by the following equations

$$
\begin{aligned}
& \text { Lorentz equation } \Longrightarrow \quad \frac{d \mathbf{p}}{d t}=-e\left(\boldsymbol{E}+\mathbf{v}_{\mathbf{e}} \times \boldsymbol{B}\right) \\
& \text { Energy equation } \Longrightarrow \quad \frac{d}{d t}\left(\gamma m c^{2}\right)=-e\left(\mathbf{v}_{\mathbf{e}} \cdot \boldsymbol{E}\right)
\end{aligned}
$$

Where $\mathbf{v}_{\mathbf{e}}$ is the electron velocity and $\mathbf{p}=\gamma m \mathbf{v}_{\mathbf{e}}$, is the electron momentum depending on $\gamma$, the relativistic Lorentz factor where

$$
\gamma=\sqrt{1+\left(p / m_{e} c\right)^{2}}=\frac{1}{\sqrt{1-\left(\frac{\mathrm{v}_{e}}{c}\right)^{2}}}
$$




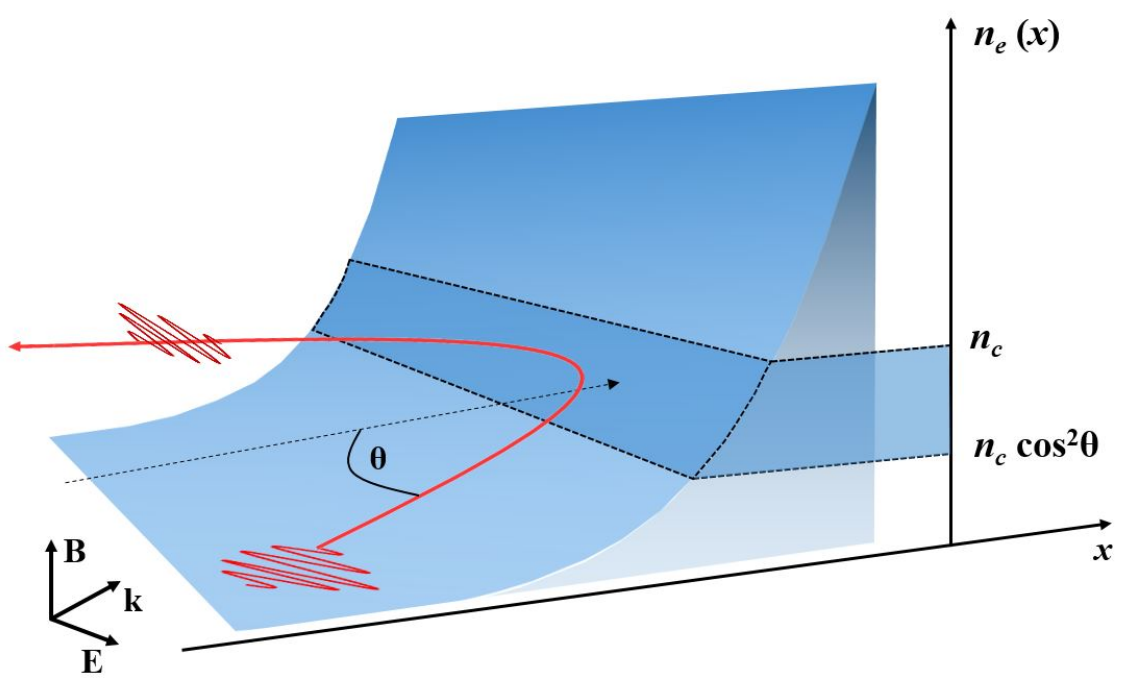

FIGURE I.12: Simplified scheme showing both, beam penetration and reflection of a p-polarized laser pulse incident onto a gradient of density surface. This figure has been inspired from [Gibbon, 2005]

In the non-relativistic case, where the electron velocity is much lower than the speed of light $\left(\mathrm{v}_{e} \ll c\right.$ and $\gamma \approx 1$ ), the magnetic component of the Lorentz equation (Eq.I.47) can be neglected. Thus, the electron moves at the velocity $\mathbf{v}_{\mathbf{q}}$, called "quiver velocity" and so we can write

$$
m_{e} \frac{d \mathbf{v}_{\mathbf{q}}}{d t}=-e \boldsymbol{E}
$$

Both, the electric field $E$ and magnetic field $B$ can be written as a function of the vector potential A.

$$
\begin{aligned}
& \boldsymbol{E}=-\frac{\partial A}{\partial t} \\
& \boldsymbol{B}=\nabla \times A
\end{aligned}
$$

We consider an incident electromagnetic wave, transversally polarized (along the y axis) and propagating along the x axis. The potential vector of this wave is given by $A=A_{\perp}=A \cos (\varphi) \mathbf{e}_{\mathbf{y}}$, where $\varphi=k x-\omega_{L} t$ is the phase, $k=2 \pi / \lambda_{L}=\omega_{L} / c$ is the wave vector. The transverse component of the electron impulsion is then written

$$
\mathbf{p}_{\perp}=e A_{\perp}
$$

According to [Gibbon, 2005], the components of the electron momentum in the laboratory frame (for a linear polarization) are given by

$$
\begin{aligned}
& \mathrm{p}_{x}=\frac{a_{0}^{2}}{4}(1+\cos (2 \varphi)) \\
& \mathrm{p}_{y}=a_{0} \cos (\varphi)
\end{aligned}
$$


These expressions can be integrated to find the trajectory equations given by

$$
\begin{aligned}
& x=\frac{a_{0}^{2}}{4}\left(\varphi+\frac{1}{2} \sin (2 \varphi)\right) \\
& y=a_{0} \sin (\varphi)
\end{aligned}
$$

We have introduced the peak value $a_{0}$ of the normalized vector potential $\mathbf{a}=e A / m_{e} c$, which provides also the electron maximum oscillation velocity $\mathrm{v}_{o s} \equiv \mathrm{v}_{\perp}$. The so called laser strength parameter $a_{0}$ is then expressed

$$
a_{0}=\frac{e A_{0}}{m_{e} c}=\frac{e E}{m_{e} c \omega_{L}}=0.85 \times \sqrt{I_{18} \lambda_{L(\mu \mathrm{m})}^{2}}
$$

Here $I_{18}$ is the laser intensity in units of $10^{18} \mathrm{~W} / \mathrm{cm}^{2}$ (with $I=\varepsilon_{0} c E^{2} / 2$ ), and $\lambda_{L(\mu \mathrm{m})}$ is the laser wavelength in unit of microns. According to Eq.I.56 and Eq.I.57, one can clearly see the differences between the electron motion in the transverse and in the longitudinal directions. In the transverse one, we can observe how the electron oscillates with the laser frequency. In the longitudinal direction, apart from the fact that the electron oscillates with a frequency equal to two times the laser frequency, it also acquires a net positive drift in the laser propagation axis. The trajectories described by the electron orbits in the laboratory frame are shown in Fig.I.13 for $a_{0}$ values of $0.5,1$ and 4 , for a Ti:Sapphire laser $\left(\lambda_{L}=0.8 \mu \mathrm{m}\right)$ corresponding respectively to the laser intensities $5.40 \times 10^{17}, 2.16 \times 10^{18}$ and $3.46 \times 10^{19} \mathrm{Wcm}^{2}$.

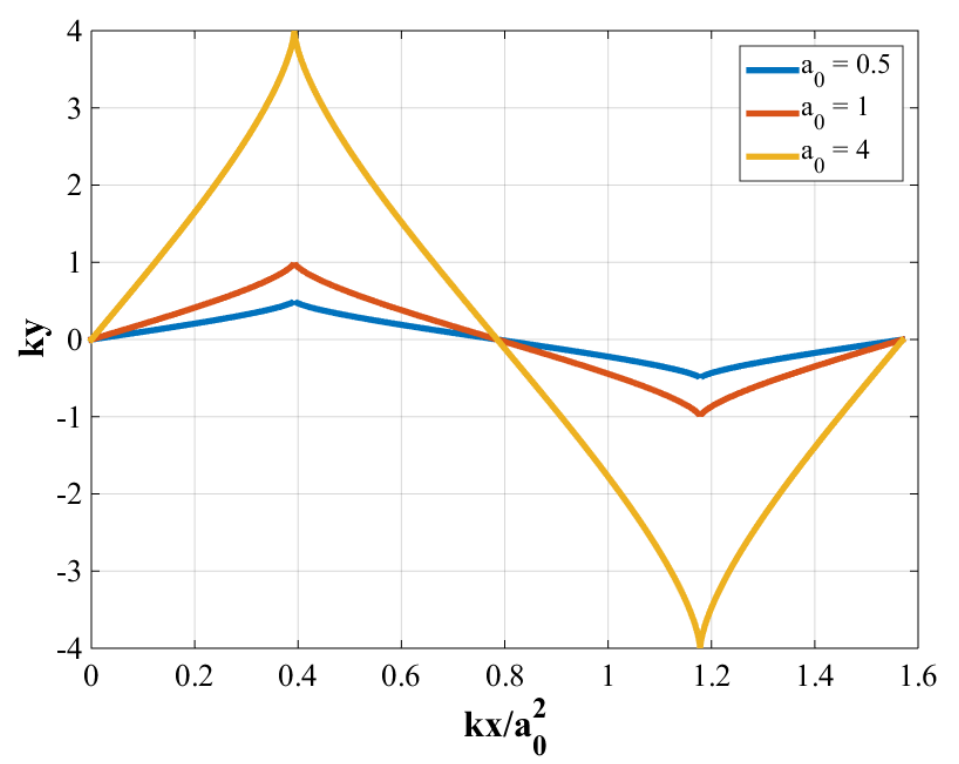

FIGURE I.13: Electron orbits in the laboratory frame for different normalized vector potential peak values $a_{0}=0.5,1$ and 4 .

The amplitude of the motion is proportional to $a_{0}$ in the transverse direction but to $a_{0}^{2}$ in the longitudinal one. Thus, the electron starts drifting in the laser propagation axis with an average momentum $\mathrm{p}_{D} \equiv \overline{\mathrm{p}_{x}}=a_{0}^{2} / 4$. Here $D$ stands for "drift". This drift corresponds to a drift velocity 
$\mathrm{v}_{D}$ given by [Gibbon, 2005] as

$$
\mathrm{v}_{D}=c \frac{a_{0}^{2}}{4+a_{0}^{2}}
$$

Up to now, we have considered the laboratory frame as a reference. Let us consider now, the frame co-propagating with the average velocity of the electron. According to [Gibbon, 2005], the electron trajectory is given by

$$
\begin{aligned}
& x=\frac{1}{2} q^{2} \sin (2 \varphi) \\
& y=2 q \sin (\varphi)
\end{aligned}
$$

where $q=a_{0} /\left(2\left(1+a_{0}^{2} / 2\right)^{0.5}\right)$. Fig.I.14 highlights the evolution of electron amplitude oscillation as a function of laser intensity. This figure is known as the "figure-of-eight".

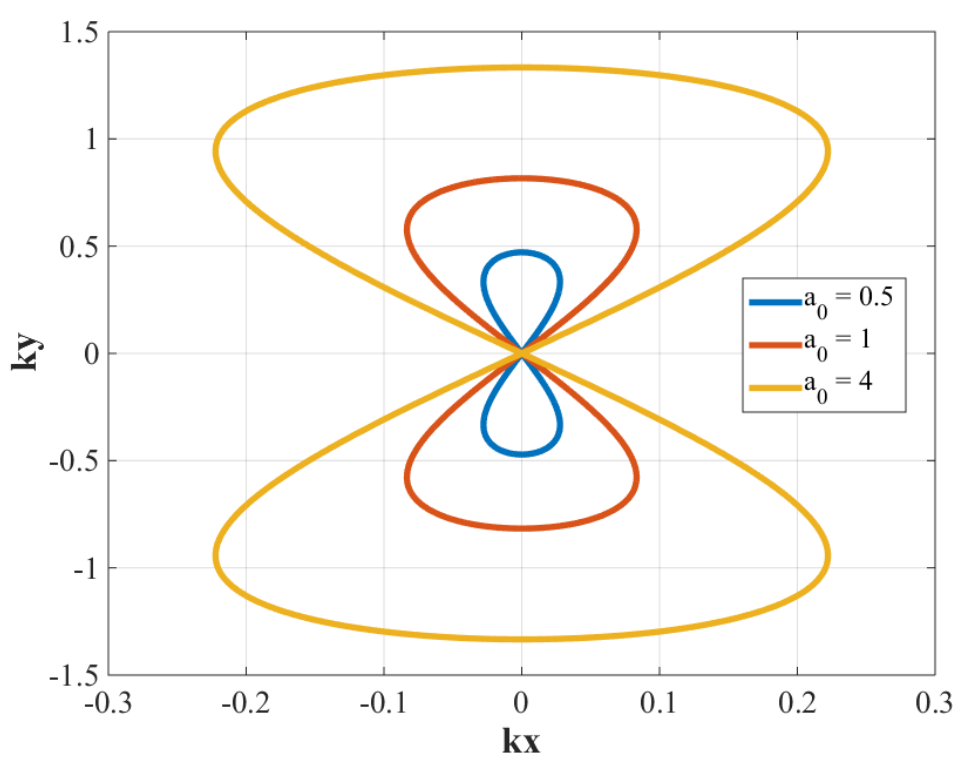

FIGURE I.14: Electron orbits in the rest frame for different normalized vector potential peak values $a_{0}=0.5,1$ and 4 .

\section{I.2.5 The ponderomotive force}

There exist many laser-based mechanisms to accelerate electrons. For laser intensities on the order of $10^{16} \mathrm{~W} . \mathrm{cm}^{-2}$, collisional and resonant absorption are considered as the main mechanisms responsible for electron acceleration. At higher laser intensities, such as $10^{18} \mathrm{Wcm}^{-2}$ and more, collective effects becomes dominant (the magnetic term of the Lorentz force becomes no more negligible) and the energy transfer from laser pulse to electrons is carried out by means of the so called 'ponderomotive force'. It has been demonstrated according to Eq.I.50, that in the linear regime (non-relativistic), the electron moves at quiver velocity $\mathbf{v}_{q}$, if the latter is placed inside an electromagnetic field. Let us consider, for example, a case (non-relativistic one) at which the 
laser is considered only as a perturbation. Considering the cold plasma approximation, we can expand the following variables to zero, first and second orders as

$$
\begin{aligned}
\boldsymbol{E} & =\boldsymbol{E}^{(0)}+\delta \boldsymbol{E}^{(1)}+\delta \boldsymbol{E}^{(2)} \\
\boldsymbol{B} & =\boldsymbol{B}^{(0)}+\delta \boldsymbol{B}^{(1)}+\delta \boldsymbol{B}^{(2)} \\
\mathbf{v}_{e} & =\mathbf{v}_{e}^{(0)}+\delta \mathbf{v}_{e}^{(1)}+\delta \mathbf{v}_{e}^{(2)}
\end{aligned}
$$

The cold plasma is at equilibrium so, $\boldsymbol{E}^{(0)}, \boldsymbol{B}^{(0)}$ and $\mathbf{v}_{e}^{(0)}$ are equal to zero. Considered as a first order perturbation, $\delta \boldsymbol{E}^{(1)}$ and $\delta \boldsymbol{B}^{(1)}$ can be replaced by $\boldsymbol{E}_{L}$ and $\boldsymbol{B}_{L}$, the laser electric and magnetic fields respectively. Using the identity $d / d t=\partial / \partial t+\mathbf{v} . \nabla$ (where $\nabla=\partial / \partial \mathbf{r}$ ), we can write then the Lorentz equation:

$$
m_{e} \frac{d \mathbf{v}_{e}}{d t}=-e\left(\boldsymbol{E}+\mathbf{v}_{e} \times \boldsymbol{B}\right)
$$

To get the $1^{\text {st }}$ order response of the plasma electrons, we replace Eq.I.64 in to Eq.I.65

$$
m_{e} \frac{d \mathbf{v}_{e}}{d t}=m_{e}\left[\frac{\partial \mathbf{v}_{e}}{\partial t}+\mathbf{v}_{e} \cdot \nabla \mathbf{v}_{e}\right]=m_{e}\left[\frac{\partial}{\partial t}\left(\delta \mathbf{v}_{e}^{(1)}\right)\right]
$$

Lorentz equation is then written

$$
m_{e} \frac{\partial}{\partial t}\left(\delta \mathbf{v}_{e}^{(1)}\right)=-e \boldsymbol{E}_{L}
$$

The $2^{\text {nd }}$ order response of the electrons of the plasma is given by

$$
m_{e}\left[\frac{\partial}{\partial t}\left(\delta \mathbf{v}_{e}^{(2)}\right)+\left(\delta \mathbf{v}_{e}^{(1)} \cdot \frac{\partial}{\partial \mathbf{r}}\right)\left(\delta \mathbf{v}_{e}^{(1)}\right)\right]=-e \frac{\delta \mathbf{v}_{e}^{(1)}}{c} \times B_{L}
$$

By applying the curl operation $(\partial / \partial \mathbf{r})$ to Eq.I.67, and according to Eq.I.51 and Eq.I.52 we can get

$$
-\left(\frac{m_{e}}{e}\right)\left(\frac{\partial}{\partial t}\right)\left[\left(\frac{\partial}{\partial \mathbf{r}}\right) \times \delta \mathbf{v}_{e}^{(1)}\right]=\left(\frac{\partial}{\partial \mathbf{r}}\right) \times E_{L}=-\frac{1}{c}\left(\frac{\partial}{\partial t}\right) B_{L}
$$

And so, $\boldsymbol{B}_{L}$ can be written as

$$
\boldsymbol{B}_{L}=-\left(\frac{m_{e} c}{e}\right)\left(\frac{\partial}{\partial \mathbf{r}}\right) \delta \mathbf{v}_{e}^{(1)}
$$

We can then write

$$
\begin{aligned}
m_{e} \frac{\partial}{\partial t}\left(\delta \mathbf{v}_{e}^{(2)}\right) & =-m_{e}\left(\delta \mathbf{v}_{e}^{(1)} \cdot \frac{\partial}{\partial \mathbf{r}}\right)\left(\delta \mathbf{v}_{e}^{(1)}\right)-m_{e} \delta \mathbf{v}_{e}^{(1)} \times\left(\frac{\partial}{\partial \mathbf{r}} \times \delta \mathbf{v}_{e}^{(1)}\right) \\
& =-\frac{\partial}{\partial \mathbf{r}}\left(\frac{1}{2} m_{e} \delta \mathbf{v}_{e}^{(1)^{2}}\right)
\end{aligned}
$$

The ponderomotive force is the average of the quantity expressed in Eq.I.71 over a laser period. Let us consider a monochromatic plane wave linearly polarized and propagating in the $\mathrm{x}$ direction 
with an electric field $\boldsymbol{E}_{L}=\boldsymbol{E}_{0}(r, t) \sin \left(\omega_{L} t+k x\right) \mathbf{e}_{y}$, where $E_{0}$ is the slowly varying envelope of the electric field. The mean non relativistic ponderomotive force over a laser period is written

$$
\begin{aligned}
\mathbf{F}_{n r-\text { Pond }}=-\frac{\partial}{\partial \mathbf{r}}\left\langle\frac{1}{2} m_{e} \delta \mathbf{v}_{e}^{(1)^{2}}\right\rangle=-\frac{\partial}{\partial \mathbf{r}}\left\langle\frac{e^{2} \boldsymbol{E}^{2}}{2 m_{e} \omega_{L}^{2}}\right\rangle=-m_{e} c^{2} \frac{\partial}{\partial \mathbf{r}}\left\langle\frac{a_{0}^{2}}{2}\right\rangle & \\
& =-\frac{e^{2}}{2 m_{e} \omega_{L}^{2} c \varepsilon_{0}} \nabla \boldsymbol{I}(\mathbf{r})
\end{aligned}
$$

Eq.I.72 is not valid any more for the relativistic case. The motion of the electron described by the Lorentz equation needs corrections and is written

$$
\frac{\partial \mathbf{p}_{e}}{\partial t}+\left(\mathbf{v}_{e} \cdot \frac{\partial}{\partial \mathbf{r}}\right) \mathbf{p}_{e}=-e\left(\boldsymbol{E}_{L}+\frac{\mathbf{v}_{e}}{c} \times \boldsymbol{B}_{L}\right)
$$

With

$$
\left(\gamma_{e}-1\right) m_{e} c^{2}=\sqrt{m_{e}^{2} c^{4}+\mathbf{p}_{e}^{2} c^{2}}-m_{e} c^{2}
$$

Where $\mathbf{p}_{e}=\gamma_{e} m_{e} \mathbf{v}_{e}$ is the mean electron momentum, $\gamma_{e}$ is the mean Lorentz factor and $\mathbf{v}_{e}$ is the mean electron velocity. According to [Huba, 2013], using the following vectorial identities

$$
\begin{aligned}
\nabla(A \cdot B) & =A \times(\nabla \times B)+B \times(\nabla \times A)+(A \cdot \nabla) B+(B \cdot \nabla) A \\
A \times(\nabla \times B) & =(\nabla B) \cdot A-(A \cdot \nabla) B
\end{aligned}
$$

And if we notice that

$$
\begin{aligned}
2 \gamma_{e}\left(m_{e} c\right)^{2}\left(\frac{\partial}{\partial \mathbf{r}}\right)\left(\gamma_{e}-1\right)=\left(m_{e} c\right)^{2}\left(\frac{\partial}{\partial \mathbf{r}}\right)\left(\gamma_{e}\right)^{2} & =\left(\frac{\partial}{\partial \mathbf{r}}\right)\left(\mathbf{p}_{e} \cdot \mathbf{p}_{e}\right) \\
& =2\left[\mathbf{p}_{e} \cdot\left(\frac{\partial}{\partial \mathbf{r}}\right)\right]\left(\mathbf{p}_{e}\right)+2 \mathbf{p}_{e} \times\left[\left(\frac{\partial}{\partial \mathbf{r}}\right) \times \mathbf{p}\right]
\end{aligned}
$$

and

$$
\left[\mathbf{v}_{e} \cdot\left(\frac{\partial}{\partial \mathbf{r}}\right)\right] \mathbf{p}_{e}=\left(\frac{\partial}{\partial \mathbf{r}}\right)\left(\gamma_{e}-1\right) m_{e} c^{2}-\mathbf{v}_{e} \times\left[\left(\frac{\partial}{\partial \mathbf{r}}\right) \times \mathbf{p}_{e}\right]
$$

Knowing that $\boldsymbol{E}=-(\partial \mathbf{A} / \partial t)-\nabla \Phi$ and $\boldsymbol{B}=c \nabla \times \mathbf{A}$, where $\mathbf{A}$ and $\Phi$ are the laser vector potential and the electrostatic potential respectively. We can then rewrite Eq.I.73 as

$$
\frac{\partial}{\partial t}\left(\mathbf{p}_{e}-e \mathbf{A}\right)=-\frac{\partial}{\partial \mathbf{r}}\left(\gamma_{e}-1\right) m_{e} c^{2}+\frac{\partial}{\partial \mathbf{r}}(e \Phi)+\mathbf{v}_{e} \times \underbrace{\left[\frac{\partial}{\partial \mathbf{r}} \times\left(\mathbf{p}_{e}-e \mathbf{A}\right)\right]}_{(\mathbf{1})}
$$

Supposing that the electrons are initially at rest, (1) is then equal to zero. Thus Eq.I.73 is given by

$$
\frac{\partial}{\partial t}\left(\mathbf{p}_{e}-e \mathbf{A}\right)=\mathbf{F}+\frac{\partial}{\partial \mathbf{r}}(e \Phi)
$$


so the non averaged (NA) relativistic ponderomotive force is written

$$
\mathbf{F}_{N A-r-P o n d}=-\frac{\partial}{\partial \mathbf{r}}\left(\gamma_{e}-1\right) m_{e} c^{2}
$$

We can project the expression of Eq.I.76 on the perpendicular and in the parallel axes

$$
\begin{aligned}
\mathrm{p}_{e, \perp} & =e A \\
\frac{\partial}{\partial t} \mathrm{p}_{e, x} & =\frac{\partial}{\partial x}(e \Phi)+\mathrm{F}
\end{aligned}
$$

Using Eq.I.74, the expression of the relativistic ponderomotive force can be found deriving Eq.I.77 expression

$$
\begin{aligned}
\mathbf{F}_{N A-r-\text { Pond }} & =-\frac{\partial}{\partial x}\left(\sqrt{m_{e}^{2} c^{4}+\mathbf{p}_{e}^{2} c^{2}}-m_{e} c^{2}\right) \\
& =\left[-\mathrm{v}_{e, x} \frac{\partial}{\partial x} \mathrm{p}_{e, x}-\frac{1}{2 \gamma_{e} m_{e}} \frac{\partial}{\partial x}\left(\mathrm{p}_{e, \perp}\right)^{2}\right] \mathbf{e}_{x}
\end{aligned}
$$

According to Eq.I.78 and knowing that we are using a linearly polarized laser propagating in the $\mathrm{x}$ direction with a laser vector potential $\mathbf{A}=A_{0} \cos (\varphi)$, with $\varphi=\omega_{L} t-k x$ and using the trigonometrical relation $\cos (2 \varphi)=2 \cos ^{2}(\varphi)-1$, we can demonstrate that

$$
\mathbf{F}_{N A-r-P o n d}=\left[-\mathrm{v}_{e, x} \frac{\partial}{\partial x} \mathrm{p}_{e, x}-\frac{e^{2}}{4 \gamma_{e} m_{e}} \frac{\partial}{\partial x}\left\{A_{0}^{2}\left[1+\cos \left(2 \omega_{L} t\right)\right]\right\}\right] \mathbf{e}_{x}
$$

The relativistic ponderomotive force is given by averaging Eq.I.82 over a laser oscillation and written

$$
\mathbf{F}_{r-\text { Pond }}=\left\langle\mathbf{F}_{N A-r-\text { Pond }}\right\rangle=-\frac{e^{2}}{4 \gamma_{e} m_{e}} \frac{\partial}{\partial x} A_{0}^{2} \mathbf{e}_{x}
$$

According to [Quesnel and Mora, 1998] we can also write the averaged relativisitc ponderomotive force as

$$
\mathbf{F}_{r-\text { Pond }}=\left\langle\mathbf{F}_{N A-r-\text { Pond }}\right\rangle=-m_{e} c^{2} \nabla\left\langle\gamma_{e}\right\rangle
$$

In conclusion and according to [Gibbon, 2005], the relation between $\mathrm{p}_{\perp}$ and $\mathrm{p}_{x}$, respectively the perpendicular and parallel momentum is given by

$$
\mathrm{p}_{x}=\frac{\mathrm{p}_{\perp}^{2}}{2 m_{e} c}
$$

It is noteworthy to add that the final electron energy is given by

$$
\Delta U=\left(\gamma_{e}-1\right) m_{e} c^{2}
$$


Knowing that the laser energy is transferred to the electron via the multi-photon momentum transfer and that the parallel momentum is conserved implies

$$
\mathrm{p}_{x}=\frac{n \hbar k}{c}=\frac{\Delta U}{c}=\left(\gamma_{e}-1\right) m_{e} c
$$

The electron emission angle is then given by

$$
\tan \theta=\frac{p_{\perp}}{p_{x}}=\sqrt{\frac{2}{\gamma_{e}-1}}
$$

We can see from the last equation that the higher is $\gamma_{e}$ the less divergent is the emitted electron.

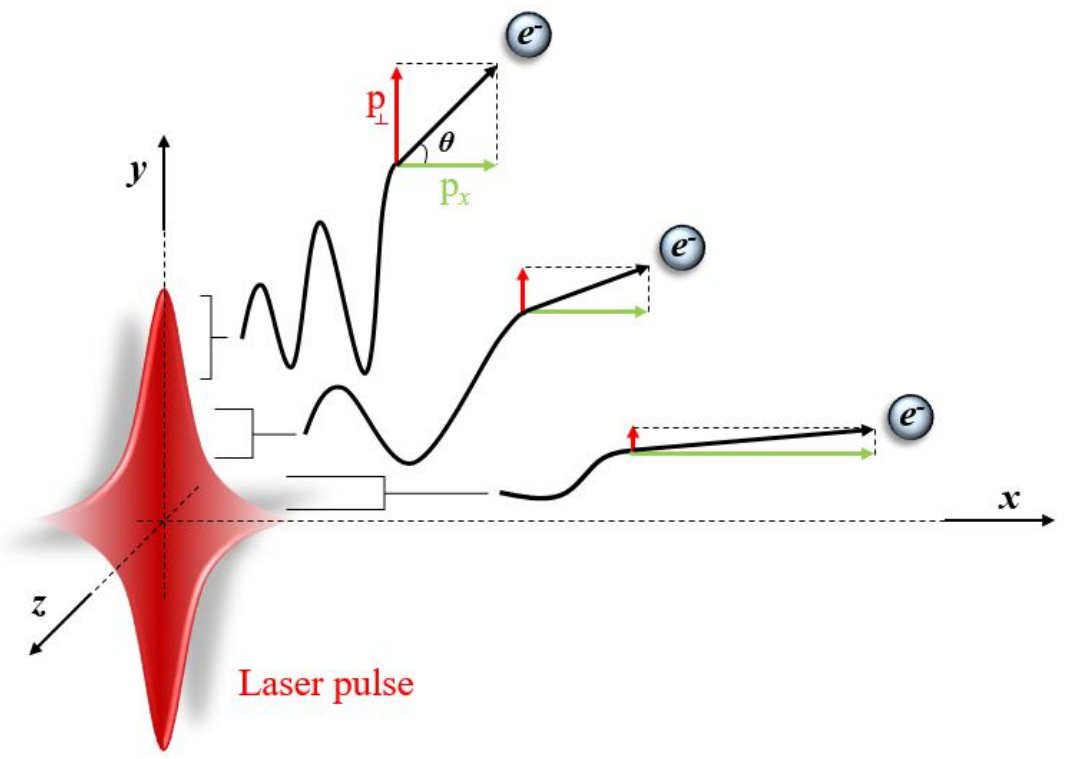

FIGURE I.15: Simplified scheme showing the relative ponderomotive effect over the electrons depending on the variation of the laser potential vector.

We can also note that the ponderomotive force is acting more strongly on electrons than on ions since it is inversely proportional to the mass of the particle. We can finally conclude that in the non-relativistic regime, the ponderomotive force is directed perpendicularly to the laser propagation while in the relativistic one, it is pushing more forward with the angle $\theta$ of Eq.I.88.

\section{I.2.6 Electron acceleration in the underdense plasma: Laser Wakefield Accelera- tion LWFA}

\section{I.2.6.a Electron trapping and acceleration in plasma waves}

As shown in the previous section, when a focused laser is propagating in a underdense plasma, electrons are separated from ions and then are kicked off from the laser propagation axis by the means of the ponderomotive force. This charge separation, produces restoring forces responsible for plasma waves generation. These plasma waves follow then the laser pulse during its propagation creating a wake in the back of the pulse called 'plasma wake' [Tajima and Dawson, 1979]. 
For an electromagnetic wave propagating in an under-dense plasma, phase and group velocities, $v_{p h}$ and $v_{g}$, can be calculated according to Eq.I.89 and Eq.I.90

$$
\begin{gathered}
v_{p h}=\frac{\omega_{L}}{k} \\
v_{g}=\frac{\mathrm{d} \omega_{L}}{\mathrm{~d} k}=\frac{c^{2}}{v_{p h}}=c \sqrt{1-\frac{\omega_{p e}^{2}}{\omega_{L}^{2}}}
\end{gathered}
$$

\section{Linear regime:}

In the linear regime where $a_{0} \ll 1$, in an initial uniform cold plasma, laser driven longitudinal plasma waves (by ponderomotive force) obey to the relations [Esarey et al., 2009, Krall et al., 1994]

$$
\begin{aligned}
& \left(\frac{\partial^{2}}{\partial t^{2}}+\omega_{p e}\right) \frac{\delta n_{e}}{n_{e, 0}}=c^{2} \nabla^{2} \frac{a^{2}}{2} \\
& \left(\frac{\partial^{2}}{\partial t^{2}}+\omega_{p e}\right) \Phi=\frac{\omega_{p e}^{2} a^{2}}{2}
\end{aligned}
$$

Where $n_{e, 0}$ is the background electron density and $\delta n_{e} / n_{e, 0}=\left(n_{e}-n_{e, 0}\right) / n_{e, 0}$ is the normalized density perturbation associated with the electrostatic wake $\Phi$ in the limit $a_{0}^{2} \ll 1$. The left-hand part of Eq.I.91 represents the density oscillation term of the plasma waves, while the right hand part corresponds to the wave driver i.e. the ponderomotive force in this case. The solutions for the density perturbation and the electric field of the wake are

$$
\begin{aligned}
& \frac{n_{e}}{n_{e, 0}}=\frac{c^{2}}{\omega_{p e}} \int_{0}^{t} d t^{\prime} \sin \left[\omega_{p e}\left(t-t^{\prime}\right)\right] \nabla^{2}\left(\frac{a\left(\mathbf{r}, t^{\prime}\right)^{2}}{2}\right) \\
& \frac{\mathbf{E}}{E_{0}}=-c \int_{0}^{t} d t^{\prime} \sin \left[\omega_{p e}\left(t-t^{\prime}\right)\right] \nabla\left(\frac{a\left(\mathbf{r}, t^{\prime}\right)^{2}}{2}\right)
\end{aligned}
$$

Where $E_{0}$ is the cold non-relativistic wave breaking field which is the peak field plasma waves can sustain in the linear regime.

$$
\begin{aligned}
& E_{0}=\frac{m_{e} c \omega_{p e}}{e} \\
& E_{0}[V / m]=96 \sqrt{n_{e}\left[\mathrm{~cm}^{-3}\right]}
\end{aligned}
$$

Thus, for typical densities of the order of $10^{18} \mathrm{~cm}-3$, the plasma can sustain fields of the order of $96 \mathrm{GV} / \mathrm{m}$.

In the linear regime $\left(a_{0} \ll 1\right)$, excited plasma waves have a perfect sinusoidal electric field shape following the laser pulse propagation. Thus, the density perturbations, caused by these plasma waves, have also a sinusoidal shape following the plasma waves electric field evolution presented in details in [Esarey et al., 2009, Lu et al., 2007].

The solutions of Eq.I.93 and Eq.I.94 show that the pulse length $c \tau_{L}$ of the driving laser (extent of the envelope $a^{2}$ ) should be in the order of the plasma wavelength $\lambda_{p}$ for optimum plasma wake 
generation efficiency given by

$$
c \tau_{L}=\frac{\sqrt{2 \ln (2)}}{\pi} \lambda_{p}
$$

This resonant condition has been achieved for LWFA experiments carried out in CLPU (see Experimental Section) where the typical densities in the order of $10^{18} \mathrm{~cm}^{-3}$ were used. Such densities correspond to plasma wavelengths of $\simeq 33 \mu \mathrm{m}$ (Eq.I.31), which means that laser pulses of the order of $40 \mathrm{fs}$ are needed (easily delivered by VEGA 2 laser system).

\section{Non linear regime:}

In the non linear regime $\left(a_{0} \gg 1\right)$, the laser field induces plasma waves of an electric field $E_{\mathrm{WB}}$ exceeding the value of $E_{0}$. According to [Esarey and Pilloff, 1995] $E_{\mathrm{WB}}$ is defined as the cold relativistic wave breaking field and is given by

$$
E_{\mathrm{WB}}=\sqrt{2}\left(\gamma_{p}-1\right)^{1 / 2} E_{0}
$$

Where $\gamma_{p}=\left(1-v_{\mathrm{p}}^{2} / c^{2}\right)^{-1 / 2}$ is the lorentz factor associated to the phase velocity of the plasma wave. We note that this one is approximately equal to the group velocity of the driver laser pulse in the 1D low intensity limit. In the non linear regime, the variation of density can no longer be described as perturbation, and relativistic effects must be taken into account. These non linear models are described in details in [Esarey et al., 2009, Sprangle et al., 1990]. It is true that analytical solutions in 1D approximation are not enough to explain all the physics of plasma waves excitation but are still of a significant interest to give an idea about what happens globally. In this regime, the electric field generated by the excited plasma waves is no longer sinusoidal and so the density perturbation becomes steeper. The non linear plasma period $\lambda_{p, n}$ becomes longer if compared to $\lambda_{p}$ and is expressed as

$$
\lambda_{p, n}=\lambda_{p} \times \begin{cases}1+3\left(E_{\max } / E_{0}\right)^{2} / 16, & E_{\max } \ll E_{0} \\ (2 / \pi)\left(E_{\max } / E_{0}+E_{0} / E_{\max }\right), & E_{\max } \gg E_{0}\end{cases}
$$

Where $E_{\max }$ is the peak electric field of the plasma waves. In this regime, the non linear aspect of the wake characteristics acquire, in addition to the laser intensity dependence, a transverse one. As a result of the non linear dependence of the plasma period on the wake amplitude, the wavefronts of the wake becomes curved, which makes the calculation analytically impossible, thus justifying the requirement of numerical calculation.

\section{Bubble regime:}

An extreme non linear case can take place when relativistically intense laser pulses are focused to spots of the order of the plasma wavelength. Via the ponderomotive force, the electrons are kicked away from the laser propagation axis in a specific regime called the "blow out", "cavitation" or "Bubble" regime (see Fig.I.16). The radially ejected electrons are attracted from the ions left behind thus creating a wake following the laser pulse. According to [Lu et al., 2007], the specific radius ("bubble" radius) of the wake must satisfy the following relation

$$
k_{p} R_{\text {bubble }}=k_{p} w_{0}=2 \sqrt{a_{0}}
$$


Where $w_{0}$ is the beam waist. The effective plasma wavelength in the bubble regime is then given by

$$
\lambda_{p, \text { bubble }}=2 R_{\text {bubble }}=\frac{2 \sqrt{a_{0}}}{\pi} \lambda_{p}
$$

This regime takes place for normalized laser potential $a_{0} \geq 4$, laser spots size $w_{0} \simeq R_{\text {bubble }}$, pulse

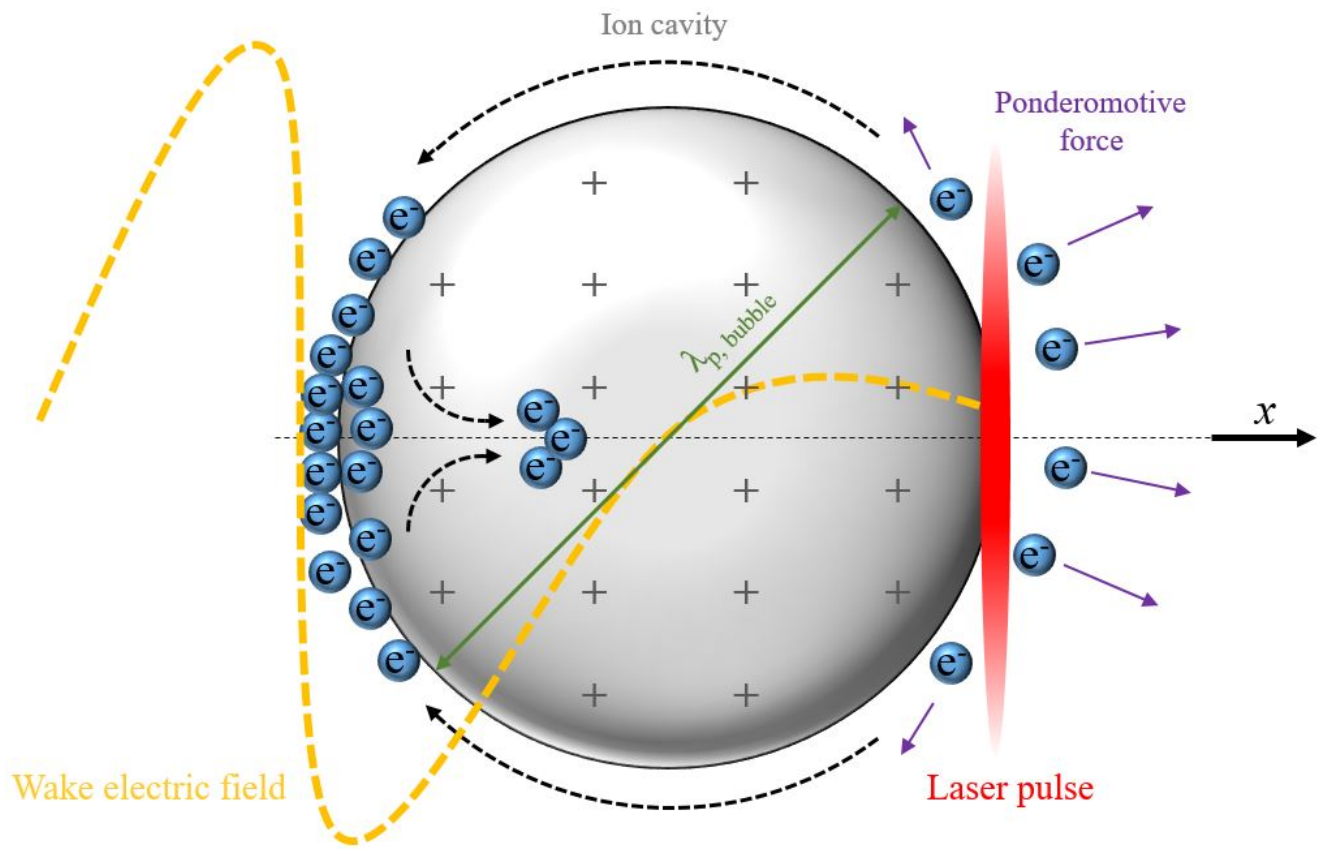

FIGURE I.16: Scheme for electron trapping and injection in the bubble regime.

durations $\tau_{L}<R_{\text {bubble }} / c=\left(\lambda_{p} \sqrt{a_{0}} / c\right) / \pi$ and corresponds to optimum wake generation. In this regime, the transverse focusing of electrons is maintained while the accelerating structure can propagate over many Rayleigh lengths.

In the left hand part of Fig.I.16, the electric filed of the plasma wake is increases linearly along the propagation axis $\mathrm{x}$ and goes to zero in the middle of the bubble. It is exactly in this half of the bubble where the electrons are accelerated. In the other side of the bubble, the inverse situation occurs and the electrons get decelerated due to the sign of the plasma wake. Furthermore, the plasma bubble also applies a transverse focusing force over the electrons obliging them to perform characteristic transverse movements known as "betatron" motion leading to a light emission in the x-ray range called "betatron" radiation (see more details about the betatron radiation further in this thesis).

\section{I.2.6.b Electron injection schemes in plasma wakes}

The huge electric fields generated in these ion cavities (bubble or ordinary plasma wake) open the possibility to accelerate electrons up to relativistic energies if they can be injected correctly in the accelerating structure. This mechanism can take place either spontaneously or in a controlled way. There exist many injection techniques [Corde et al., 2013, Malka, 2012, Faure, 2016] from which we present the following examples. 
- Self-injection: self injection (see [Mangles et al., 2012]) may take place spontaneously and can occur during different stages of the acceleration process. The trapped electrons can find themselves in a region of the bubble where the accelerating field is strong and thus get accelerated. An example of self injection in the bubble regime can be seen in Fig.I.16. Shot-to-shot stability in terms of, electron energies, charge, energy spread, divergence... is much lower in this regime if compared with other controlled injection techniques.

- Density ramp injection: This technique [Geddes et al., 2008] consists of decreasing the plasma wavelength by increasing the plasma density $n_{e}$ (Eq.I.31). Once increased, the plasma frequency will increase too (according to I.90) and thus the laser group velocity will decrease and so will be the case for the plasma wake velocity $\left(\mathrm{v}_{\text {ph,wake }} \simeq c\left(1-\omega_{p}^{2} / 2 \omega_{L}^{2}\right)\right)$. This will decrease dramatically the threshold velocity needed for the electrons to be trapped in the wake. Density down ramp injection can be carried out by adjusting the density profile of the gas used (special nozzles can be manufactured and purchased for this purpose).

- Ionisation induced: According to [Martinez de la Ossa et al., 2015], this technique consists of adding a second element called dopant to the gas mixture which gets ionised only when the mean laser pulse reaches its maximum intensity. The dopant has a higher ionising potential compared to the principal element of the gas. The electrons are then injected locally in the high electric field accelerating region of the wake. Mixing Nitrogen with pure Helium is one of many ionisation induced schemes for LWFA experiments.

- Colliding laser pulses: Consists of colliding a pump beam, responsible for wake driving, with a another laser propagating in the opposite direction generating a beat wave whose ponderomotive force can preaccelerate and locally inject background electrons into the wakefield [Esarey et al., 1997]. Adjusting the position of the latter, allows a better control on the electrons injection position and thus match the requirements for a given application.

\section{I.2.6.c Acceleration limits}

Many factors can affect the process of electron acceleration during LWFA [Faure, 2016]. Electrons can for example be injected into the wake up to a limit at which the electrostatic repulsion start to be strong enough to modify the shape of the wake and thus, its accelerating properties. This effect is usually called "beam loading". The latter determines the limits of charge a wake can sustain.

Laser diffraction is another factor affecting electron acceleration. Laser self focusing and laser diffraction are always present during laser propagation. An example to overcome this effect consists of generating pre-formed plasma channels which are meant for plasma guiding (electric discharges in gas cells is an example of plasma guiding). The laser diffraction effect is less important if compared with effects such as laser depletion or electron dephasing.

Laser pump depletion takes place when all the laser energy is transferred to the wake and thus, the acceleration stops after a specific distance called "depletion length" or "erosion length" [Lu et al., 2007] and is given for the bubble regime by

$$
L_{\mathrm{etch}} \simeq \frac{\omega_{p}^{2}}{\omega_{L}^{2}} c \tau_{L}
$$


Dephasing is another acceleration limiting factor and happens when electrons are accelerated to relativistic velocities (in the accelerating half part of the wake, where $E_{x}<0$ ). These relativistic electrons can escape and outrun the accelerating region of the wake. The distance at which this takes place is called dephasing length [Corde et al., 2013]. This one is given according to [Lu et al., 2007] for the blowout regime

$$
L_{\mathrm{deph}} \simeq \frac{2}{3} \frac{\omega_{L}^{2}}{\omega_{p}^{2}} R_{\text {bubble }}
$$

The maximum energy gain $\Delta E$ in this case is given by

$$
\Delta E[\mathrm{GeV}] \simeq 1.7\left(\frac{P[\mathrm{TW}]}{100}\right)^{1 / 3}\left(\frac{10^{18}}{n_{e}\left[\mathrm{~cm}^{-3}\right]}\right)^{2 / 3}\left(\frac{0.8}{\lambda_{L}[\mu \mathrm{m}]}\right)^{4 / 3}
$$

Quantities such as depletion and dephasing lengths for different non linear and bubble regimes are discussed in detail in [Esarey et al., 2009, Shadwick et al., 2009, Lu et al., 2007].

\section{I.2.7 Electron acceleration in the over dense plasma}

\section{I.2.7.a Target Normal Sheath Acceleration (TNSA) principle}

As seen in the previous section, laser light cannot propagate in overcritical density media. In these conditions, the laser energy is partiallly transmitted to the electrons prensent in the preformed plasma at the front surface of the target. These heated electrons transport the energy deeper in the target by colliding with ions which can be used for different purposes. Electron or Ion sources are very interesting for many applications such laser fusion, proton therapy, short xray sources and $\mathrm{x}$-ray imaging... TNSA is a very widely used technique for particle acceleration [Roth and Schollmeier, 2016] and is considered as one of the most effective ways to accelerate ions via ultra-short ultra-intense laser-thin foil interaction [Passoni, 2010, Passoni et al., 2013]. The standard scheme used for TNSA is shown in Fig.I.17.

An ulta-short and ultra-intense laser pulse is focused first into a micrometer scale size laser spot onto the target. The energy contained in the ASE of the laser pulse is generally enough to create a plasma on the front surface of the target. The main pulse arrives second and propagates inside the plasma up to the critical density level. It transmits a portion of its energy to the electrons of the media which will transport by themselves the energy deeper in the target [Sokollik, 2011]. For thin targets (few microns), the accelerated electrons can acquire enough energy to escape the rear side of the target and continue the propagation in vacuum. Ejected from the rear side, the gradient of electric field (positive charges left by the missing electrons) is enough to accelerate the ions (coming from impurities [Maksimchuk et al., 2000]) localised at backside of the target. Thus, both electrons and ions are accelerated [Clark et al., 2000]. It is noteworthy to add that electrons whose energy is not sufficient enough to leave the target are re-injected [Volpe et al., 2013] in the target, recirculate and can emit x-rays in the soft range (a few keV). 


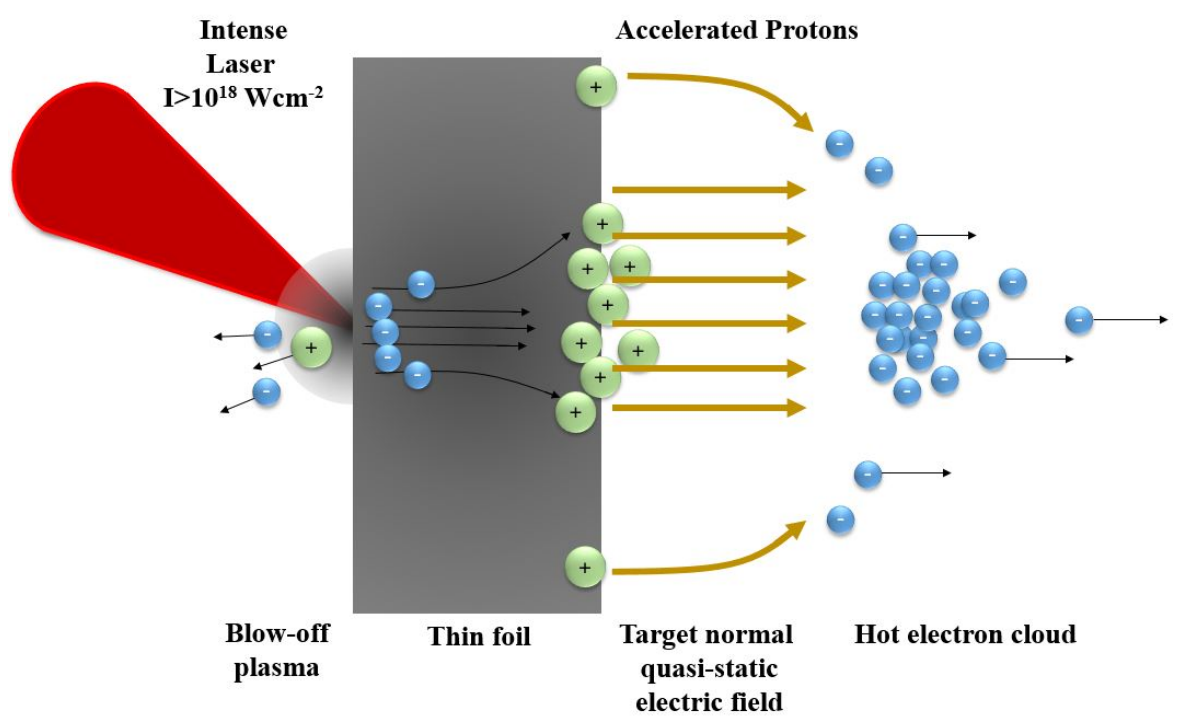

FIGURE I.17: Scheme inspired from [Schwoerer et al., 2006] for proton and hot electron acceleration via TNSA mechanism.

\section{I.2.7.b Introduction to fast electrons}

We have qualitatively described the ability of the ponderomotive force to generate high energy electrons beams. One way to measure laser absorption is basically to quantify the amount of energy transfered to these electrons. However, measuring the energy distribution of an electron beam is not a straight forward mission. Usually, for a direct measurement (destructive one), an electron spectrometer is needed. This diagnostic basically consists of applying a strong field (which can be electrical or magnetic), in the exact path of the accelerated electrons, what leads to a deviation in their trajectories. These deviations are proportional to the incident electron energy, thus, a full reconstruction of the electron beam energy distribution can be then achieved. In the Laser-solid interaction, it is important to know that these measurements are not fully correct since only the high energy electron beam population (> hundreds of $\mathrm{keV}$ ) is able to escape the target. These relativistic electrons are usually called "fast electrons" or "high temperature electrons". The low energy (or low temperature) electrons are mainly re-injected (trapped) into the target because of the presence a strong electric field generated at the rear side of target where there is a lack of electron density. This effect is known as electron refluxing or re-circulation as described in detail in [Volpe et al., 2013, Sentoku et al., 2003, Neumayer et al., 2010]. On the other hand, it exists another indirect method to carry out measurements for electron distribution characterisation. The latter consists of characterising the x-ray emission (Bremsstrahlung and/or $\mathrm{K} \alpha$ ) generated by the fast electron transport in matter. The generated electron energy spectra is usually discribed by a Maxwell-Juttner distribution function $f(E)$ such:

$$
f(E) \propto \beta \gamma^{2} \exp \left(-\frac{E}{T_{\mathrm{h}}}\right)
$$

Where $E$ is the hot electron energy with $E=(\gamma-1) m_{e} c^{2}, \beta$ is the fast electron speed $\beta=v / c$ and $T_{\mathrm{h}}$ is the hot electron temperature. A two temperature behaviour model is commonly used for simulations to express the energy distribution function $f_{\text {total }}(E)$ of the electrons 
[Sawada et al., 2014] and is written:

$$
f_{\text {total }}(E) \sim(1-R) \times f_{\text {hot- }}\left(E, T_{\text {hot- }}\right)+R \times f_{\text {hot }}\left(E, T_{\text {hot }}\right)
$$

Where $R$ is a scale factor the hot energy component and

$$
f(E, T) \sim \frac{1}{k_{B} T} \times \exp \left(-\frac{E}{k_{B} T}\right)
$$

Here $k_{B}$ is the Bolzmann constant, $E$ is the energy of the electrons and $T$ is the electron temperature. The two values $T_{\text {hot- }}$ and $T_{\text {hot }}$ correspond to the low and high energy components of the hot electron beam distribution. Many studies have been carried out to scale the hot electron temperature with the laser intensity $I$ or with the parameter $I \lambda^{2}$. The team of [Beg et al., 1997, Haines et al., 2009] have proposed an empirical law valid for intensities $I \leq 10^{19} \mathrm{Wcm}^{-2}$ known as Beg's law and is given:

$$
T_{\text {hot }}^{\text {Beg }}[\mathrm{keV}]=215\left(I_{18} \lambda_{L(\mu \mathrm{m})}^{2}\right)^{1 / 3}
$$

On the other side, for higher intensities, such as $10^{18} \leq I \leq 10^{21}$, Wilks team has proposed a scaling law [Wilks et al., 1992, Gibbon, 2005] written:

$$
T_{\text {hot }}^{\text {Wilks }}[\mathrm{keV}]=511\left(\sqrt{1+0.73 I_{18} \lambda_{L(\mu \mathrm{m})}^{2}}-1\right)
$$

Fig.I.18 is showing graphically a comparison between Beg and Wilks high temperature electron scaling laws. 


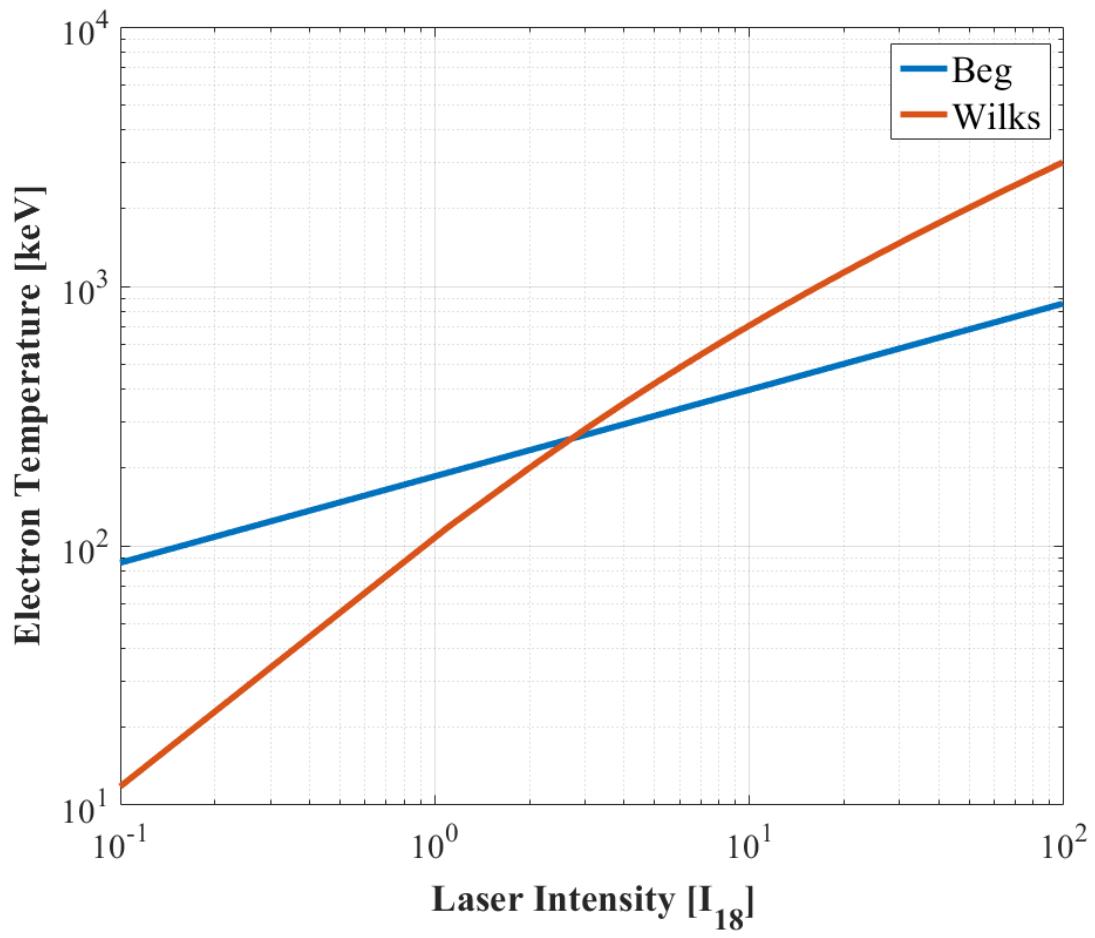

FIGURE I.18: Comparison between both scaling laws for hot electron temperature. 


\section{Chapter II}

\section{Laser driven X-ray sources}

\section{Introduction}

$\mathrm{X}$-rays define the highly energetic electromagnetic radiation, in the range of few hundreds of eV up to $100 \mathrm{keV}$, and are used since the $19^{\text {th }}$ century [Spiegel, 1995] up to nowadays in practically all domains (biology, medicine, physics, chemistry...) mainly for imaging and probing. The oldest, simplest and controllable x-ray source is the x-ray tube, and it was discovered at the beginning of 20th century. These ones are known for their beam reproducibility, flexibility for experimental layouts and small cost but can only deliver a low x-ray flux (see comparison in Fig.II.1). In the early 70's, synchrotron facilities have appeared. The latter have proven to be very reliable for generating controlled x-ray beams. Such facilities deliver x-ray pulses of high brilliance (a quantity defined as the number of photons emitted per solid angle per unit of time per $0.1 \%$ bandwidth) orders of magnitude higher than the one obtained with $\mathrm{x}$-ray tubes. New generation of XFEL (4th X-ray Free Electron Lasers) are even more powerful and deliver fully coherent [Geloni et al., 2010] x-ray beams (temporally and spatially coherent) with peak brilliance higher than anything ever achieved (see Fig.II.1). With these beam characteristics, many applications (x-ray diffraction based, nano-scale imaging, etc...) can be carried out in such facilities. However, both the cost (hundred of million Euros) and the size (a few $\mathrm{km}$ ) of such installations are considerable. The fast development of laser technology, together with the development of high precision targetry systems, have opened the possibility to have other alternative x-ray sources, based on the interaction of focused lasers (multi TW class) with matter. This chapter (and thesis) will be mainly dedicated to laser-driven $\mathrm{x}$-ray sources such as $\mathrm{K} \alpha$, betatron and Bremsstrahlung radiation. Examples of generation and properties of laser driven $\mathrm{x}$-ray radiation are discussed. A section about the basics of $\mathrm{x}$-ray matter interaction theory is also introduced in this chapter for understanding the fundamentals of x-ray optics.

\section{Overview: temporal and spatial coherence}

The best way to charachterise x-ray sources is based on their spatial and temporal coherence properties [Saleh and Teich, 2007]. We can define coherence as the measure of correlation between the phases measured in different points on a wave. We refer to the spatial or temporal coherence by considering the correlation measurement of phase in the transverse or in the longitudinal directions respectively. Fig.II. 2 shows the different possible cases of coherence a radiation can have. In other words, temporal coherence allows the measurement of source monochromatic- 


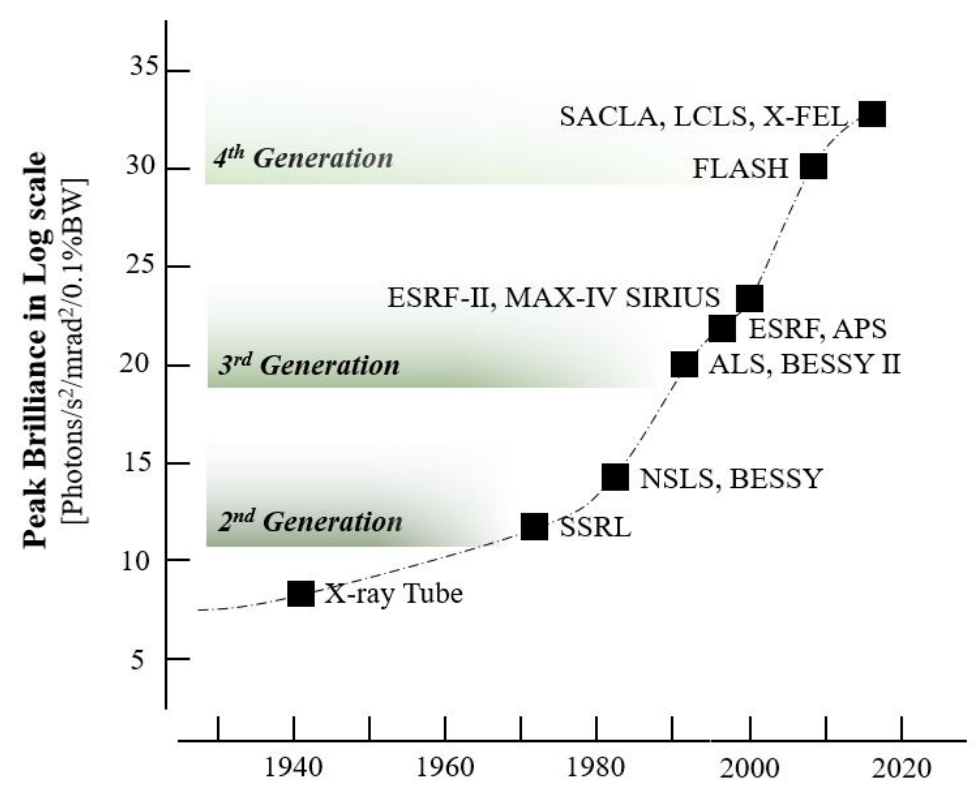

FIGURE II.1: Brilliance map as a function of years for different $\mathrm{x}$-ray sources. Inspired form [Eberhardt, 2015].

\section{Wavefronts}

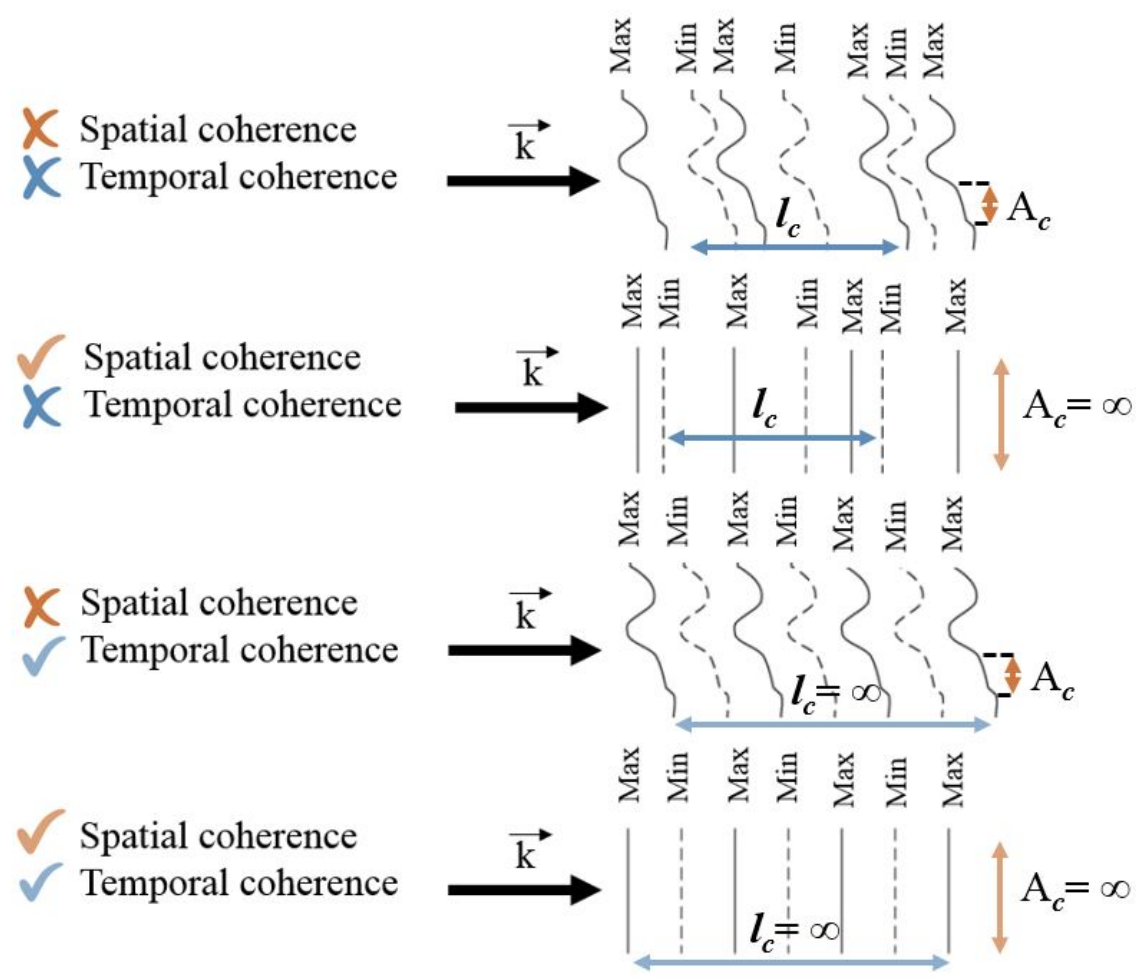

FIGURE II.2: Different cases for radiation coherence. Examples of coherence lengths $l_{c}$ and coherence areas $A_{c}$ (defined later) are also shown. 
ity. Let us define a function $G(\tau)$ as the convolution of a signal $U(t)$ with a delayed copy of itself $U(t+\tau)$ as a function of delay $\tau$. In practice, this operation is nothing but the autocorrelation carried out daily in laser laboratories to know the duration of isolated laser pulses. $G(\tau)$ is then written

$$
G(\tau)=\int_{-\infty}^{+\infty} U^{*}(t) U(t+\tau) d t=\int_{-\infty}^{+\infty} U(\tau) U^{*}(t-\tau) d t
$$

According to [Saleh and Teich, 2007], we can define the coherence time $\tau_{c}$ and coherence length $l_{c}$ as

$$
\begin{gathered}
\tau_{c}=\int_{-\infty}^{+\infty}|g(\tau)|^{2} d \tau \\
l_{c}=c \tau_{c}
\end{gathered}
$$

Where $g(\tau)$ is the degree of coherence given by

$$
g(\tau)=\frac{G(\tau)}{G(0)} \quad 0 \leq|g(\tau)| \leq 1
$$

On the other hand, spatial coherence is defined as a measurement of correlation between the phase of two points in the transverse dimension for a given wave. The latter is said to be perfectly spatially coherent if it has the same value of amplitude over an infinite length. In other words, spatial coherence is giving information about uniformity of the wavefront phase. We define $A_{c}$, area over which exists a significant degree of coherence. According to Van Cittert-Zernike theorem [Thompson et al., 2017], the spatial coherence area is given by

$$
A_{c}=\frac{D^{2} \lambda^{2}}{\pi d^{2}}
$$

Here $d$ is the diameter of the light source and $D$ is the distance away. $A_{c}$ gets larger as far as we get away from the source. Also, wavefronts gets smoother as they propagate away from the source. The Young's double slit interferometer is a useful tool to demonstrate and measure the spatial coherence. This property is fundamental for $\mathrm{x}$-ray phase contrast imaging experiments. More details are found further in this thesis (see Sec.IV.4.1).

The different $\mathrm{x}$-ray sources presented in this thesis will be then cited according to their degree of coherence. On the one side, both K-shell emission and bremsstrahlung will be presented as fully incoherent radiation. In the other side, betatron emission, generated via LWFA will be considered as partially (spatially) coherent. 


\section{II.1 Types of X-ray sources}

\section{II.1.1 K $\alpha$ emission}

\section{II.1.1.a Generation}

Accelerated, fast electrons interact with the atoms of the media in which they propagate. During this process, fast electrons transfer/deposit their energy via collisions to the surrounding atoms. An incident electron with enough energy, $E$, can excite a bound electron from the ground state $E_{i}$ to an excited one $E_{j}$ if $E>\left|E_{j}-E_{i}\right|$. If it is desirable to discriminate fast electrons from the thermal ones, higher possible atomic transitions should be considered. The highest energy transition corresponds to the ionisation of the electron of the most inner shell of the atom, "K", characterized by the quantum number $n=1$ of the orbital $1 s$. The ionisation can occur only if the energy of the incident electron is higher than the binding energy of the K-shell, $I_{K}$. According to [Casnati et al., 1982], for an atomic number $Z<92, I_{K}$ is given approximately by

$$
\frac{I_{K}}{E_{R y}}=0.424 Z^{2.1822}
$$

where $E_{R y}$ is the Rydberg energy $\left(E_{R y}=m_{e} e^{4} / 8 \varepsilon_{0}^{2} h^{2} \sim 13.60593 \mathrm{eV}\right)$. During a collision event electrons of sufficient energy can eject bound ones situated in the inner shell of atoms. The ejected electron leaves an empty gap, so, the ionised atom decays to more stable states by filling this gap with outer shell electrons (ones from $L$ and $M$ shells). This leads to an Auger effect, where the transition energy is transferred to an other atomic electron (Auger electron), or to an $\mathrm{x}$-ray photon with the specific transition energy. This $\mathrm{x}$-ray fluorescence process is called $\mathrm{K} \alpha$ emission if the transition is from $2 p$ to $1 s$ (from L-shell to K-shell). Fig.II.3 is showing the entire process for $\mathrm{K} \alpha$ emission.
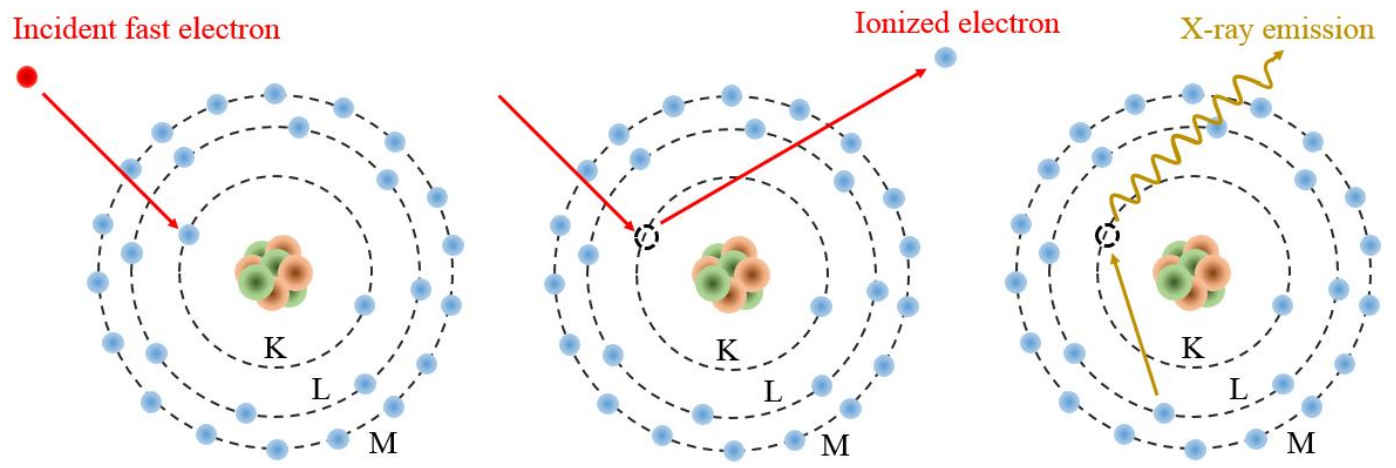

FIGURE II.3: Scheme for K-shell emission process.

$\mathrm{K} \alpha_{1}$ and $\mathrm{K} \alpha_{2}$ constitute the two possible transitions for the $\mathrm{K} \alpha$ fluorescence emission. $\mathrm{K} \alpha_{1}$ refers to the transition $2 p_{1 / 2} \rightarrow 1 s$ meanwhile $\mathrm{K} \alpha_{2}$ corresponds to the transition $2 p_{3 / 2} \rightarrow 1 s$. These two transitions occur following the quantum selection rules. For a given transition between two energy levels, the conditions to satisfy are $\Delta l= \pm 1$ and $\Delta j= \pm 1 ; 0$, with $l$, and $j$ are respectively the orbital and the total angular quantum number. 
Less probable than $\mathrm{K} \alpha, \mathrm{K} \beta$ emission is another possible transition to the $\mathrm{K}$-shell level. $\mathrm{K} \beta$ emission corresponds to the transition (M-shell to K-shell) $3 p \rightarrow 1 s$. We note that $\mathrm{K} \beta$ emission probability decreases relatively to the $\mathrm{K} \alpha$ one, when a sample is heated (due to successive ionisations). Fig.II.4 is presenting the possible K-shell transitions for a copper atom.

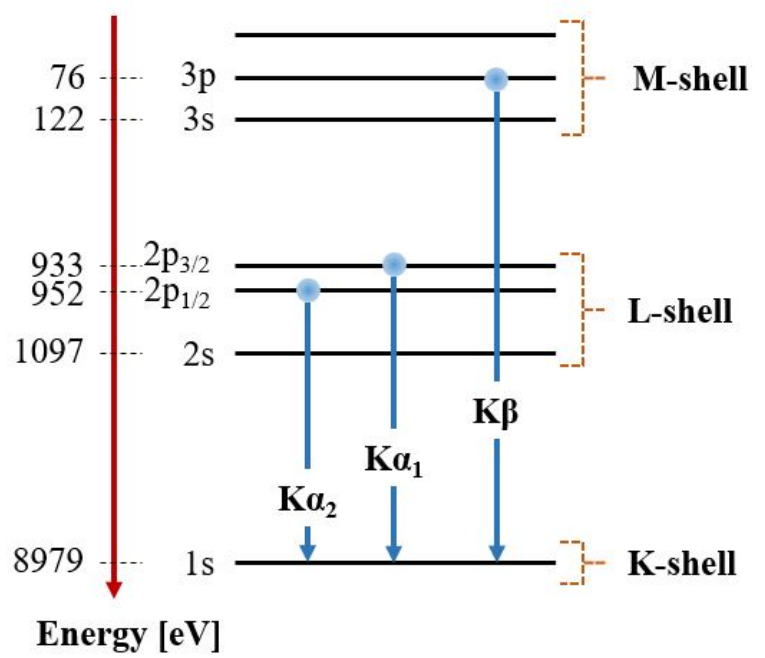

FIGURE II.4: K-shell emission transitions for a cooper atom.

The energy of the K $\alpha$ transition can be estimated using the empirical Moseley's [Moseley, 1913] for atoms of atomic number $3<Z<60$. This model does not separate $\mathrm{K} \alpha_{1}$ emission from $\mathrm{K} \alpha_{2}$ one and is given by

$$
E_{K \alpha}=10.206(Z-1)^{2}
$$

Fig.II.5 shows the evolution of both energies of binding and $\mathrm{K} \alpha$ emission in function of the atomic number $Z$ for atoms of $3<Z<60$. According to [Moseley, 1913], the binding energy function has a maximum error of $4 \%$, while the energy function for $\mathrm{K} \alpha$ emission has a maximum error of $5 \%$.

\section{II.1.1.b $\quad \mathrm{K} \alpha$ emission cross section}

Writing $\sigma_{\text {ion }}$ as the K-shell ionization cross section and $f_{K}$, as the fluorescence yield, the K-shell emission cross section $\sigma_{K}$ is given by [Casnati et al., 1982] as

$$
\sigma_{K}=\sigma_{i o n} f_{K}
$$

[x-ray data boklet, ] and [Krause, 1979] define $f_{K}$ as the probability of filling a core hole in the $\mathrm{K}$-shell by a radiative process (in competition with non radiative processes, Auger electron). We note that emission of an Auger electron is the only non radiative process competing with the fluorescence $\mathrm{K}$. Many expressions of probability of deexcitation by $\mathrm{K} \alpha$ emission, $f_{K}$, as a function of the atomic number $Z$ (for $6 \leq Z \leq 99$ ) have been estimated by fitting experimental results in a considerable number of publications as summarized in [Kahoul et al., 2011]. One of 


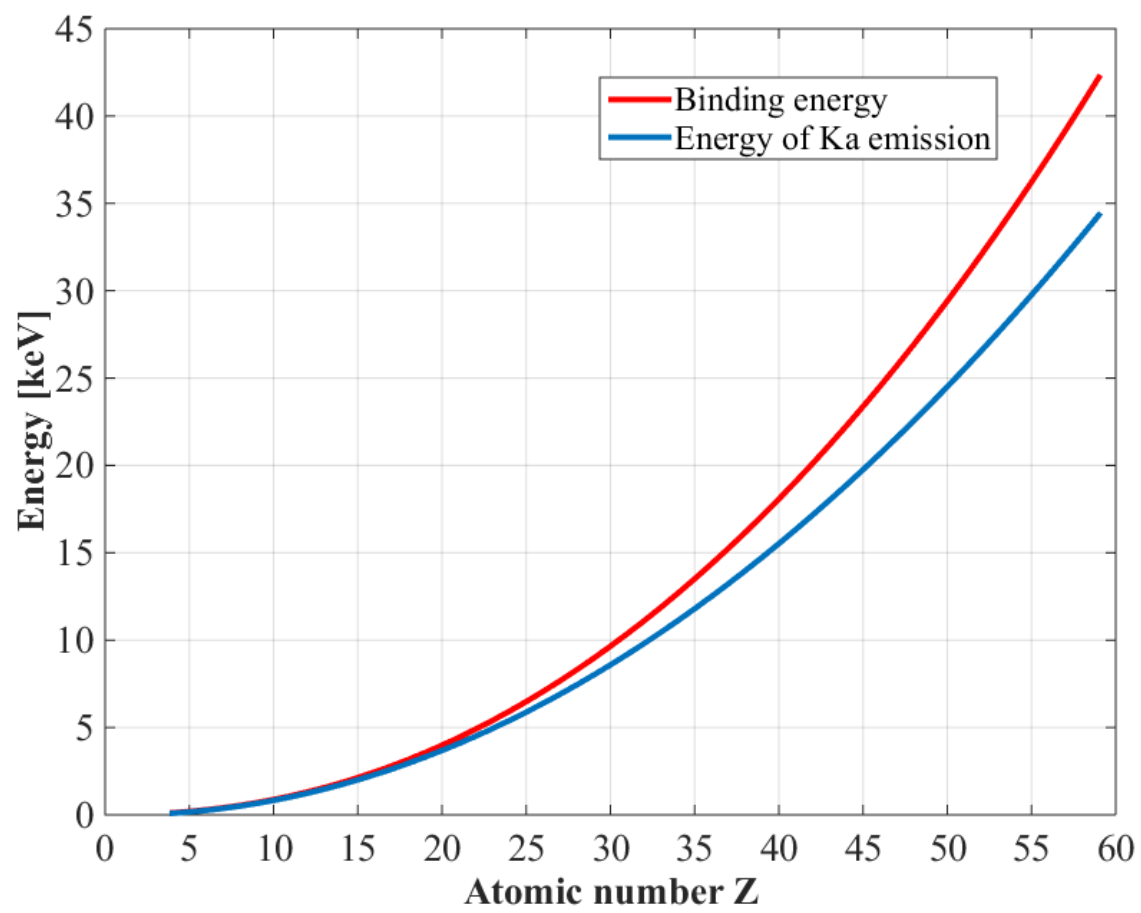

FIGURE II.5: Binding energy $I_{K}$ and $K \alpha$ emission energy $E_{K \alpha}$ in function of the atomic number for $3<\mathrm{Z}<60$.

the best semi empirical formulas fitting the experimental data for $f_{K}(Z)$ is given by

$$
f_{K}(Z)=\frac{\left[\sum_{i=0}^{3} a_{i} Z^{i}\right]^{4}}{1+\left[\sum_{i=0}^{3} a_{i} Z^{i}\right]^{4}}
$$

where

$$
\begin{aligned}
& a_{0}=0.024 \pm 0.074 \\
& a_{1}=0.030 \pm 0.005 \\
& a_{2}=(1.081 \pm 1.136) 10^{-4} \\
& a_{3}=(-4.633 \pm 1.281) 10^{-6}
\end{aligned}
$$

The fluorescence yield function $f_{K}(Z)$ for elements of atomic number $Z, 5 \leq \mathrm{Z} \leq 99$, is plotted in Fig.II.6.

It is worth to note that the Auger process is dominant for low $Z$ elements and decreases strongly if compared to the $\mathrm{K} \alpha$ emission process for high $Z$ elements. 


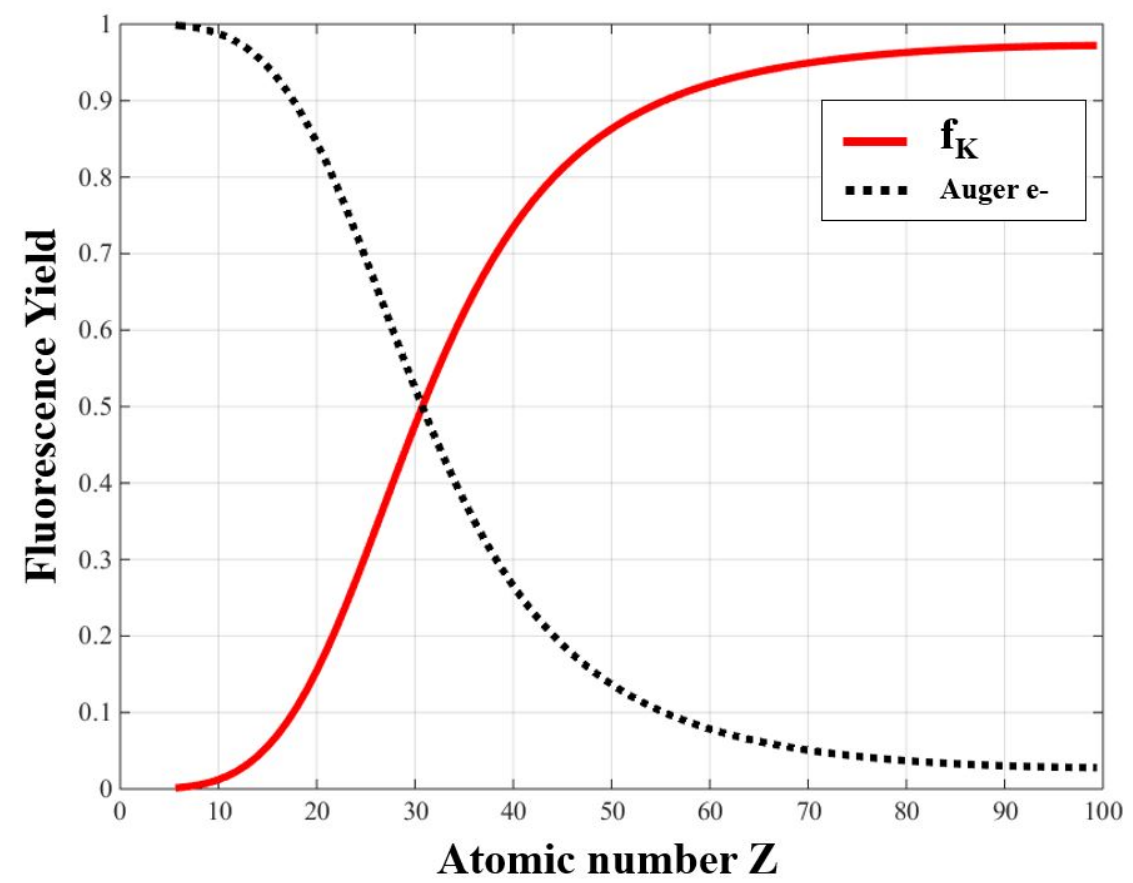

FIGURE II.6: Fluorescence yield of the K-shell $f_{K}$ for $5 \leq \mathrm{Z} \leq 99$.

According to the empirical model of Hombourger described in [Hombourger, 1998], the ionisation cross section $\sigma_{i o n}$ is written as

$$
\sigma_{\text {ion }}=n_{K} \pi a_{B}^{2} G_{r}\left(\frac{E_{R y}}{I_{K}}\right)^{C_{U}} D_{U}
$$

where $n_{K}$ is the number of electrons of the K-shell (basically 2), $a_{B}$ is the first Bohr radius $\left(a_{B}=\varepsilon_{0} h^{2} / \pi m_{e} e^{2}\right), G_{r}$ is the relativistic factor of Grysinski (taking into account the relativistic effect in the incident electron motion) and is written as

$$
G_{r}=\left(\frac{1+2 J}{U+2 J}\right)\left(\frac{U+J}{1+J}\right)^{2}\left(\frac{(1+U)(U+2 J)(1+J)^{2}}{J^{2}(1+2 J)+U(U+2)(1+J)^{2}}\right)^{3 / 2}
$$

where $J=\left(m c^{2} / I_{K}\right)$ and $U=E / I_{K}$. Here $E$ is the energy of the incident electron. $D_{U}$ and $C_{U}$ are two dimensionless factors depending on $U$ where

$$
\begin{gathered}
D_{U}=\left(3.125-\frac{4.172}{U}+\frac{1.877}{U^{2}}\right) \frac{\ln (U)}{U} \\
C_{U}=2.0305-\frac{0.316}{U}+\frac{0.1545}{U^{2}}
\end{gathered}
$$

Examples of calculated ionisation cross section $\sigma_{i o n}$ as a function of incident x-ray photon energies, for atoms of different $Z$ are shown in Fig.II.7.

According to Fig.II.7, we see how $\sigma_{i o n}$ is evolving as a function of the atomic number Z. We can also note from Fig.II.7, how $\sigma_{i o n}$ increases just after the K-shell binding energy (so called 

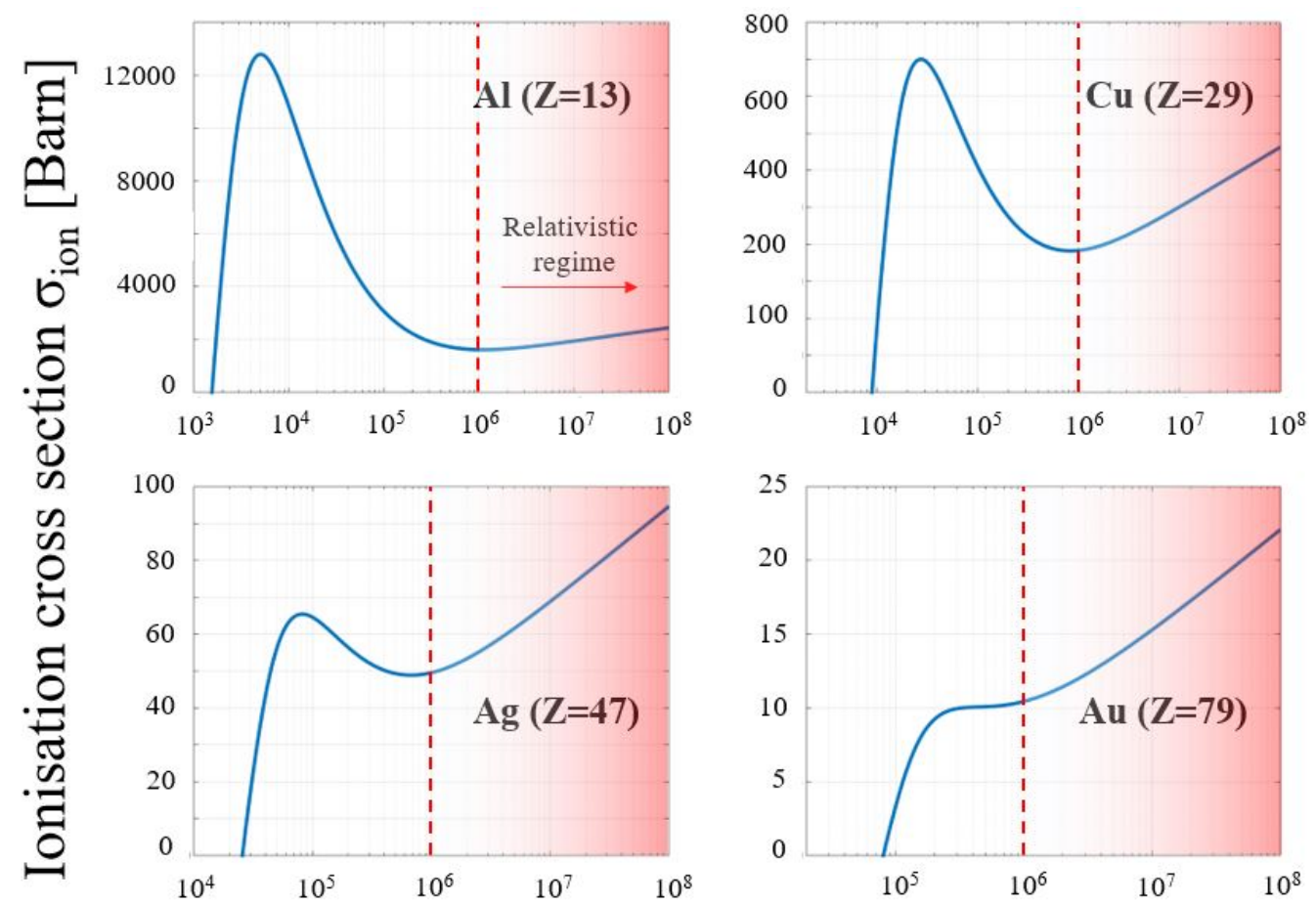

\section{Incident electron energy in $[\mathrm{eV}]$}

FIGURE II.7: Calculated ionization cross section $\sigma_{i o n}$ of the K-shell by electron impact for aluminium, cooper, silver and gold as a function of electron energy.

K-edge) and decreases until a local minimum for materials of $Z \sim 60$. This behaviour disappears gradually for atoms of high $Z$ (case of gold seen in Fig.II.7 (bottom right)).

\section{II.1.1.c $K \alpha$ photon number}

Let's consider a laser-solid interaction for relativistic intensities where $\mathrm{I}>10^{18} \mathrm{~W} \cdot \mathrm{cm}^{-2}$. According to [Malka and Miquel, 1996], the high energy tail of the electron distribution is described by a Boltzmann distribution in the form

$$
f(E) \sim \exp \left(-\frac{E}{T_{h}}\right)
$$

where $T_{h}$ is the hot electron temperature. According to [Salzmann et al., 2002], the total number of $\mathrm{K} \alpha$ photons emitted in both, forward and backward directions (see Fig.II.8), is given by

$$
\begin{aligned}
N_{K \alpha}=N_{h} n_{A} f_{K} \frac{\Omega_{D}}{4 \pi} \frac{\lambda_{\mathrm{mfp}}}{T_{h}} \int_{0}^{\infty} d E \sigma_{K}(E) \int_{0}^{d} d x & f\left[E_{0}(E, x)\right] \\
& \times \begin{cases}\exp \left(-\frac{x}{\lambda_{\mathrm{mfp}}}\right) & \text { backward direction } \\
\exp \left(-\frac{d-x}{\lambda_{\mathrm{mfp}}}\right) & \text { forward direction }\end{cases}
\end{aligned}
$$




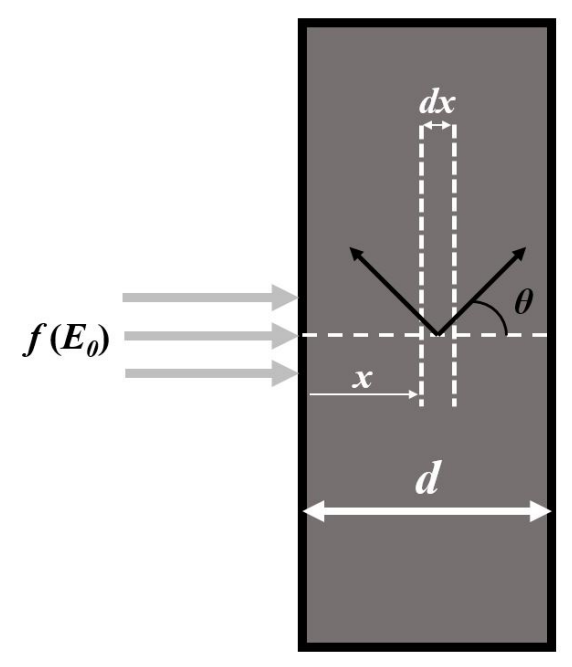

FIGURE II.8: 1D Scheme for $\mathrm{K} \alpha$ generated via the interaction with an electron population of initial energy $E_{0}$ in a thin target. Figure inspired from

[Salzmann et al., 2002].

Where $N_{h}$ is the number of hot electron passing through the target, $n_{A}$ is the atomic number density, $f_{K}$ and $\sigma_{K}$ are the $K \alpha$ fluorescence yield and the ionisation cross section defined in the previous section. $\Omega_{D}$ represents the solid angle over which the $\mathrm{K} \alpha$ photons are emitted. The argument of $f, E_{0}(E, x)$ refers to the electrons of energy $E$ and situated at a depth $x$ with an initial energy $E_{0} . \lambda_{\mathrm{mfp}}$ is an angle dependent photon mean free path and its expression is given by

$$
\lambda_{\mathrm{mfp}}=\frac{|\cos (\theta)|}{n_{A} \sigma_{p h}\left(h v_{K}\right)}
$$

where $\sigma_{p h}\left(h v_{K}\right)$ is the photo absorption cross section for a $\mathrm{K} \alpha$ photon.

\section{II.1.2 Bremsstrahlung emission}

Bremsstrahlung means "breaking radiation" or "decelerating radiation", it occurs when there is a collision between two charged particles of different species (see Fig.II.9). In this thesis, we consider that the Bremsstrahlung is mainly emitted by the coulomb interaction of the accelerated hot electron with the plasma background ions. Bremsstrahlung is a continuous broadband emission. It is generated along a cone angle centered on the electrons direction and highly dependent of the electrons incident energy by $\sim 1 / \gamma$ ( $\gamma$ is the Lorentz factor), i.e the lower the incoming electrons energy is, the wider the opening cone angle of the the Bremsstrahlung is and vice versa. Analysing the Bremsstrahlung emission is a very important tool for characterizing the energy distribution of hot electron propagation in targets.

According to [Jackson, 1962, Corde et al., 2013], one can get from the so called LineardWiechert electromagnetic field, the general expression giving the radiation emitted by an electron in the direction of observation $\vec{n}$, as a function of its position, velocity and acceleration along the 


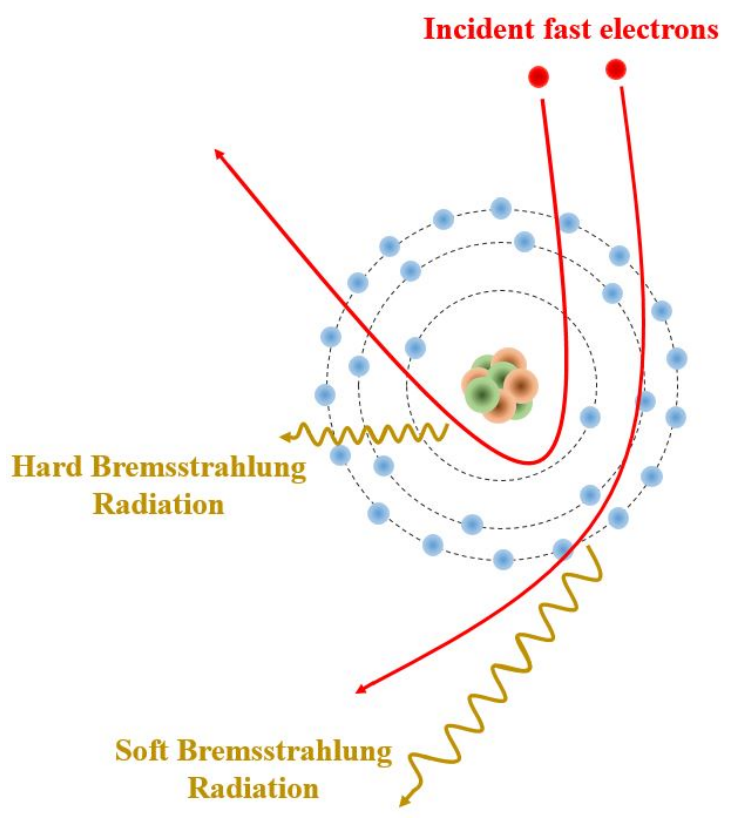

FIGURE II.9: Generation of hard and soft bremsstrahlung radiation.

trajectory

$$
\frac{d^{2} I}{d \omega d \Omega}=\frac{e^{2}}{16 \pi^{3} \varepsilon_{0} c} \times\left|\int_{-\infty}^{+\infty} e^{i \omega[t-\vec{n} \cdot \vec{r}(t) / c]} \frac{\vec{n} \times[(\vec{n}-\vec{\beta}) \times \dot{\vec{\beta}}]}{(1-\overrightarrow{\beta . \vec{n}})^{2}} d t\right|^{2}
$$

Eq.II.17 represents the energy radiated within a spectral range $d \omega$ centered on the frequency $\omega$, all this in the solid angle $d \Omega$ centered on the unit vector in the the observation direction $\vec{n}$. Here $\vec{r}$ is the position of the electron at time $t, \vec{\beta}$ is the electron velocity normalized to the speed of light, and $\dot{\vec{\beta}}=d \beta / d t$ is the electron acceleration divided by $c$. Eq.II.17 is valid for an observer far from the electron so that $\vec{n}$ is constant along the trajectory. Looking at Eq.II.17, a few comments can be made:

- We can see that if the acceleration is equal to zero $(\dot{\vec{\beta}}=0)$, the right side of Eq.II.17 is equal to zero. This confirms the emission of electromagnetic radiation from accelerated charged particles.

- The term $(1-\overrightarrow{\beta . \vec{n}})^{-2}$ shows that the radiated energy is maximum when the conditions $\vec{\beta} . \vec{n} \rightarrow 1$ (meaning that $\vec{\beta}$ and $\vec{n}$ are parallel) and $\beta \simeq 1$ are fulfilled. Thus the more relativistic are the electrons the more the emitted radiation (along the velocity direction) is important.

- Concerning the term $(\vec{n}-\vec{\beta}) \times \dot{\vec{\beta}}$ and according to [Corde et al., 2013], it is more efficient to generate the radiation if the force applied to the particle is transverse rather than longitudinal. We can also see that the radiated energy evolves with $\dot{\vec{\beta}}^{2}$, thus, the higher is the acceleration, the higher is the energy radiated. 


\section{II.1.3 Betatron radiation}

\section{II.1.3.a Electron oscillations in the ion cavity}

In the previous chapter, we have shown that it is possible to accelerate electrons in underdense plasmas. During the acceleration process, the wake continues to apply a restoring transverse forces, driving the electrons to oscillate transversally (wiggling around the laser propagation axis) as seen in Fig.II.10. These electron oscillations are responsible for the generation of radiation in the x-ray range called "Betatron" radiation, characterised by a broadband emission spectra and small divergence (few mrad solid angle). Fig.II.10 shows the main scheme for electron acceleration in an ion cavity. The most important parameters related to the betatron generation are also highlighted in the same figure.

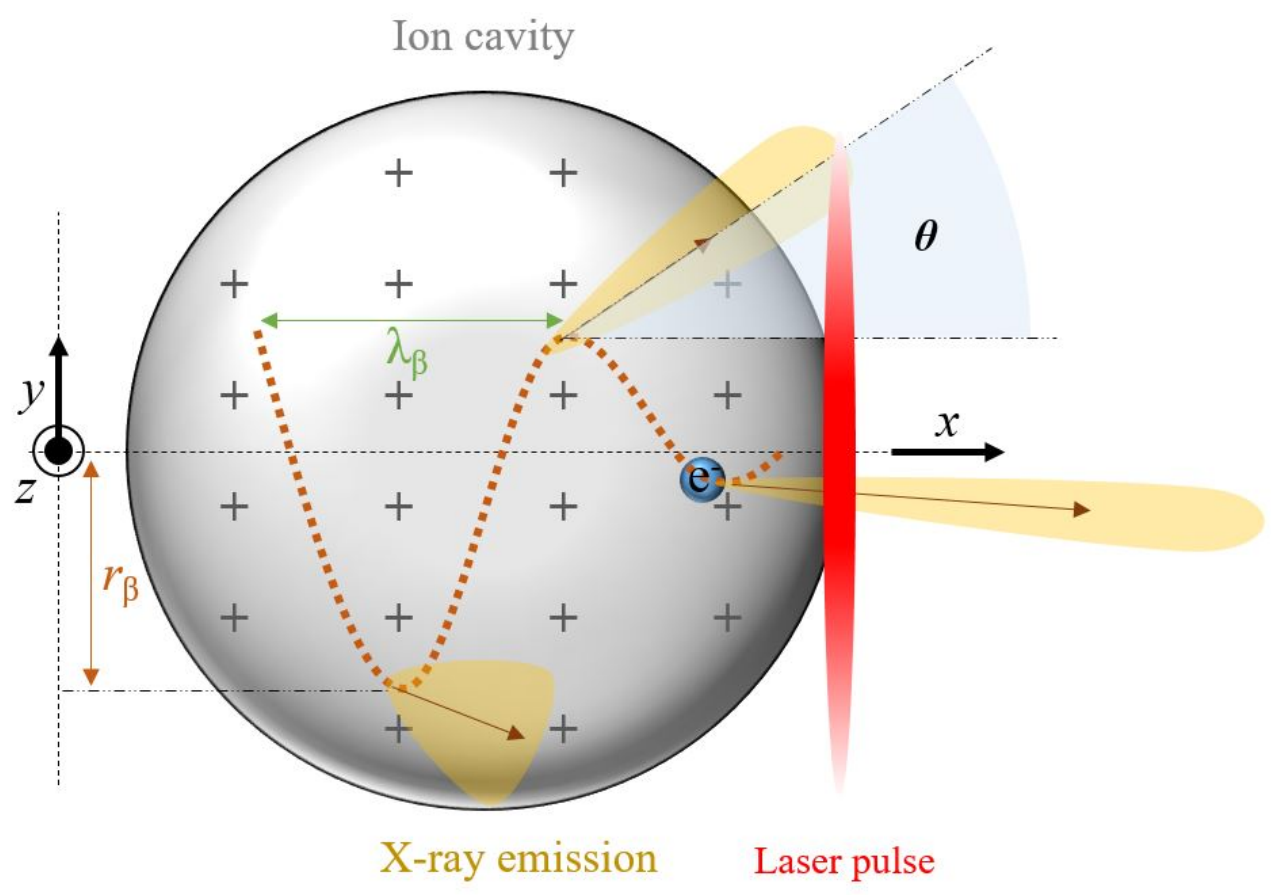

FIGURE II.10: Simple scheme for the generation mechanism of betatron radiation and its related parameters: $\lambda_{\beta}$ is the betatron wavelength, $r_{\beta}$ is the betatron amplitude and $\theta$ is the betarton divergence angle. Figure inspired from

[Corde et al., 2013].

The electrons inside the ion cavity (bubble) undergo two main forces. One is parallel to the laser propagation axis (the accelerating field of the bubble), and another one is radial (transverse). Let us consider only this latter and use Eq.I.28 to approximate the transverse electric field applied to the accelerated electron bunch $E_{r}$ as

$$
E_{r}=\frac{n_{e} e}{2 \varepsilon_{0}} r
$$

We know that in this case, the transverse momentum is very small compared to the longitudinal one $\left(\gamma \simeq \gamma_{x}>>\gamma_{r}\right)$, we can write the equation of movement, in the transverse coordinate, of an 
injected single electron inside an electric field $E_{r}$ (applied by the ion cavity) as

$$
\gamma m_{e} \frac{d^{2}}{d t^{2}} r=-e E_{r}
$$

We can rewrite Eq.II.19 in terms of plasma frequency $\omega_{p}$, getting

$$
\frac{d^{2} r}{d t^{2}}=-\frac{1}{2 \gamma} \frac{n_{e} e^{2}}{\varepsilon_{0} m_{e}} r=-\frac{1}{2 \gamma} \omega_{p}^{2} r=-\omega_{\beta}^{2} r
$$

Here we have introduced $\omega_{\beta}$, known as the betatron frequency

$$
\omega_{\beta}=\frac{\omega_{p}}{\sqrt{2 \gamma}}
$$

The betatron wavelength, $\lambda_{\beta}$, seen in Fig.II.10, is then equal to

$$
\lambda_{\beta}=\sqrt{2 \gamma} \lambda_{p}
$$

$\lambda_{\beta}$ is written in practical units as

$$
\lambda_{\beta}[\mu \mathrm{m}]=4.72 \times 10^{10} \sqrt{\frac{\gamma}{n_{e}\left[\mathrm{~cm}^{-3}\right]}}
$$

According to [Corde et al., 2013], and considering a propagation in the x-direction (same as shown in Fig.II.10) with electron oscillations in the perpendicular plane (y,z), the electron trajectories are sinusoidal and are given by

$$
\begin{aligned}
& y(t)=y_{\beta}(t) \cos \left(\int_{0}^{t} \omega_{\beta}\left(t^{\prime}\right) d t^{\prime}+\phi_{y}^{\prime}\right) \\
& z(t)=z_{\beta}(t) \cos \left(\int_{0}^{t} \omega_{\beta}\left(t^{\prime}\right) d t^{\prime}+\phi_{z}^{\prime}\right)
\end{aligned}
$$

Where $y_{\beta}(t)=A_{y}^{\prime} / \gamma(t)^{1 / 4}$ and $z_{\beta}(t)=A_{z}^{\prime} / \gamma(t)^{1 / 4}$ time dependent amplitudes, with $\left(A_{y}^{\prime}, \phi_{y}^{\prime}\right)$ and $\left(A_{z}^{\prime}, \phi_{z}^{\prime}\right)$ constants to be determined by initial conditions. The betatron oscillation amplitude is equal then to $r_{\beta}=\sqrt{y_{\beta}(t)^{2}+z_{\beta}(t)^{2}}$. The transverse betatron oscillations are still sinusoidal but are time dependent with $\gamma(t)$.

\section{II.1.3.b Radiation properties}

Many properties such betatron emission divergence, spectrum, radiation energy spread and number of emitted photons depend on the nature of oscillation the electrons are performing inside the ion channel. This is why the strength parameter $K$ (dimensionless parameter) separating two electron oscillation regimes is introduced. The first one, is the undulator regime and corresponds to when the electron oscillates transversally and radiates in the same direction all the time during its motion. This occurs when the opening angle of emission $\Delta \theta=1 / \gamma$ is larger than the maximum angle of the trajectory $\Psi$ (see Fig.II.11.Top). In the wiggler regime, different sections of the trajectory radiate in different angles. Also, what makes it different from the undulator regime is that the cone angle of emission is much smaller than the trajectory angle $\Delta \theta=1 / \gamma \ll \Psi$. The 
wiggler regime is shown in Fig.II.11.Bottom. According to [Corde et al., 2013], the expression of the strength parameter $K$ is given by

$$
\begin{aligned}
& K=r_{\beta} k_{p} \sqrt{\gamma / 2} \\
& K\left[\mathrm{~cm}^{-3}\right]=1.33 \times 10^{-10} \sqrt{\gamma n_{e}\left[\mathrm{~cm}^{-3}\right]} r_{\beta}[\mu \mathrm{m}]
\end{aligned}
$$

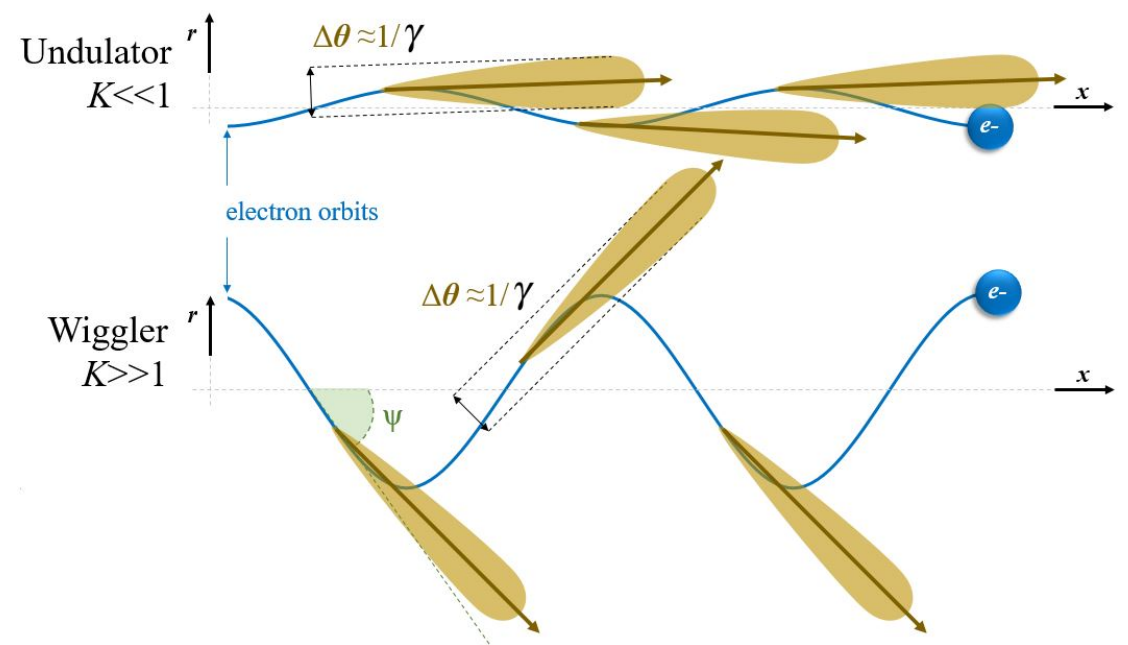

FIGURE II.11: Schematic representation of the undulator and wiggler limits. Radiation emission is shown in brown. $\Delta \theta$ is the opening angle of the cone emission and $\Psi$ is the trajectory angle. Inspired from [Corde et al., 2013].

We can predict the number of photons $N_{\gamma}$ emitted per period and per electron as a function of the parameter $K$ applying the following formula in practical units

$$
\begin{array}{cc}
N_{\gamma}=1.53 \times 10^{-2} K^{2} & \text { for } K<1 \\
N_{\gamma}=3.31 \times 10^{-2} K^{2} & \text { for } K \gg 1
\end{array}
$$

The emission spectra in the undulator regime, where $K \ll 1$ (electron is oscillating as a dipole in average rest frame) consists of a single peak centered on a frequency $\omega$ depending on the angle of observation. Concerning the wiggler case $(K \gg 1)$, the emission is no longer a peak but becomes a synchrotron like spectrum. According to [Corde et al., 2013], the radiated energy per unit frequency and per unit time is given by

$$
\frac{d P}{d \omega}=\frac{P_{\gamma}}{\omega_{c}} S\left(\omega / \omega_{c}\right)
$$

Where $S\left(\omega / \omega_{c}\right)$ is plotted in Fig.II.12 and is given by

$$
S\left(\omega / \omega_{c}\right)=\frac{9 \sqrt{3}}{8 \pi}\left(\omega / \omega_{c}\right) \int_{\omega / \omega_{c}}^{\infty} \mathrm{K}_{5 / 3}(\xi) d \xi
$$


with $\mathrm{K}_{5 / 3}$ ( $\neq K$ the strength factor) the modified Bessel function of the second kind of order $5 / 3$ and

$$
P_{\gamma}=\frac{2 e^{2} \omega_{c}^{2}}{27 \pi \varepsilon_{0} c \gamma^{2}}
$$

$\omega_{c}$ defines the critical synchrotron frequency and is given by

$$
\omega_{c}=\frac{3}{2} \gamma^{3} \frac{c}{\rho}
$$

Here $\rho$ is the radius of curvature of the electron trajectory. For a sinusoidal trajectory the critical frequency expression of Eq.II.32 becomes

$$
\omega_{c}=\frac{3}{2} K \gamma^{2} \frac{2 \pi c}{\lambda_{B}}
$$

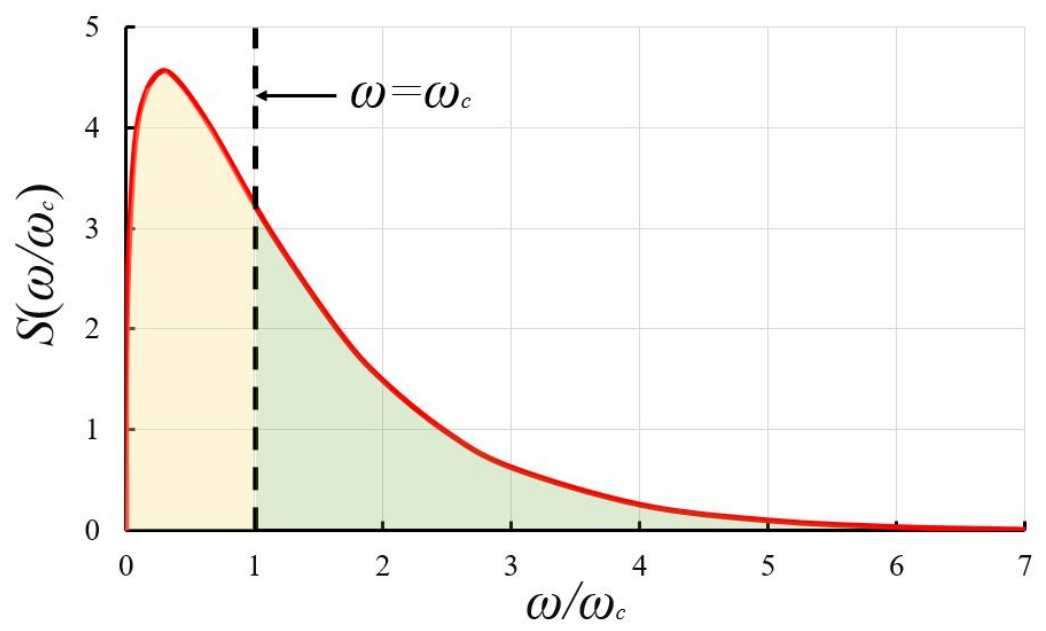

FIGURE II.12: Plot of a synchrotron like spectral emission function $S\left(\omega / \omega_{c}\right)$.

We can see from Fig.II.12 that the critical frequency is separating two regions of the synchrotron spectrum. Basically, it determines the energy at which the integrated spectral density is equal at both sides of the spectrum, which is the case of the regions in green and in orange colour.

\section{II.2 X-ray transport optics and measurement}

\section{II.2.1 X-ray matter interaction theory}

Let's consider a planar electromagnetic wave propagating in a given medium. The electric field of such radiation can be written as

$$
E=E_{0} e^{i(k r-\omega t)}
$$


Where $E_{0}$ is the amplitude of the electric field, $k$ is the wave number vector and $\omega$ is the angular frequency of the wave. Defining $\lambda$ as the radiation wavelength, where $\lambda=2 \pi / k=2 \pi c / n \omega$, we can write

$$
k=\frac{n \omega}{c}
$$

Where $c$ and $n$ are respectively the speed of light and the refractive index of the media. If the latter is irradiated by an electromagnetic wave in the x-ray domain, the index of refraction becomes complex and is given by

$$
n^{*}=1-\delta-i \beta
$$

Where $\delta$ is the refractive index decrement responsible of the phase shift and $\beta$ is the absorption coefficient term. $\delta$ and $\beta$ are written according to [Henke et al., 1993, Wu and Liu, 2003] as

$$
\begin{aligned}
& \delta=\left(\frac{r_{e} \lambda^{2}}{2 \pi}\right) \sum_{k} N_{k}\left(Z_{k}+f_{1 k}(\lambda)\right) \\
& \beta=\left(\frac{r_{e} \lambda^{2}}{2 \pi}\right) \sum_{k} N_{k}\left(Z_{k}+f_{2 k}(\lambda)\right)
\end{aligned}
$$

Here $r_{e}$ is the classical electron radius $\left(e^{2} / m_{e} c^{2}=2.8179 .10^{-15} m\right), N_{k}$ is the atomic density of the element $\mathrm{k}$ (number of atoms $k$ per unit of volume) and $f_{k}$ is the atomic scattering factor of the element $k$ where $f_{k}=f_{1 k}+i f_{2 k}$. Tabulated values of $f$ in function of the atomic number and the radiation wavelength can be found in [Henke et al., 1993]. Additional information are available in the CXRO (Centre for X-ray Optics) website [CXRO, 2019] where the X-ray database of nearly all material could be found. According to [Wu and Liu, 2003], $\delta$ can also be written in practical formulas and is given by

$$
\delta \simeq 4.49 \times 10^{-16} \lambda\left[\mathrm{m}^{2}\right] n_{e}\left[\mathrm{~m}^{-3}\right]
$$

Where $n_{e}$ is the electronic density. This formula is not valid for $\mathrm{x}$-ray values near the absorption edges. Fig.II.13 is showing an example of numerical calculations for the functions $\mathrm{f}_{1}, \mathrm{f}_{2}, \beta$ and $\delta$ in function of the incident photon energy for Silicon obtained with [XOP, 2019] (X-ray Oriented Program).

By inserting the wave vector $k$ definition (Eq.II.35) in the electric field equation (Eq.II.34), we get an explicit electric field expression in which three main parts can be clearly highlighted. The new electric field expression for a electromagnetic $\mathrm{x}$-ray wave propagating in a medium is written

$$
\boldsymbol{E}=\underbrace{\boldsymbol{E}_{0} e^{i \omega(r / c-t)}}_{\text {Free propagation }} \underbrace{e^{-i(\delta 2 \pi / \lambda) r}}_{\text {Phase shift }} \underbrace{e^{-(\beta 2 \pi / \lambda) r}}_{\text {Absorption }}
$$

The first part of the expression represents the propagation of an electromagnetic wave in vacuum without any attenuation. The second part stands for the phase shifting property of the wave due to passing through matter. The last term represents the attenuation of the wave amplitude which is due to absorption.

Let's consider now, an interaction between a free propagating x-ray beam (in vacuum) and a perfectly flat mirror. We assume that the beam is arriving at normal incidence $\left(0^{\circ}\right)$ to the mirror's 

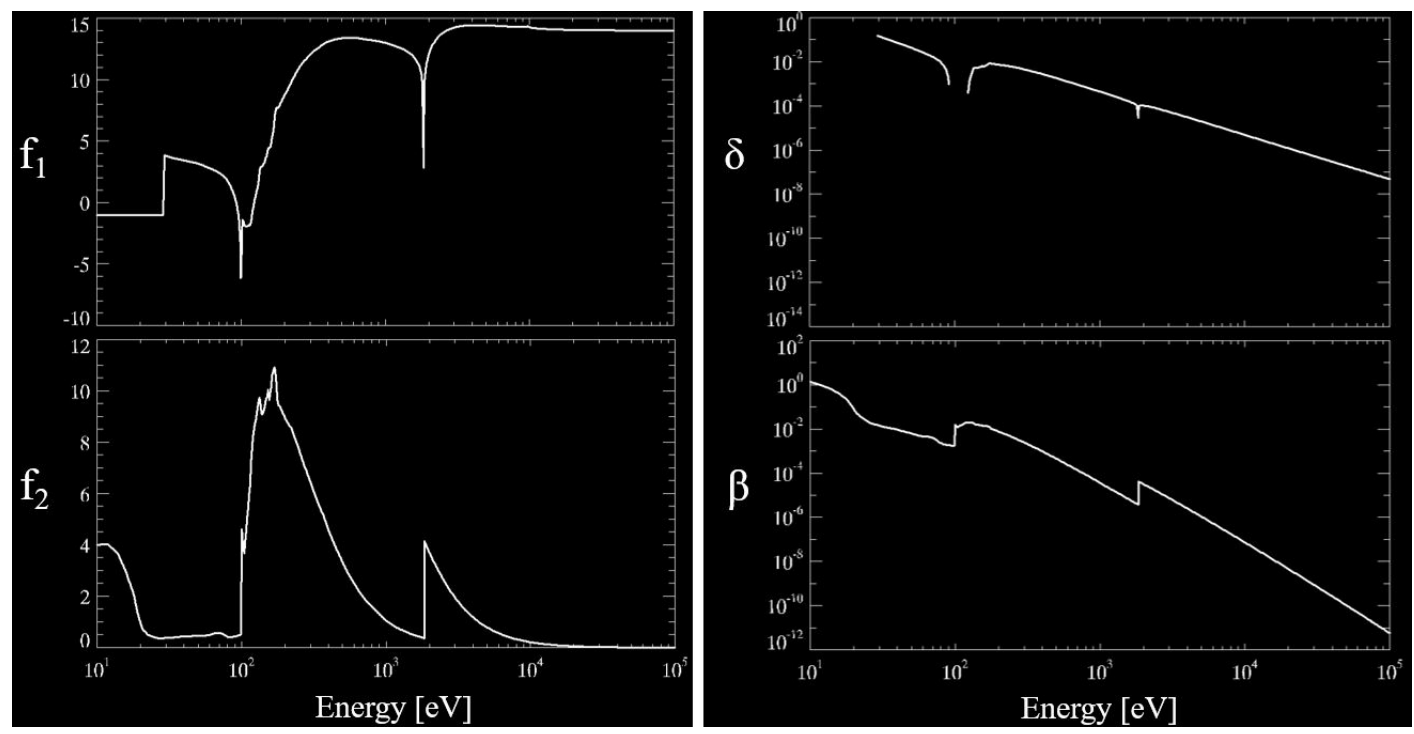

FIGURE II.13: XOP simulations for the anomalous scattering functions $\mathrm{f}_{1}, \mathrm{f}_{2}$, absorption coefficient $\beta$ and phase shift decrement $\delta$ in function of the incident photon energy for silicon.

surface. According to [Michette, 1986], the reflectivity formula at boundary conditions is given by

$$
\left.R\right|_{0^{\circ}}=\left|\frac{1-n^{*}}{1+n^{*}}\right|^{2}=\frac{\delta^{2}+\beta^{2}}{(2-\delta)^{2}+\beta^{2}}
$$

As an example, we can calculate $\left.R\right|_{0^{\circ}}$ for an interaction between an incoming x-ray beam with a wavelength of $0.8 \mathrm{~nm}$ (corresponding to $1550 \mathrm{eV}$, the $\mathrm{K}-\alpha$ emission line of Aluminium) and a perfectly flat platinum coated mirror. The tabulated values of $\delta$ and $\beta$ at this $\mathrm{x}$-ray energy are 9.71.10 $0^{-4}$ and $2.68 .10^{-4}$ respectively, and so, $\left.R\right|_{0^{\circ}}$ is then equal to $2.54 .10^{-7}$. This value of reflectivity is very low if compared to the efficiency of other x-ray focusing techniques.

Considering the parameters defined in Fig.II.14, the general reflectivity formula is given by the Fresnel law at boundary positions between two mediums of index $\left(n_{1}\right.$ and $\left.n_{2}\right)$ by

$$
\begin{aligned}
& R_{s}=\frac{\left|n_{1} \cos \left(\theta_{i}\right)-n_{2} \cos \left(\theta_{t}\right)\right|^{2}}{\left|n_{1} \cos \left(\theta_{i}\right)+n_{2} \cos \left(\theta_{t}\right)\right|^{2}} \\
& R_{p}=\frac{\left|n_{2} \cos \left(\theta_{i}\right)-n_{1} \cos \left(\theta_{t}\right)\right|^{2}}{\left|n_{2} \cos \left(\theta_{i}\right)+n_{1} \cos \left(\theta_{t}\right)\right|^{2}}
\end{aligned}
$$

Where $s$ and $p$ stand for parallel and perpendicular radiation polarisations relatively to the reflecting surface plane. $\theta_{i}$ is the angle of incidence (for an incident beam from medium 1 to medium 2 ) and $\theta_{t}$ is the angle of the transmitted beam (in medium 2). We consider here the normal axis to the reflecting surface plane as a reference, at which $\theta_{i}=\theta_{t}=0$. 


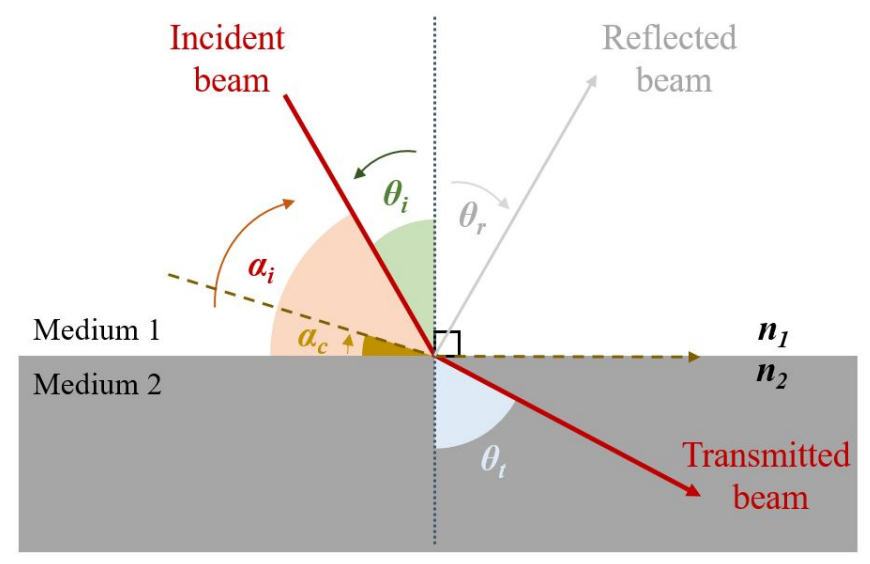

FIGURE II.14: Parameter definition for an incident, reflected and transmitted beams at boundary positions.

The expression of the unpolarized beam reflectivity formula is given by

$$
R=\frac{R_{s}+R_{p}}{2}
$$

We can calculate numerically the reflectivity functions $R_{s}, R_{p}$ and $R$ for the conditions mentioned above using XOP for incident angles going from $0^{\circ}$ up to $90^{\circ}$. The results of the calculations are plotted in Fig.II.15

We can see in Fig.II.15 the evolution of reflectivity as a function of the angle of incidence $\theta_{i}$, from which we note an increase of $R$ for ultra-wide incidence angles. Indeed, at normal angles of incidence, most media have a very low reflectivity (many orders of magnitude less than unity), but this effect can be overcome if working at grazing incidence angles $\theta_{i} \sim 90^{\circ}$ (see Fig.II.15). Let us then define $\alpha_{i}$ as the grazing incidence angle i.e. the complementary angle of $\theta_{i}$, where $\alpha_{i}=\pi / 2-\theta_{i}$. Thus, we can conclude that $R$ increases for large $\theta_{i}$ and so, for small $\alpha_{i}$. For exactly this reason many total reflection based $\mathrm{x}$-ray diagnostics operate at grazing incidence angles. The Kirkpatrick-Baez microscopes is one of them and is described in detail in the next chapter.

There exists a critical grazing incidence angle $\alpha_{c}$, satisfying the total external reflection conditions and it can be obtained from Fresnel law at $\theta_{t}=90^{\circ}$ from normal. We have then

$$
\cos \left(\alpha_{c}\right)=\frac{n_{2}}{n_{1}}=\frac{1-\delta_{2}}{1-\delta_{1}}
$$

In case of grazing incidence angles such as $\left(\alpha_{i}=\alpha_{c}\right) \rightarrow 0$, we can use the small angles approximation relation, $\cos \left(\alpha_{i}\right) \approx 1+\left(\alpha_{i} / 2\right)^{2}$. For a free propagating $\mathrm{x}$-ray wave in vacuum $\left(\delta_{1}=\beta_{1}=0\right)$ interacting at grazing angle with a mirror $\left(\delta_{2} \neq \beta_{2} \neq 0\right)$, the critical angle can be written

$$
\alpha_{c}=\sqrt{2 \delta_{2}}
$$

The critical wavelength $\lambda_{c}$ will be then given by

$$
\lambda_{c}=\frac{2 \pi c \alpha_{c}}{e} \sqrt{\frac{\varepsilon_{0} m_{e}}{n_{e}}}
$$



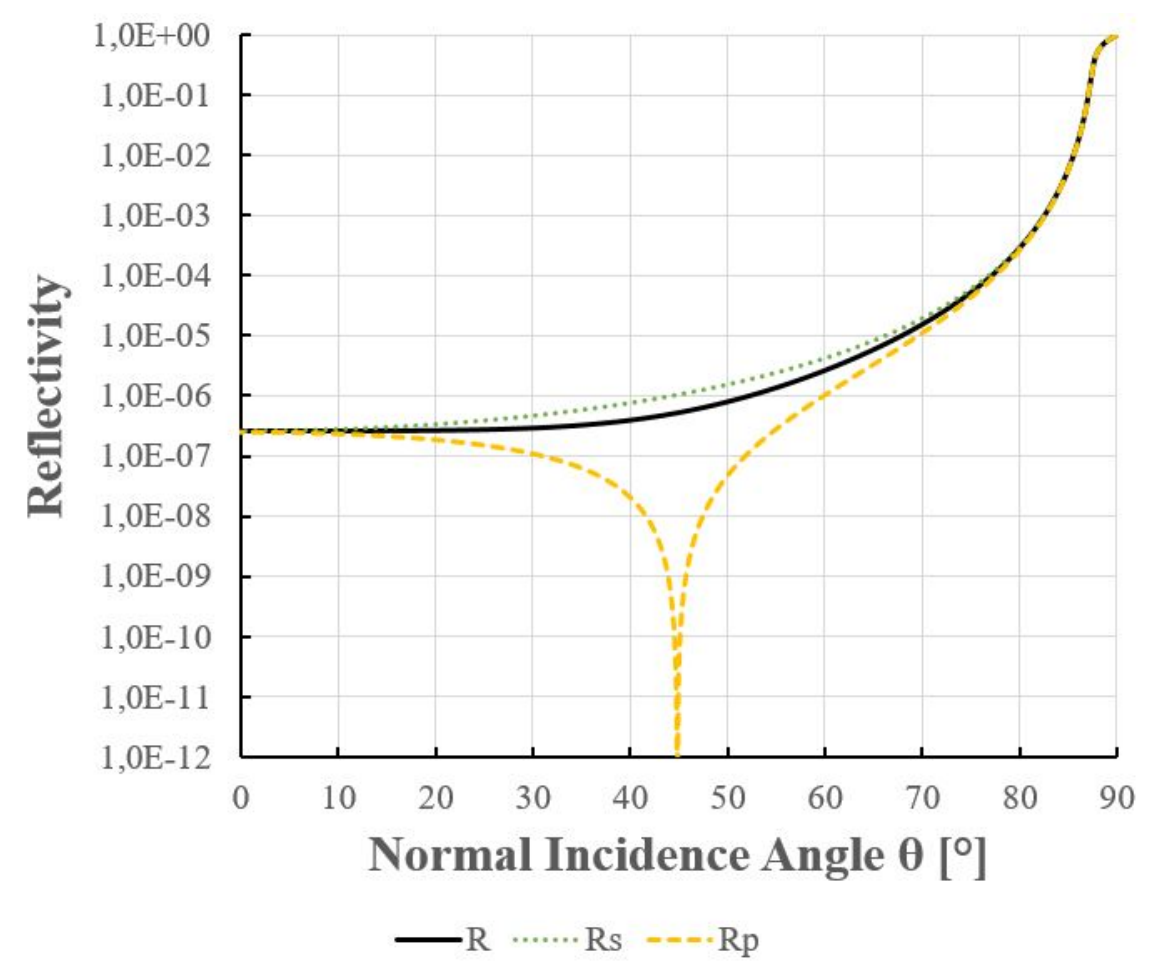

FIGURE II.15: Calculated $R_{s}, R_{p}$ and $R$ for an incident x-ray beam of 1550 $\mathrm{eV}$ corresponding to the $\mathrm{K} \alpha$ emission line of aluminium. We note that the Brewster angle is situated at $45^{\circ}$ where $R_{p}$ drops dramatically. At this angle of incidence we have $R=R_{s} / 2$, the parallel component $R_{p}$ is completely absorbed/transmitted.

From the expression of $\lambda_{c}$, we note that only the radiation of $\lambda>\lambda_{c}$ is totally reflected. The reflectivity dependence on the incident x-ray energy is also clearly highlighted in Eq.I.46. We conclude that, for fixed angles of incidence, higher photon energy $x$-rays are in general attenuated more strongly. The effect of both the grazing incidence angle and the incident $\mathrm{x}$-ray photon energy are plotted using XOP in Fig.II.16 for a platinum mirror. A range of photon energies up to $10 \mathrm{keV}$ and grazing incidence angles up to $60 \mathrm{mrad}$ are plotted. Choosing a platinum mirror for the calculation as an example hasn't been done randomly and the reason behind it will be discovered in the next chapter.

Last, but not least, it is noteworthy to mention that in reality, the mirrors in general, are not as perfect as in the calculations of reflectivity shown in Fig.II.16 (where the root-mean-square surface height was supposed to be equal to zero). Indeed, mirrors scatter light too and so, a portion of the beam gets lost during the x-ray transport. Thus, it is necessary to introduce the roughness parameter, defined as the measure of the rms height of an optical surface and we will see how it causes significant reflectivity degradation for single coated metallic mirrors. According to [Bennett and Porteus, 1961], roughness was first related to the specular reflection at normal incidence angles then was extented to refraction and to non-normal incidence. The expression relating the roughness to the total integrated scatter, TIS, can be fund in [Harvey et al., 2012] 


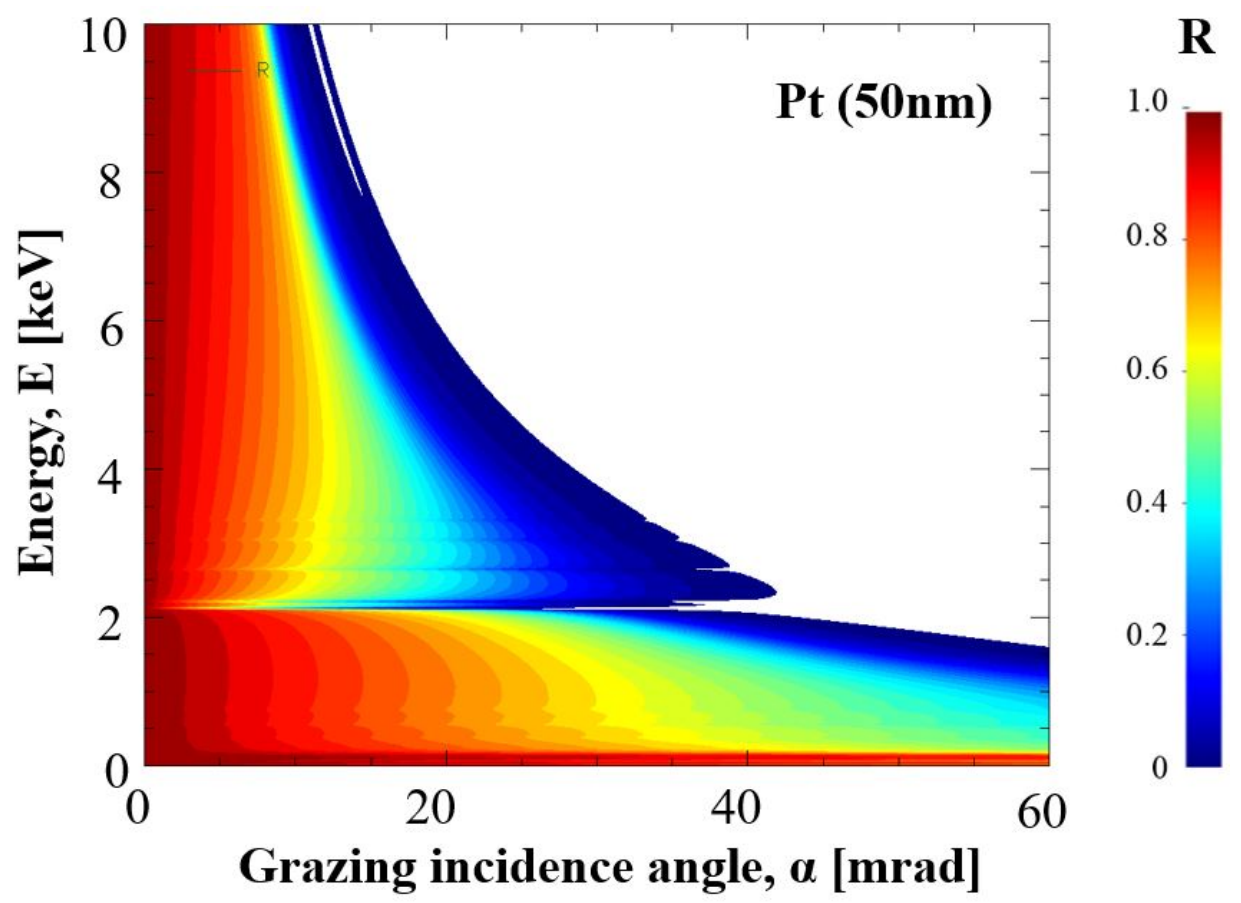

FIGURE II.16: Calculated reflectivity map of a layer of 50nm thickness as a function of grazing angle of incidence and $\mathrm{x}$-ray photon energy.

starting from the definition of TIS

$$
\mathrm{TIS}=\frac{\text { Diffuse reflectance }}{\text { Total reflectance }}=\frac{\text { Diffuse reflectance }}{\text { Specular reflectance }+ \text { Diffuse reflectance }}=\frac{R_{\mathrm{d}}}{R_{\mathrm{spc}}+R_{\mathrm{d}}}
$$

Where $R_{\mathrm{d}}$ and $R_{\mathrm{spc}}$ stand for diffuse reflectance and specular reflectance. Definig $R_{\mathrm{T}}$ as the total reflectance where $R_{\mathrm{T}}=R_{\mathrm{spc}}+R_{\mathrm{d}}$ we can write

$$
R_{\mathrm{spc}}=R_{\mathrm{T}} \exp \left[-\left(4 \pi \cos \theta_{i} \sigma / \lambda\right)^{2}\right]
$$

Where $\theta_{i}$ is the normal incidence angle, $\lambda$ is the incident photon wavelength and $\sigma$ is the rms surface roughness. Replacing $R_{\mathrm{d}}$ in Eq.II. 47 we find the following expression of TIS as a function of $\sigma$

$$
\mathrm{TIS}=\frac{R_{\mathrm{T}}-R_{\mathrm{spc}}}{R_{\mathrm{T}}}=1-\exp \left[-\left(4 \pi \cos \theta_{i} \sigma / \lambda\right)^{2}\right]
$$

Few conclusions can be made from Eq.II.49. First, we can see that scattering is more important for shorter wavelengths than for longer ones. We can also observe that the scattering increases for higher rms surface roughness $\sigma$ values. We can also see, from Eq.II.49, that incident photons at normal angle of incidence scatter more than if they were incident at grazing angles.

Finally, we have used XOP program to calculate the variations of reflectivity as a function of incident photon energies for different materials (usually used to coat x-ray optics), for a fixed grazing angle of incidence and for different roughness values $\sigma$ (See Fig.II.17).

According to Fig.II.17, we can see clearly how the reflectivity drops as a function of the roughness. This effect gets even more important for high energy incident photons as expected from 
the formula in Eq.II.49. Thus, the higher is the roughness, the more photons scatter so, the lower the reflectivity gets. This non-negligible parameter needs to be taken into account for reflectivity measurements especially for precise estimation operations.
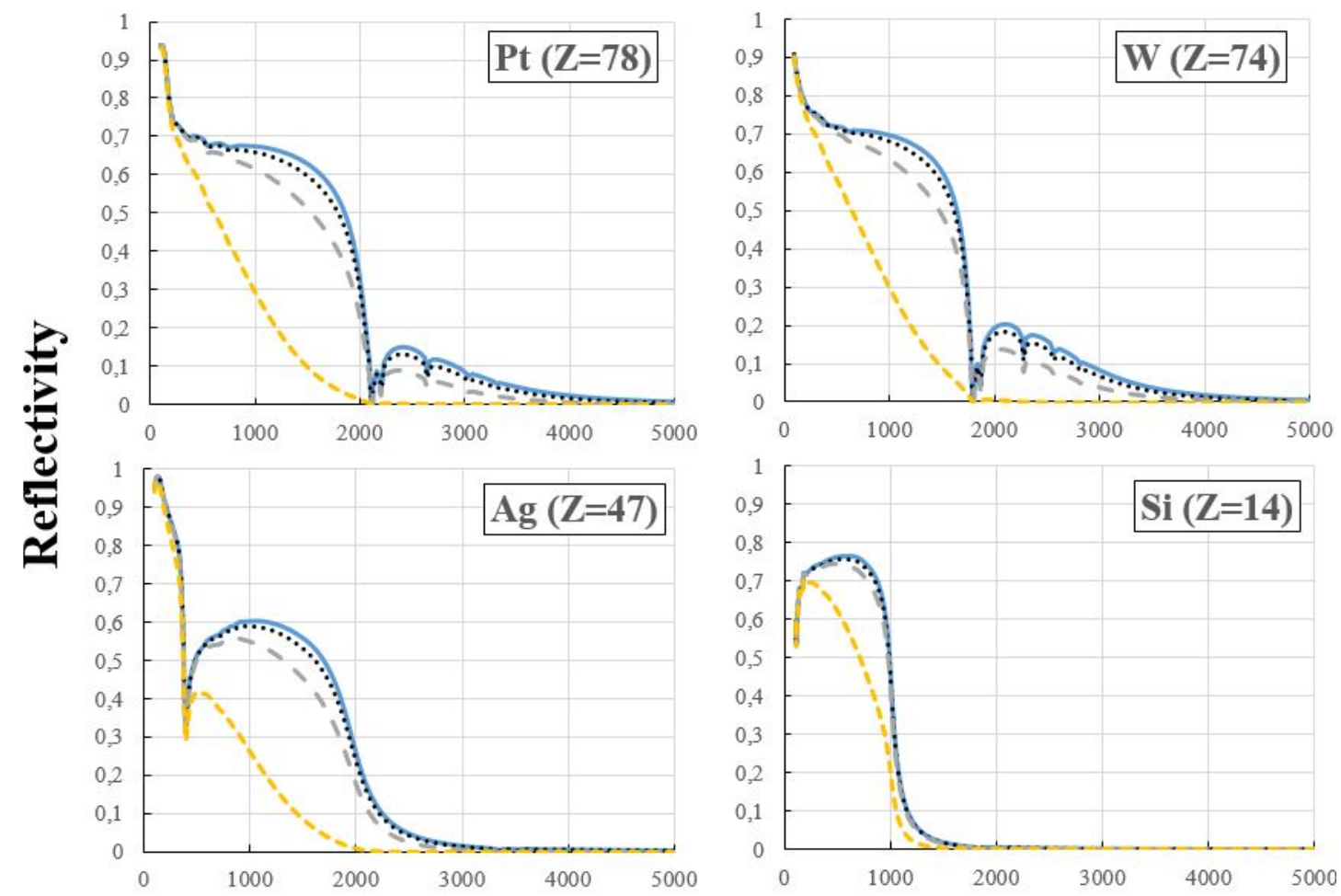

Incident Photon Energy in [eV]

$$
\begin{array}{ll}
\boldsymbol{\sigma}=0 \AA & \boldsymbol{\sigma}=10 \AA--- \\
\boldsymbol{\sigma}=5 \AA \ldots \ldots \ldots . . & \boldsymbol{\sigma}=30 \AA
\end{array}
$$

FIGURE II.17: Calculated reflectivty for different coating materials (of 50nm thickness), as a function of incident photon energy for a fixed grazing incidence angle $\alpha=30 \mathrm{mrad}$.

\section{II.2.2 Bragg crystals}

Bragg crystals have been used since the beginning of the $20^{\text {th }}$ century for mainly two purposes, imaging and spectroscopy. These crystals have a specific inter-planar spacing which optimises the constructive interferences of the reflected x-ray radiation at a specific angle called, Bragg angle $\theta_{B}$. Only the radiation of wavelength $\lambda_{B}$ satisfying the so-called, Bragg law, as shown in Fig.II.18 are reflected. This latter is given by Eq.II.50 as

$$
n \lambda_{B}=2 d \sin \left(\theta_{B}\right)
$$

where $n$ is the diffraction order and $d$ is the periodic distance between the crystal planes. Fig.II.18 shows a pair of incident beams on a perfect crystal satisfying the Bragg condition. This one is 


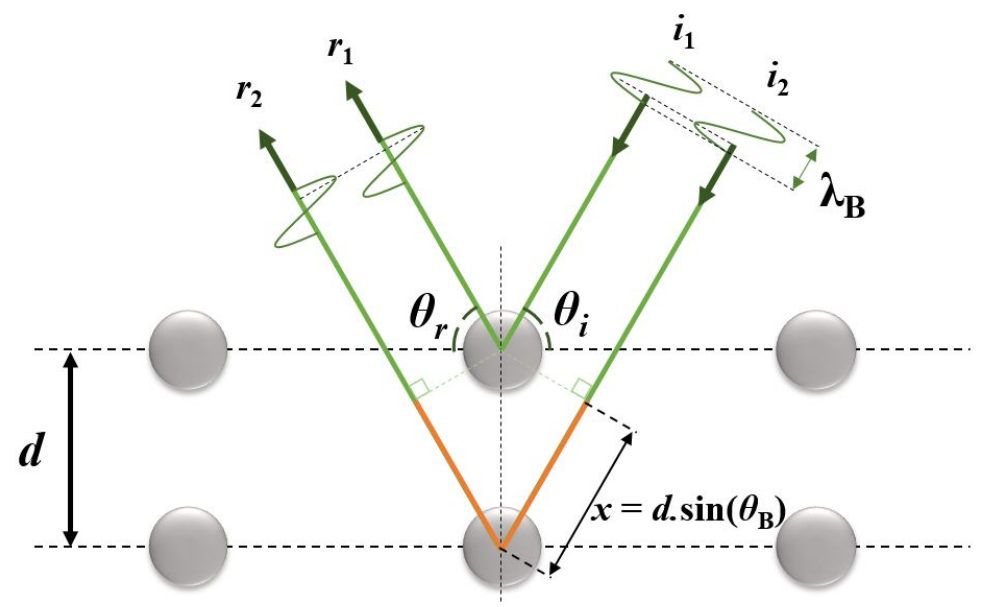

FIGURE II.18: Bragg reflection principle. In green, an example of two incident beams $i_{1}$ and $i_{2}$, reflected at the Bragg angle $\theta_{\mathrm{B}}$. Here $\theta_{\mathrm{B}}=\theta_{i}=\theta_{r}$. The path difference and the atoms are shown in orange and grey colours respectively.

fulfilled when the path difference between the two beams (in orange colour) is a multiple of the incident beam wavelength such that

$$
2 x=n \lambda_{B}
$$

This condition leads to the Bragg law, Eq.II.50. It is noteworthy to mention that Bragg formula needs to be corrected in the x-ray range, mainly for the phase shift factor and is given according to [Antonelli et al., 2015] as

$$
n \lambda_{B}\left(1+\frac{\delta d^{2}}{4 \lambda_{B}^{2}}\right)=2 d \sin \left(\theta_{B}\right)
$$

This correction is usually negligible for some crystals since $\delta$ is in the order of $10^{-6}$. It is important to note that this relation is also valid for multilayer coatings [Roling et al., 2014]. These consist of a periodic alternation of high and low $\mathrm{Z}$ elements to optimize the $\mathrm{x}$-ray reflection. More details about this issue are given further in this thesis.

\section{II.2.2.a Bragg crystals for X-ray spectroscopy}

\section{II.2.2.a.1 Flat Bragg crystals}

\section{Perfect Bragg crystals}

With the property of optimizing the reflectivity of distinct energies at precise angles, makes Bragg crystals very useful in laser-plasma experiments for looking at specific plasma emission lines. This ability of energy filtering permits the full characterisation of the emission line of interest with a reasonable signal to noise ratio. The reflectance curve of Bragg crystals versus incidence angle, also called the rocking curve, can be calculated using ray-tracing programs. Fig.II.19 (bottom) shows an example of the reflectance obtained numerically with XOP of an $\alpha$-quartz crystal for an incident $\mathrm{x}$-ray beam of $\mathrm{Cu}-\mathrm{k} \alpha(8.05 \mathrm{keV})$ as a function of the grazing incidence angle around the Bragg angle $\theta_{B}$. The crystal reflectance $r$ can also be calculated using 
simple models such as the Darwin curve for planar crystals [Antonelli et al., 2015]. This one is given by

$$
r(x)= \begin{cases}R_{\max } & \text { if }\left|\frac{x}{\Delta \theta}\right| \leq 1 \\ \frac{R_{\max }}{\left(|x / \Delta \theta|^{2}+\sqrt{|x / \Delta \theta|^{2}-1}\right)^{2}} & \text { if }\left|\frac{x}{\Delta \theta}\right|>1\end{cases}
$$

Here, $\Delta \theta$ is the half width of the curve plateau where $r=R_{\max }$ and $x$ is $\theta_{i}-\theta_{B}\left(\lambda_{B}\right)$. We note that the expression for $\Delta \theta$ can be complex to calculate since its depending on many crystal parameters such as the structure factor, polarizability, etc ... More details concerning this issue can be fund in [Antonelli et al., 2015].

Generally, when using Bragg crystals in experiments it is necessary to calculate the spectral distribution function $E_{v}=f(y)$ at the x-ray detector (Image Plates, X-ray camera...) plane as shown in Fig.II.19.
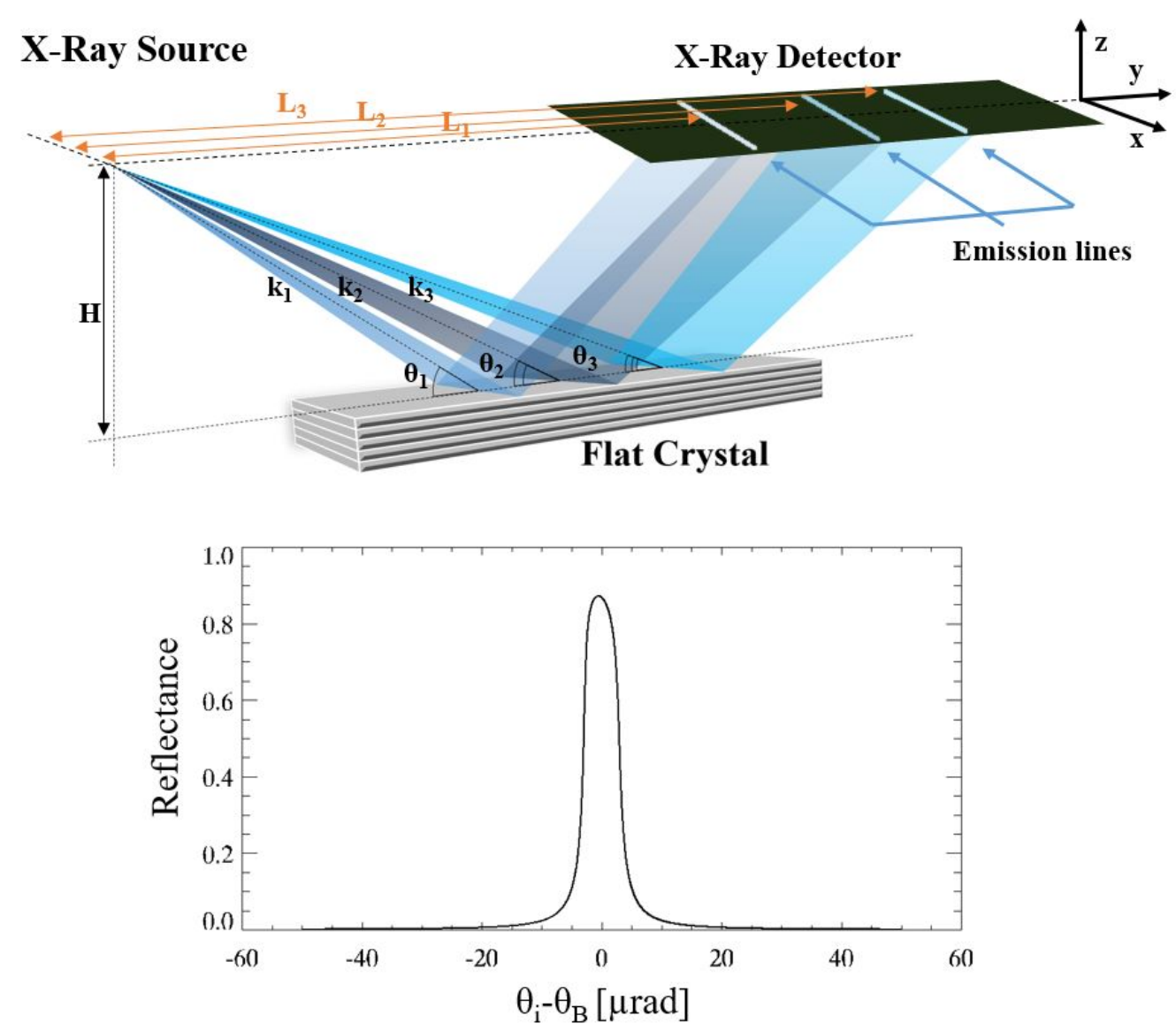

FIGURE II.19: Schematic diagram of a Bragg crystal reflecting the radiation emitted from a broadband point x-ray source (Top). Reflectance curve of an $\alpha$-quartz crystal (111), of $1 \mathrm{~cm}$ thickness as a function of the grazing incidence angle $\theta_{i}$ around the specific Bragg angle $\theta_{B}$ for incident radiation of energy 8.05 $\mathrm{keV}$. The calculation takes into account the photo electric effect and also both Compton and Rayleigh scattering. 
Using the notations shown in Fig.II.19 we can write

$$
\sin \left(\theta_{i}\right)=\frac{\mathrm{H}}{\mathrm{k}_{i}}=\frac{\mathrm{H}}{\sqrt{\mathrm{H}^{2}+\mathrm{L}_{i}^{2} / 4}}
$$

Combining both, Eq.II.54 and Eq.II.50, we can write

$$
n \lambda=n \frac{h c}{E_{v}}=2 d \frac{\mathrm{H}}{\sqrt{\mathrm{H}^{2}+\mathrm{L}_{i}^{2} / 4}}
$$

So, for $\mathrm{L}_{i} \equiv y$ we can write

$$
E_{v}=n \frac{h c}{2 d} \frac{\sqrt{\mathrm{H}^{2}+\mathrm{y}^{2} / 4}}{\mathrm{H}}=n \frac{h c}{2 d} \sqrt{1+\frac{y^{2}}{4 \mathrm{H}^{2}}}
$$

From Eq.II.56, one can realize that $\mathrm{E}_{v}$ becomes approximately linear for long distances $(\mathrm{y}>>\mathrm{H})$ and is given by

$$
E_{v} \simeq n \frac{h c}{2 d} y
$$

On the other hand, we can also calculate the spectral resolution of the detector by differentiating Eq.II.56

$$
\Delta E_{v}=n \frac{h c}{2 d} \frac{y}{4}\left(\mathrm{H}^{2}+y^{2} / 4\right)^{-\frac{1}{2}} \Delta y
$$

We can then write

$$
\frac{\Delta E_{v}}{E_{v}}=\frac{y}{4 \mathrm{H}^{2}+y^{2}} \Delta y
$$

It is noteworthy to mention that in this case the emission lines are detected in the (xy) plane. It is then clear that the signal is stretched along the $\mathrm{x}$ direction (as seen in Fig.II.19.Top). Due to this fact, and to the divergent properties of laser driven x-ray sources, the signal to noise ratio decreases with distance from the source. On the top of that, as shown in Fig.II.19 (bottom), the reflectance curve is "sharp" (the curve is more a delta function than a Gaussian) which implies a high resolution in the spectral detection.

\section{Imperfect Bragg crystals: HOPG}

Another alternative to perfect Bragg crystals is to use a mosaic crystal structure. These are ones made of a distribution of microscopic perfect crystals oriented in the crystal plane direction. One of the most commonly used mosaics in spectroscopy (for the Laser-Plasma community) is the HOPG [Legall et al., 2006]. The latter stands for Highly Oriented Pyrolitic Graphite and can be found in both states, mosaic and perfect crystals. The most important difference between these states is the reflectance distribution around the Bragg angle as seen in Fig.II.20. We can also see clearly from Fig.II.20 that less peak intensity is reflected but more integrated reflectance is achieved. This artificial broadening of the emission lines increases dramatically the signal to the detriment of the resolution which of course is reduced. 


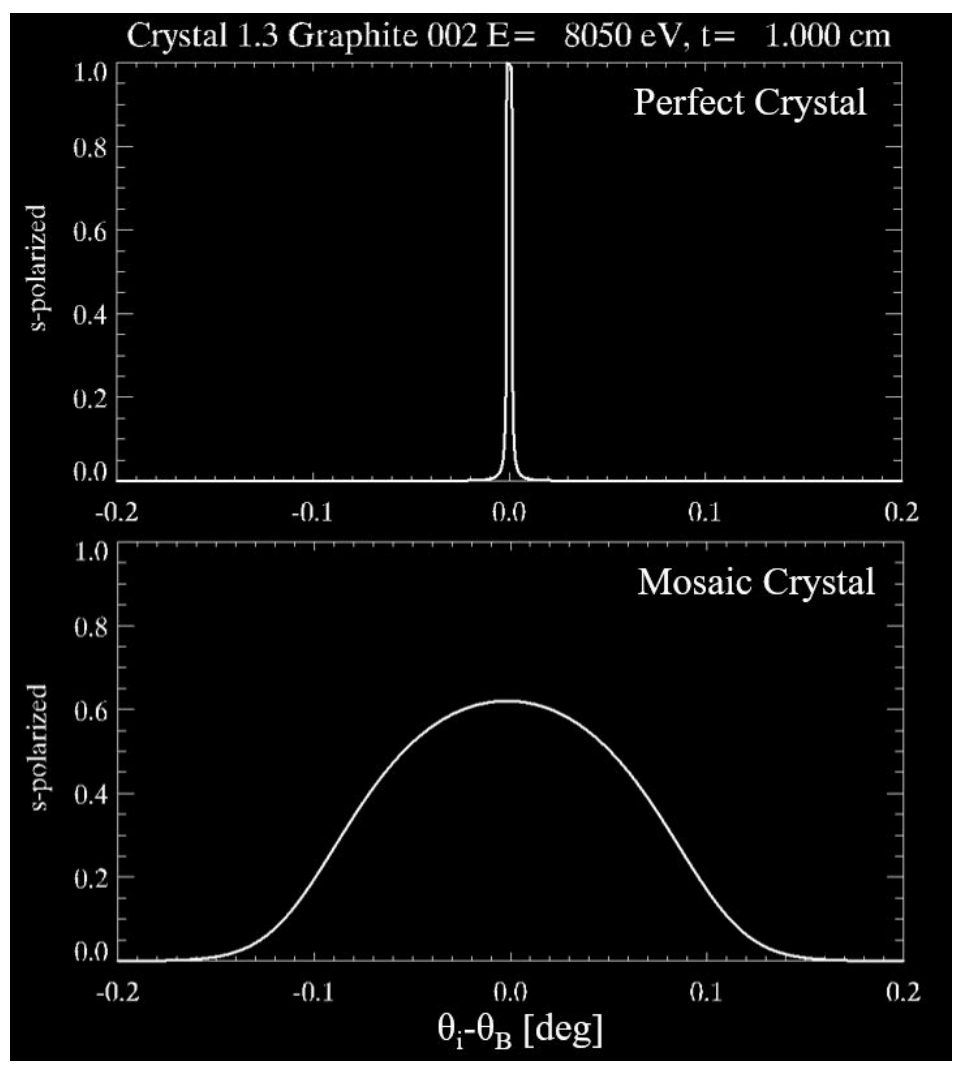

FIGURE II.20: XOP Simulated reflectance curve around the Bragg peak for the planes (002) of Graphite in two states: Perfect crystal state (top) and Mosaic state (bottom).

\section{II.2.2.a.2 Curved Bragg crystals}

Another possibility to increase the signal on the x-ray detectors is to use curved Bragg crystals as shown in Fig.II.21. Focusing the x-ray signal (emission lines) into a small spot optimises the signal to noise ratio. To avoid astigmatism, it is preferable to place the $\mathrm{x}$-ray detector at a distance equal to the Rowland circle of crystal. We note that HOPG spectrometers can also be manufactured in cylindrical (or even in conical) configuration.

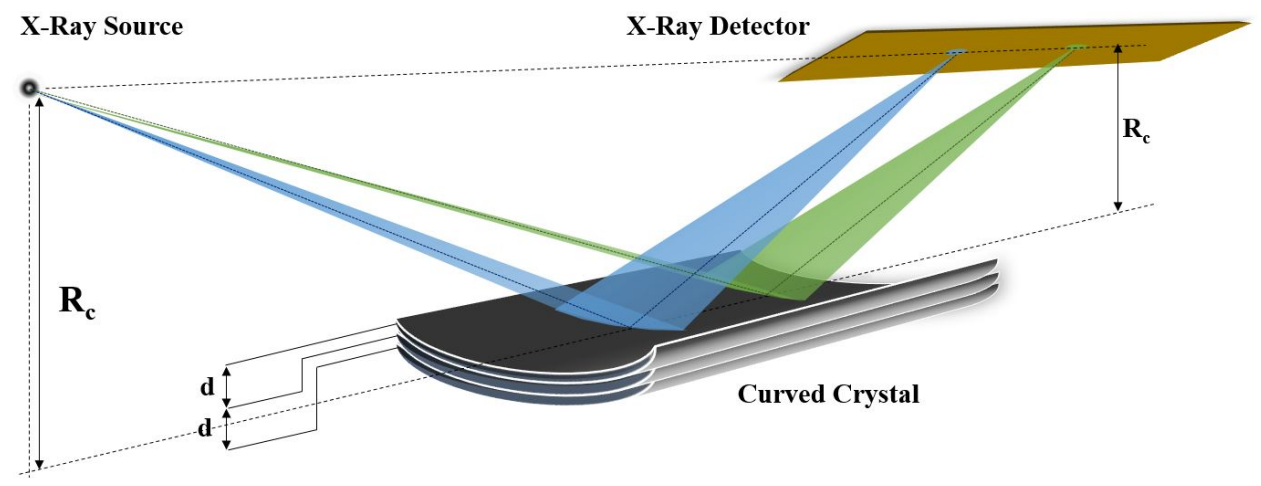

FIGURE II.21: Curved (cylindrical) crystal spectroscopy scheme. 


\section{II.2.2.a.3 Multilayer Bragg crystals}

As we previously introduced, multilayer coatings are the ultimate alternative for optimising the reflectivity. The working principle of the latter is also based on the Bragg law. By controlling the periodical alternation of layers of high reflectance and high transmission materials we are able to create high reflectivity mirrors in the $\mathrm{x}$-ray range operating at wider angles for fixed energies. Some of the most popular multilayer coatings used in the soft $\mathrm{x}$-ray range are $\left[\mathrm{W} / \mathrm{B}_{4} \mathrm{C}\right],[\mathrm{Pt} / \mathrm{C}]$, [W/C], $\left[\mathrm{Mo} / \mathrm{B}_{4} \mathrm{C}\right]$, etc... Fig.II.22 shows an example of a reflectance map calculation (using $\mathrm{XOP}$ ) for a multilayer coating $\left[\mathrm{W} / \mathrm{B}_{4} \mathrm{C}\right]$ for grazing angles up to $100 \mathrm{mrad}$ and for incident photon energies up to $10 \mathrm{keV}$.

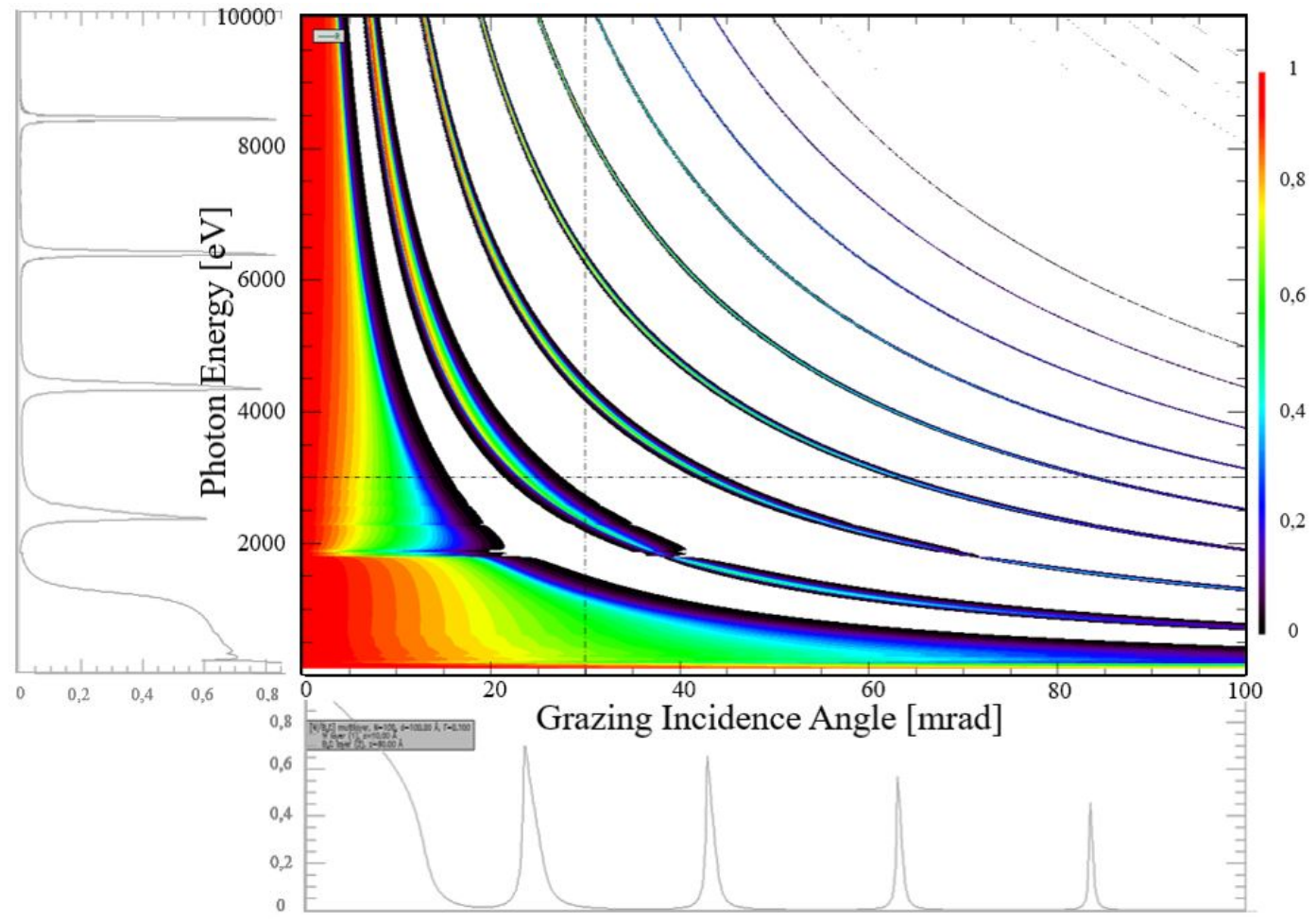

FIGURE II.22: Reflectance map for 100 layers of $\mathrm{W} / \mathrm{B}_{4} \mathrm{C}$ multilayer (centre). Each layer is $10 \mathrm{~nm}$ thick $\left[\mathrm{W}=1 \mathrm{~nm} ; \mathrm{B}_{4} \mathrm{C}=9 \mathrm{~nm}\right]$. Sample reflectance profiles for $\mathrm{R}(\theta)_{E=3 \mathrm{keV}}$ and $\mathrm{R}(\mathrm{E})_{\theta=30 \mathrm{mrad}}$ respectively at the bottom and at the left of the figure.

The four first orders of Bragg reflection are clearly highlighted in Fig.II.22. We can also see how one can reflect the same energy with approximately the same reflectance at larger grazing angles. These multilayer coatings are hard to manufacture and only few facilities can afford their cost which represents a disadvantage for their use.

\section{II.2.2.b Bragg crystals for X-ray imaging}

By merging the properties of Bragg crystals with the ones of curved (cylindrical or spherical) mirrors, one can actually carry out, spectrally resolved, imaging in the x-ray range. Usually 
spherical, curved Bragg crystals are operating at near to normal incidence as seen in Fig.II.23. Performing at these angles reduces the astigmatism and optimises the crystal aperture (totality of the crystal's aperture that is irradiated and participates to the focusing).

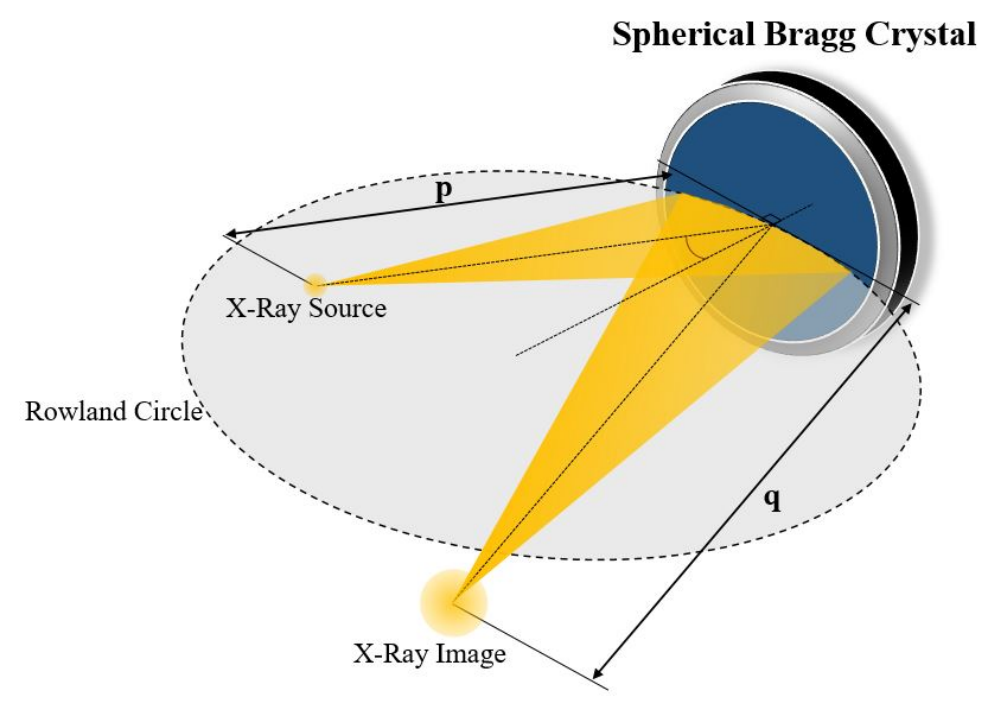

FIGURE II.23: Spherical Bragg imager scheme.

The imaging relation that governs the spherical Bragg imager (the same as for spherical mirrors) is given by

$$
\frac{1}{\mathrm{p}}+\frac{1}{\mathrm{q}}=\frac{2}{\mathrm{R}_{c r y}}
$$

Where $\mathrm{R}_{c r y}$ is the crystal's radius of curvature. The magnification factor $M$ of this imaging system is given by

$$
M=\frac{\mathrm{q}}{\mathrm{p}}
$$

Up to now we have introduced many Bragg-law-based x-ray optics where we have presented their properties, advantages and disadvantages. A very interesting alternative to all these $\mathrm{x}$-ray optics, is the Kirkpatrick-Baez microscope. This latter is presented in detail in the next chapter of this thesis. A theoretical study in addition to experimental characterisation tests and applications are presented. 


\section{Chapter III}

\section{The adjustable Kirkpatrick-Baez microscope: design, building and testing}

\section{Introduction}

We have seen from the previous chapter how challenging is to transport $\mathrm{x}$-ray radiation in the soft x-ray range (0.1-20 keV). Fortunately, thanks to the development of x-ray optics technology, many techniques are now available to operate in this x-ray domain, achieving ultra-high resolution (up to $\mathrm{nm}$ ) $\mathrm{x}$-ray focusing/imaging. From one side, Fresnel zone plates, Bragg crystals and high $\mathrm{Z}$ curved metallic mirrors are the most used $\mathrm{x}$-ray optics to transport $\mathrm{x}$-rays. In the other side, Kirkpatrick-Baez microscopes $(\mathrm{KB})$ are the most efficient in terms of $\mathrm{x}$-ray transmission. This chapter is mainly dedicated to both the theoretical and experimental characterisation of an adjustable home-made KB microscope of which I have designed, built and tested at CLPU (Spanish Center for Pulsed Lasers, see more details in the next chapter). Many singular properties of the designed KB microscope are presented in this chapter, making it a very unique tool for transporting X-rays. The working principle, theory and design of conventional KB microscopes are presented first. A detailed theoretical and experimental characterization of the home-made adjustable prototype is discussed next. Applications and results from high intensity laser-driven $\mathrm{x}$-rays obtained using the adjustable $\mathrm{KB}$ microscope are presented in the following chapter.

\section{III.1 Theory and design of the KB microscope}

\section{III.1.1 Design of the Kirkpatrick-Baez microscope}

The Kirkpatrick-Baez microscope is an imaging device working in the $\mathrm{x}$-ray range and operating at grazing angles of incidence. The first design of the KB microscope was done by P. Kirkpatrick and A. Baez in the year 1948 [Kirkpatrick and Baez, 1948]. It was introduced as an alternative to other existing x-ray imaging diagnostics such as Fresnel zone plates [Baez, 1961] and spherical Bragg crystals. Even if the latter have an important reflectivity around the Bragg peak, they still have a narrow spectral range (few eV), not adapted for broadband emission sources. Moreover, their manufacturing still represents a challenge in itself. On the other hand, Fresnel zone plates 
are known to be highly efficient in terms of x-ray transmission but these ones are highly chromatic which can also represents in itself a disadvantage. KB microscopes are characterized by a broadband spectral bandwidth (hundreds of $\mathrm{eV}$ ) and are achromatic devices. These properties make them very practical for Laser-plasma experiments. Today, we can find KB microscopes in many laser facilities around the world like the LMJ [Champeaux, 2006] in France, OMEGA [Marshall, 2017] and NIF [Pickworth, 2016] in USA.

\section{III.1.1.a General imaging relations}

The Kirkpatrick-Baez microscope consists of two cylindrically (or spherical) curved mirrors perpendicularly installed, in such way, each single mirror focuses x-rays in a single plane, creating an $\mathrm{x}$-ray image in one direction. The mirrors of the KB microscope operate separately in two perpendicular planes (one in the sagittal plane and the second in the meridional plane), providing a two dimensional image of the X-ray source. A scheme of a conventional KB microscope system is presented in Fig.III.1.

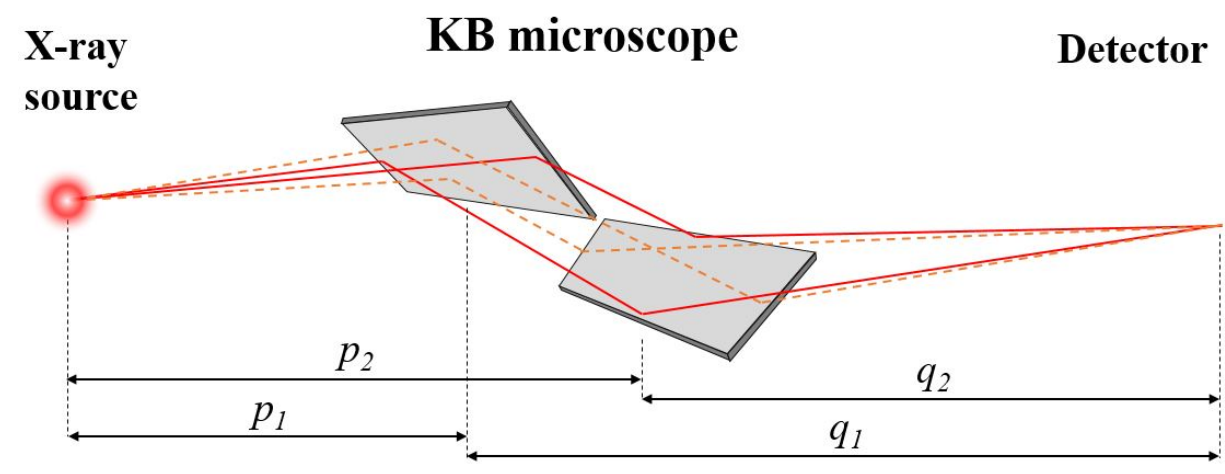

Single KB Mirror

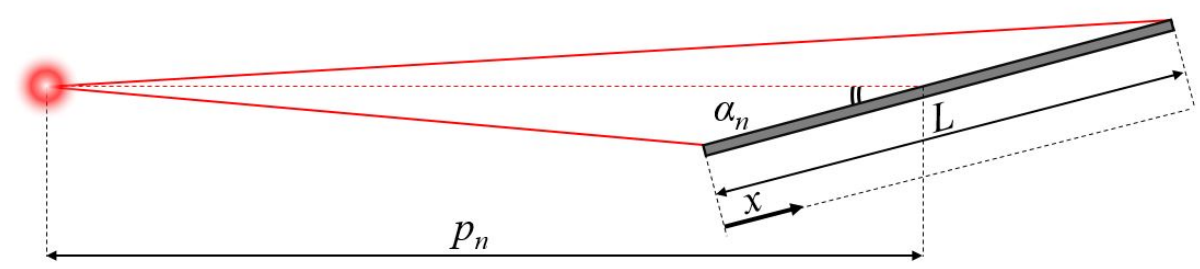

FIGURE III.1: Schematic diagram of a KB microscope system (Top). Schematic diagram of a single mirror for definition of parameters (Bottom).

We note that, for perfect 2D imaging, both generated 1D images (independently created by each mirror of the KB microscope) must be exactly at the plane of the detector. This fact implies a different radii of curvature on both mirrors. Using the defined variables in Fig.III.1, the imaging relations governing the KB microscope are given by

$$
\frac{1}{p_{n}}+\frac{1}{q_{n}}=\frac{1}{f_{n}}
$$


where

$$
f_{n}=\frac{R_{n} \sin \left(\alpha_{n}\right)}{2}
$$

Here $p_{n}$ and $q_{n}$ are the imaging distances from the x-ray source to the mirror (to the mirror center position) and from the mirror to the image position respectively. The index $n$ refers to the mirror 1 and 2 respectively. $f_{n}$ is the focal distance of the mirror, $\alpha_{n}$ is the grazing angle of incidence (defined in the previous section) and $R_{n}$ is the radius of curvature of the mirror.

It is important to notice that since we will always have $p_{1}<p_{2}$ (and of course $q_{1}>q_{2}$ ), we will also, always have a different magnification in each direction of the detector's plane (where $M_{1}>M_{2}$, for $M_{n}=q_{n} / p_{n}$ ), what explains the difference of radii of curvature on both mirrors too.

\section{III.1.1.b KB microscope acceptance}

$\mathrm{KB}$ microscopes are generally designed to operate in the radiation range between soft and hard $\mathrm{x}$-rays (up to few tens of $\mathrm{keV}$ ) while maintaining a reasonable value of reflectivity (defined as the ratio between the incident and reflected photon number), and a reasonable acceptance solid angle for the instrument. According to Fig.III.1 we can define the acceptance as

$$
A_{c c_{n}}(E)=\int_{0}^{L} R_{E}\left(\alpha_{i}\right) \sin \left(\alpha_{i}\right) \frac{d x}{p_{n}}
$$

This relation takes into account the decreasing of the effective reflecting surface which is nothing but the projection of the mirror surface in the direction perpendicular to the incoming rays. The expression takes into account as well the specular reflectivity function $R_{E}$, for x-ray photons of fixed energy $E$, as a function of the grazing incidence angle $\alpha$. Here, $x$ refers to the position of the source to the reflecting point on the mirror (which must be integrated between 0 and $L$, the length of a single strip). We note that the higher the energy $E$ of the incoming photons, the smaller the grazing angle of incidence $\alpha_{i}$ must be and thus the effective reflecting surface is decreased. The total effective acceptance solid angle $\Omega_{e f f}$ for the photon energy $E$ is given by

$$
\Omega_{e f f}=A_{c c_{1}}(E) \times A_{c c_{2}}(E)
$$

where $A_{c c 1}(E)$ and $A_{c c 2}(E)$ refer to the first and second mirror respectively. We can then write

$$
N_{r e f}=F_{i n c} \times \Omega_{e f f}
$$

where $F_{\text {inc }}$ is the incident x-ray flux (photons/srad) and $N_{\text {ref }}$ is the total number of photons collected by the $\mathrm{KB}$ microscope and sent to the detector. 


\section{III.1.1.c Focal spot of the KB microscope}

Considering the rectangular aperture of the $\mathrm{KB}$ microscope, one can predict its focal spot size using the Fraunhofer diffraction formula for a slit aperture in each transverse direction given by

$$
I(\theta)=I_{0} \operatorname{sinc}^{2}\left(\frac{d \pi \sin (\theta)}{\lambda}\right)
$$

Where $I_{0}$ is the amplitude of the signal on the plane of the detector, $d$ is the mirror aperture size (where $d=L \sin (\alpha)$ ), $\lambda$ is wavelength of the incoming beam, $\alpha$ is the angle of deviation off axis. From the small angle approximation relation $(\sin (\alpha) \approx x / f)$, where $f$ is the focal length and $x$ the lateral displacement from the central axis on the plane of the detector, we can write

$$
I(x)=I_{0} \sin c^{2}\left(\frac{d \pi x}{\lambda f}\right)
$$

The expected, diffraction limited focal spot size, is always smaller the one measured experimentally. This effect is basically due to the effective aperture size $d_{\text {eff. The latter can be defined as }}$ the mirror aperture size which actually contributes to the focal spot size. This definition allows us to calculate the mirror efficiency $\eta$, given by the ratio between the effective aperture size $d_{\text {eff }}$ of the experimental fitted signal and the real one $d$.

$$
\eta_{\mathrm{i}}=\frac{d_{\mathrm{eff}, \mathrm{i}}}{d}
$$

Here the index $i$ can be 1 or 2 and refers to the first and second mirror of the KB microscope. The total estimated efficiency can be written

$$
\eta_{\mathrm{KB}}=\eta_{1} \eta_{2}=\frac{d_{\mathrm{eff}, 1} d_{\mathrm{eff}, 2}}{d^{2}}
$$

\section{III.1.2 The "Adjustable" KB microscope}

\section{III.1.2.a Motivation, inputs and conception}

Now that we have described the general physics of conventional KB microscopes, let us define in detail the characteristics of the X-ray instrument we are about to design. It is important to stress that most of KB microscopes are used in synchrotron facilities [Iida and Hirano, 1996, Krenkel et al., 2015, Hong et al., 2016]. In such installations, KB microscopes are generally conceived to transport specific $\mathrm{x}$-rays of narrow and determined bandwidth. In these circumstances, $\mathrm{KB}$ microscopes are generally placed at permanent positions where the focal distance is fixed (basically due to the fixed radii of curvature of the mirrors). In addition, the angle of incidence of $\mathrm{x}$-rays on the $\mathrm{KB}$ mirrors is practically pre-determined for each prototype. This degree of freedom reduces strongly the number of applications each instrument can have.

Since this thesis is more focused on the transport and applications of laser-driven x-ray sources, we need to design an x-ray optic suitable for such ultra-short and broadband radiation. Also, knowing that the device will be implemented at a laser facility, where experiment layouts are 
changing routinely, important criteria such size and positioning flexibility need to be considered for an optimum design.

These constraints, in addition to the ones cited in Sec.III.1.1, eliminated automatically the possibility of using Fresnel zone plates or Bragg crystals, leaving the KB microscope as the only candidate to transport efficiently broadband laser-driven x-rays. Considering both this fact and also the limitations that can have a KB microscope of fixed focal distance, makes of the adjustable KB microscope "the optimum solution".

The size of the $\mathrm{x}$-ray instrument is another important parameter to take also into account. This includes, the size of both, mirrors and $\mathrm{KB}$ microscope assembly. Considering, as an example, the existing KB microscopes of synchrotron facilities, we would find that most of them are of a relatively big size (from few tens of centimetres up to meter scale single mirrors). Taking into account that the usual size of vacuum chambers used for laser-plasma experiments are of the order of few tens of centimetres, makes such big optics spatially not adapted. Facing this challenge, implementing small size mirrors is the best solution for a more compact $\mathrm{KB}$ microscope design.

Adjustability is one of the most important requirements that our $\mathrm{KB}$ microscope design must to have. This can be satisfied by implementing a system able to modify and control the focal distance independently for each mirror, which implies the use of thin, flexible and flat materials. However, conventional KB microscopes use millimeter thick materials (glass, metal, mosaic) as mirror substrate, limiting remarkably the bending possibilities. Being highly flat and flexible (elastic), mono-crystal silicon plates match perfectly the requirements for a possible KB microscope mirror substrate. Indeed, silicon has been widely used, since decades, for the industry of electronics [Bryzek, 1991, Hochberg and Baehr-Jones, 2010]. The development and the manufacturing process of silicon plates have improved both, the purity and optical quality of these mirrors from one side, while the production cost has decreased from the other side. All these inputs have been sufficient enough to choose silicon for this purpose.

Silicon strips of $2 \mathrm{~cm} \times 10 \mathrm{~cm}$ with thickness $500 \mu \mathrm{m}$ were used as mirrors for the adjustable KB microscope. Such thin plates bend easily (see the simulations in next section, Fig.III.4) and offer a large flexible focusing range. These mirror dimensions permits to have a compact KB microscope assembly, $21 \mathrm{~cm} \times 31 \mathrm{~cm} \times 27 \mathrm{~cm}$, adapted to fit reasonably in laser-plasma experimental layouts.

It is noteworthy to mention that silicon is a commonly used mirror for soft $\mathrm{x}$-rays applications (below $1 \mathrm{keV}$ photon energy), but it's also important to know that silicon has reasonable reflectivity only at ultra small grazing incidence angles, which reduced strongly the acceptance (defined previously in Sec.III.1.1.b) of the x-ray optic. For this reason, the silicon strips were coated with a thin platinum layer $(\sim 50 \mathrm{~nm})$. The high electron density of platinum makes it suitable for use at larger angles of incidence and higher photon energies (up to tens of $\mathrm{keV}$ ), if compared with pure silicon. Results of calculated reflectivity maps for layers of 100nm thickness for both silicon and platinum as a function of incident $\mathrm{x}$-ray photon energy and grazing incidence angle are shown in Fig.III.2.

Knowing that the $\mathrm{KB}$ microscope is going to perform for high power laser-plasma related experiments, where the laser-driven x-ray sources are expected to be highly energetic (greater than $1 \mathrm{keV}$ ) and broadband (few $\mathrm{keV}$ ), adding the platinum coating layer is perfectly satisfying this requirement according to Fig.III.2. 


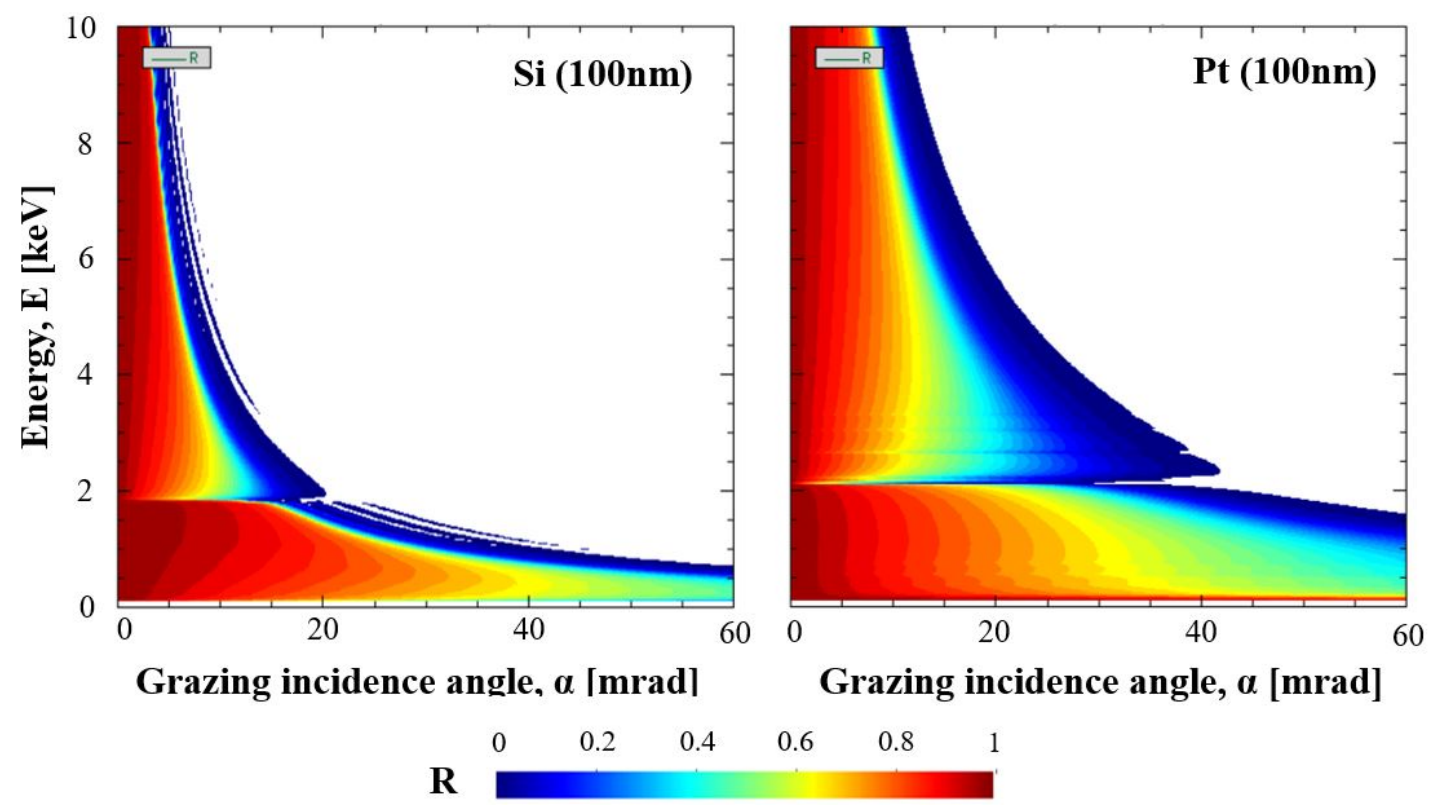

FIGURE III.2: Calculated reflectivity maps for a layer of 100nm thickness for silicon (left) and platinum (right), as a function of the grazing incident angle $\alpha_{i}$ and the $\mathrm{x}$-ray photon energy.

\section{III.1.2.b Mechanical design and simulations}

We present in this section an x-ray diagnostic equipped with two bendable mirrors, separately installed on independent benders. A bending technique, based on the "cantilever" mechanism principle (described in detail in [Howells, 2000]), was chosen for the design and optimized through numerical simulations of the design. This technique consists of applying an axial force on what is called the "leaf spring" of the bender. These leaf springs transfer the mechanical momentum to force at the mirror level, which consequently bend it. This bending technique permits to modify (transversally) the radii of curvature of the mirror without moving it longitudinally (in contrary to the "s-spring" bending mechanism [Howells, 2000]). Fig.III.3 is showing the principle components for the cantilever bending mechanism.

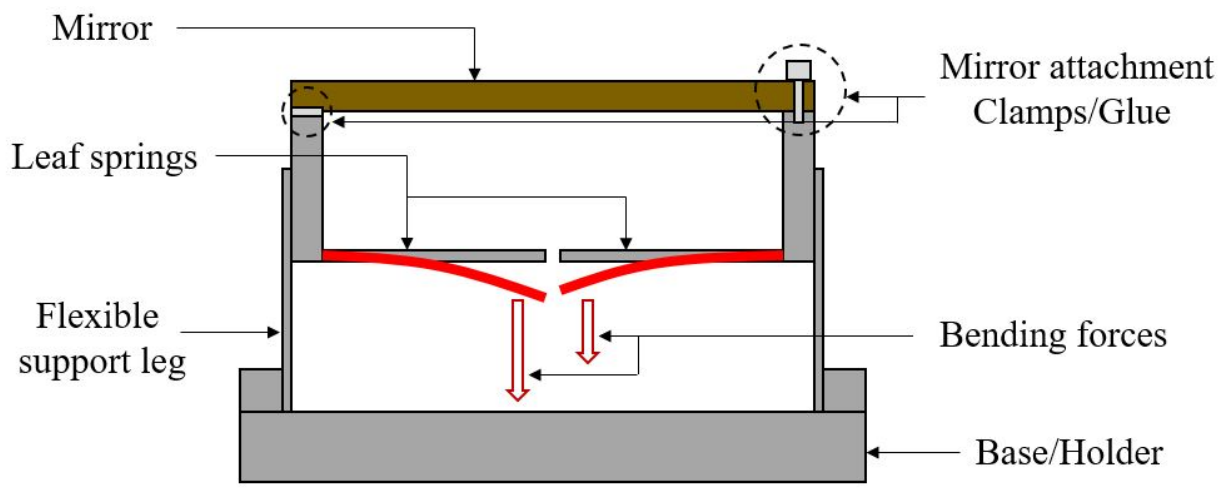

FIGURE III.3: The cantilever mechanism used to bend the mirrors of the adjustable KB microscope. Inspired from [Howells, 2000]. 
In order to increase the acceptance of the $\mathrm{KB}$ microscope, the latter should be placed as close as possible to the $\mathrm{x}$-ray sources. This implies a shorter mirror focus, which consequently means that the mirrors should have a shorter radii of curvature. Theoretically, for a mirror of $10 \mathrm{~cm}$ length, a displacement from the initial position, at the center of the mirror, of the order 120 microns, corresponds to a radii of curvature of the order of $10.5 \mathrm{~m}$. Such small radii of curvature allows for more fitting possibilities in experimental layouts (the KB microscope can be then placed at reasonable distances from the source, of the order of several tens of centimetres). According the stress (displacement) simulations, presented in Fig.III.4, 20N is enough to bend the silicon mirror to an almost perfect circle shape while displacing transversally its center of more than $100 \mu \mathrm{m}$ from the initial position.

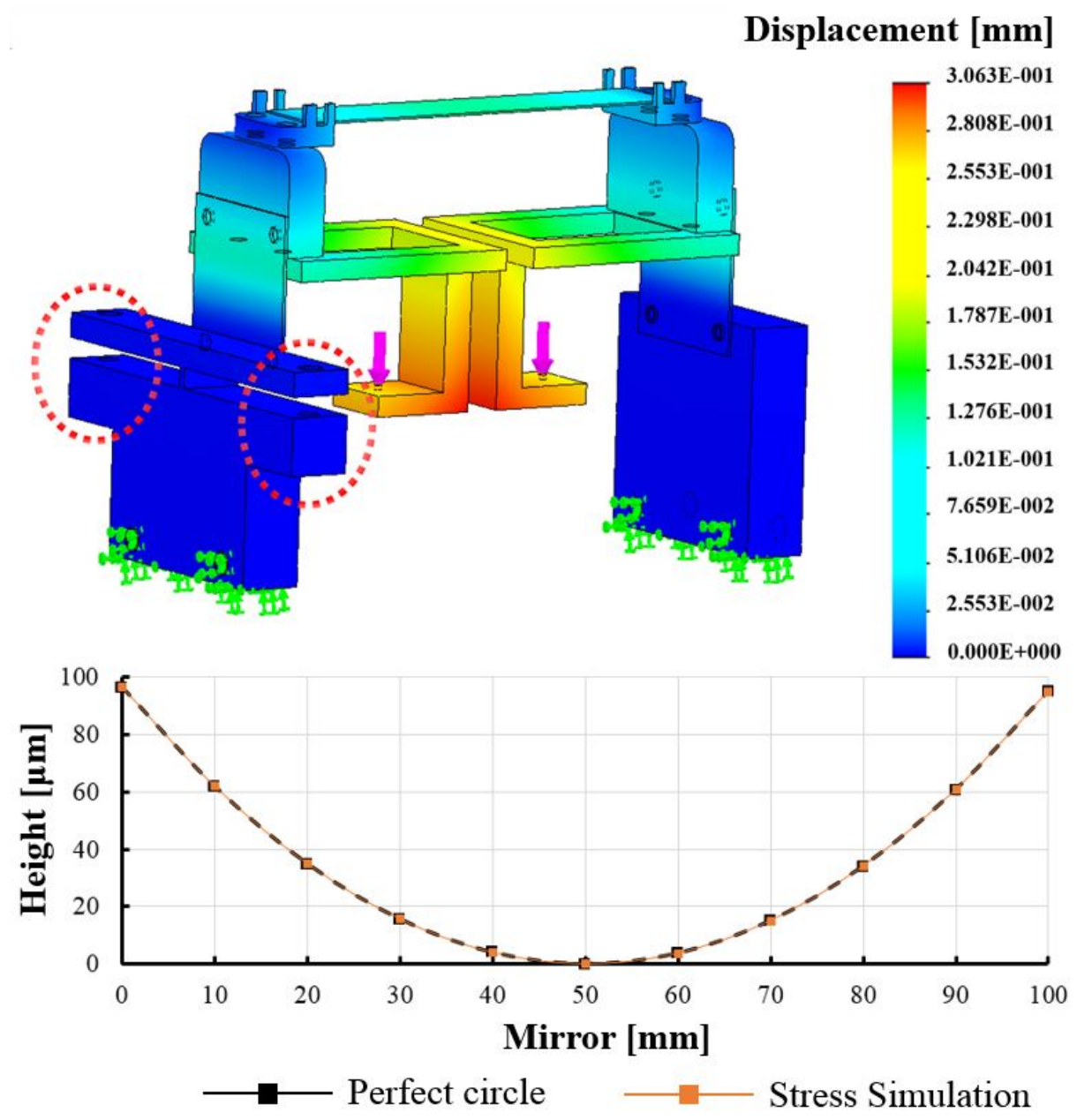

FIGURE III.4: (Top) Simulation for the displacement of the elements of a signle bender after applying a $20 \mathrm{~N}$ force (pink vectors) on the pushing arms. The twisting arms are also seen inside the dotted red circles. (Bottom) A comparison between the mirror profile after bending and a fitted perfect circle.

According to the numerical calculations (in Fig.III.4), we have determined less than $0.6 \mu \mathrm{m}$ deviation from a perfect cylindrical surface by measuring the surface profile calculated after applying a $20 \mathrm{~N}$ force to the bending actuators. The detailed difference between a simulated profile and a perfect circle profile is shown in the plots of Fig.III.5. The results indicate that the surface profile 


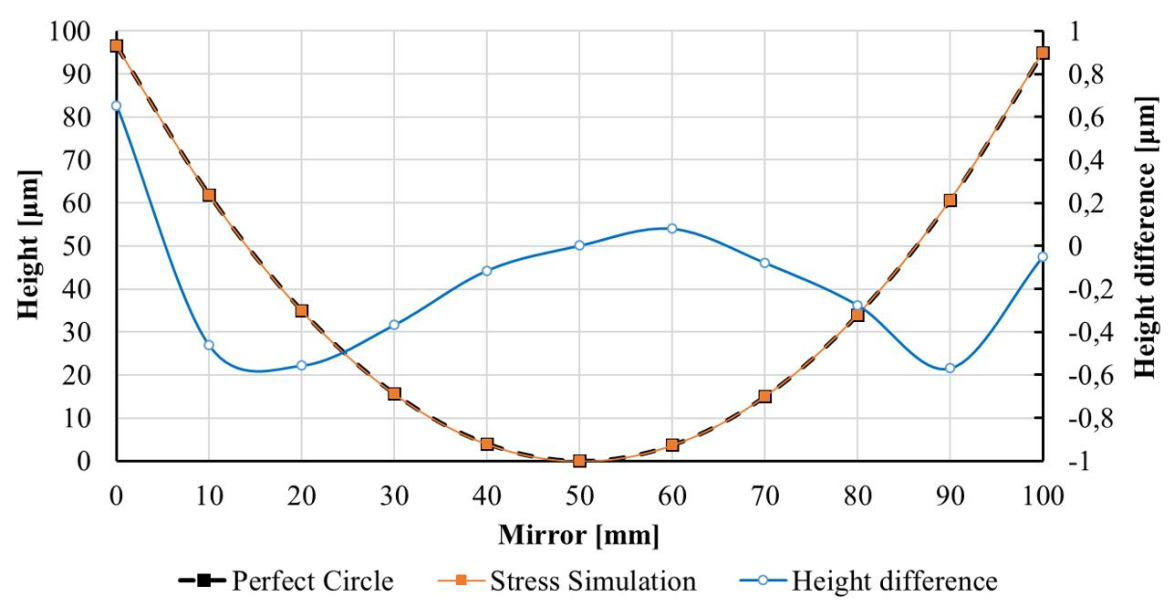

FIGURE III.5: Comparison between the mirror profile after bending using simulations and a perfect circle.

agrees within plus or minus $0.6 \mu \mathrm{m}$ with a perfect circle. We believe we are approaching the accuracy limit of the modeling code and some of the error may in fact be due to the numerical errors in the modeling calculation.

The design was made so that each bender is equipped with two, remotely controlled, piezoelectric actuator. Remote controlling is very practical for move the motors in vacuum. According to the data sheet of the Newfocus 8301 model (piezo-electric actuators), these motors can generate enough axial forces (up to $20 \mathrm{~N}$ ) to bend the silicon plate mirrors, matching perfectly our requirements. These actuators have also a step size of $30 \mathrm{~nm}$, offering a high degree of bending accuracy to the system. It is noteworthy to mention that each one of the benders was equipped with a manual twist adjustment system used to remove residual twist in the mirror (see red circles in Fig.III.4). Laboratory test have highlighted the importance of these twisting arms in both beam guiding and beam shaping.

Fig.III.6 shows a previously designed 3D scheme of a single bender. In this figure, we can clearly see the piezo-electric actuators in green colour. We can also see that the mirror is attached by clamps to the bender. It is noteworthy to comment that the attachment of the KB microscope mirrors to the bending mechanism represented a critical step in the assembly and many laboratory tests were carried out to determine the best way to do it. Initially, mechanical clamping was implemented to facilitate the replacement of the mirrors. However, this system added significant surface tensions leading to strong optical aberrations. We decided finally to use epoxy resin because it allows rigid attachment while minimizing deformation of the mirrors.

We have also added additional lateral high precision screws to the benders holders in order to control both, the height and the grazing incidence angle on the mirror. We decided to not to automatize these ones because such adjustments can be done easily in air. Adding more motors for this purpose would increase the cost of the device without no important justification.

We designed the present $\mathrm{KB}$ microscope in such way the benders are oriented at 45 degree angles, relative to horizontal. FigIII.7 shows the adjustable Kirpatrick-Baez microscope, once mounted, in the target area of the Spanish Center for Pulsed Lasers (CLPU). This orientation aims for keeping the total angular deflection of the x-ray beam in the horizontal plane, permitting the 


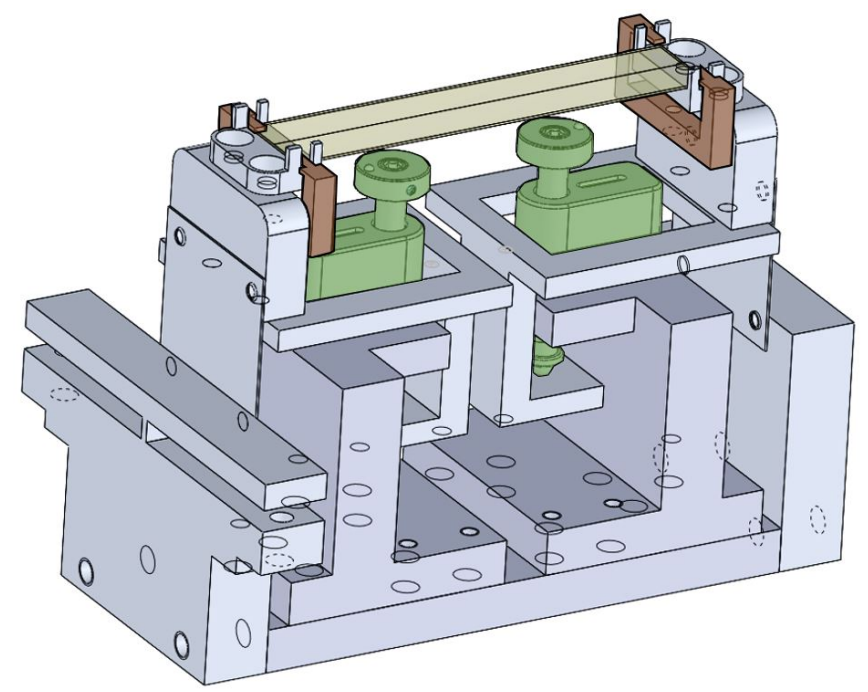

FIGURE III.6: The initial 3D design of a single mirror bender in which the binding (attachment) system mirror-to-bender was clamping (brown colour). The mirror can be seen in yellow meanwhile the actuators are highlighted in green.

detector to be mounted in the horizontal plane of the target system too. This property avoids installation/positioning problems, as is generally the case for conventional $\mathrm{KB}$ microscopes with mirrors oriented at $0^{\circ}$ degree and $90^{\circ}$ with respect to the horizontal plane.

\section{III.1.2.c Acceptance of the adjustable KB microscope}

One should be careful before implementing the KB mircoscope into an experiment. In fact, as we previously described in Sec.III.1.1.b, the goal would be to maximize the transport efficiency of the x-ray instrument. One should then consider the size of the mirrors, the coatings and the distance from the X-ray source. Fig.III. 8 shows plots of calculated reflectivity functions for various grazing angles of incidence and for two photon energies corresponding to the $\mathrm{K} \alpha$ emission of aluminium and copper for a single platinum coated mirror. The figure also shows the acceptance $A_{c c}$ of one mirror placed either at $0.5 \mathrm{~m}$ or at $1 \mathrm{~m}$ from a point-like x-ray source.

The real dimensions of the mirror $(2 \mathrm{~cm} \times 10 \mathrm{~cm})$ were considered to plot the curves of Fig.III.8. To simplify the calculations, the grazing incidence angle $\alpha$ was considered to be unvarying over the mirror surface $\left(d \alpha_{i} / d x=0\right)$. Thus, the grazing incidence angle $\alpha_{i}$ of Eq.III.2 corresponds then to the grazing angle at the center of the mirror. We note that the plots of Fig.III.8 were obtained for a perfect mirror, so, the real $\Omega_{e f f}$ (see Sec.III.1.1.b) must be corrected to take into account $\eta_{K B}$, the efficiency of the KB microscope mirrors, defined in Sec.III.1.1.c. For more precise calculations, values of roughness should be also taken into account.

According to Fig.III.8, we can see that the closer is the KB microscope to the X-ray source, the higher is the acceptance. This is straightforward since the numerical aperture/the solid angle of the device will be bigger closer to the source than if it is further. Knowing that the reflectivity depends on the grazing incidence angle, approaching the KB microscope to the $\mathrm{x}$-ray source would lead to a higher variance of the reflectivity along the mirror position. Thus, the estimation for a fixed angle of incidence over all the mirror is not valid any more, which would make 


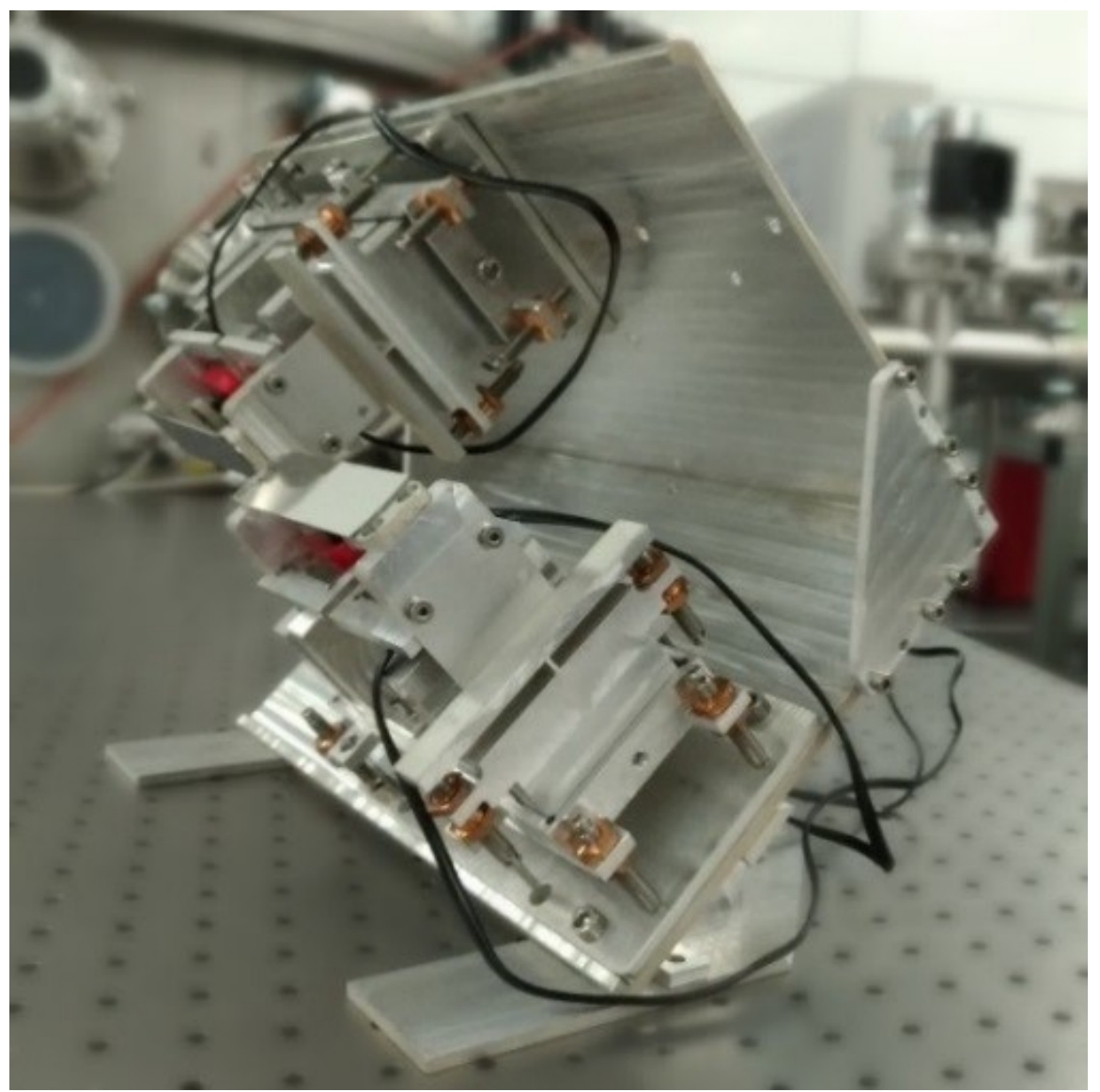

FIGURE III.7: Final assembly of the adjustable Kirkpatrick-Baez microscope.

the calculation of acceptance more complex. The second thing we can also notice is that the platinum coating is more efficient to reflect $\mathrm{Al}-\mathrm{K} \alpha$ than $\mathrm{Cu}-\mathrm{K} \alpha$. However, this can be noticed by simply looking at the calculated reflectivity curve of platinum. So, the final and most important parameter we can deduce from calculating the acceptance (in Fig.III.8) is the angle maximizing it. Indeed, the maximum of the acceptance curves represents the optimum compromise between the instrument's aperture and high reflectivity values. This calculation is essential before adjusting the KB microscope during the experiment. The angle maximizing the acceptance, and hence the number of photon reflected, using platinum coated mirrors is $32 \mathrm{mrad}$ and $0.9 \mathrm{mrad}$ respectively for $\mathrm{Al}-\mathrm{K} \alpha$ and $\mathrm{Cu}-\mathrm{K} \alpha$.

\section{III.1.2.d KB microscope alignment procedure}

The adjustments of the KB microscope varies from one experiment to another. For example, in an experiment where the main radiation to be transported is $\mathrm{Al}-\mathrm{K} \alpha$, the $\mathrm{KB}$ microscope needs to be adjusted to a specific grazing incidence angle $\alpha_{A l-K \alpha}$, maximizing the acceptance of the instrument (defined previously), different from $\alpha_{C u-K \alpha}\left(\neq \alpha_{A l-K \alpha}\right)$, the optimum grazing angle to reflect the $\mathrm{K} \alpha$ emission line of copper. 

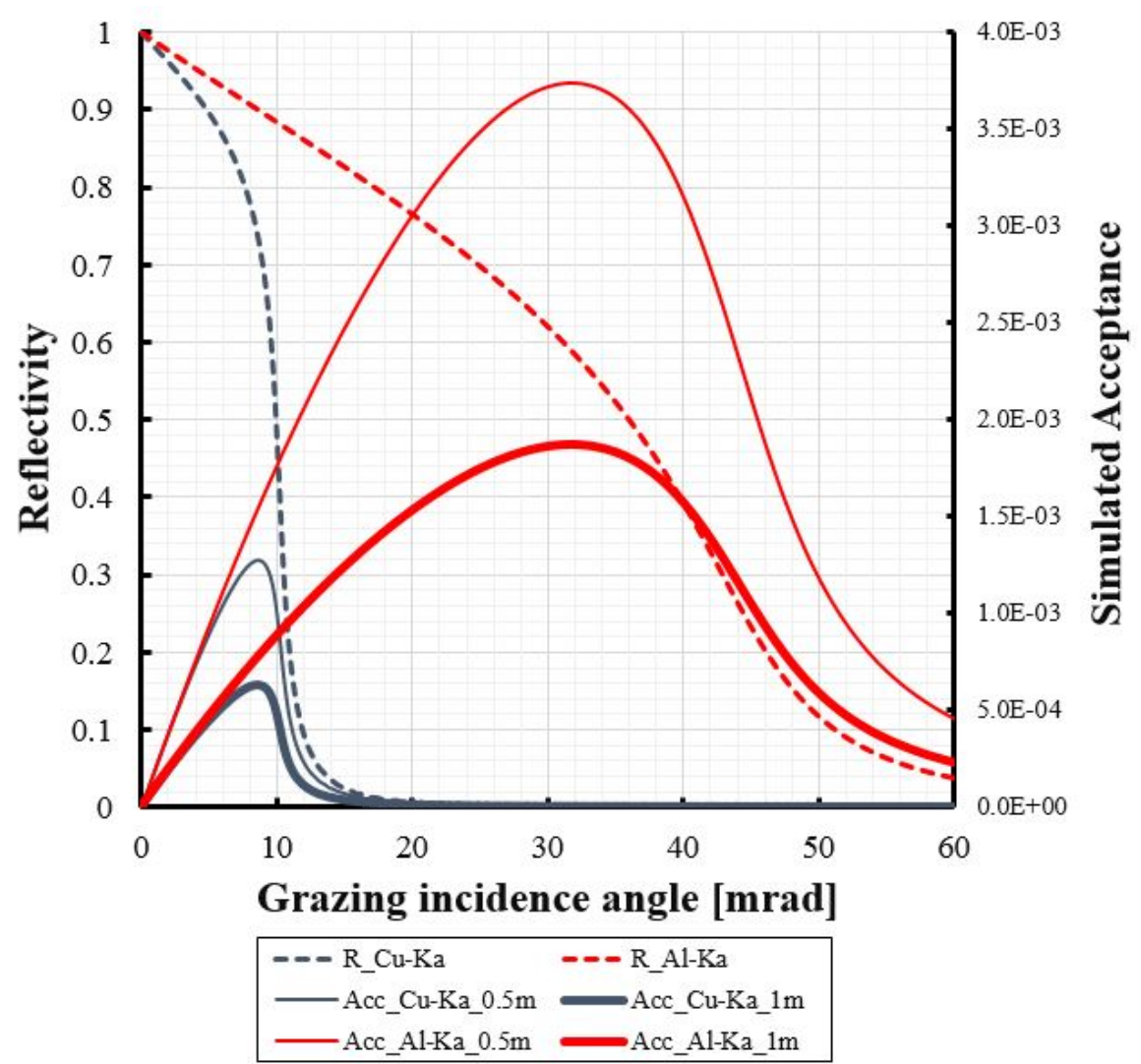

FIGURE III.8: Reflectivity functions of $\mathrm{Al}-\mathrm{K} \alpha(1.55 \mathrm{keV})$ and $\mathrm{Cu}-\mathrm{K} \alpha$ ( $8.05 \mathrm{keV}$ ) on a Pt coated mirror (dashed line). Acceptance $A_{c c}$ of a single $10 \mathrm{~cm}$ long Pt coated mirror at $1 \mathrm{~m}$ and $0.5 \mathrm{~m}$ for $\mathrm{Al}-\mathrm{K} \alpha$ and $\mathrm{Cu}-\mathrm{K} \alpha$ (solid line).

Defining the parameters shown in Fig.III.9, is of great importance for proper alignment of the KB microscope (at the right grazing incidence angle $\alpha_{i}$ ). We note that the theoretical value of $\alpha_{i}$ must be calculated/simulated and fixed before the starting of the adjustments.

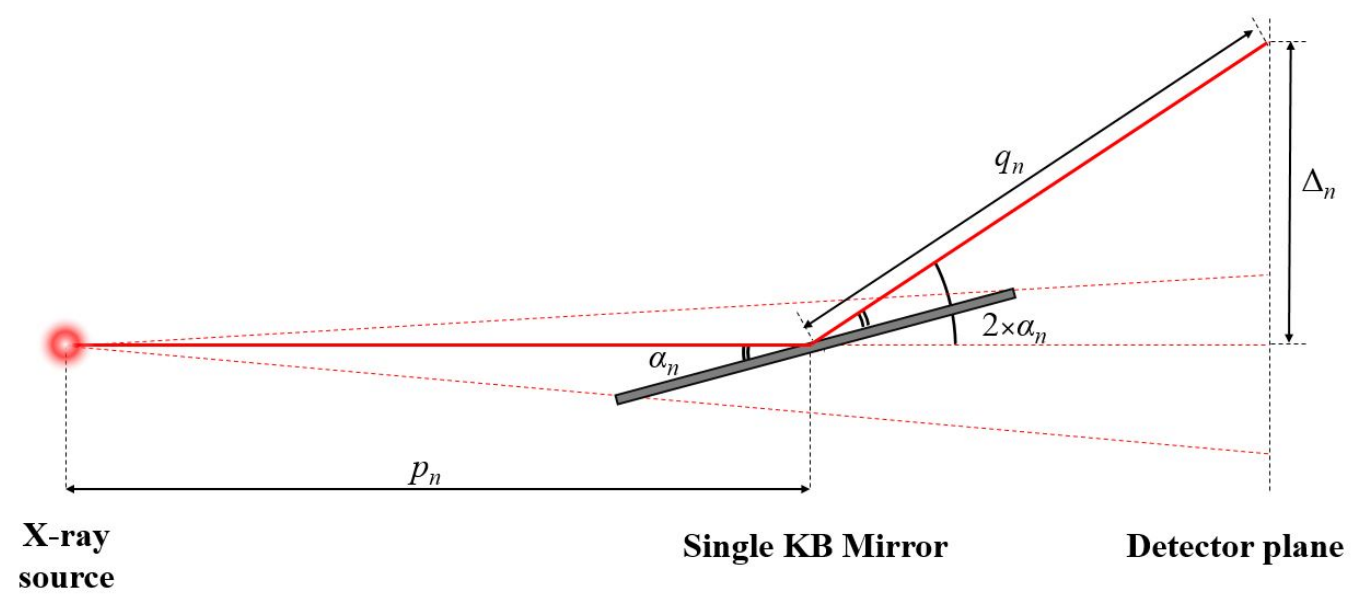

FIGURE III.9: Parameter definition for the KB microscope alignment. 
We usually use a laser (in the visible range) to carry out the macroscopic alignments concerning the KB microscope positioning. The microscopic adjustments are carried out later via the piezo-electric actuators movements and concerns the focusing of the beam (an example of beam focusing is presented in the KB microscope application section). We also note that for simplification reasons, we use $\alpha_{i}=\alpha$. According to Fig.III.9 and using the small angle approximation relation $\left(\sin \left(2 \alpha_{n}\right) \approx 2 \alpha_{n}\right)$ we can define

$$
\alpha_{n}=\frac{\Delta_{n}}{2 q_{n}}
$$

Only by measuring the separation between the chief ray and the position of the deviated ray, one can define experimentally $\alpha_{n}$. Varying the distance $\Delta_{n}$ can be carried out via the adjustments of the lateral micrometer screws (clearly seen in FigIII.7). Fig.III.10 shows how the rays used for alignment experimentally look like.

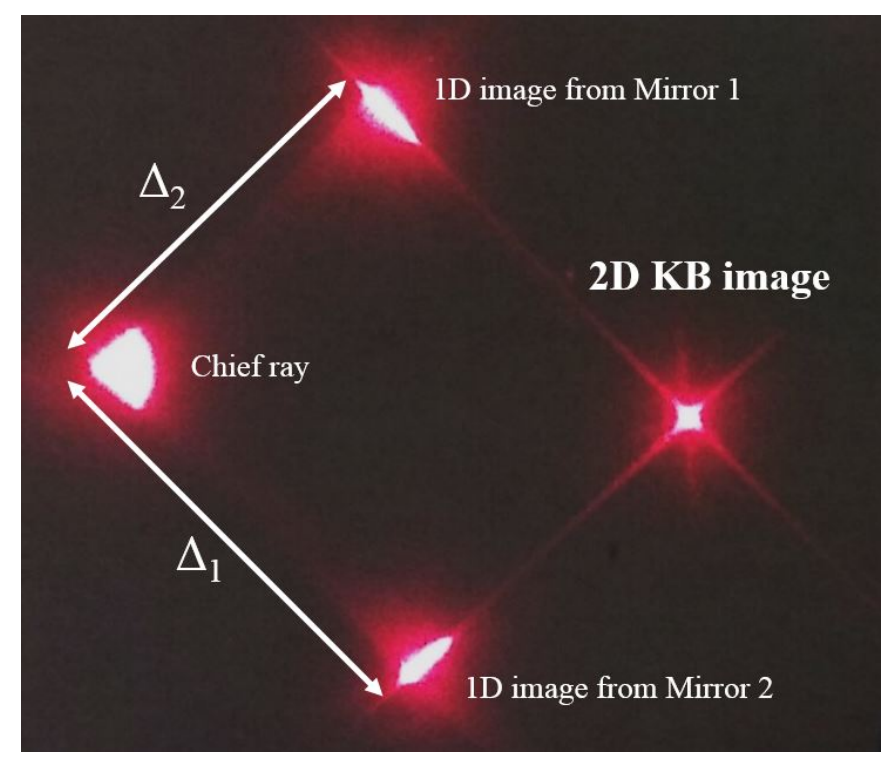

FIGURE III.10: The final 2D image of an adjusted KB microscope next to two 1D images obtained from mirror 1 and 2 separately. The reference chief ray is also shown here.

We can also remind that since $M_{1}>M_{2}$, we will always have $\Delta_{1}>\Delta_{2}$, which explains the rectangular shape formed by the four regions of interest in Fig.III.10.

\section{III.2 Optical characterisation of the KB microscope}

\section{III.2.1 Characterisation of the mirrors flatness}

The mirrors installed in our KB system are platinum coated silicon plates of $500 \mu \mathrm{m}$ thickness. The novelty about this technique is that nobody has ever used this type of mirrors before. However, using a new technology requires detailed characterisation of the performance. We have 
decided to use an optical technique to characterize the surface of the mirrors. The optical measurements at normal incidence were used to characterise the surface shape from measuring the angular deviation of the reflected rays. The experimental setup used to carry out this measurements is shown in Fig.III.11.

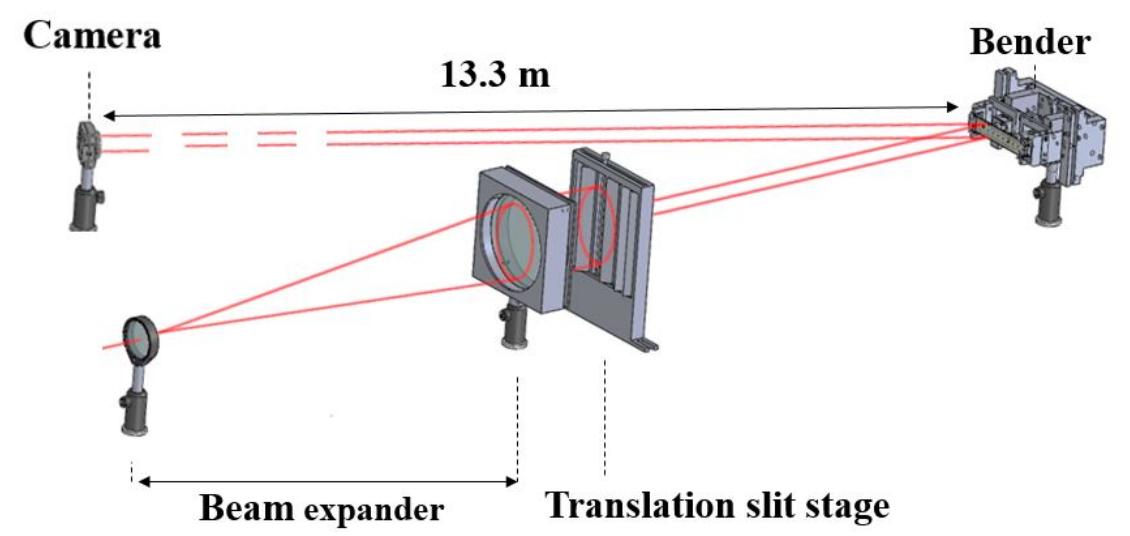

FIGURE III.11: Experimental setup for mirror surface characterisation.

The experimental setup consists of expanding a He-Ne $(632 \mathrm{~nm})$ laser beam to a $10 \mathrm{~cm}$ wide beam, equal to the length of the strips. The $10 \mathrm{~cm}$ diameter beam was truncated by a $1 \mathrm{~cm}$ slit placed on a translation stage. The latter used for scanning of the mirrors i.e cutting the beam into slices of $1 \mathrm{~cm}$ wide for an independent measurement of each region of the mirror. Each bender was considered separately to carry out the measurements. After reflection, the beam was collected by a camera placed at $13.3 \mathrm{~m}$. The silicon plate was bent to give a focus at approximately $13.3 \mathrm{~m}$. The followed steps used to carry out the measurements are shown in Fig.III.12.

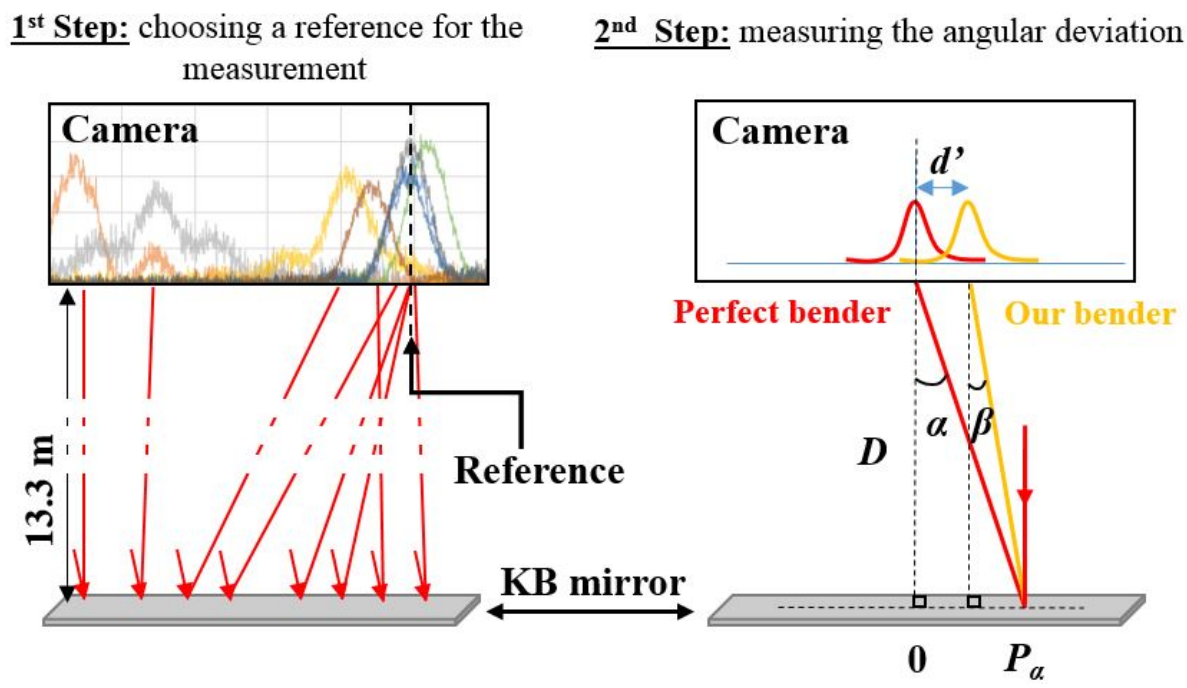

FIGURE III.12: Definition of parameters for the measurement of the angular deviation.

We can define the slope error as the angle difference between the tangent of a perfect cylindrical 
mirror and the tangent at the real mirror at each position. The slope error was calculated by measuring the angular deviation for each $1 \mathrm{~cm}$ segment of the mirror. The results of the measurements can be seen in Fig.III.13.
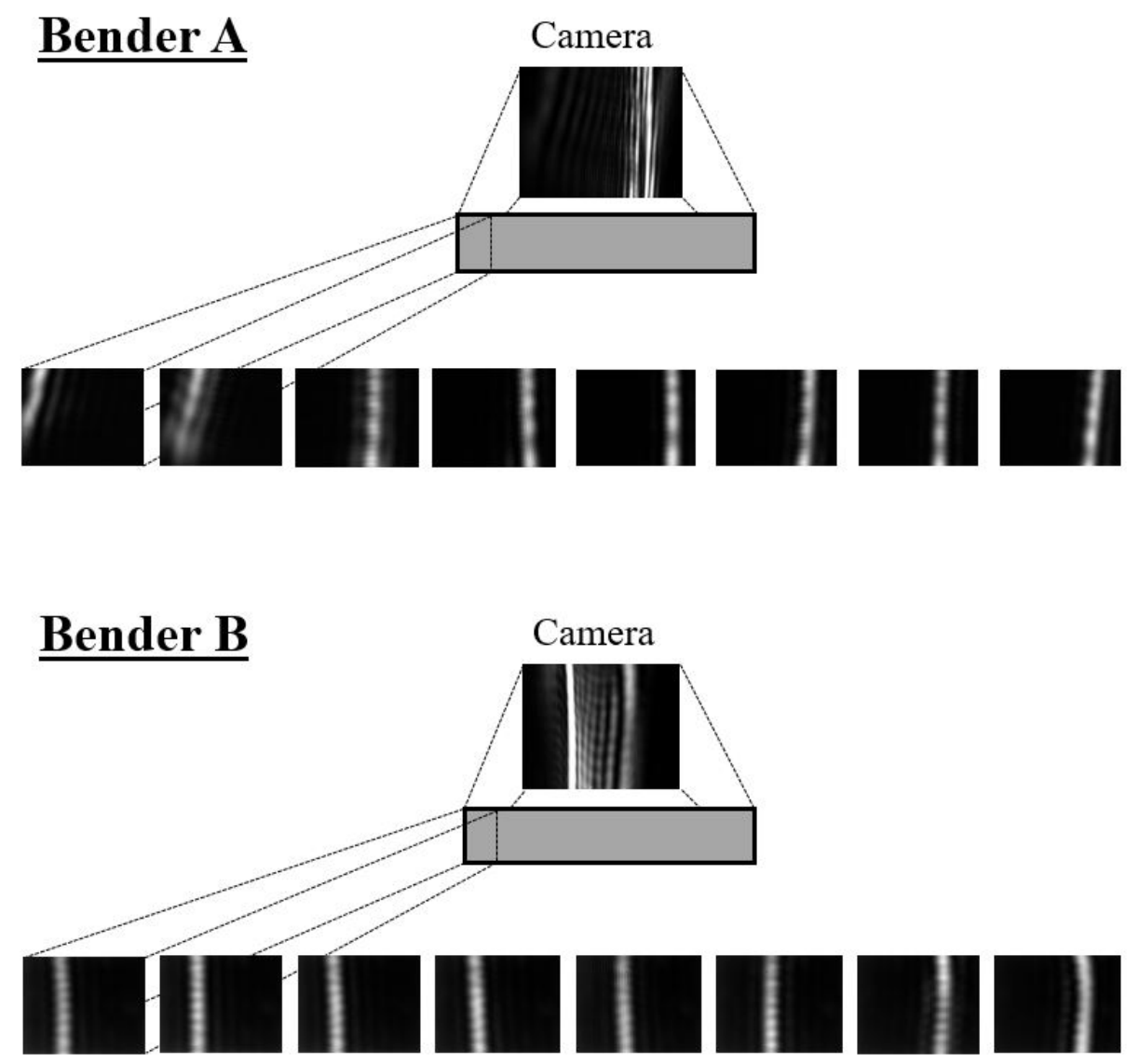

FIGURE III.13: (Bottom) Experimental results of the focus of isolated mirror segments on the camera for both mirrors. (Top) Sum of all rays on the camera (when the translation slit is fully open, $10 \mathrm{~cm}$ wide aperture).

From the data of Fig.III.13, it is clear that each mirror has it own "behaviour"/response in terms of deflection patterns corresponding to the specific shape of each mirror. This difference of shape is due to the proper flatness irregularities of the silicon plates. According to Fig.III.12 we can express the angular deviation as

$$
\Psi=\left(\frac{\alpha-\beta}{2}\right)
$$

Where

$$
\begin{gathered}
\alpha=\tan ^{-1}\left(\frac{P_{\alpha}}{D}\right) \\
\beta=\tan ^{-1}\left(\frac{P_{\alpha}-d^{\prime}}{D}\right)
\end{gathered}
$$

$\alpha$ and $\beta$ are the angles measured from the focus position of a theoretical perfect bender and our measured bender, respectively. $P_{\alpha}$ refers to the position of the incident beam on the mirror 
surface, $D$ is the distance from the camera to the mirror and $d^{\prime}$ the separation between the real and the theoretical position of the image on the camera plane. Integrating the angular deviation in function of position (on the mirror) gives a residual error in surface height. Fig.III.14 shows the final result of the analysis where it can be clearly seen that the slope error is less than $100 \mu \mathrm{rad}$ over almost all the mirrors surface.

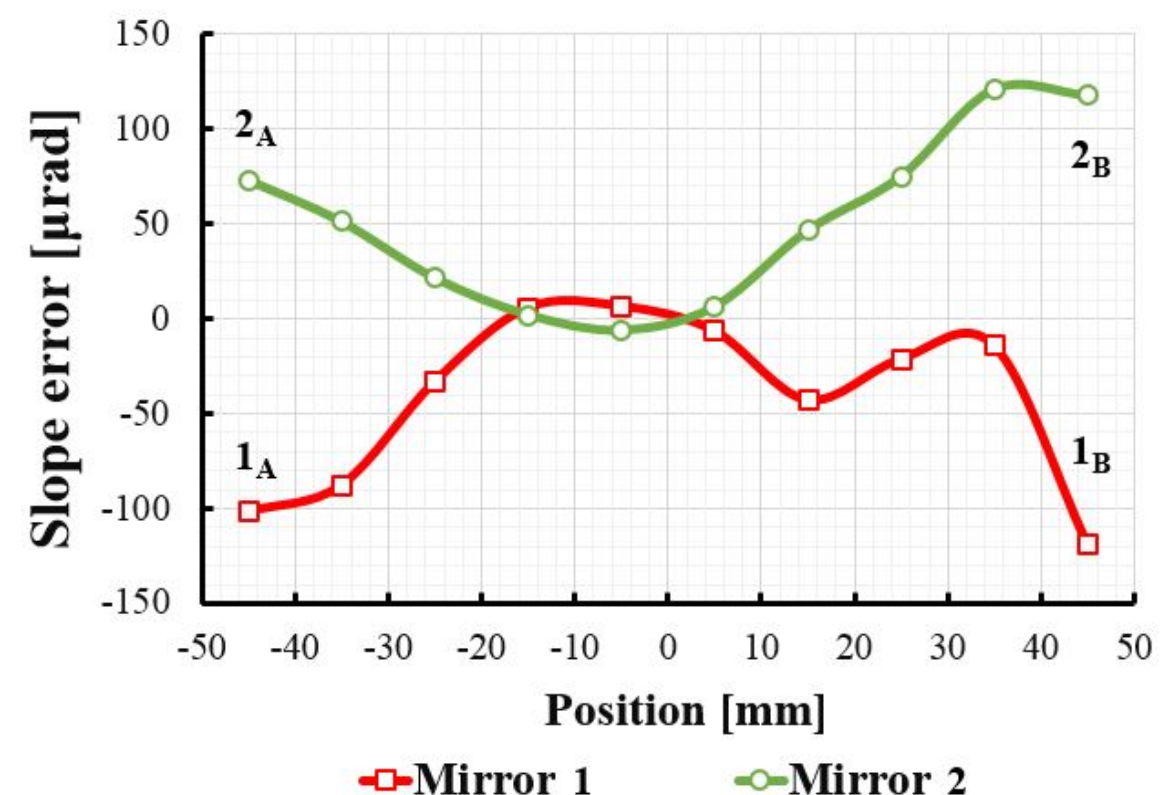

FIGURE III.14: Slope error measurement results of mirror 1 and mirror 2. The letters A and B refer to the edges of each mirror. Here $\mathrm{X}$-ray photons are assumed to hit mirror 1 first and then, after being reflected, mirror 2.

\section{III.2.2 Characterisation of the focal spot}

We have performed a study for image quality characterisation of the KB microscope by analysing the focal spot of the device. The KB assembly was installed in such a way the it focuses a collimated $632 \mathrm{~nm}$ He-Ne beam onto a camera. The goal was to optimise and then measure the $2 \mathrm{D}$ spot size obtained with the KB microscope. Adjusting the whole KB assembly to get the mirrors at a grazing incidence angle of $2^{\circ}$ (optimised for Al-K $\alpha$ emission) was the first alignment step. The focus was then adjusted to get the tightest achievable spot using the actuators of each bender. Fig.III.15 shows the experimental setup used for the optimisation of the focal spot.

We note that the distances indicated in Fig.III.15 were chosen in purpose to simulate a real operating case i.e. in vacuum, inside the existing CLPU's vacuum chambers, where no "relatively big" distances were considered since the setup in real experiments is supposed to be as compact as possible.

The focal spot obtained together with the plotted profiles for both bender 1 and 2 are shown in Fig.III.16.

The experimentally measured focal spot sizes (at FWHM) for the given distances (in Fig.III.15) using a He-Ne laser beam are $130.8 \mu \mathrm{m}$ and $108.5 \mu \mathrm{m}$ for the mirrors 1 and 2 respectively. For 


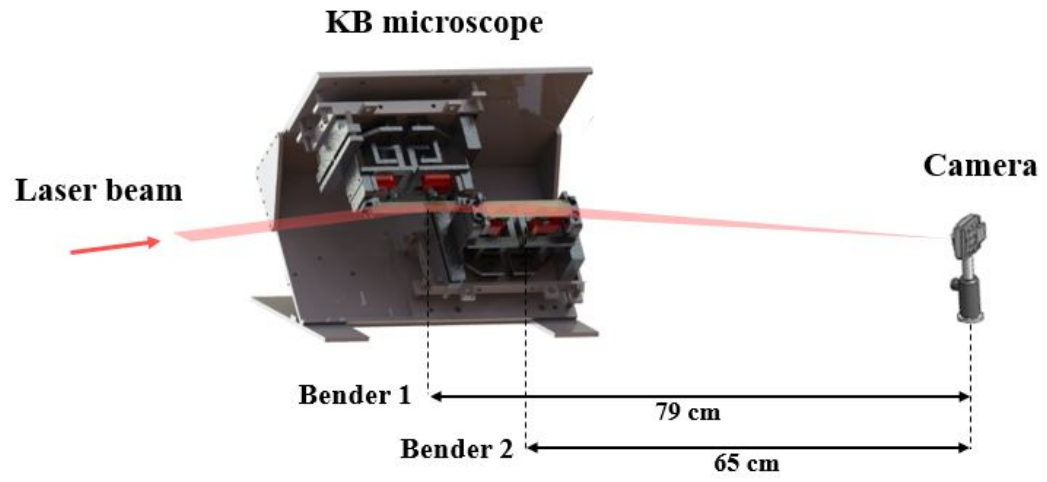

FIGURE III.15: Experimental setup for focal spot optimisation.
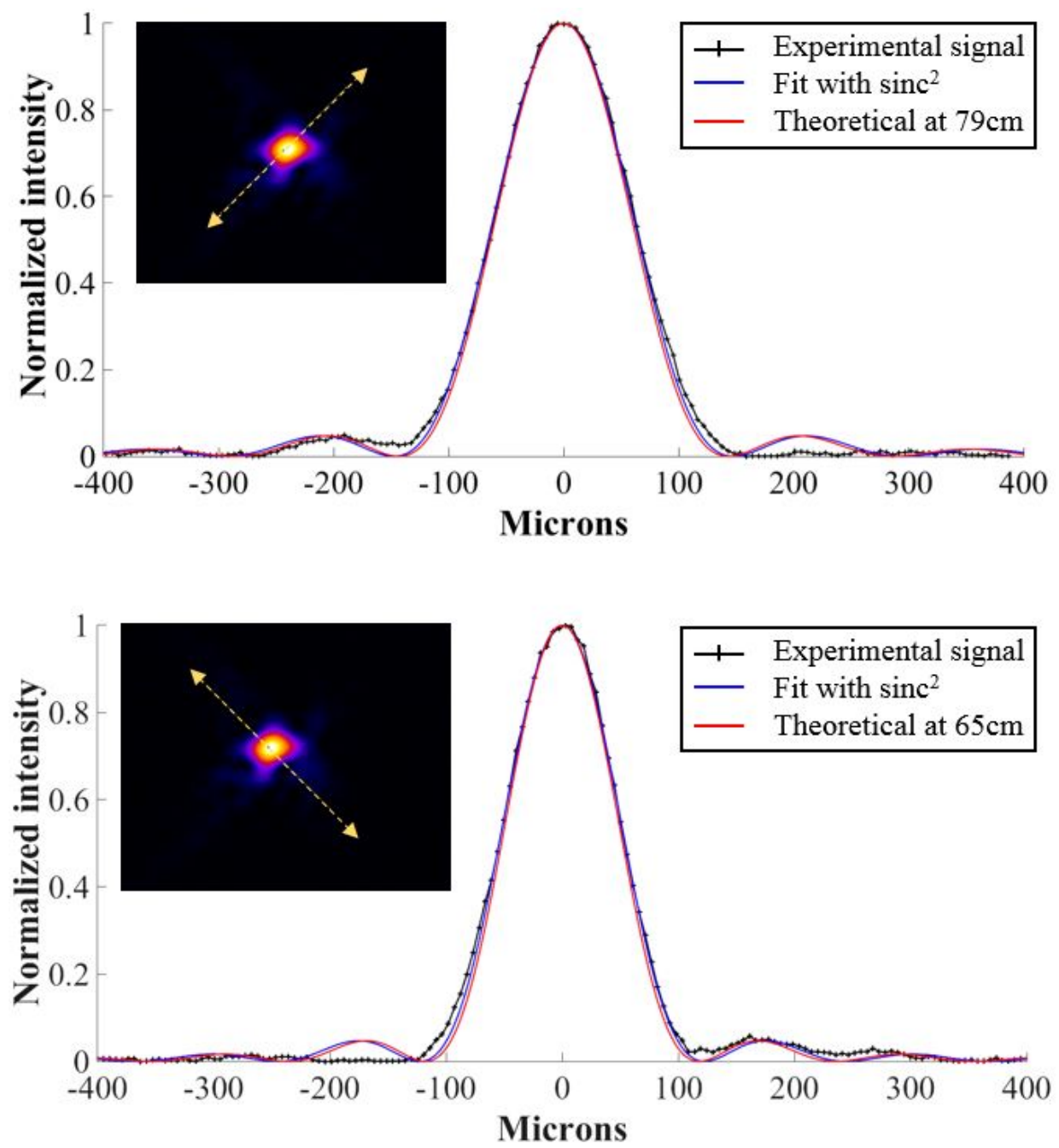

FIGURE III.16: Focal spot produced by the KB microscope and its profiles obtained in the direction determined by mirror 1 (top) and in the perpendicular direction determined by mirror 2 (bottom). 
comparison, the theoretical values were expected to be $126.9 \mu \mathrm{m}$ for mirror 1 and $104.4 \mu \mathrm{m}$ for mirror 2. Comparing the experimental measurements to the theoretical expectations gives a value of $\eta_{\mathrm{KB}}$ reaching $93 \%$.

Once the characterisation of the KB was completed, the device was then ready to be tested for laser driven $\mathrm{x}$-ray sources experiments. 



\section{Chapter IV}

\section{Experimental studies}

\section{Introduction}

We have explained, up to this point of the thesis, the main mechanisms ruling the generation of different laser-driven x-ray sources. Also, many diagnostics and optics operating in the x-ray range, have been introduced in the previous chapters for their important role in the experiments presented later in this chapter. Divided in two main parts and fully dedicated to experimental activities, this chapter summarises the main experiments to which I have participated. A first one shows in detail the $\mathrm{x}$-ray performances of the adjustable $\mathrm{KB}$ microscope, optically characterised in the previous chapter, and its role in laser-plasma interaction experiments. A comparison between the results obtained using the $\mathrm{KB}$ microscope and results using a spherical Bragg crystal in proton acceleration experiments using the VEGA-II laser (more details in Sec.IV.1.1) are also presented in this section of the thesis. The second half of this chapter is dedicated to the applications of laser-driven x-ray sources in the context of X-ray Phase Contrast Imaging (XPCI). Results obtained from two different experiments, independently carried out in CLPU and GSI (Sec.IV.1.2) facilities, implying the use of two different laser-driven x-ray sources are discussed. The first experiment consists of an application of XPCI using betatron-based x-ray source generated via LWFA in CLPU (shown in Sec.IV.4.2), while the second one is dedicated to the study of XPCI using bremsstrahlung radiation carried out at GSI Laser facility (in Sec.IV.4.3). I have been author of two peer reviewed articles [Zeraouli et al., 2019, Volpe et al., 2019] related to proton acceleration experiment 1 and to three more concerning the XPCI experiment 2 [Antonelli et al., 2019, Barbato et al., 2019a, Barbato et al., 2019b]. A summary of the these successful experiments together with others (still unpublished) are presented in this chapter.

\section{IV.1 High intensity laser facilities}

\section{IV.1.1 CLPU laser facility}

The Spanish Centre for Pulsed Lasers [CLPU, 2020, Méndez et al., 2019] (Centro de Láseres Pulsados, CLPU) is a national facility specialized on ultra-short (30fs) and ultra-intense laser pulses, located in the scientific park of Salamanca (Spain), is nowadays operating at Gigawatt Terawatt and Petawatt levels. The aim of the facility is to serve the scientific community and industry by providing access to high power lasers as well as advice through collaborative research. CLPU is an open facility to national and international users. CLPU laser beam line offers 
three front ends seeded by the same Ti-Sapphire oscillator (for femtosecond synchronisation reasons). Each of one of these front ends is amplified in a separate beam line (see Fig.IV.1) with the following output characteristics

\section{CLPU beam lines}

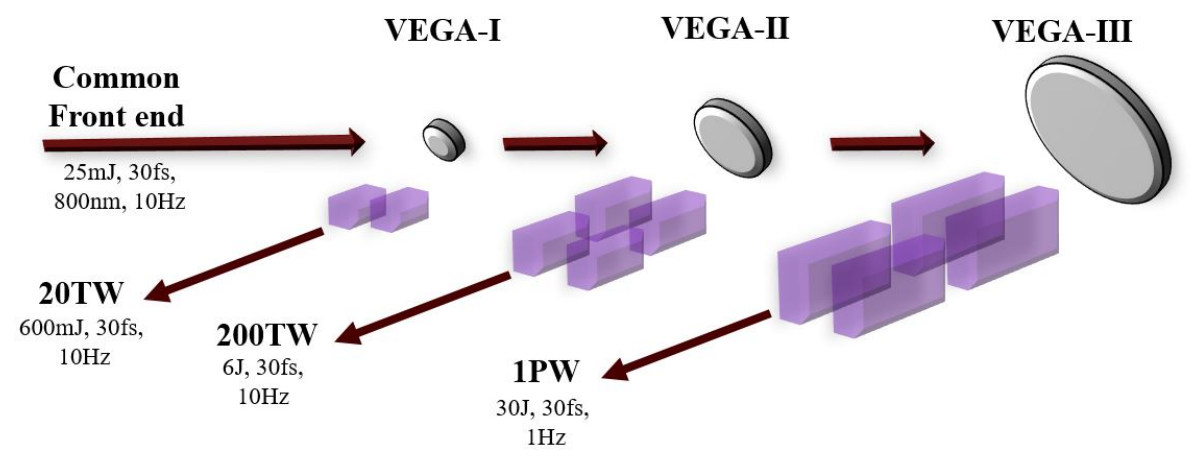

FIGURE IV.1: Main CLPU laser lines. Beam amplification and compression stations are shown in purple colour.

- VEGA-I: $800 \mathrm{~nm}$ central wavelength, $600 \mathrm{~mJ}$ energy per pulse, $30 \mathrm{fs}$ of pulse duration and can operate up to $10 \mathrm{~Hz}$. VEGA-I beam diameter at the exit of the compressor is $3.5 \mathrm{~cm}$.

- VEGA-II: 800nm central wavelength, 6J energy per pulse, 30fs of pulse duration and can operate up to $10 \mathrm{~Hz}$. The beam diameter of VEGA-II at the exit of the compressor is $10 \mathrm{~cm}$.

- VEGA-III: $800 \mathrm{~nm}$ central wavelength, 30J energy per pulse, 30fs of pulse duration and can operate up to $1 \mathrm{~Hz}$. VEGA-III beam diameter is more than twice bigger than VEGA-II with a size at the exit of the compressor of $25 \mathrm{~cm}$.

Proton acceleration experiments 1, 2, strong shocks generation experiment 1 and XPCI experiment 1 (presented in details in the next section) have been carried out using the same VEGA-II laser but with different focusing configurations (for application purposes) as shown in Fig.IV.2. Having the possibility to modify the laser focusing configuration (using parabolas with different $\mathrm{f} /$ numbers), is of great interest since two intensity regimes can be investigated. A few parameters corresponding to each laser focusing scheme for optimal theoretical conditions (for diffraction limited focal spots and laser parameter $M^{2}=1$ ) can be found in the table of Fig.IV.3

\section{IV.1.2 PHELIX laser of GSI facility}

PHELIX [Neumayer et al., 2005] stands for Petawatt High-Energy Laser for heavy Ion eXperiments and is a high intensity laser facility located in GSI Darmstadt, Germany, aimed at developing research in many fields as plasma and astrophysics, nuclear physics and atomic physics... PHELIX is a flash lamp pumped Nd:Glass laser with two frontends with energies up to kilojoule and power up to 500TW. PHELIX can deliver two ranges of laser pulses depending on the application. A long laser pulse frontend of 1-10ns duration with energies up to $1 \mathrm{~kJ}$ and a short one of 500fs-20ps duration with energies up to 200J. An interesting configuration in PHELIX consists of splitting the beam in two halves for pump and probe experiments as seen in Fig.IV.4. More 
Long Focal Parabola

Configuration

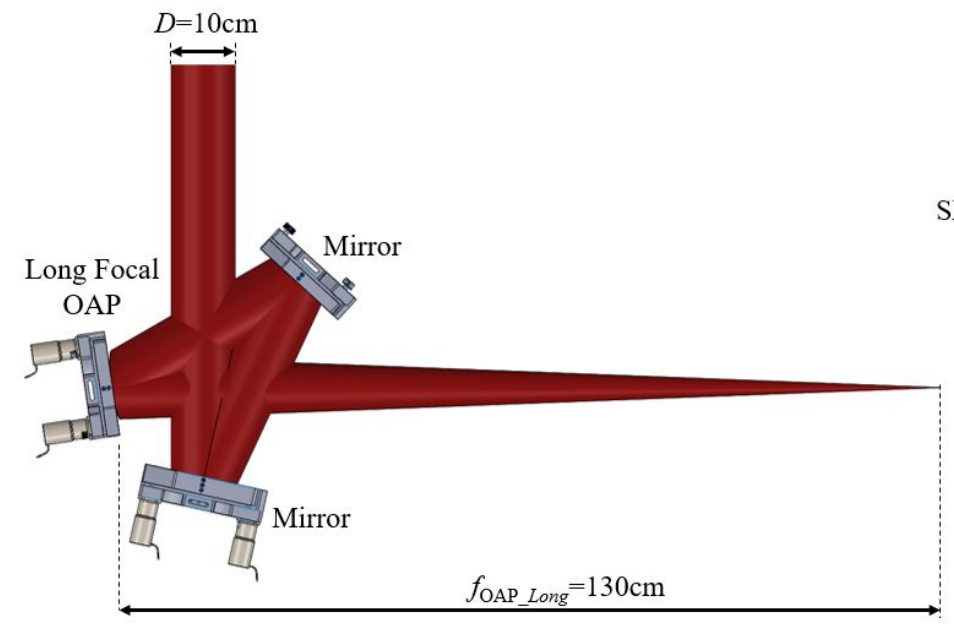

Short Focal Parabola

Configuration

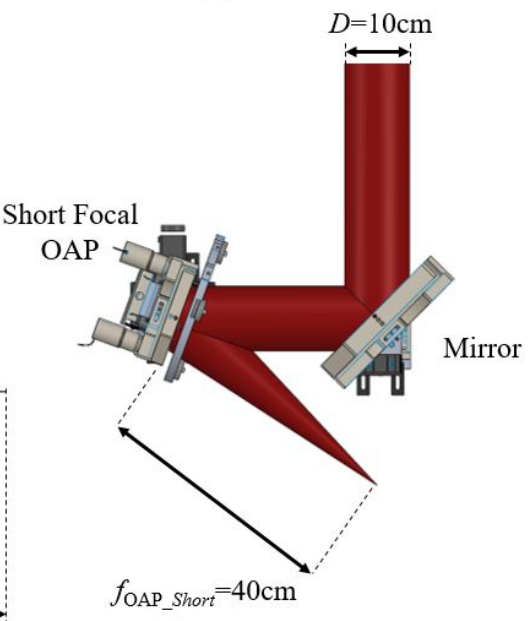

FIGURE IV.2: Possible laser focusing schemes for VEGA-II laser system.

\begin{tabular}{|c|c|c|c|c|c|c|c|c|}
\hline Laser & Configuration & $\begin{array}{c}\text { Laser } \\
\text { energy } \\
{[\mathrm{J}]}\end{array}$ & $\begin{array}{c}\text { Beam } \\
\text { Diameter } \\
{[\mathrm{cm}]}\end{array}$ & $\begin{array}{c}\text { Focal } \\
\text { distance } \\
{[\mathrm{cm}]}\end{array}$ & F/Number & $\begin{array}{c}\text { Focal Spot } \\
\text { Diameter } \\
{[\mu \mathrm{m}]}\end{array}$ & $\begin{array}{c}\text { Rayleigh } \\
\text { length } \\
{[\mu \mathrm{m}]}\end{array}$ & $\begin{array}{c}\text { Intensity on target } \\
{\left[{\left.\mathrm{W} . \mathrm{cm}^{-2}\right]}^{2}\right.}\end{array}$ \\
\hline \multirow{2}{*}{ VEGA-II } & Short & \multirow{2}{*}{6} & 10 & 40 & 4 & 4,08 & 16,31 & $1.53 \times 10^{\wedge 21}$ \\
\cline { 2 - 7 } & Long & & 130 & 13 & 13,25 & 172,23 & $1.45 \times 10^{\wedge 20}$ \\
\hline VEGA-III & Long & 30 & 25 & 250 & 10 & 10,19 & 101,91 & $1.22 \times 10^{\wedge 21}$ \\
\hline
\end{tabular}

FIGURE IV.3: Possible laser parameter for VEGA lasers in function of the laser focusing scheme.

details about the exact laser parameters used for an experiment (XPCI experiment 2) presented in thesis are shown later (see Sec.IV.4.3).

We note that even if both, PHELIX and VEGA, are PW class lasers, the applications related to each one can be completely different. The reason behind this is directly due to the pulse duration of each laser (low energy (30J) in an ultra short period of time (30fs) for the case of VEGA-III and a much higher energy (200J) in a longer period of time (500ps) for PHELIX). Another difference between the two systems is that VEGA can operate at high repetition rate while PHELIX operates only at single shot.

\section{IV.2 X-ray detection}

For almost every range of wavelengths in the electromagnetic spectrum detectors have been developed with different quantum efficiencies. Since this thesis is more focused on laser driven$\mathrm{x}$-ray sources and applications, we introduce two of the most used detectors by laser-plasma community in the soft $\mathrm{x}$-ray range (few $\mathrm{keV}$ up to $<100 \mathrm{keV}$ ): Imaging plates and $\mathrm{X}$-ray cameras. 


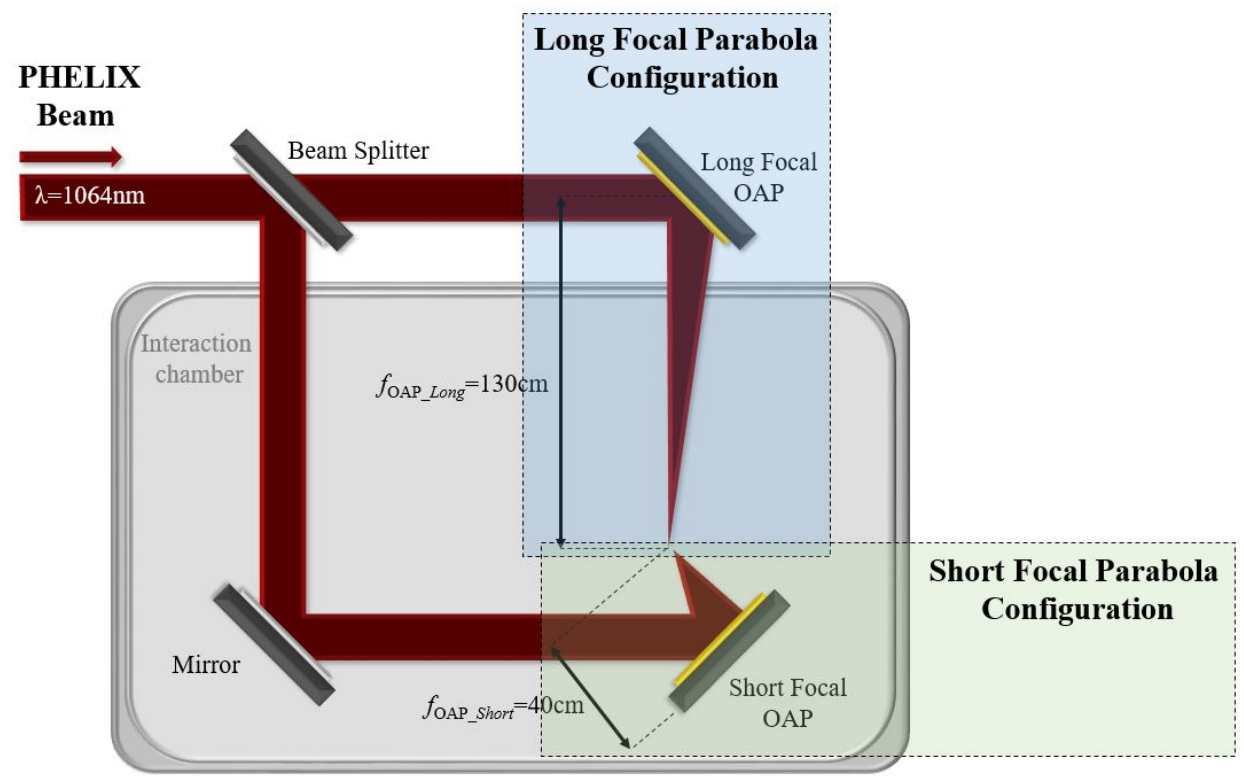

FIGURE IV.4: PHELIX high power interaction chamber.

Both of these two x-ray detectors have been used in this thesis. A case in which we compare the response of both detectors, placed together at the same time (see comparison between results for $\mathrm{XPCI}$ experiment 2), is also presented in this thesis.

\section{IV.2.1 Passive detectors: Imaging plates}

We can define imaging plates (IP) as flexible x-ray sensors consisting of a thin active layer of photo-stimulatable microscopic crystals (of few microns) embedded in plastic attached uniformly to a thicker plastic layer to create a solid assembly (generally polyester film) [Maddox et al., 2011] as shown in Fig.IV.5. A ferromagnetic layer (not shown in the figure) is usually placed before the Polyester layer allowing for a better IP handling during the scanning. The active layer consists of phosphor of barium fluorobromide with traces of bivalent europium, formulated as $\mathrm{BaFBr}: \mathrm{Eu}^{+2}$. Only an $\mathrm{x}$-ray irradiation can bring the europium $\mathrm{Eu}^{+2}$ to the ionised metastable state $\mathrm{Eu}^{+3}$ where the emitted electron is trapped into the $\mathrm{Br}$ ion empty lattices. A laser scanner, operating in the visible range (generally emitting in the red 633nm) delivers enough energy to excite the trapped electrons and bring them back to the $\mathrm{Eu}^{+2}$ ions, emitting a photon of higher energy, in the blue range. This light is called photostimulated luminescence or PSL. The amount of photons then collected is proportional to the number of x-ray photons hitting the IP detector. Experimentally, an IP can be used hundreds of times, but, one should be always careful on erasing all the signal from the previous $\mathrm{x}$-ray irradiation. This operation can be carried out easily by illuminating the IP with intense white light for a relatively long period of time $(\sim$ to 30min). Fig.IV.5 shows the steps for using IP sensors. 


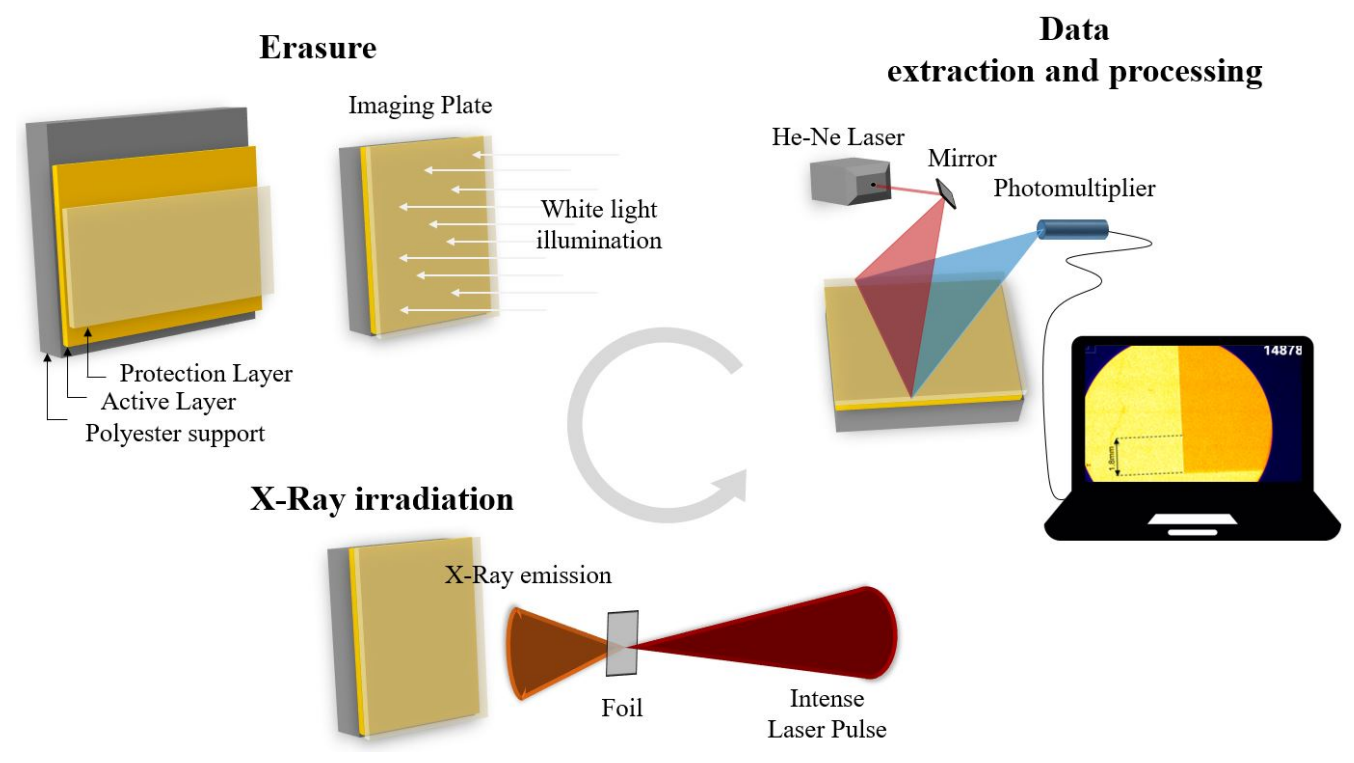

FIGURE IV.5: Imaging plates using steps.

There exists a PSL function to obtain PSL intensity (counts) from the scanner output image grey scale per pixel $G$. Values in PSL are obtained by applying the following conversion formula:

$$
\text { PSL }=\left(\frac{R}{100}\right)^{2}\left(\frac{4000}{S}\right) 10^{L\left[G /\left(2^{B}-1\right)-0.5\right]}
$$

Where $R$ is the scaning pixel size in micrometers, $S$ is the sensitivity setting $(1000,4000,10000)$, $L$ is the latitude $(4,5), B$ is the dynamic range ( 8 or 16 bits), and $G$ is the raw image grayscale value. These parameters can be chosen by the user before scanning the IPs.

It is also noteworthy to mention some of the advantages for using IP's. The most important one consists of their broadband sensitivity to x-rays. IP x-ray sensitivity range is very wide, compared to x-ray ccd cameras, starting at photon energies of few keVs up to almost $100 \mathrm{keV}$. More specifications about of calibrated IP's are found in [Maddox et al., 2011]. In addition, IP's are insensitive to strong electromagnetic pulses, known as EMP in the laser-plasma community, making them very robust compared to x-ray CCD cameras (see next subsection). Moreover, IPs are very easy to use and no electronics are needed for synchronization or acquisition. On the other hand, the main disadvantage of IP's consists of the low repetition rate of use. IPs can only be used for a certain x-ray dose before saturation. Furthermore, the time of scanning and erasing presents on itself a big constraint compared to x-ray CCD cameras. Also, the resolution of IP's is limited by both the crystals emission and scanner resolution. Thus, images obtained with IP's have a maximum resolution of $\sim 25$ microns. This value is twice bigger than the usual pixel size of an x-ray CCD camera. 


\section{IV.2.2 Active detectors: X-ray CCD cameras}

As their name indicates, $x$-ray CCD (Charged Coupled Devices) cameras, are able to operate in the $\mathrm{x}$-ray range. These devices are frequently used in laser-plasma experiments. Used for imaging, x-ray CCD cameras can also be used for pure spectroscopy purposes. The single photon counting mode allows to reconstruct the $\mathrm{x}$-ray spectrum of the source since each pixel intensity value corresponds to an incident photon energy. To operate in the single photon counting mode, the density of incident photons should be much less than the density of pixels. A precise calibration of the camera should be previously carried out before using. Samples of unstable elements emitting specific lines of fixed energy are usually used for this purpose. This operation allows to reconstruct a reliable signal-to-energy function. X-ray CCD cameras chips are mainly made from semiconductor elements ( $\mathrm{Si}$, or $\mathrm{Ge}$ ). These ones are usually used in back-illumination configuration for quantum efficiency optimisation purposes. In contrary to IP's, these sensors are sensitive only to photon energies up to few tens of $\mathrm{keV}$. Another disadvantage of $\mathrm{x}$-ray CCD cameras is their high sensitivity to EMP's. The latter can cause the malfunctioning of the device during the acquisition and can even damage its internal electronics. In addition, one also should be careful using these sensors, especially for laser-driven x-ray sources. Generally, these ones are generated at the same time with high energy charged particles which one should get rid off (using a magnetic dipole for example) to not to damage the camera. On the other hand, x-ray CCD cameras can operate at high repetition rate HRR (very useful for laser facilities operating at HRR) which is mainly due the relatively short readout time (ms) and to the lack of necessity of signal scanning and erasing. Also, pixel sizes of the order of ten microns allows for a higher resolution compared to IP. More details about the x-ray cameras used and applications are given further in the thesis.

\section{IV.3 Testing and validation of the KB microscope}

This section highlights some of the most important results obtained using the adjustable Kirkpatrick-Baez microscope in laser-plasma interaction experiments. Examples of x-ray imaging and transport applications are shown in detail respectively in Sec.IV.3.1 and Sec.IV.3.2. All experiments involving the KB microscope for laser-driven $\mathrm{x}$-ray imaging can be summarised into one common experimental layout shown in Fig.IV.6. The figure consists of three main steps. The first one is the x-ray generation, and depends on the type of laser plasma interaction. The second step is the transport of $\mathrm{x}$-rays, which is fully carried out by the KB microscope. The setting of the latter should be adjusted depending of the purpose of each experiment. The third and last step is the x-ray detection. This one can be carried out via an $\mathrm{x}$-ray detector such $\mathrm{x}$-ray cameras or Imaging Plates.

\section{IV.3.1 The KB microscope for $x-$ ray imaging}

\section{IV.3.1.a Proton acceleration experiment 1 (L.Volpe 2018)}

We are presenting here the results obtained using the adjustable KB microscope for a commissioning experiment on VEGA-II which targeted the study of proton acceleration, previously introduced in Sec.I.2.7. The KB microscope has been implemented in CLPU's target area to image 

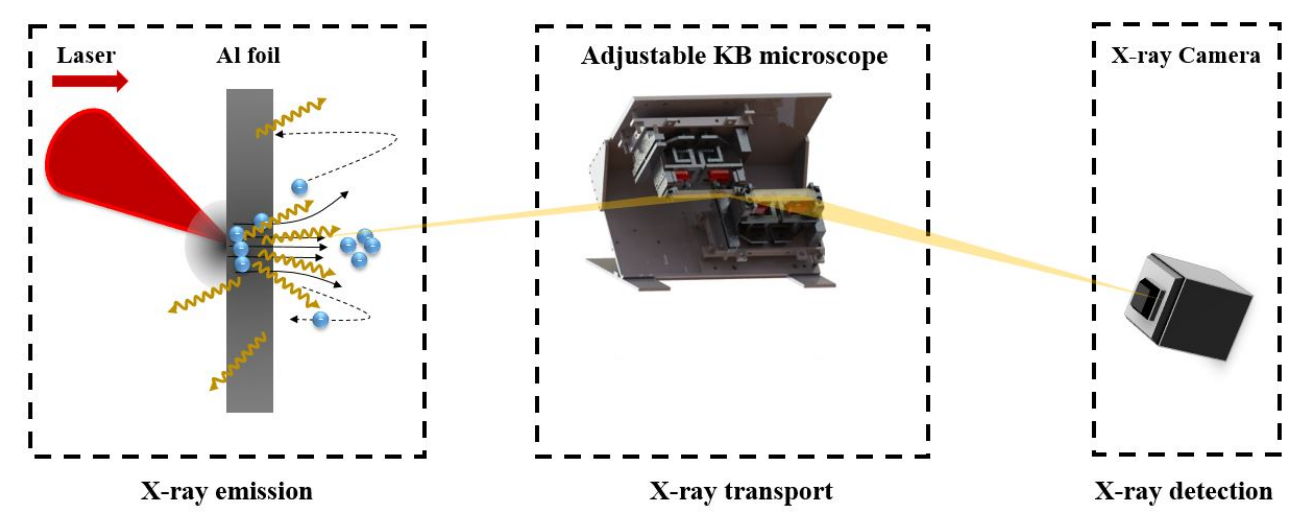

FIGURE IV.6: General setup for X-ray imaging using the adjustable KB microscope.

the VEGA-II driven K- $\alpha$ emission line of aluminium generated by the interaction of laser accelerated hot electrons inside the target itself $(6 \mu \mathrm{m}$ tick Al target). The theoretical intensity on target was of the order of $2 \times 10^{20} \mathrm{~W} . \mathrm{cm}^{-2}$ and the electron energy was estimated to be of the order of $1.2 \mathrm{MeV}$. My responsibility during this experiment was to build the experimental setup and to align the laser system. I was fully responsible for the installation of the x-ray transport line using the KB microscope. Carrying out data acquisition and analysis was also a large part of my task. The 3D scheme showing my part of the experimental setup is given by Fig.IV.7.

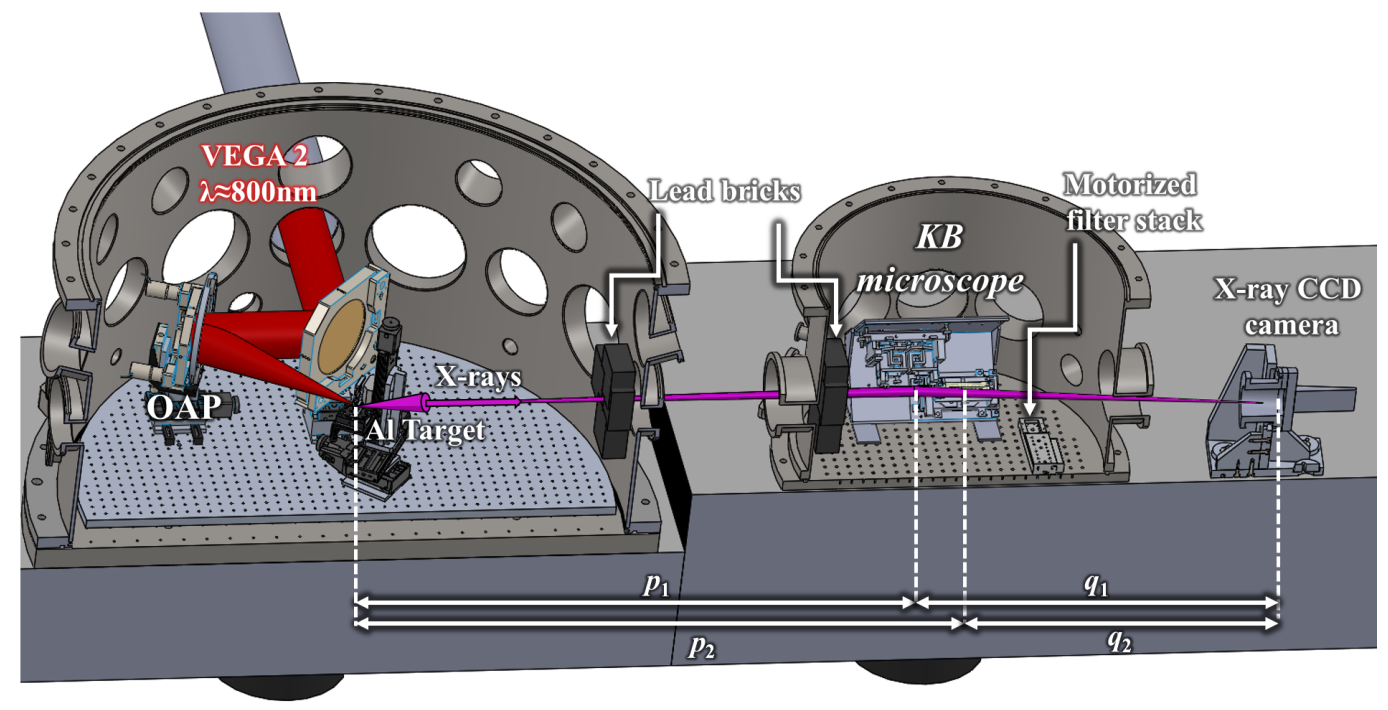

FIGURE IV.7: Experimental setup for the first proton acceleration experiment 1 carried out in CLPU.

The generated x-ray radiation was collected by an x-ray CCD camera (Greateyes model GE 1024 $256 B I D D$ ) with $26 \mu \mathrm{m}$ pixel size. The chip of the camera was covered with two layers of $2 \mu \mathrm{m}$ thick Mylar. The latter were coated with a thin 100nm layer of aluminium to block the visible light. The KB microscope was placed at $1.2 \mathrm{~m}$ (i.e. $p_{1}=1.2 \mathrm{~m}$ ) away from the the target center at 
$30^{\circ}$ with respect to the target normal. We note that the device was placed and adjusted to image the x-ray source exactly onto the x-ray camera (chip) plane. A motorised filter stack containing $\mathrm{Al}$ foils of different thickness was also placed in the middle of the $\mathrm{x}$-ray transport line for both intensity and spectral filtering purposes.The figures IV.8 and IV.9 show the x-ray transmission of the filters used in front of the x-ray ccd chip in linear and log scales. A total of $6 \mu \mathrm{m} \mathrm{Al}$ and the 2 layers of $2 \mu \mathrm{m}$ aluminized Mylar were used between the source and the $\mathrm{x}$-ray camera chip.

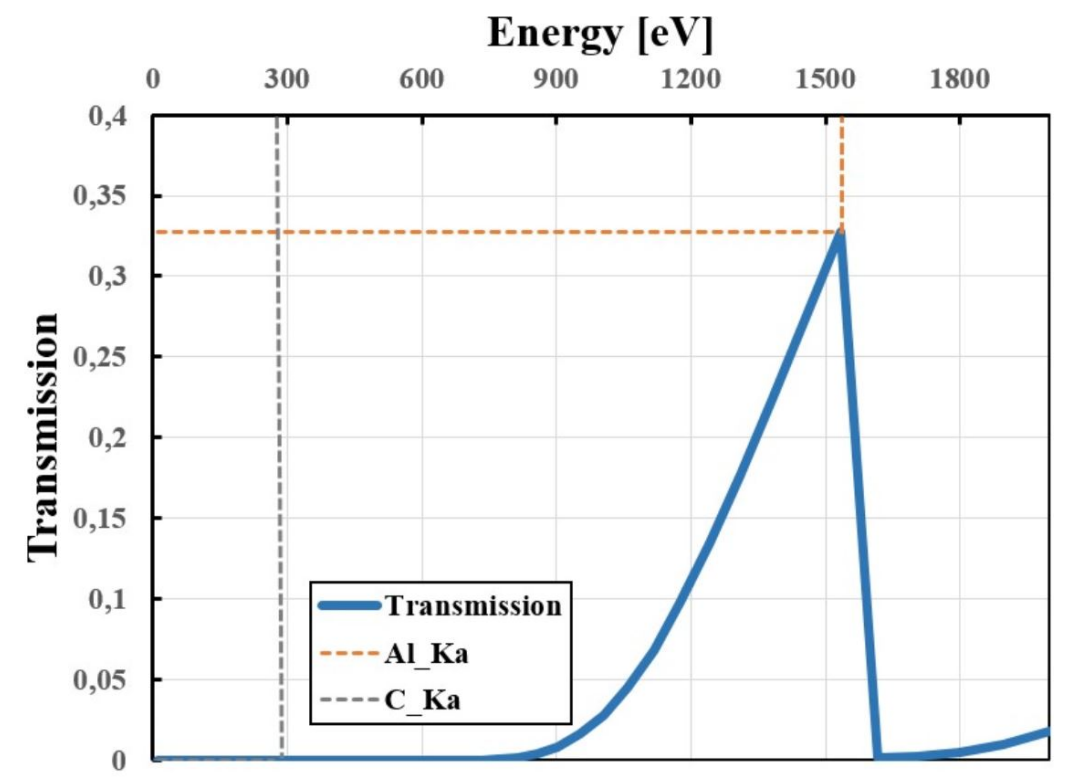

FIGURE IV.8: Filter transmission for $\mathrm{C}-\mathrm{K} \alpha(277 \mathrm{eV})$ and $\mathrm{Al}-\mathrm{K} \alpha$ for a set of filters of $2 \times 2 \mu \mathrm{m}$ aluminized Mylar and $1 \times 6 \mu \mathrm{m}$ aluminium.

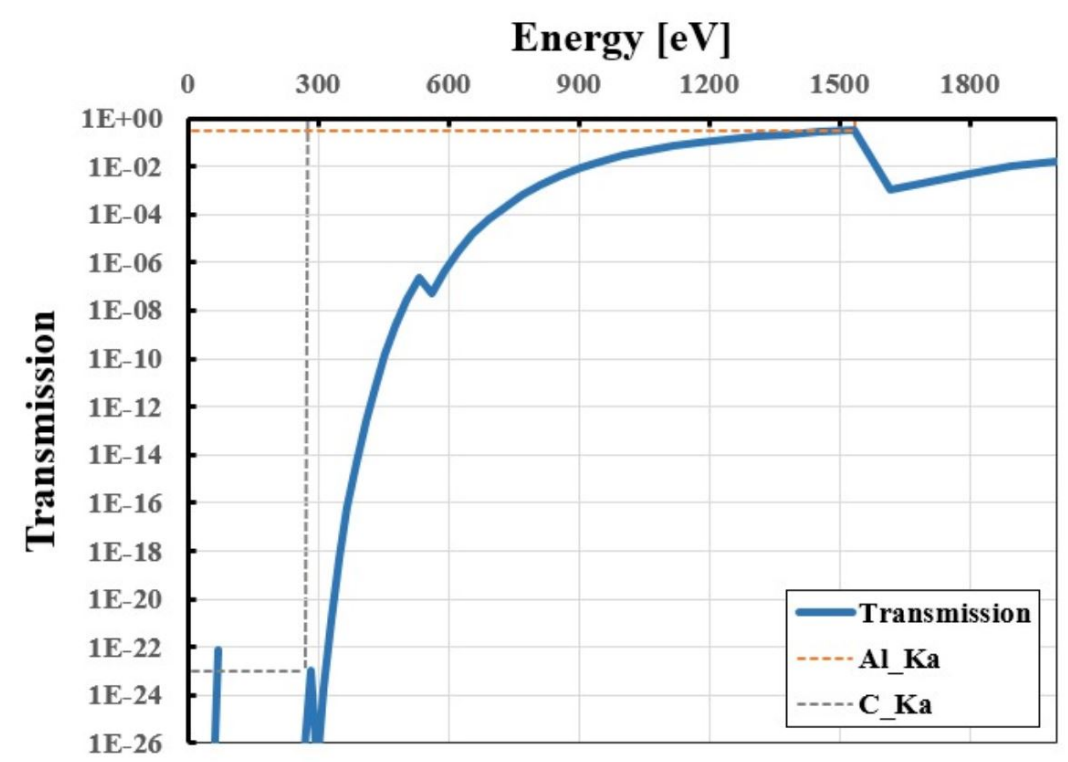

FIGURE IV.9: The same curve of Fig.IV.8 with a logarithmic scale.

According to Fig.IV.9, it is clearly seen that the low energy x-rays are strongly attenuated meanwhile the transmission is maximum for the $\mathrm{Al}-\mathrm{K} \alpha$ emission line (due to the high transmission of 
the K-edge range). This set of filters is then acting like a high pass filter for Al-K $\alpha$ emission line. It is noteworthy to remind that the KB mirrors can act as a cut-off filter for higher energy $\mathrm{x}$-ray photons, which is mainly due to the relatively low reflectivity for this region of the spectrum. Using a stack of filters turned out to be very important during the $\mathrm{x}$-ray focusing sequence, since a shot to shot adjusting method has been carried out for the optimisation of the KB x-ray spot (see Fig.IV.10). This technique consists of a gradual bending of the mirrors. In the beginning of the focusing, the $\mathrm{x}$-ray spot is less intense, and so, only high transmission filters are needed. While carrying out focusing, the created 2D KB microscope image becomes more and more intense, which then requires the use of thicker foils (lower transmission filters). Motorising the $\mathrm{x}$-ray filters turned out to be very practical, since all the steps are carried out in vacuum. The necessity of changing the filters gradually can be clearly seen in Fig.IV.10.
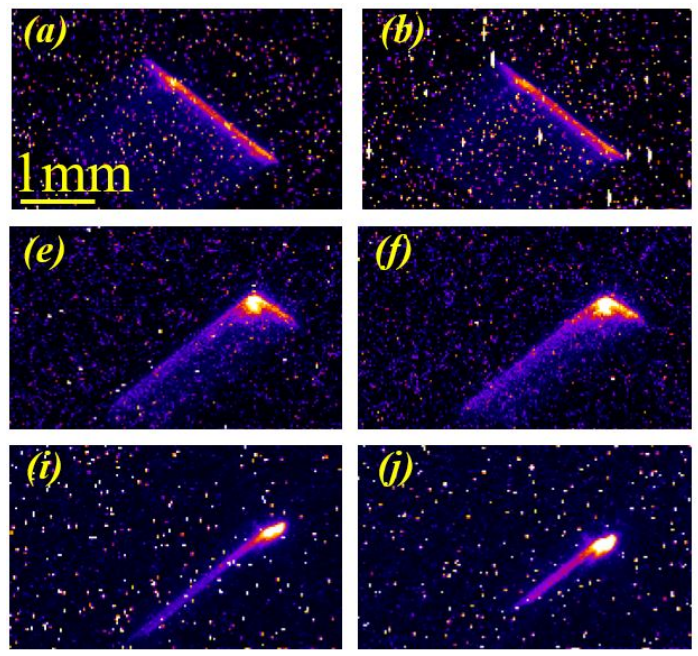
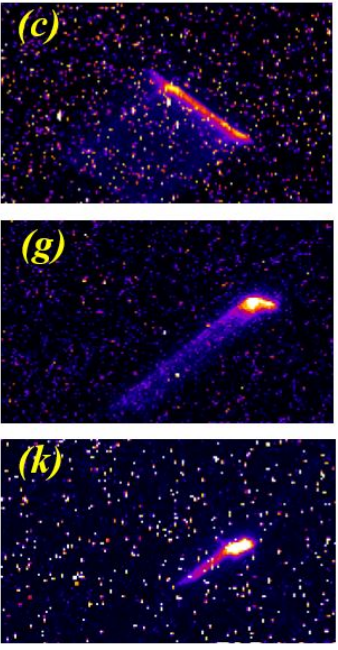
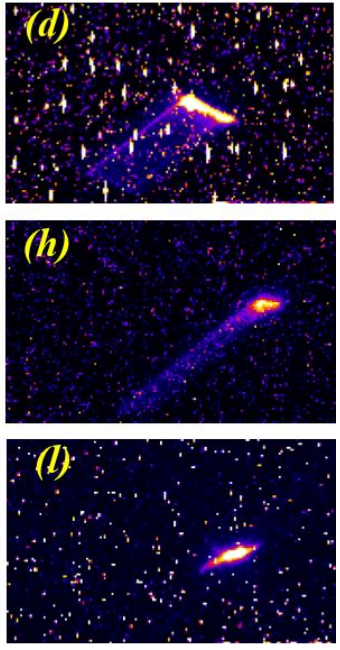

FIGURE IV.10: Images of the x-ray CCD camera at different focusing stages of the KB microscope.

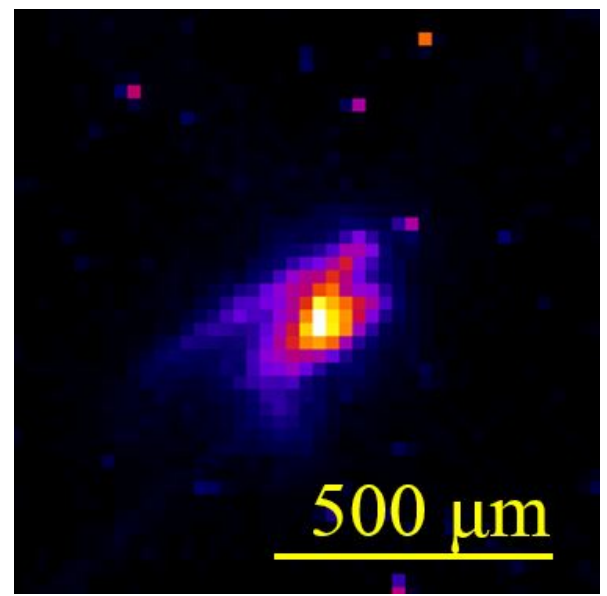

FIGURE IV.11: The optimised KB microscope focal spot after adjustments.

Fig.IV.10 highlights the effects of step by step bending of the KB mirrors on shaping the Al-K $\alpha$ radiation spot (always in shot to shot regime). The independent effect of adjusting each single 
mirror on focusing the beam in each transverse dimension is also clearly identified. The upper eight $\mathrm{x}$-ray images of Fig.IV.10 (a-h) correspond to the focus adjustment carried out with the first bender, while the bottom ones (i-1) correspond to the effect of the other bender. The final measured x-ray spot size, seen in Fig.IV.11, was $134.2 \mu \mathrm{m}$ by $99.6 \mu \mathrm{m}$ for the mirrors 1 and 2 respectively. We remind that the magnification was $M_{1} \sim 0.66$ for mirror 1 and $M_{2} \sim 0.54$ for mirror 2, which means that the real size of the source was equal to $\sim 203 \mu \mathrm{m}$ in dimension 1 and to $\sim 184 \mu \mathrm{m}$ in the perpendicular dimension. We also note that the error on these measurements is mainly due to the big pixel size of the camera $( \pm 26 \mu \mathrm{m})$. It is also noteworthy to add that the final image obtained is a superposition of the $\mathrm{K}-\alpha$ source at the laser focal spot position and the $\mathrm{K}-\alpha$ radiation coming from the hot electron refluxing effect, inside the target itself. We note that similar values of mirror slope error of Fig.III.13 would lead to a spot size of the order of 50 to $100 \mu \mathrm{m}$. On the other hand, according to Fig.III.16, it is clear that surface aberrations are significantly less than $100 \mu \mathrm{m}$.

Finally, we have also carried out another study aiming to measure the resolution of the KB imager. For that, we redid the same experiment but this time, placing an x-ray back-lighted metallic grid of $100 \mu \mathrm{m}$ wires separated by free spaces of $110 \mu \mathrm{m}$ (always as the same $\mathrm{x}$-ray path than before). The result of the x-ray radiography projection is shown in Fig.IV.12. We can
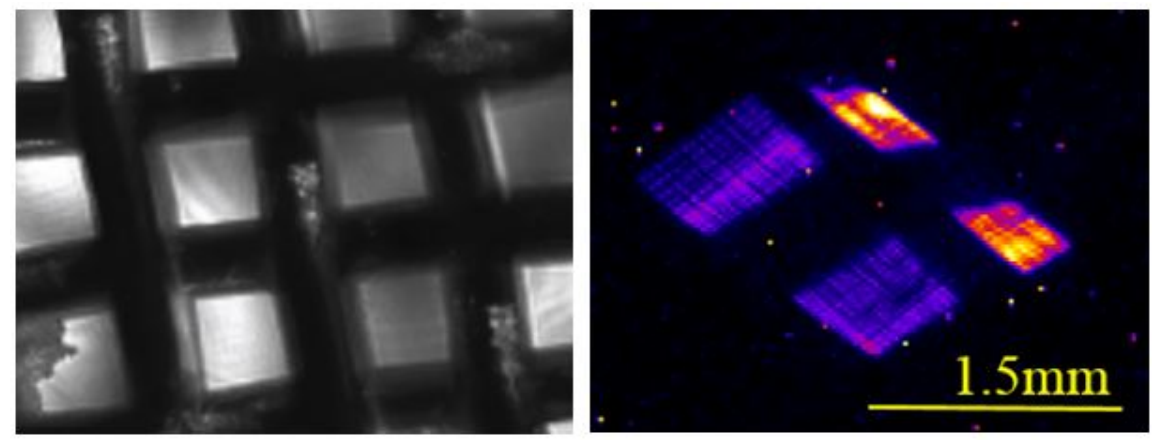

FIGURE IV.12: (left) Image of the metallic grid $(100 \mu \mathrm{m}$ by $100 \mu \mathrm{m}$ wires) used for the $\mathrm{x}$-ray radiography. (Right) $\mathrm{X}$-ray projection radiography of the same metallic grid.

see from Fig.IV.12 (left) that the image imperfections of the x-ray radiography are mainly due to the manufacturing of the grid. In addition to this, the optical aberrations introduced by the $\mathrm{KB}$ microscope are also responsible of the image deformation. These convolution of aberrations made the calculation of resolution impossible, so, no concluding quantitative results have been extracted. We point out that the actual $\mathrm{KB}$ microscope has been used in actual laser-plasma experiments and two scientific articles have successfully been published on the results thus far, as can be found in [Volpe et al., 2019] and [Zeraouli et al., 2019].

\section{IV.3.1.b Strong shocks generation experiment 1 (D. Batani 2018)}

The adjustable KB microscope has also been used in another experiment in CLPU (see Fig.IV.13) where the experimental setup was similar to the one of proton acceleration experiment 1 with only one difference, concerning the magnification distances of the $\mathrm{KB}$ microscope, presented in details, in Fig.IV.14. From one side, the goal of strong shocks generation experiment 1 was to study the generation of strong shocks in matter using ultra-intense laser pulses (using VEGA-II, 
$6 \mathrm{~J}, 30 \mathrm{fs})$ with an intensity on targets of the order of $2 \times 10^{20} \mathrm{~W} \cdot \mathrm{cm}^{-2}$. The hot electron energy was estimated to be of the order of $1.2 \mathrm{MeV}$. On the other side, the main purpose of using the KB was to image the $\mathrm{K} \alpha$ signal emitted from the high energy electrons transport in targets of different thickness. I have been responsible for both, the installation of the experimental setup together with data acquisition and analysis.

The target holder shown in Fig.IV.15 and used for strong shocks generation experiment 1 (previously used in proton acceleration experiment 1) consists of two aluminium plates with two sets of $8 \times 8$ holes each, placed in a coaxial configuration. The rear side of the holder was machined into a conical shape in order to increase the solid angle seen from the back. This wide viewing angle increases the possibility of installation of more diagnostics. This target holder allowed for a total shot sequence of 128 shots, making it very practical for high repetition rate experiments (avoiding to break vacuum for changing targets). In addition, this target holder was also designed to hold targets of different type and thickness which result to be very practical for keeping the same laser and vacuum conditions during a sequence of shots.

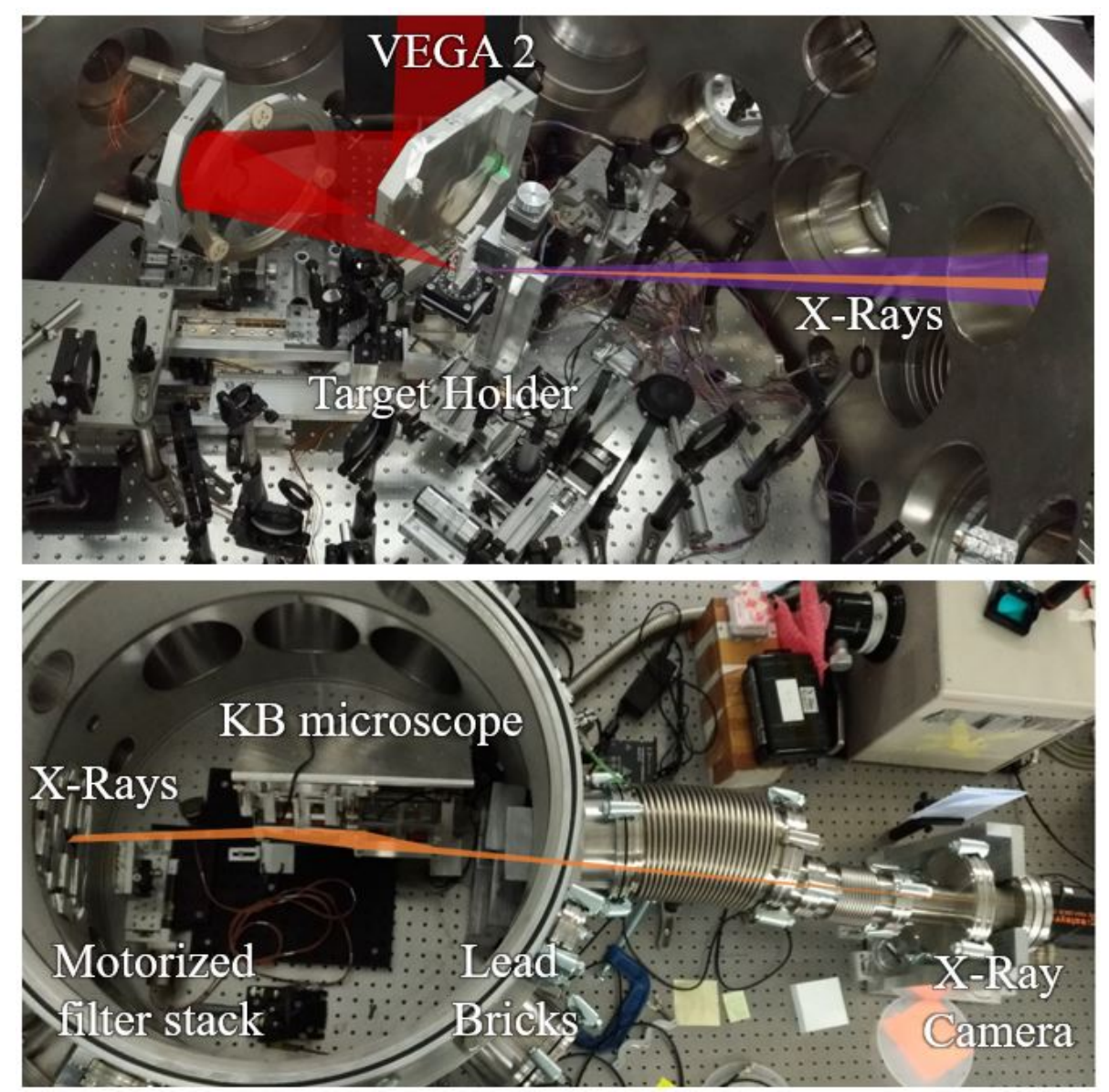

FIGURE IV.13: Picture of the experimental setup for strong shocks generation experiment 1 . The main interaction chamber for $\mathrm{x}$-ray generation (top). X-ray transport and focusing (bottom).

We can see clearly from Fig.IV.14 that the KB microscope is used in a demagnification configuration. $M_{\mathrm{A}}$ and $M_{\mathrm{B}}$, respectively the magnification for mirror $\mathrm{A}$ and mirror $\mathrm{B}$, were $\sim 0.87$ and 


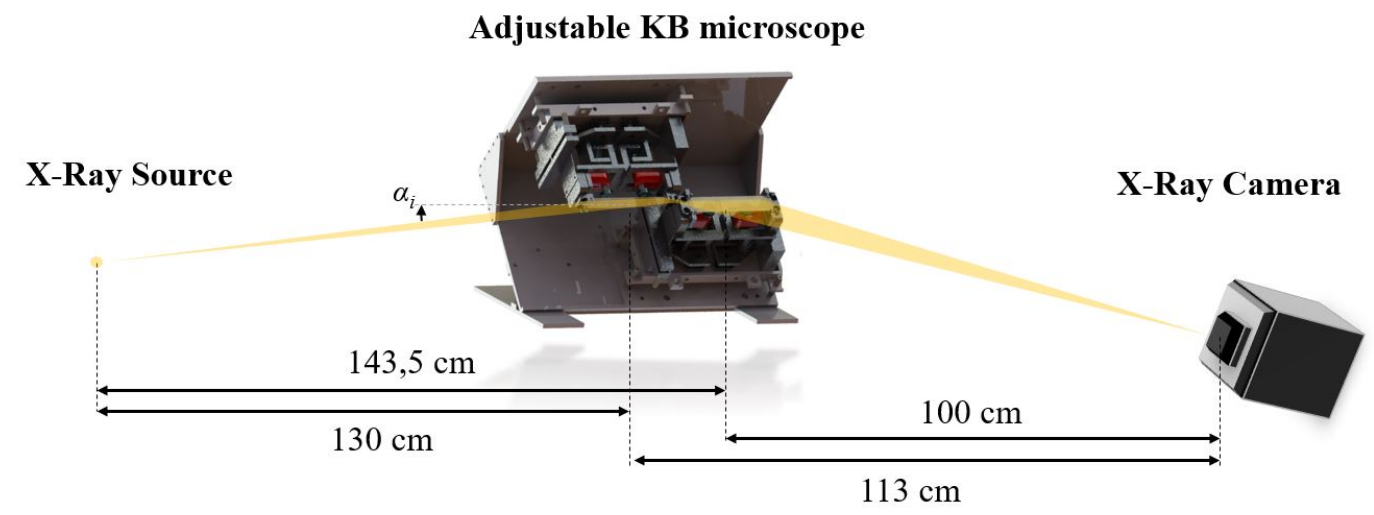

FIGURE IV.14: Scheme for the KB parameters used in proton acceleration experiment 2. According to reflectivity and acceptance simulations in Fig.III.8, the grazing incidence angle on both mirrors $\alpha_{i}$ was fixed at $34 \mathrm{mrad}$.

\section{Front Side}

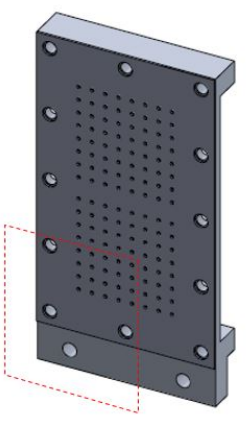

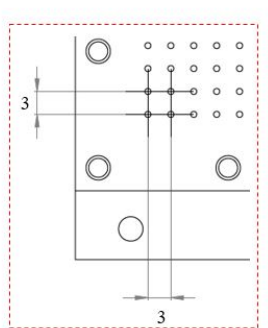

\section{Back Side}

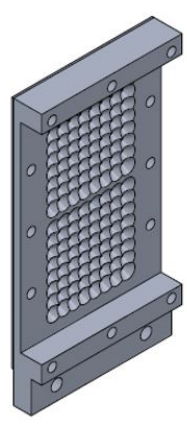

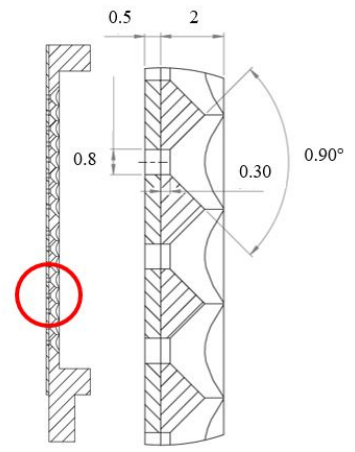

FIGURE IV.15: Target holder used for both, proton acceleration experiments 1 and strong shocks generation experiment 1 . The distances are given in millimetres.

$\sim 0.70$ respectively. The targets used in this experiment were all made from aluminium of 70 , 50 and 30 microns thickness. In some shots, thinner targets of $10 \mu \mathrm{m}$ aluminium with a $2 \mu \mathrm{m}$ titanium tracer layer were also used. A curved Bragg crystal was also placed in this experiment for the same purpose as the KB microscope. In this case, it was aligned to reflect the Ti-K $\alpha$ emitted from the $2 \mu \mathrm{m}$ titanium tracer layer. The idea was to extract and cross-correlate the data from both diagnostics (Bragg imager and $\mathrm{KB}$ ) for results comparative purposes. Unfortunately, the Bragg crystal didn't produce any useful images. Thus, no data could be extracted for the Bragg crystal imager. On the other hand, the KB microscope worked successfully and examples of data obtained for different targets are shown in Fig.IV.16.

The generated x-rays were collected by the same x-ray CCD camera used in proton acceleration experiment 1 (Greateyes model GE 1024256 BI DD with $26 \mu \mathrm{m}$ pixel size). In addition, the chip of the camera was covered with two layers of $2 \mu \mathrm{m}$ thick Mylar (each Mylar layer is coated with a thin $100 \mathrm{~nm}$ layer of aluminium to block the visible light). It is also noteworthy to mention that a motorized filter stack has been installed in the way of $\mathrm{x}$-rays such that only the Al-K $\alpha$ signal is filtered. The filter used to obtain the data of Fig.IV.16 was a $3 \mu \mathrm{m}$ thick layer of Al. The curve of x-ray transmission of the 3 microns filter is similar to the one of Fig.IV.8 and Fig.IV.9 multiplied 

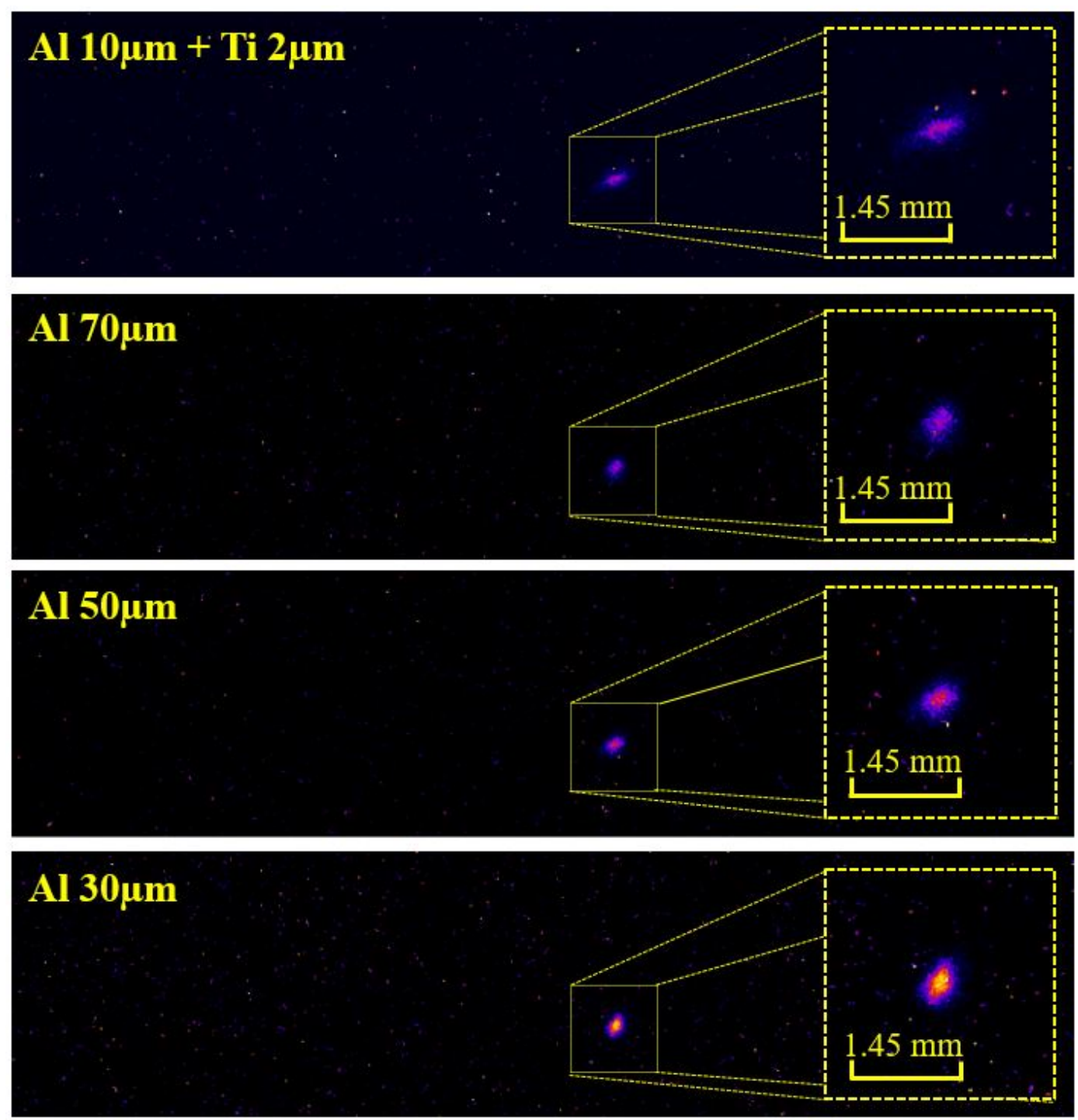

FIGURE IV.16: Experimental data obtained with the adjustable KB for targets of different thickness. These data were collected with the x-ray camera of $26 \mu \mathrm{m}$ pixel size. The larger images show the total area of the camera chip.

by a factor of two.

Line-out profiling of the data shown in Fig.IV.16 has been carried out for each shot and along each mirror direction for single shots. The results of this operation are shown in Fig.IV.17.

A comparison between the fits of the data shown in Fig.IV.17 for each direction is presented in Fig.IV.18

Taking into account the magnification in each direction $M_{A}$ and $M_{B}$, respectively the magnification in direction $\mathrm{A}$ and $\mathrm{B}$, the final real $\mathrm{Al}-\mathrm{K} \alpha$ spot size are shown in the table of Fig.IV.19.

For more accuracy, an average taken over ten shots for each different target have been calculated to characterize the KB spot size for each bender. An averaging over ten shots of the $\mathrm{x}$-ray 

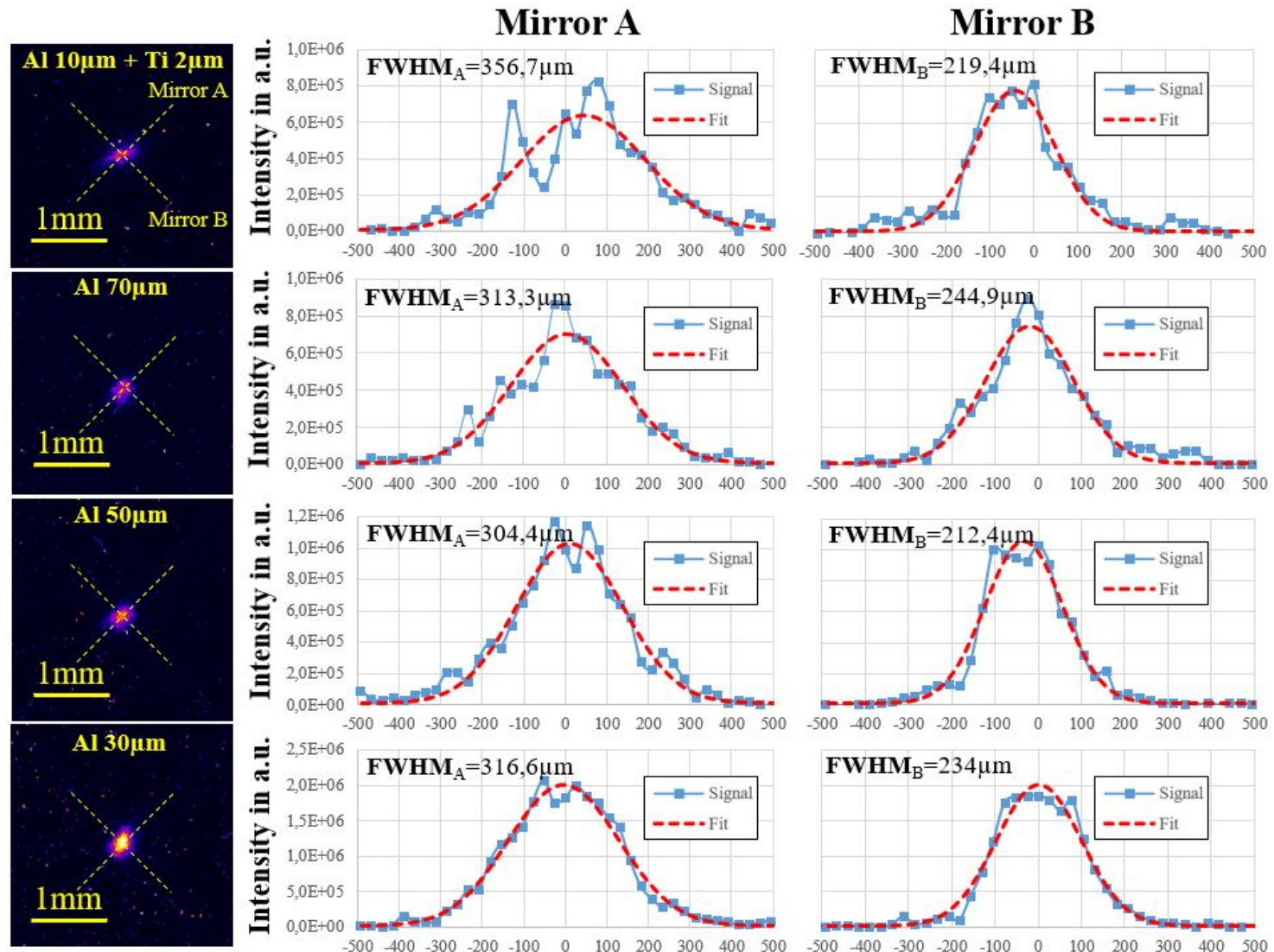

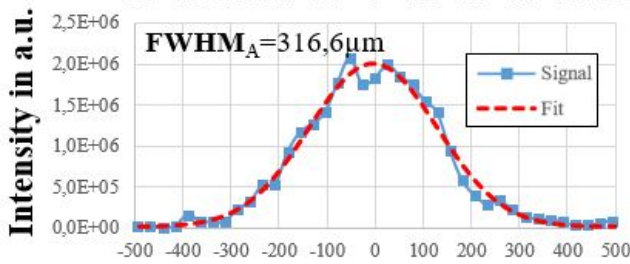

Distance in $[\mu \mathrm{m}]$

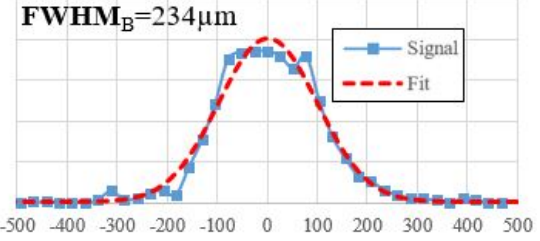

Distance in $[\mu \mathrm{m}]$

FIGURE IV.17: Single shot profile line-outs for different target types.
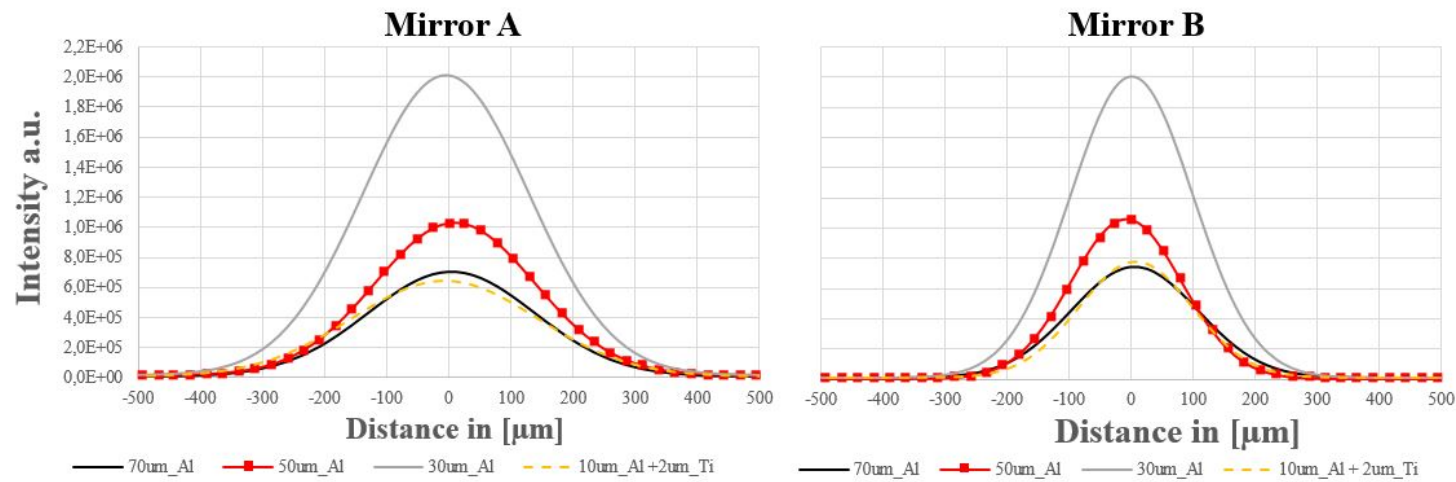

FIGURE IV.18: Comparison between the signals fit of Al-K $\alpha$ spot sizes for each target type for the two focusing directions.

intensity in focus has also been measured for each targets. The summary of these measurements are shown in Fig.IV.20.

According to Fig.IV.17, Fig.IV.19 and Fig.IV.20, we can see clearly how the spot intensity decreases from thin to thicker targets. This effect is mainly due to the absorption of the electrons in the targets (due to the matter stopping power). The thicker is the material and more is the 


\begin{tabular}{|c|c|c|c|c|}
\hline & \multicolumn{4}{|c|}{ Target type } \\
\hline & $\begin{array}{l}10 \mu \mathrm{m} \mathrm{Al} \\
+2 \mu \mathrm{m} \mathrm{Ti}\end{array}$ & $70 \mu \mathrm{m} \mathrm{Al}$ & $50 \mu \mathrm{m} \mathrm{Al}$ & $30 \mu \mathrm{m} \mathrm{Al}$ \\
\hline $\begin{array}{c}\text { Size in direction } \mathrm{A} \\
{[\mu \mathrm{m}]}\end{array}$ & $414,8 \pm 59,7$ & $364,3 \pm 59,7$ & $354 \pm 59,7$ & $368,2 \pm 59,7$ \\
\hline $\begin{array}{c}\text { Size in direction B } \\
{[\mu \mathrm{m}]}\end{array}$ & $318 \pm 74,2$ & $354,9 \pm 74,2$ & $307,8 \pm 74,2$ & $339,1 \pm 74,2$ \\
\hline $\begin{array}{c}\text { Average } \\
\text { dimension }[\mu \mathrm{m}]\end{array}$ & $366,4 \pm 66,9$ & $359,6 \pm 66,9$ & $330,9 \pm 66,9$ & $353,6 \pm 66,9$ \\
\hline
\end{tabular}

FIGURE IV.19: Real Al-K $\alpha$ spot sizes for different target types for one single shot.

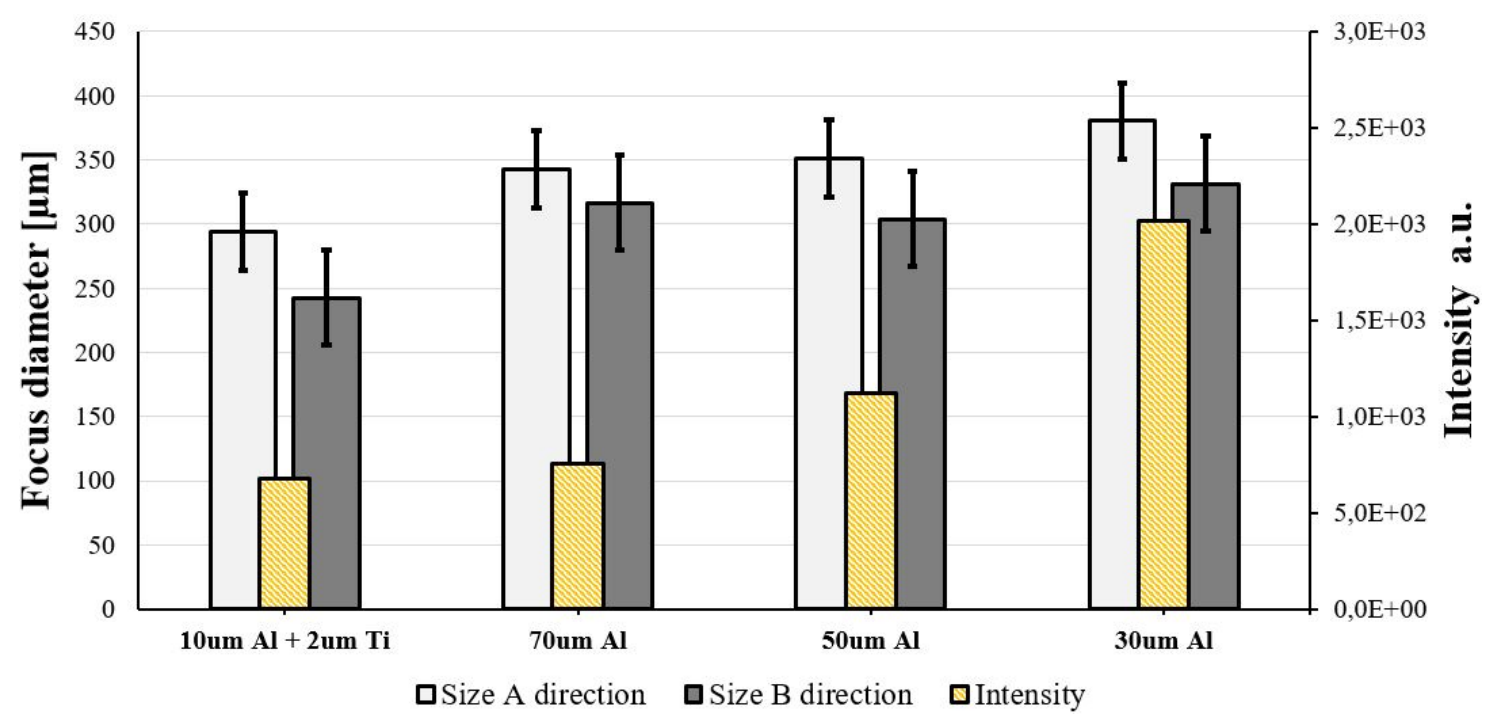

FIGURE IV.20: KB spot characterisation: $x$-ray spot size (for each bender) and relative intensity for different target types.

absorption (for both photons and electrons). The case of $\mathrm{Al} 10 \mu \mathrm{m}+\mathrm{Ti} 2 \mu \mathrm{m}$ is different from the other ones since the Ti layer, previously supposed to perform as a tracer for the Bragg crystal is actually acting like a $\mathrm{K} \alpha$ dumper from the $\mathrm{KB}$ side of view (since it is stopping $83 \%$ of this aluminium emission line [CXRO, 2019]).

The other information we can deduce from the graph of Fig.IV.20 is how the x-ray spot changes in size for thicker targets. Using the KB in this experiment allows us then to study both electron absorption and refluxing [Volpe et al., 2013, Sentoku et al., 2003, Neumayer et al., 2010] for different material thickness.

According the consistent results shown in Fig.IV.20 we can conclude that the KB microscope is a reliable and robust tool for alignment and imaging in different configurations making it one of the reference diagnostics in many experiment at CLPU. It is noteworthy to add that, even misaligned (signal on the camera but with strong optical aberrations), the KB microscope can be used as an $\mathrm{X}$-ray collector playing the role of an on-line diagnostic. In other words, one can use the $\mathrm{KB}$ signal to define a criteria for qualitative characterisation of the shot (more laser absorption generates more $\mathrm{x}$-rays, thus more signal is transported by the $\mathrm{KB}$ and so a stronger intensity signal is observed on the $\mathrm{x}$-ray detector). This criteria was used during many experiments to see 
how precise we were hitting the targets since the results were given instantaneously thanks to the use of x-ray cameras.

\section{IV.3.1.c Proton acceleration experiment 2 (R. Fedosejevs 2019): KB microscope versus curved Bragg crystal}

This subsection highlights some differences between using the adjustable KB microscope and a spherical Bragg crystal. We are presenting the results obtained from a spherical Bragg imager used in proton acceleration experiment 2 at CLPU facility where the experimental conditions were almost similar to the ones shown in proton acceleration experiments 1 and strong shocks generation experiment 1 (using VEGA-II laser at full power, 6J, 30fs duration) with an intensity on target of the order of $2 \times 10^{20} \mathrm{~W} . \mathrm{cm}^{-2}$. The goal of the experiment was to accelerate particles in solid targets using TNSA mechanism. The targets used were made of $10 \mu \mathrm{m}$ aluminium foils covered in the rear side with a cooper tracer layer of $25 \mu \mathrm{m}$ thickness. A spherical curved bragg crystal, made of quartz, was used to image the $\mathrm{Cu}-\mathrm{K} \alpha$ emission line generated by the high energy electron transport in matter. The experimental setup carried out for proton acceleration experiment 2 is shown in Fig.IV.21. I have participated to laser alignment, to the installation of the experimental setup and also to both acquisition and analysis of the data.

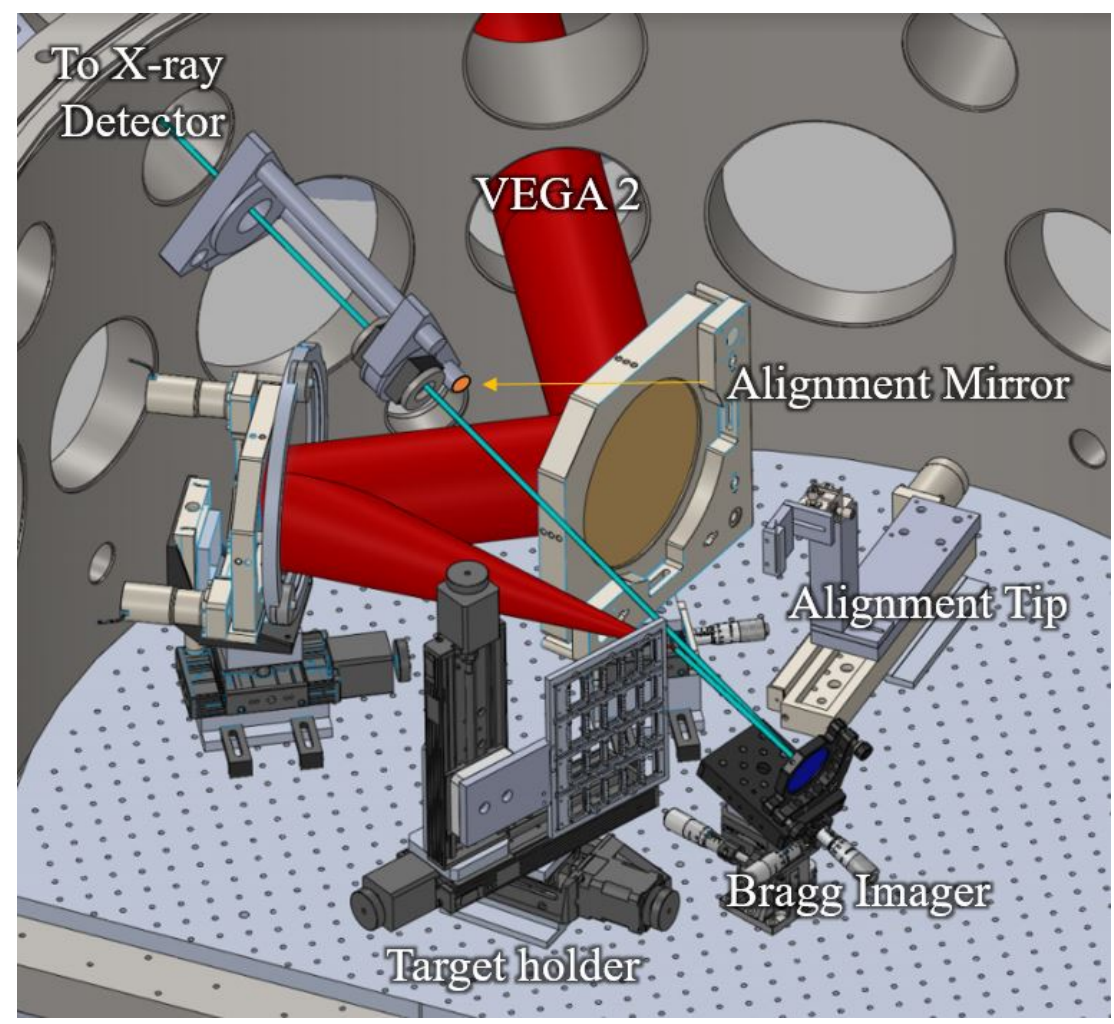

FIGURE IV.21: Proton acceleration experiment 2 setup for $\mathrm{x}$-ray imaging.

Aligning spherical Bragg crystals for imaging purposes is very challenging. With a fixed radii of curvature, one is obliged to install them with high accuracy. Precise geometrical calculations needs to be carried out before the installation. Theoretically, Fig.IV.22 represents one of the most 
accurate schemes we used for curved Bragg crystal alignment, consisting of defining angles by calculating the distances between the optics. Step 0 before the experiment is to define which radiation to transport. The user can then choose the adequate crystal with an optimised $2 \mathrm{~d}$ interplanar spacing and also with the right radii of curvature $\mathrm{R}_{c}$.

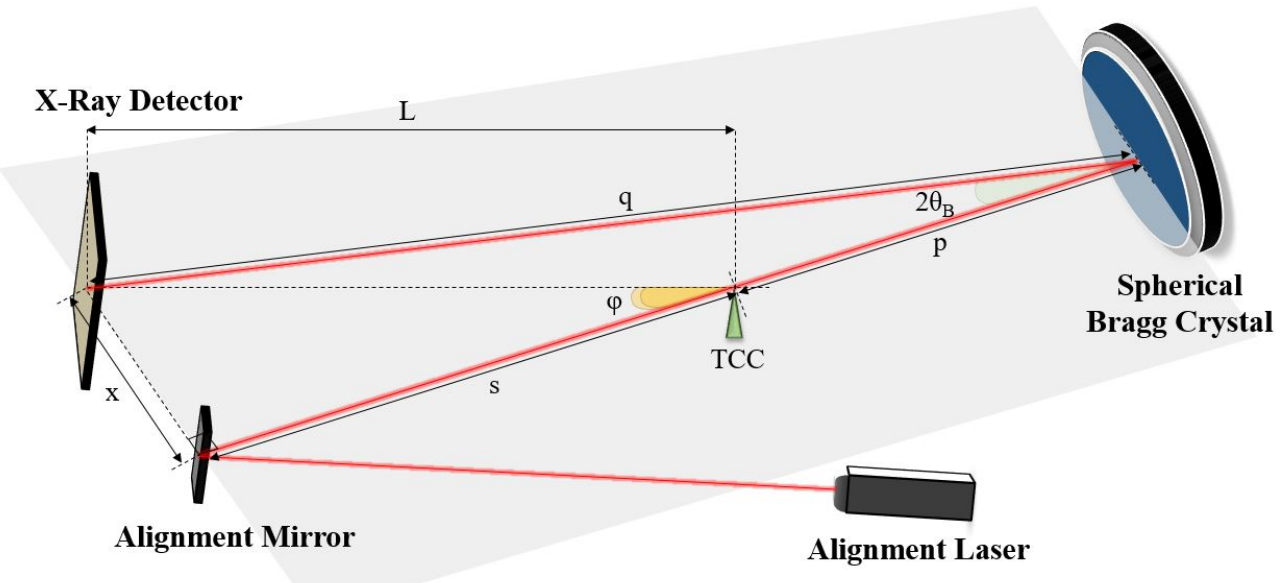

FIGURE IV.22: Parameters definition for aligning spherical Bragg crystal.

From one side, the first fundamental equation ruling the $\mathrm{x}$-ray imaging is given by

$$
\frac{1}{\mathrm{p}}+\frac{1}{\mathrm{q}}=\frac{2}{\mathrm{R}_{c}}
$$

Also, according to Fig.IV.22 we can write

$$
\begin{aligned}
& \mathrm{x}=\mathrm{q} \sin \left(2 \theta_{B}\right) \\
& \mathrm{x}=\mathrm{L} \sin (\varphi)
\end{aligned}
$$

We can write then $\varphi$, the angle between the alignment mirror to TCC and the x-ray detector to TCC as

$$
\varphi=\operatorname{asin}\left(\frac{\mathrm{q}}{\mathrm{L}} \sin \left(2 \theta_{B}\right)\right)
$$

From Fig.IV.22 we can also define, s, as the distance between the alignment mirror to TCC as

$$
\mathrm{s}=\mathrm{q} \cos \left(2 \theta_{B}\right)-\mathrm{p}
$$

Using Eq.IV.3 and Eq.IV.6 one can write the expression of $\mathrm{L}^{2}$ where

$$
\mathrm{L}^{2}=\mathrm{x}^{2}+\mathrm{s}^{2}
$$

We obtain then the second fundamental expression relating $\mathrm{L}, \mathrm{p}, \mathrm{q}$ and $\theta_{B}$ written as

$$
\mathrm{L}^{2}=\mathrm{p}^{2}+\mathrm{q}^{2}+2 \mathrm{pq} \cos \left(2 \theta_{B}\right)
$$

One can use the defined equations to carry out macroscopic rough alignments. Small adjustments 
can then be made using the optomechanical screws to position the image on the centre of the $\mathrm{x}$ ray detector. For more accuracy, it is preferable to use a camera during the alignment before exchanging it with an $\mathrm{x}$-ray detector.

The experimental data were collected using an Andor IKON-M x-ray CCD camera. The camera chip was made by a matrix of $1024 \times 1024$ pixels of $13 \mu \mathrm{m}$ size. A spherically bent quartz 211 crystal $(2 \mathrm{~d}=3.082)$ was used to image the $\mathrm{Cu}-\mathrm{K} \alpha$ emission line. The crystal reflectance curve can be simulated using the XOP program and is given by FigIV.23.

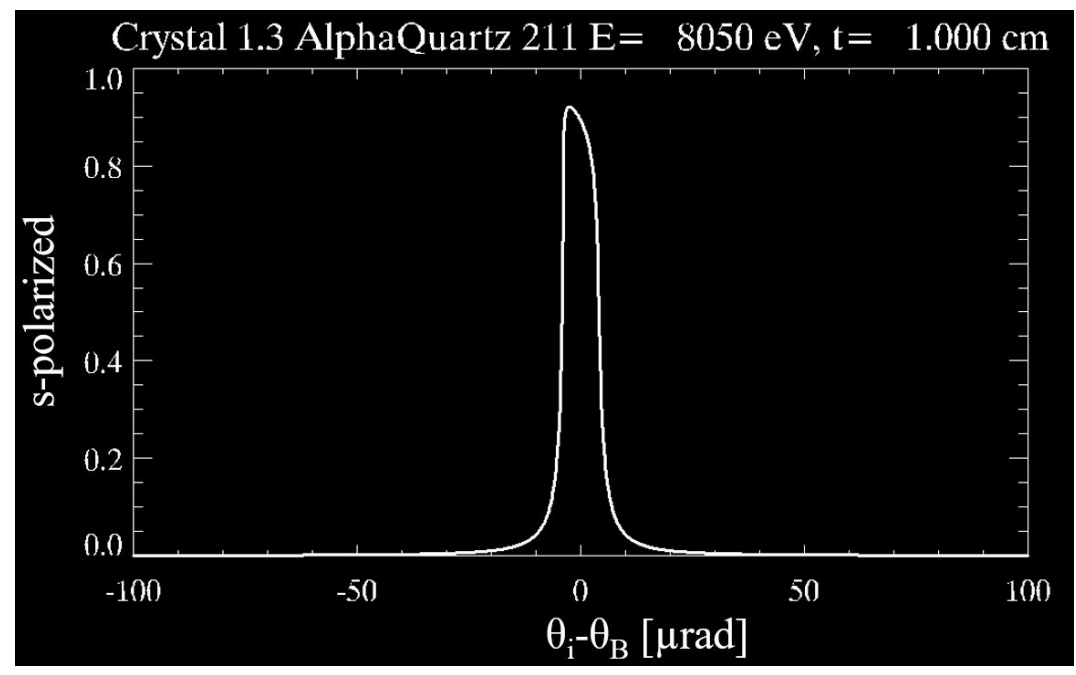

FIGURE IV.23: Reflectance of an alpha quartz crystal for $\mathrm{Cu}-\mathrm{k} \alpha$ arround the Bragg angle. The curved here corresponds to a flat crystal, and not to a curved one, where latter is more asymmetrical (similar example in [Antonelli et al., 2015]).

The crystal's radii of curvature, $\mathrm{R}_{c}$, was equal to $25 \mathrm{~mm}$ and was placed at a distance $\mathrm{p}=17.5 \mathrm{~cm}$ from the target and at $\mathrm{q}=94.5$, the distance between the crystal to the $\mathrm{x}$-ray $\mathrm{CCD}$. According to these numbers the magnification of this system is equal to $M=5.4$. The signal to noise ratio (SNR) was relatively low (SNR 1.2), thus, in order to distinguish better the signal from background, a sum of multiple shots was carried out. Fig IV.24 shows an example of the data acquired for 1 shot and a sum of 10 shots on the left to the right, respectively.

The x-ray spot profiles (calculated) obtained for a sum of 10 shots (in both horizontal and vertical dimension) are shown in Fig.IV.25

According to Fig.IV.25, the x-ray spot is symmetrical and has the same size in both dimensions. The final calculated $\mathrm{Cu}-\mathrm{K} \alpha$ spot size at FWHM is close to $\sim 110 \mu \mathrm{m}$.

Fig.IV.26 is summarising the results of all the experiments (proton acceleration experiments 1, 2 and strong shocks generation experiment 1 ) in a more schematic way for an easy data comparison. We note that the size scales were respected for drawing the $\mathrm{K} \alpha$ spot size, $\mathrm{K} \alpha \mathrm{SNR}$ and target thickness.

A quantitative data comparison between both, the KB microscope and the curved Bragg crystal, for different targets is presented in the table of Fig.IV.27. 

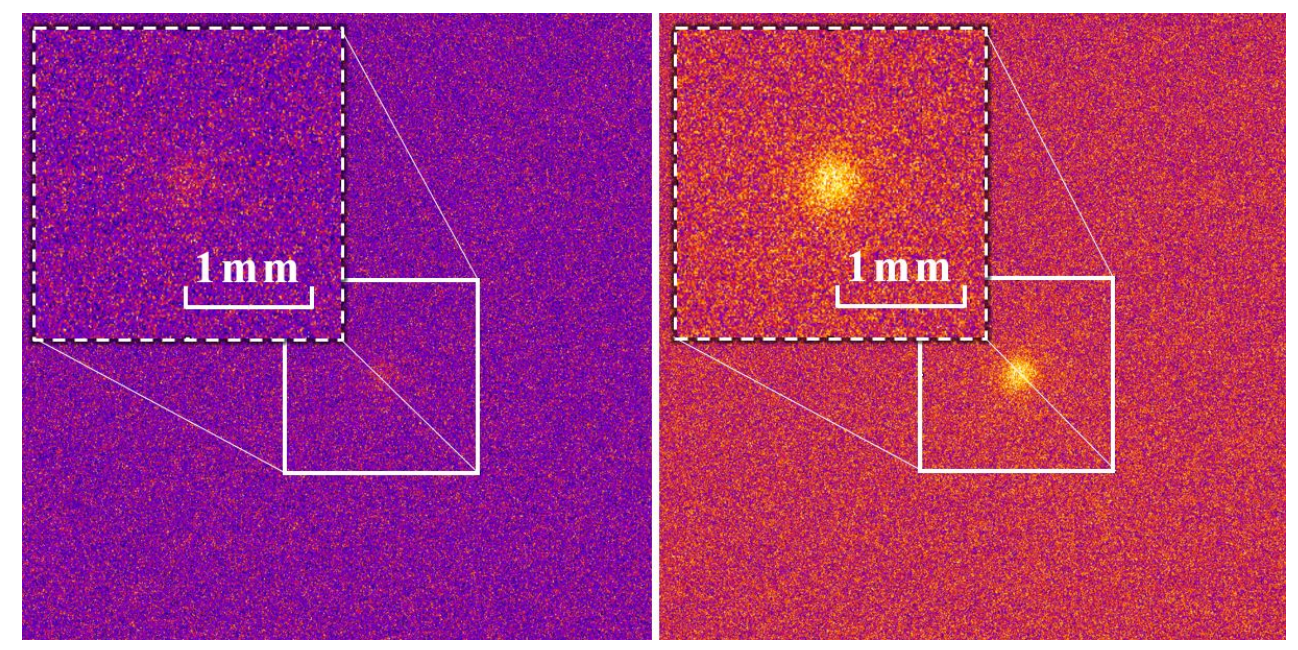

FIGURE IV.24: X-ray images formed by the spherical Bragg crystal for proton acceleration experiment 2. X-ray spot from a signle shot (left). X-ray spot summing 10 shots (right).
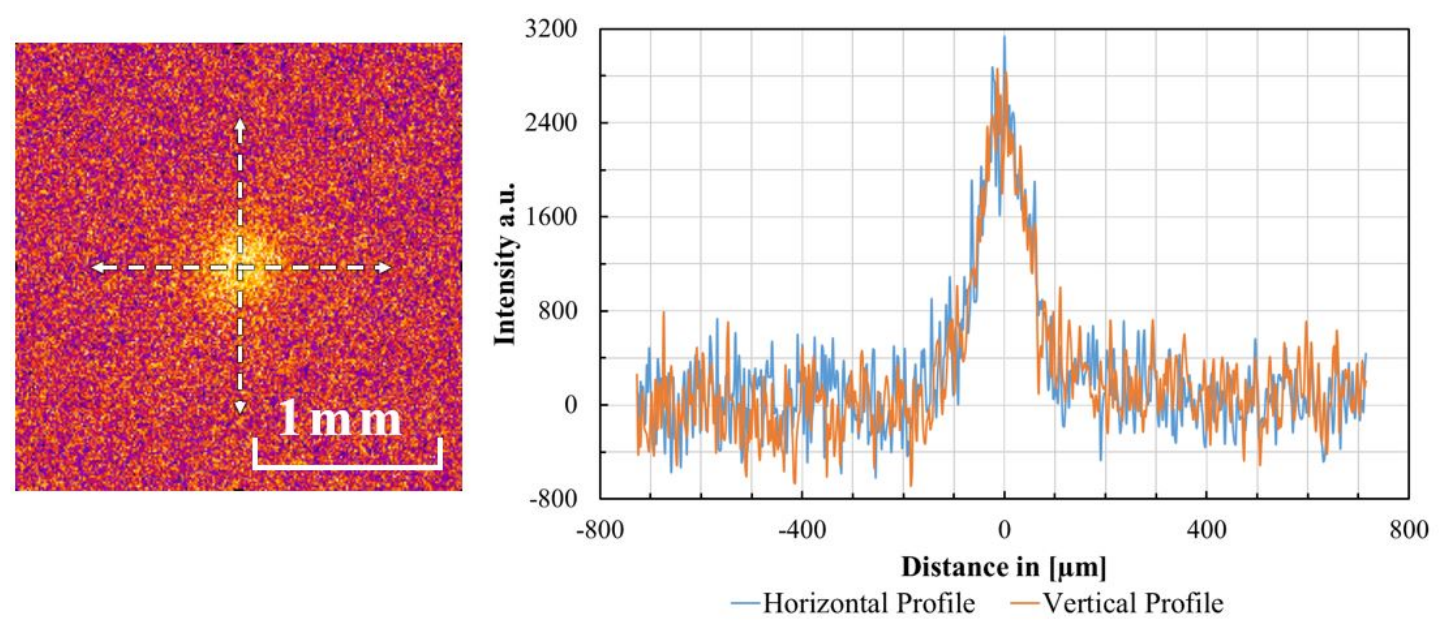

FIGURE IV.25: X-ray spot obtained after summing 10 laser shots (left). Corresponding real size after removing the magnification factor (right).

According to the data shown in Fig.IV.27, we can see a clear correlation between the K $\alpha$ signal on the rear side and the target thickness. The thicker the target is, lower is the K $\alpha$ signal. This decrease of signal is mainly due to the electron collisions in matter. On the other hand, the high intensity of the signal seen in the thinner $\mathrm{Al}$ target is enhanced due to the refluxing effect. The results obtained with the KB microscope can be even one order of magnitude higher in terms of signal to noise ratio if compared with Bragg crystal results. The experimental results also show that with even a larger solid angle, a few tens of milliradians ( $\sim 50 \mathrm{mrad})$ for the Bragg crystal compared to few of milli-radians $(\sim 5 \mathrm{mrad})$ for the $\mathrm{KB}$, the reflectivity is at least two orders of magnitude lower for the Bragg. Another disadvantage of using the Bragg crystal, relative only to this case, is the limitation in terms of number of shots due to targetry. Indeed, working at normal incidence angles implies having an adapted holder with free spaces to free the path between the Bragg crystal and the X-ray CCD camera (see Fig.IV.21) different from one shown in Fig.IV.15. 


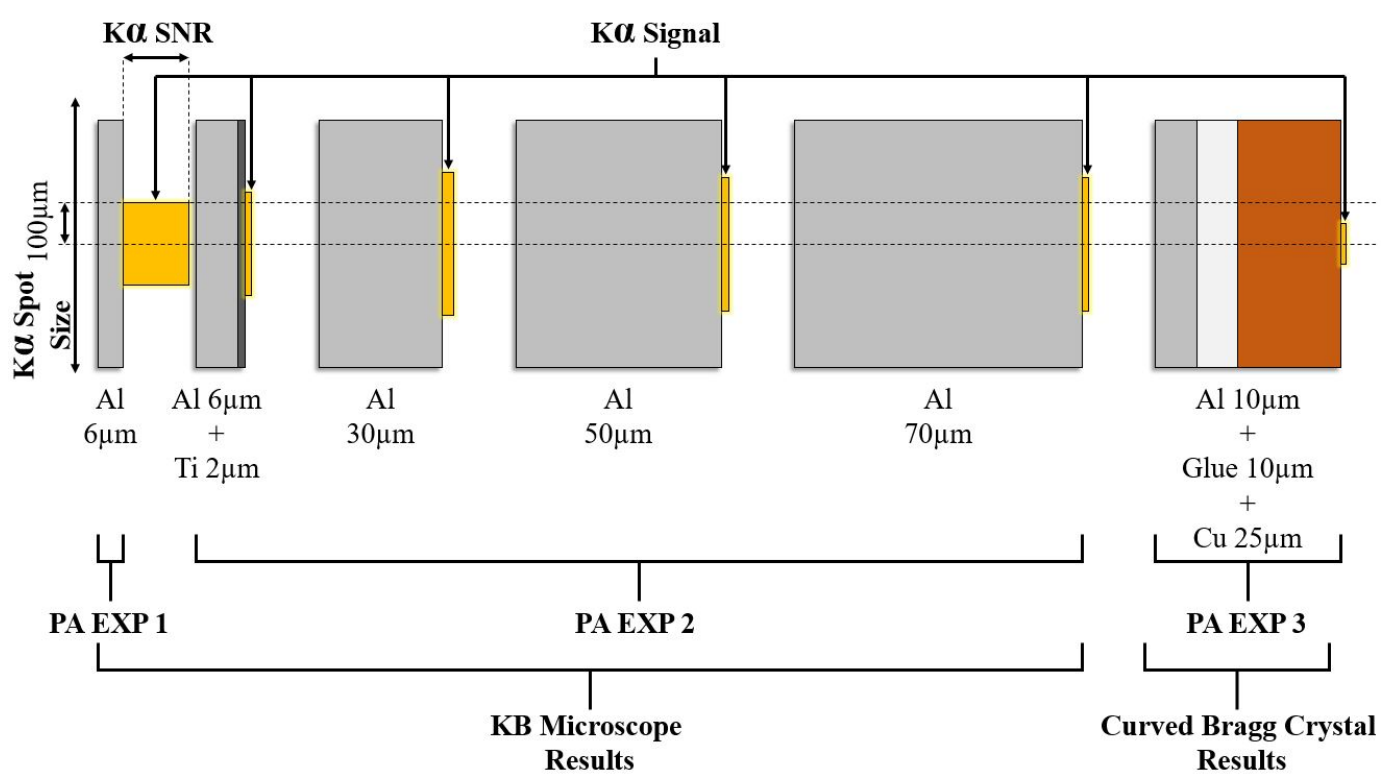

FIGURE IV.26: Full view results summary for all targets used in proton acceleration experiments 1,2 and strong shocks generation experiment 1 together with the $\mathrm{K} \alpha$ signal in terms of size and intensity.

\begin{tabular}{|c|c|c|c|c|c|c|}
\hline X-Ray Optic & Experiment & Target & $\mathbf{K} \boldsymbol{\alpha}-\operatorname{Size}[\mu \mathrm{m}]$ & Signal & Background & SNR \\
\hline \multirow{5}{*}{$\begin{array}{c}\text { KB } \\
\text { Microscope } \\
\text { (Al-Ka) }\end{array}$} & PA 1 & $\mathrm{Al} 6 \mu \mathrm{m}$ & 200 & 16667 & 1022 & 16,31 \\
\hline & \multirow{4}{*}{ PA 2} & $\mathrm{Al} 10 \mu \mathrm{m}+\mathrm{Ti} 2 \mu \mathrm{m}$ & 270 & 1488 & 922 & 1,61 \\
\hline & & Al $30 \mu \mathrm{m}$ & 355 & 2400 & 875 & 2,74 \\
\hline & & $\mathrm{Al} 50 \mu \mathrm{m}$ & 325 & 1675 & 888 & 1,88 \\
\hline & & $\mathrm{Al} 70 \mu \mathrm{m}$ & 330 & 1389 & 898 & 1,55 \\
\hline $\begin{array}{c}\text { Curved } \\
\text { Bragg Crystal } \\
(\mathbf{C u}-\mathbf{K} \alpha) \\
\end{array}$ & PA 3 & $\begin{array}{l}\mathrm{Al} 10 \mu \mathrm{m}+\mathrm{Cu} 25 \mu \mathrm{m} \\
+ \text { Glue } 10 \mu \mathrm{m}\end{array}$ & 100 & 1064 & 809 & 1,31 \\
\hline
\end{tabular}

FIGURE IV.27: Quantitative data summary to compare K $\alpha$ spot size, signal and SNR for different $\mathrm{X}$-ray diagnostics and experiments.

\section{IV.3.2 The KB microscope for focused secondary $x$-ray sources}

In this section we are introducing another application of the $\mathrm{KB}$ microscope which is beam transport. Up to now, we have presented in detail the usual application of the KB microscope, which consists mainly of imaging $\mathrm{X}$-rays. Obviously, operating as an $\mathrm{X}$-ray imager, it is basically transporting $\mathrm{x}$-rays. In fact, having a diagnostic with this ability permits the creation of dense secondary $x$-ray sources free of charged particles. This property can be very useful for many applications in laser-plasma experiments such as the x-ray probing of matter for spectroscopy applications [Mo et al., 2013]. Another alternative for using these secondary x-ray sources is to perform x-ray radiography in low noise environments i.e the closer are made the measurements to TCC (Target Chamber Center) and the noisier the acquisition will be. Fig.IV.28 shows an example of a possible configuration for using the KB microscope in laser-plasma experiments. In the proposed scheme, the primary laser driven $\mathrm{x}$-ray source is generated via LWFA in the form 
of betatron radiation (using the same configuration, the gas target can be also replaced by a solid one for bremsstrahlung generation). In addition, using an adjustable KB microscope allows to have more flexibility in experimental layouts. With the property of focusing $\mathrm{x}$-rays in different focal planes, the adjustable KB microscope permits the full control of the x-ray intensity on target i.e. the shorter is the $\mathrm{KB}$ focusing distance and the higher is the density of the $\mathrm{x}$-rays (and vice versa). Fixing the distance of the $\mathrm{KB}$ to the sample and defocusing the $\mathrm{KB}$ one can reduce the $\mathrm{x}$-ray intensity on the sample.

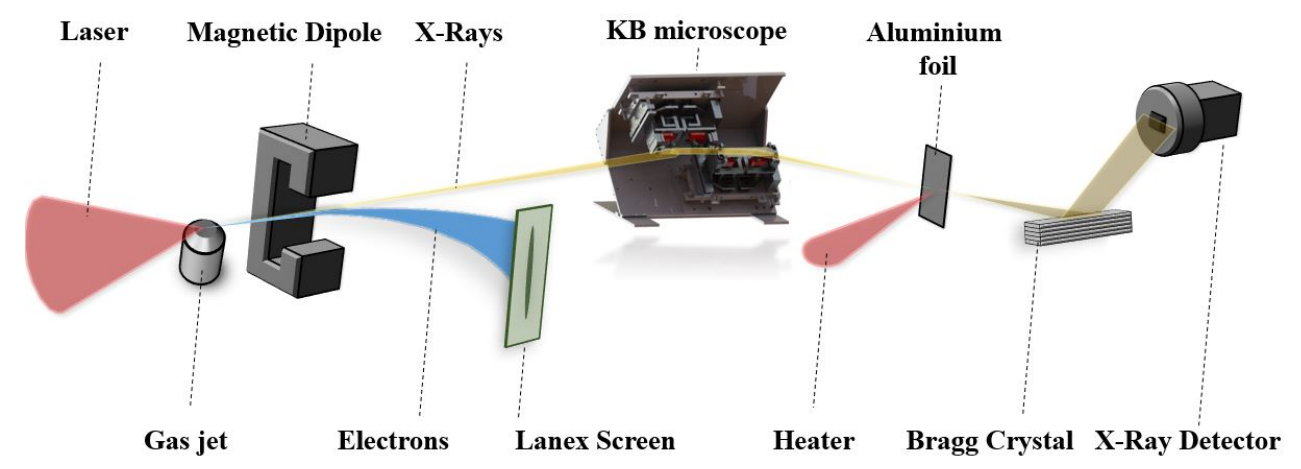

FIGURE IV.28: Use of the adjustable $\mathrm{KB}$ microscope for $\mathrm{x}$-ray transport in a previous experimental campaign in CLPU.

To conclude this section, we report that an experiment similar to the one of [Mo et al., 2013] has been carried out in CLPU where the goal of the activity was to probe the ionisation states of aluminium in a Warm Dense state using betatron radiation. The latter was first generated via LWFA and then transported via the adjustable KB microscope (the same model used in proton acceleration experiments 1 and strong shocks generation experiment 1). The high energy electrons generated in LWFA interaction were deviated using a magnetic dipole of $1.2 \mathrm{~T}$ in a similar experimental configuration as shown in Fig.IV.28. Separated from the charged particles, the emitted betatron radiation was then transported and focused using the KB in a laser-preheated aluminium thin foil $(\sim 500 \mathrm{~nm})$ using a $1 \%$ fraction of VEGA 2 at high power $\left(\lambda_{L}=800 \mathrm{~nm}, 30 \mathrm{fs}\right.$ pulse duration, $0.06 \mathrm{~J})$. More details about electron and betatron characterisation are presented in the next section. The application mentioned in this paragraph is only one of many more where the KB microscope can play a key role in $\mathrm{x}$-ray transport.

\section{IV.4 X-ray based applications: X-ray Phase Contrast Imaging XPCI}

\section{IV.4.1 Principles for XPCI}

$\mathrm{XPCI}$ is a very promising imaging technique based on $\mathrm{x}$-ray phase shifting of photons due to the refractive index of materials and diffraction enhancement of local density gradients in the material. XPCI is an alternative to absorption based $\mathrm{x}$-ray radiography. During transport, $\mathrm{x}$ ray photons are deflected from higher to lower density regions. An interference pattern is then created at the detector's plane where the local density gradient are more visible with fringes of 
high intensity. Fig.IV.29 shows the principle for propagation based x-ray phase contrast imaging.

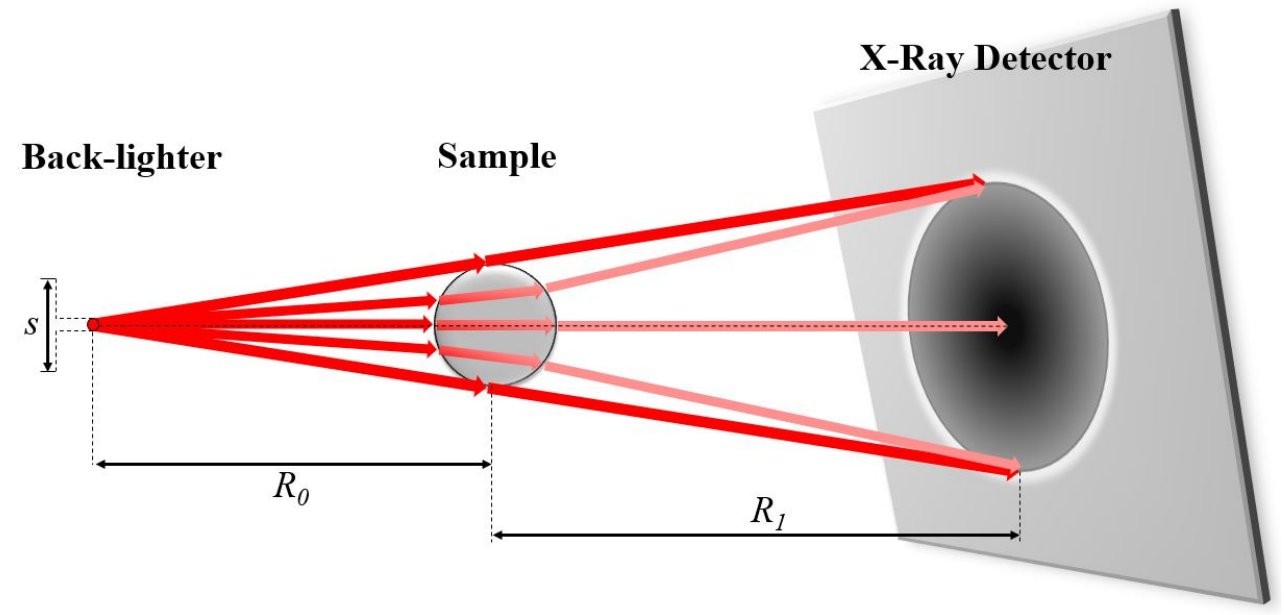

FIGURE IV.29: Point projection radiography setup for XPCI scheme.

Remembering that the complex index of refraction, $\mathrm{n}^{*}$, can be written as $\mathrm{n}^{*}=1-\delta$-i $\beta$ (Eq.II.36), we can say the phase shifting factor $\delta$ is responsible for the high brightness zones on the detector, meanwhile the absorption factor $\beta$ is responsible for the transmission variations.

According to [Diemoz et al., 2012], the intensity at the $\mathrm{x}$-ray detector $\mathrm{I}_{\text {detector }}$ is given by

$$
\mathrm{I}_{\text {detector }}=\frac{\mathrm{I}_{0} \mathrm{~T}}{M^{2}}\left(1-\frac{\lambda R_{1}}{2 \pi M} \nabla_{\perp}^{2} \phi\right)
$$

Where $\mathrm{I}_{0}$ is the intensity incident onto the object, $M=\left(R_{0}+R_{1}\right) / R_{0}$ is the magnification factor, $\mathrm{T}=\exp \left(-\int d z \beta\right)$ is the object transmission (where $\beta$ is the linear attenuation coefficient) and $\phi$ is the phase shift produced by the object defined by [Wu and Liu, 2003] as

$$
\phi=\frac{2 \pi}{\lambda} \int \delta(z) d z
$$

Ideally, free electron lasers and synchrotrons are the most suitable facilities to carry out XPCI experiments since they can deliver high flux of highly coherent (temporally and spatially) $\mathrm{x}$-ray beams. On the other side, laser driven $\mathrm{x}$-ray sources is also a very interesting alternative to carry out XPCI. It is true that these x-ray sources are broadband and incoherent but, their small size can still be small enough to permit XPCI applications. A possible scheme for laser-driven x-ray generation would be via LWFA in form of broadband betatron radiation [Fourmaux et al., 2011]. Another possibility to generate laser driven X-rays, while allowing for XPCI, would be via lasersolid interaction in the form of bremsstrahlung emission. The only condition to satisfy here again would be to have a source relatively small (of few microns). In other words, the transverse coherence length $l_{c}$ should be larger than the scale length for the structure to be resolved [Kneip et al., 2011]. According to [Antonelli et al., 2019] $l_{c}$ is defined as

$$
l_{c} \simeq \frac{R_{0} \lambda}{s}
$$


where $R_{0}$ is the distance from the X-ray source to the sample, $s$ is the source size and $\lambda$ is the $\mathrm{x}$-ray wavelength.

\section{IV.4.2 XPCI experiment 1 (R. Fedosejevs 2017)}

\section{Experimental setup}

We are presenting in this section the results of an experimental campaign whose aim is to carry out XPCI for biological samples using betatron radiation. VEGA 2 laser, at full power (6J, 30fs), was focused by a long focal off-axis parabola (OAP) $(\mathrm{f}=130 \mathrm{~cm}$, where $\mathrm{f}$ is the focal length of the parabola) into a gas jet of pure helium with an intensity on target of $\sim 4 \times 10^{19} \mathrm{~W}_{\mathrm{cm}} \mathrm{cm}^{-2}$ to accelerate electrons up to relativistic energies via LWFA. Optimizing the conditions needed for self injection was carried out by varying gas pressure (density modification) and focal position (density and $a_{0}$ ) with respect to the gas nozzle. Due to its small size in the transverse dimension (of the order of the size of the bubble $\sim 30$ microns) and low divergence ( $\sim$ few mrad, with a high density of photons in the emission cone), the generated betatron radiation was used to carry out XPCI for two biological samples (a plant leaf and a fly's wing) placed together in the same sample holder. My contribution consisted of the mounting of the experimental setup and the carrying out of both acquisition and data analysis. Fig.IV.30 shows the experimental setup used for the XPCI experiment 1 where the magnification $M$ of the system is 1.98 . The samples used for XPCI, shown in Fig.IV.31, were chosen for both, their high transmission in the X-ray range and also for their small micro-structures (later used for resolution measurements).

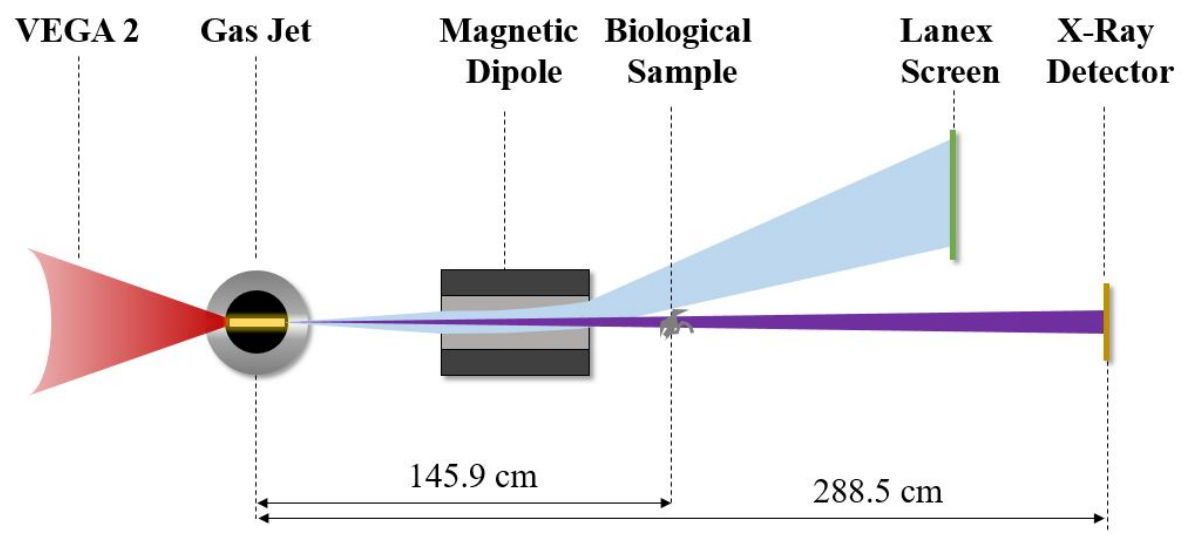

FIGURE IV.30: Experimental setup for XPCI experiment 1.

\section{Results: XPCI for biological samples}

The flux of generated x-ray photons per shot was enough to construct images of reasonable contrast. Fig.IV.32 shows some examples of the raw data obtained for different shots showing that, while noisy, single shot images are possible.

In addition to the relatively low signal to noise ratio per shots (SNR 6 ), pointing variations (in the order of $\sim 27 \mu \mathrm{rad}$ ) made the analysis more complex. After aligning the images and adding a total of 9 shots we have been able to reconstruct the image seen in Fig.IV.33 


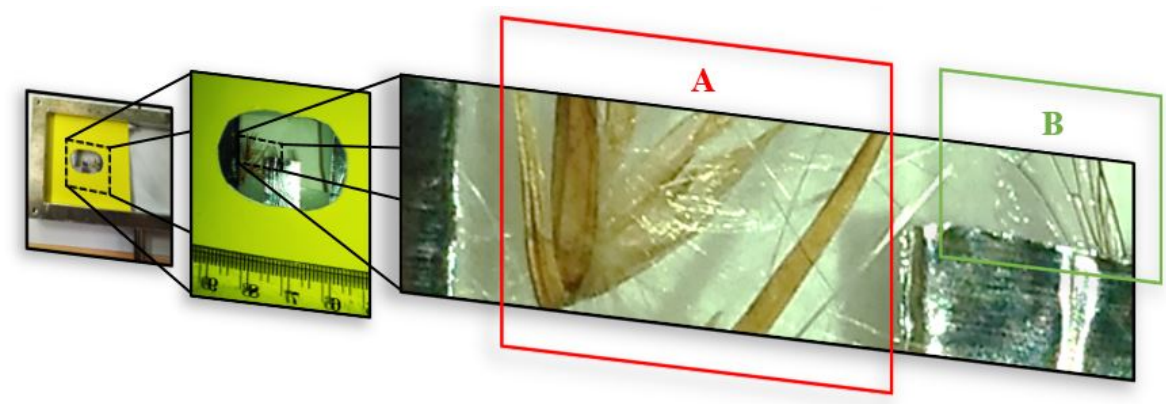

FIGURE IV.31: Sample used for XPCI experiment 1. Zone A contains the leaf meanwhile zone B contains the wing of a fly.
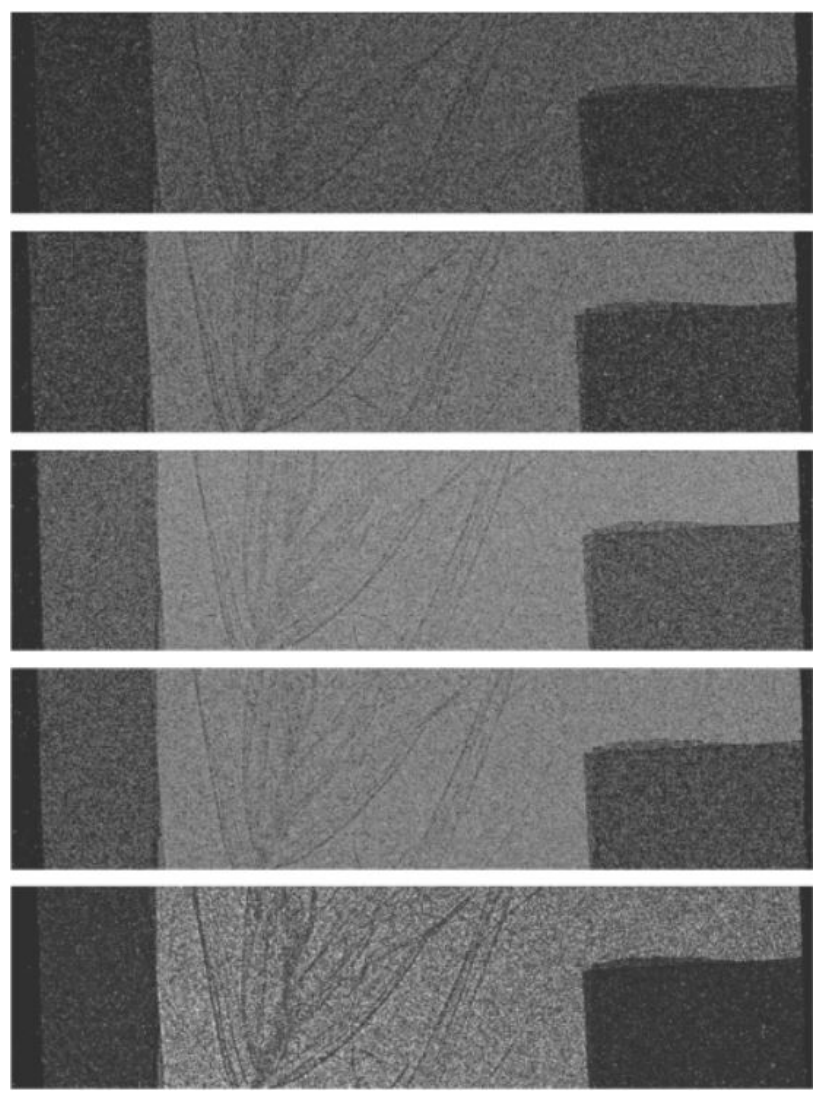

FIGURE IV.32: Examples of raw data obtained for XPCI experiment 1. The pictures are organised in function of the intensity of the x-ray signal (from lower in the top to higher in the bottom).

The reconstructed image of Fig.IV.33 has a maximum resolution of $13 \mu \mathrm{m}$ which is basically the size of the pixel divided by the magnification factor M. Fig.IV.34 is showing the profile of the smallest structure resolved with this system, in which, we can notice the role of the phase shift enhancement of the signal at the edges of the leaf (dotted green circle). One can also appreciate the decreasing of the signal due to the absorption factor $\beta$ (dotted orange circle).

Fig.IV.35 shows another example of phase contrast in which the interferences at the edges of the irradiated fibre can be observed on both edges. 


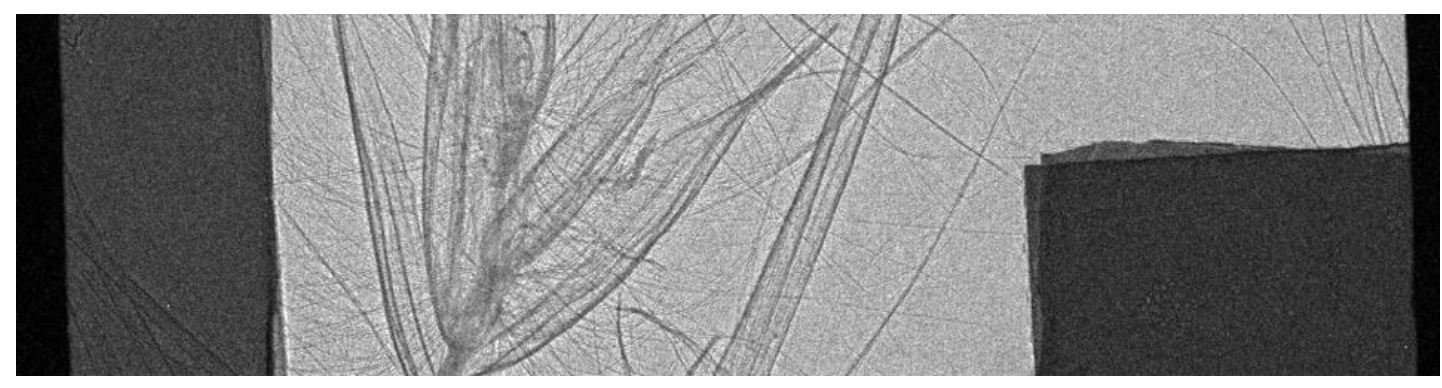

FIGURE IV.33: Reconstructed image (leaf in the center and a fly wing in the right corner) from XPCI experiment 1 . The size of the picture is equal to the size of the Greateyes chip which is equal to $26624 \times 6656 \mu \mathrm{m}$ with $26 \mu \mathrm{m}$ pixel size.
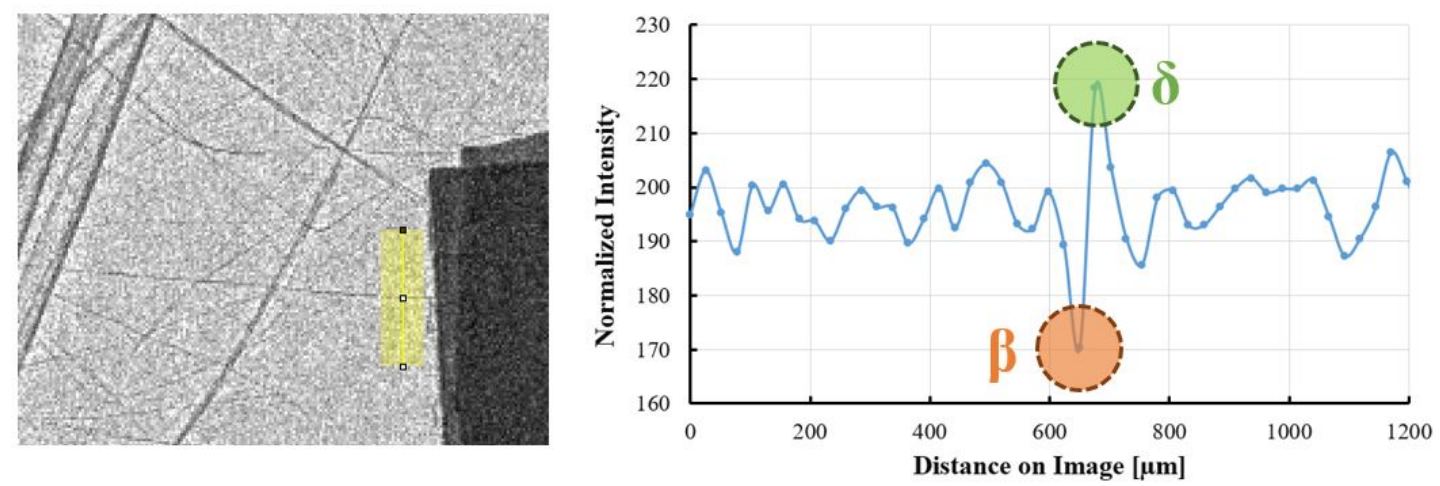

FIGURE IV.34: Profile of the smallest structure of the organic sample (Right). The region from which the profile was taken (left).
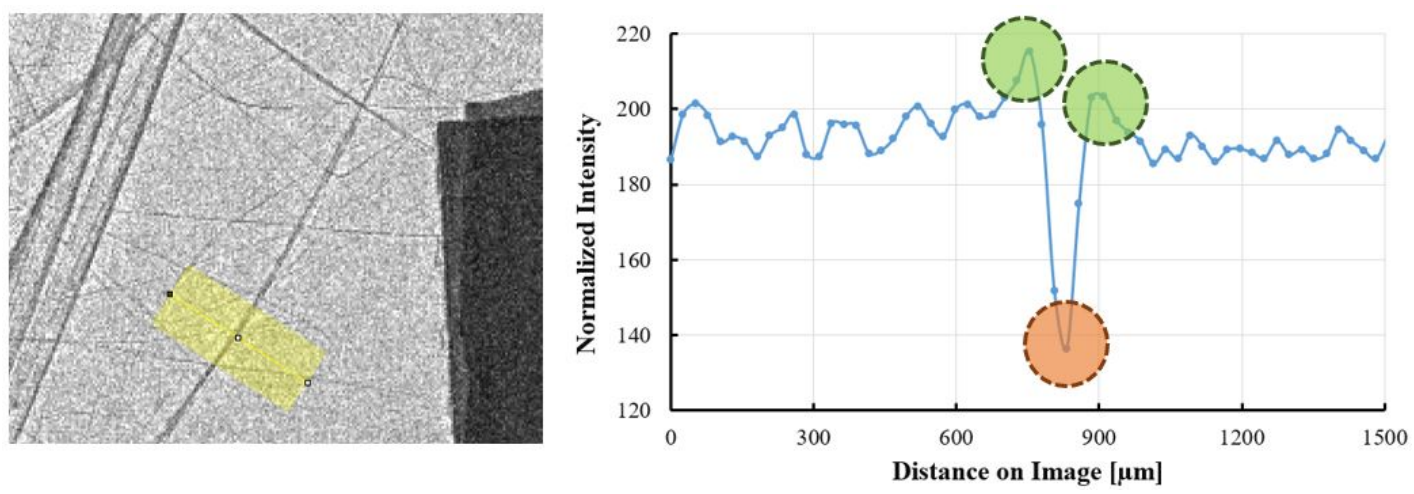

FIGURE IV.35: Profile of a given structure with an important phase shift at both of its extremes (right). Reference region from which the profile was drawn (left).

The results obtained in this section have shown the importance of using XPCI for x-ray imaging of high transmission low contrast samples. We have also proven that XPCI can be carried out even with a temporally incoherent $\mathrm{x}$-ray source of small spacial extent such as LWFA-driven betatron (if the condition of being relatively small when compared with the features of the sample to be radiated is satisfied). The results shown here are only a preliminary example and a more detailed study must be carried out for a better characterisation of the x-ray source in terms of brilliance, spatial and spectral profile. A higher brilliance may be achieved by controlling the 
electron injection into the accelerating structure. More are the electrons injected and more are the x-rays produced, and more brighter the results can become. Using high repetition rate ultrashort ultra-intense lasers to drive betatron in under-dense matter is a very promising technique which increases the data acquisition rate and opens a new door for many other applications.

\section{IV.4.3 XPCI experiment 2 (L. Antonelli 2017)}

We present in this section the results of an experimental campaign carried out at PHELIX facility [Neumayer et al., 2005] where we have used XPCI to study a laser induced shock wave. A 50J laser pulse was equally divided in two parts. One short pulse of 500fs duration to generate X-rays and another long one of $2 \mathrm{~ns}$ to generate a shock wave. The first part of the campaign consisted of carrying out successful XPCI radiography of static objects by modifying the distances between the $\mathrm{x}$-ray back-lighter and the target, and also the one from the target to the x-ray detector. The goal of the second part of the campaign was to apply XPCI to a travelling shock wave. My contribution here consisted of the building of the experimental setup of the experiment, to data analysis and to post-experiment discussions. From this experiment, three articles have been successfully published in peer reviewed journals [Antonelli et al., 2019, Barbato et al., 2019a, Barbato et al., 2019b].

\section{XPCI for static objects}

We have used at the beginning only the first half of the beam, the short pulse one with $25 \mathrm{~J}$, $500 \mathrm{fs}$ and $1.06 \mu \mathrm{m}$, focused onto a $5 \mu \mathrm{m}$ diameter tungsten wire with a $5 \mu \mathrm{m}$ focal spot leading to intensity on target of $\sim 6 \times 10^{19} \mathrm{Wcm}^{-2}$. According to [Schönlein et al., 2016], $10 \%$ to $20 \%$ of laser energy is converted into relativistic electrons. These ones are transported into the target and emit Bremsstrahlung radiation. The latter is then used as an X-ray back-lighter to to perform XPCI. Fig.IV.36 summarises the experimental setup used for XPCI optimisation.

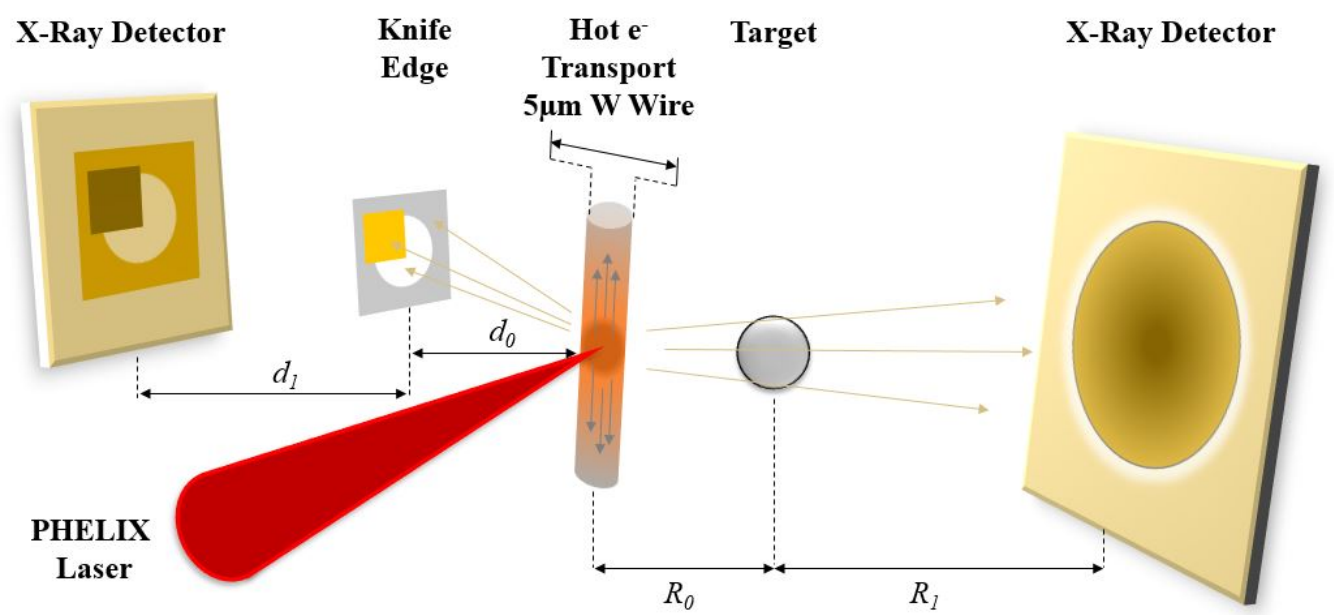

FIGURE IV.36: Experimental setup for XPCI experiment 2 part 1.

The $\mathrm{x}$-ray source size was characterised using the knife edge technique using a thin gold foil $(5 \mu \mathrm{m})$ attached to an aluminium holder using thin Kapton tape. The latter is widely used (by the 
experimental laser-plasma community) because it is easy to manipulate, is vacuum compatible and is highly transmissive for $\mathrm{x}$-rays (see Fig.IV.37). Knife edge technique consists of deducing the real size of the emitting $x$-ray source by carrying out x-ray radiography of a sharp object (usually few microns thick). The sharper is the shadow, the smaller is the source size.

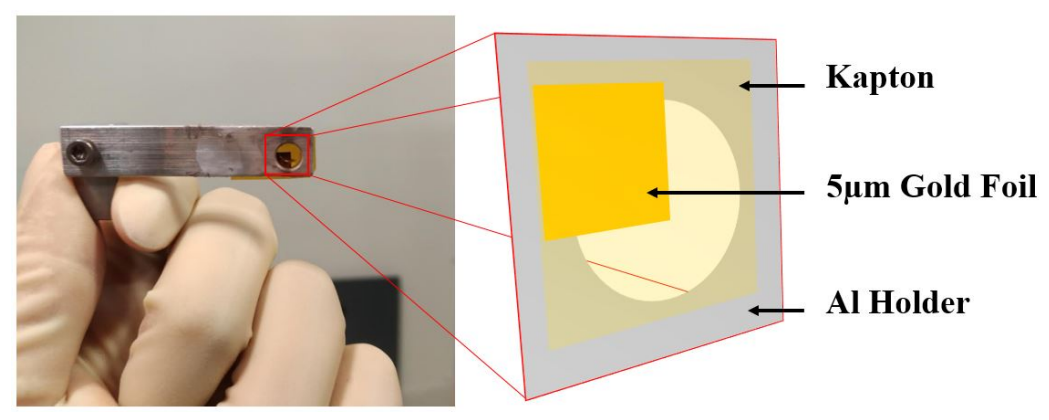

FIGURE IV.37: Gold foil holder for knife edge measurements.

The knife edge target was placed at $d_{0}=5.5 \mathrm{~cm}$ from the x-ray source. An IP was used as $\mathrm{x}$-ray detector for the radiography and was placed at $d_{1}=152 \mathrm{~cm}$ away from the knife edge target holder. The magnification $M_{\mathrm{KER}}$ of this imaging radiography (KER stands for Knife Edge Radiography) is given by Eq.IV.11 and is equal to 28.6.

$$
M_{\mathrm{KER}}=\frac{d_{0}+d_{1}}{d_{0}}
$$

Radiography measurements results using IP and corresponding profile plots in the horizontal and vertical dimensions are shown in Fig.IV.38.

From the obtained profiles we could deduce the size of the x-ray source in both horizontal and vertical axis using the following equation

$$
\Delta_{x, y}=\frac{\operatorname{Pix} \times N_{x, y}}{M_{\mathrm{KER}}}
$$

where $\Delta_{x, y}$ is the real dimension of the x-ray source in the horizontal and vertical axis, $x$ and $y$ respectively. Pix is the pixel size (user defined by the scanner) and $N$ is the number of pixels from $10 \%$ to $90 \%$ intensity, defined in Fig.IV.38. After calculation, the resulting source size in the horizontal axis was $\Delta_{x} \sim 5 \mu \mathrm{m}$ which is basically the initial thickness of the wire and in the vertical one was $\Delta_{y} \sim 30 \mu \mathrm{m}$. In addition to this and knowing that the emitted x-rays are in the range of, $1 \AA$ to $2 \AA$, we can estimate the coherence length of the system $l_{c}$. The latter is maximum the horizontal plane (where the size of the source is smaller) and is equal to $10 \mu \mathrm{m}$ (for $R_{0} \sim 25 \mathrm{~cm}$ ) meanwhile it decreases in the vertical dimension down to $1 \mu \mathrm{m}$. The data shown later are confirming the success of the XPCI under these conditions.

We have used fishing wires made basically from nylon, shown in Fig.IV.39, as targets for XPCI optimisation while varying the magnification of the system. Fig.IV.40 summarises the results obtained with four different conditions A, B, C and D. The XPCI results shown in A,B and C were obtained using an Andor IKON-M x-ray camera of $13 \mu \mathrm{m}$ pixel size meanwhile the results shown in D are obtained using an IP. 

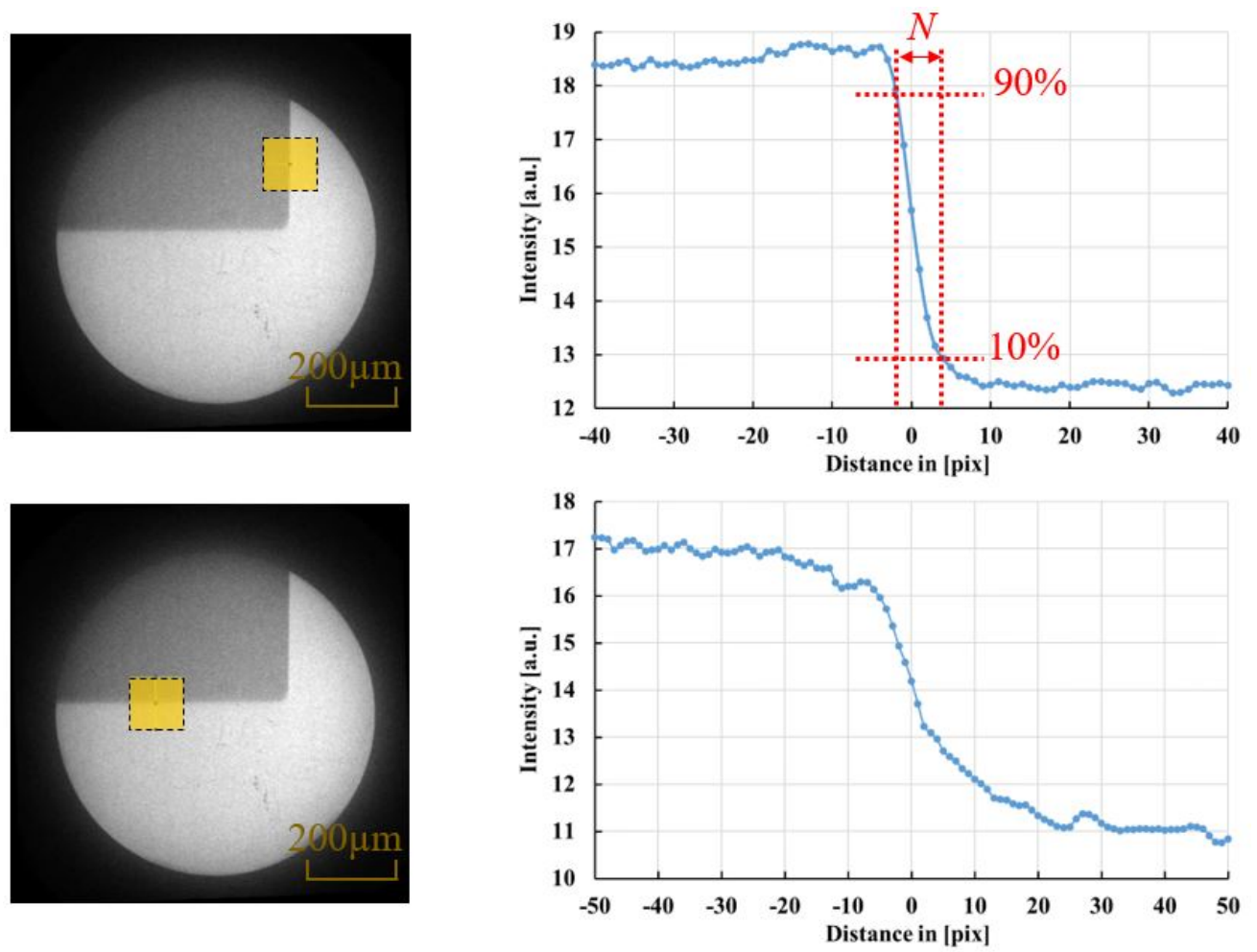

FIGURE IV.38: Real measurements on IP of the x-ray source size via knife edge technique (left). Profile of the source in the horizontal and in the vertical axis respectively on the right top and right bottom of the picture.

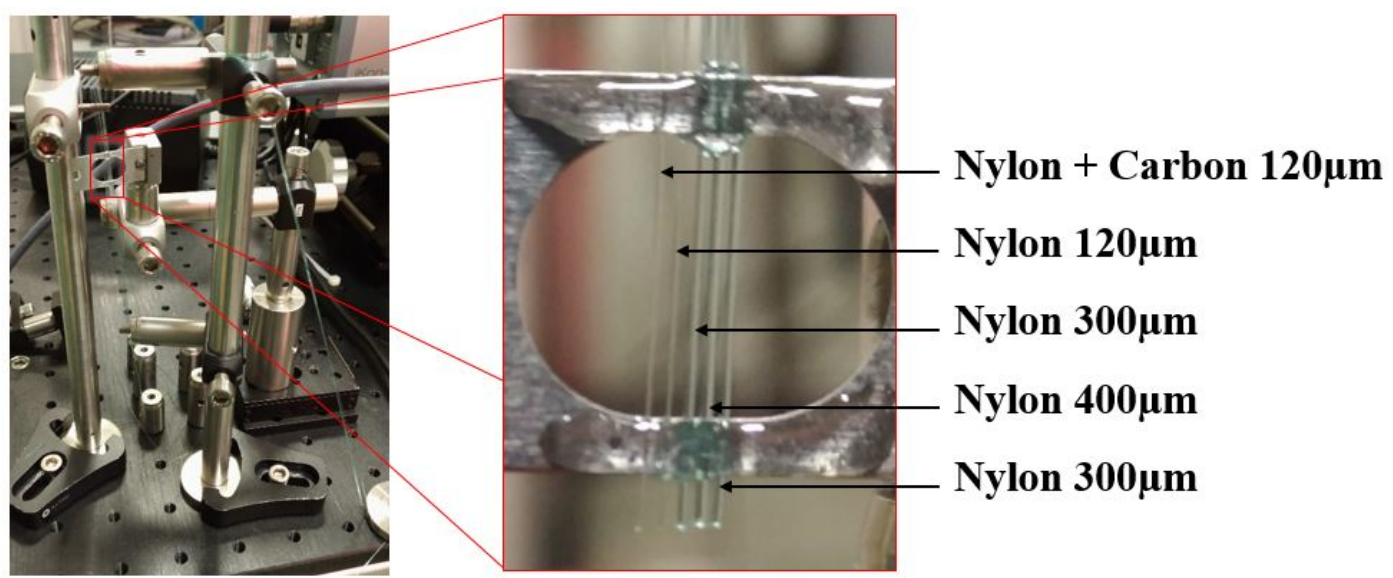

FIGURE IV.39: Different targets used for XPCI signal optimisation.

From the radiography profiles shown in Fig.IV.40.left, we can see that the results obtained on the IP are smoother if compared to the ones with the X-ray CCD which seem to be blurry. This is due to the difference between the two detectors in term of both, dynamic range and quantum efficiency. For both detectors we could clearly observe the phase shift enhancement on the edges of the samples at the interface between the wires and vacuum where the signal level increases by up to $10 \%$ for some cases. Also, the contribution of the absorption coefficient $\beta$ is clearly 

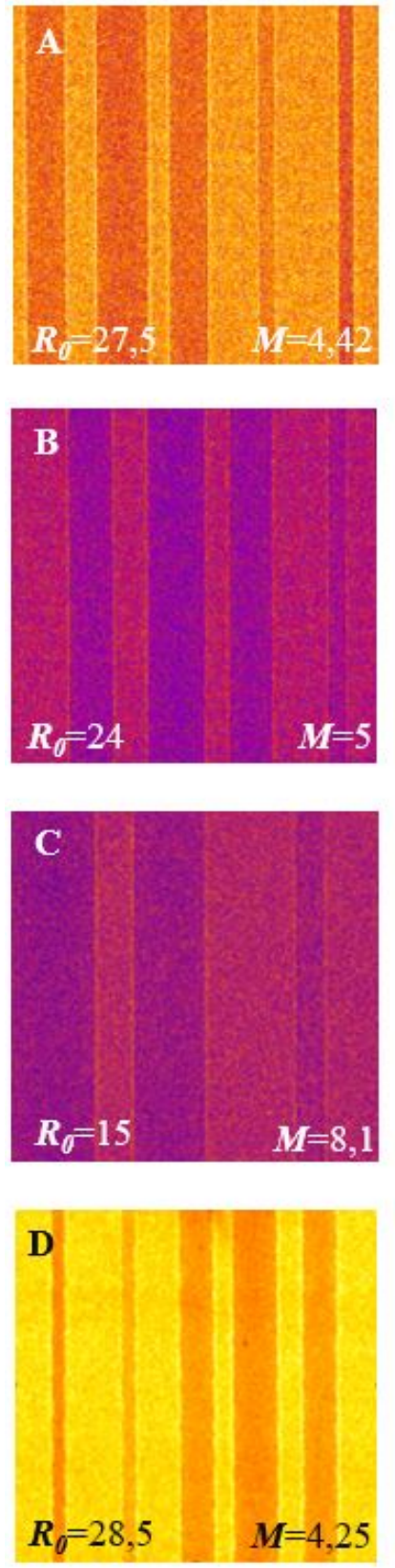
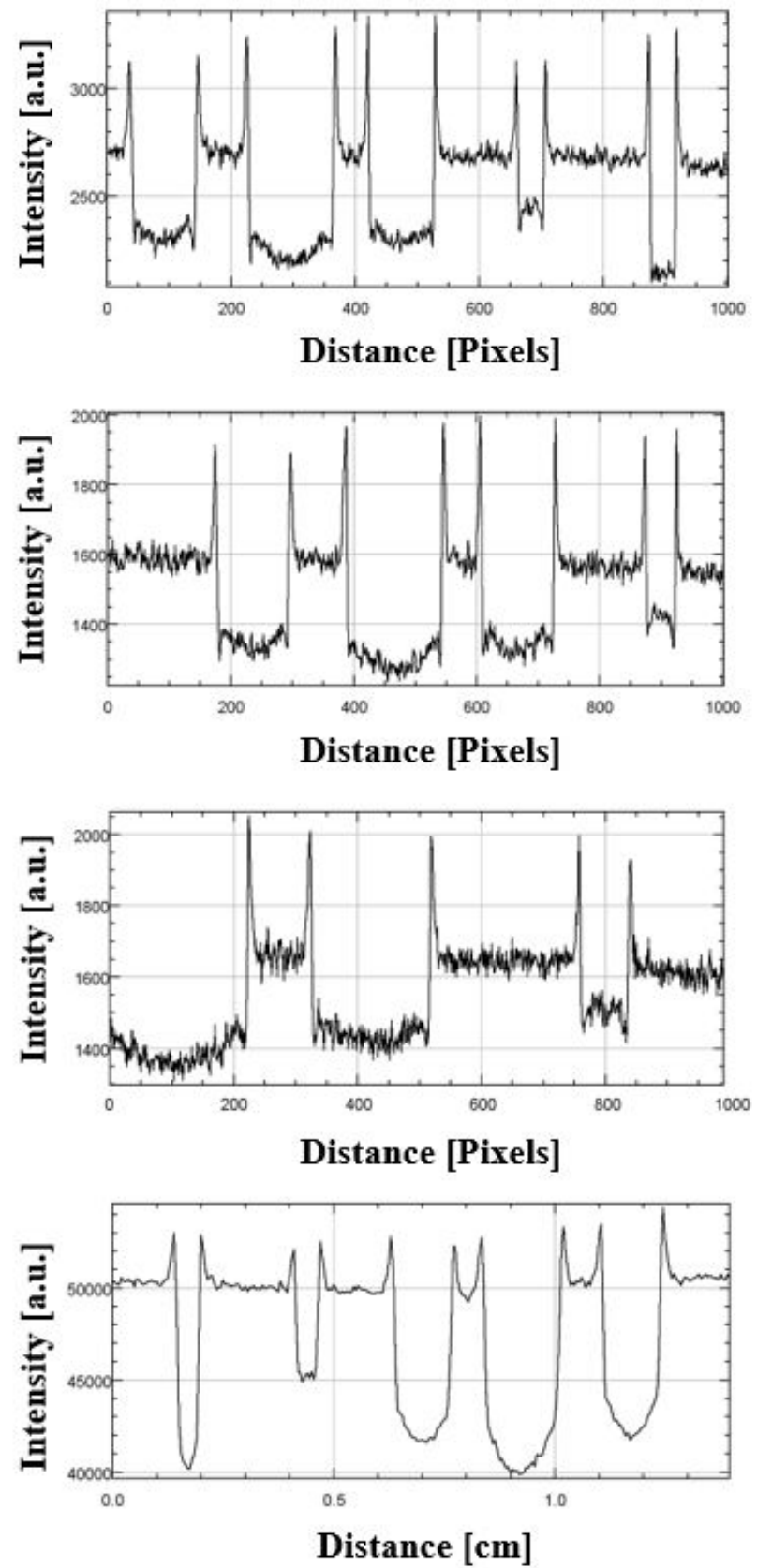

FIGURE IV.40: XPCI of the same nylon wires for different magnification configurations A,B and C using an X-ray CCD camera and D using IP (left). Corresponding profile plots (right).

observed where the thicker the wire, the higher is $\beta$ and the lower is the transmitted signal. We also note a decrease of transmission for targets of the same thickness but with higher density (nylon+Carbon wire versus the pure nylon wire).

A cylindrical HOPG spectrometer (of interplanar distance $\mathrm{d}=0.315 \mathrm{~nm}$ ) was also installed in the experiment to monitor the high energy electrons. Fig.IV.41 (top) is showing the position of the HOPG respectively to the $\mathrm{x}$-ray source. The HOPG was adjusted to observe (on the IP) the 
emission lines $\mathrm{L} \alpha_{1}$ and $\mathrm{L} \alpha_{2}$ of tungsten corresponding respectively to the energies $8.39 \mathrm{keV}$ and $8.33 \mathrm{keV}$ in Fig.IV.41 (middle). The profile of the signal recorded on the IP is shown in Fig.IV.41 (bottom).

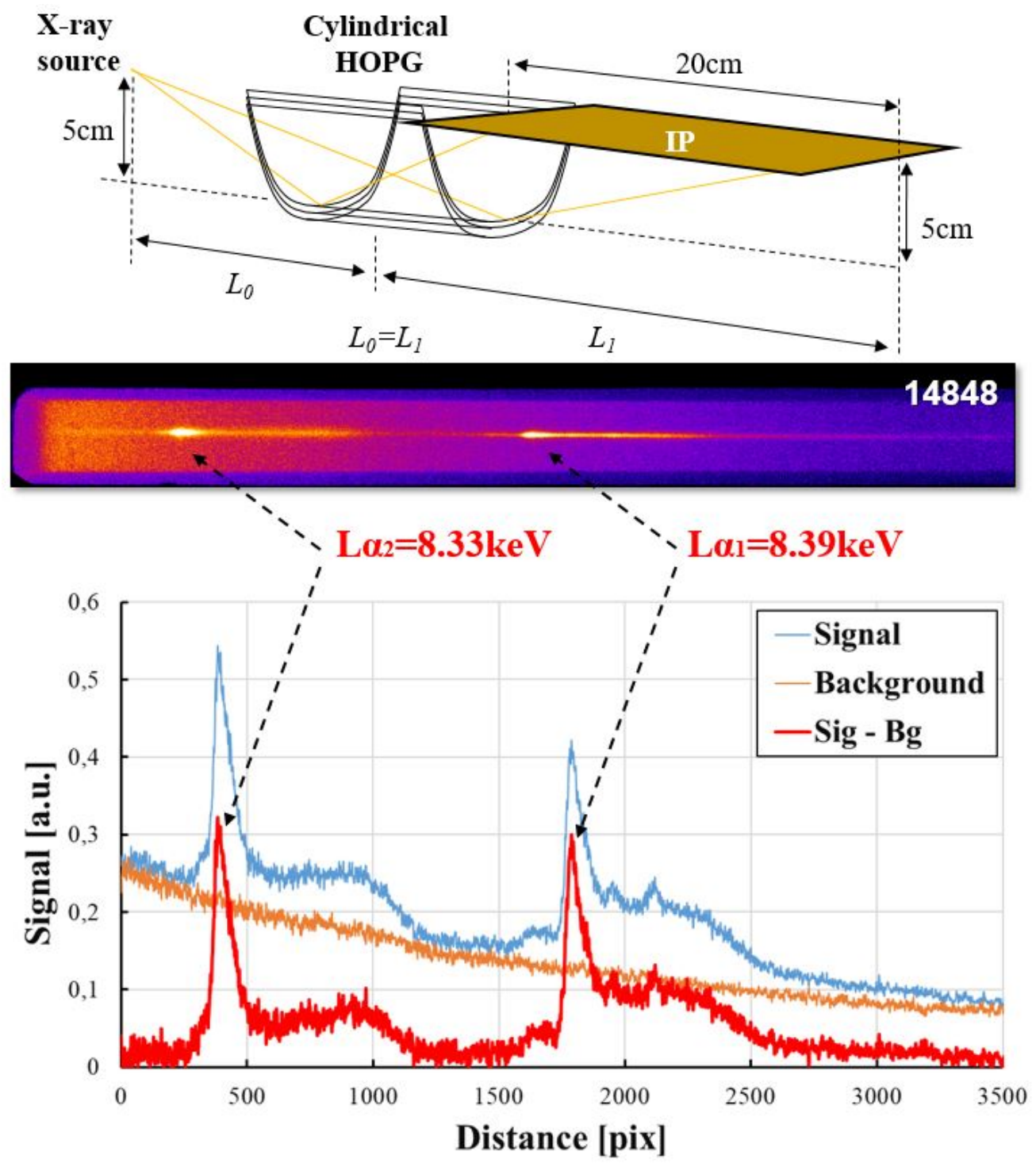

FIGURE IV.41: Experimental setup for HOPG installation (ToP). Traces on the IP from the $\mathrm{L} \alpha_{1}$ and $\mathrm{L} \alpha_{2}$ emission lines (Middle). Profile of the traces onto the IP (Botom).

\section{XPCI for dynamic targets}

In the previous section we have confirmed the success of XPCI at the conditions mentioned above (in XPCI experiment 1 part 1). The second part of the experiment consists of carrying out XPCI for a dynamic/moving target. A blast wave or a shock wave was driven using the second half of the uncompressed beam of PHELIX 25J, 2ns, $1.06 \mu \mathrm{m}$. The beam was focused to a $50 \mu \mathrm{m}$ spot to reach a intensity of $10^{14} \mathrm{Wcm}^{-2}$ on target. This target was made from plastic and had a 
cylindrical shape of $300 \mu \mathrm{m}$ diameter. Fig.IV.42 shows the final experimental setup used to carry out XPCI experiment 2 part 2.

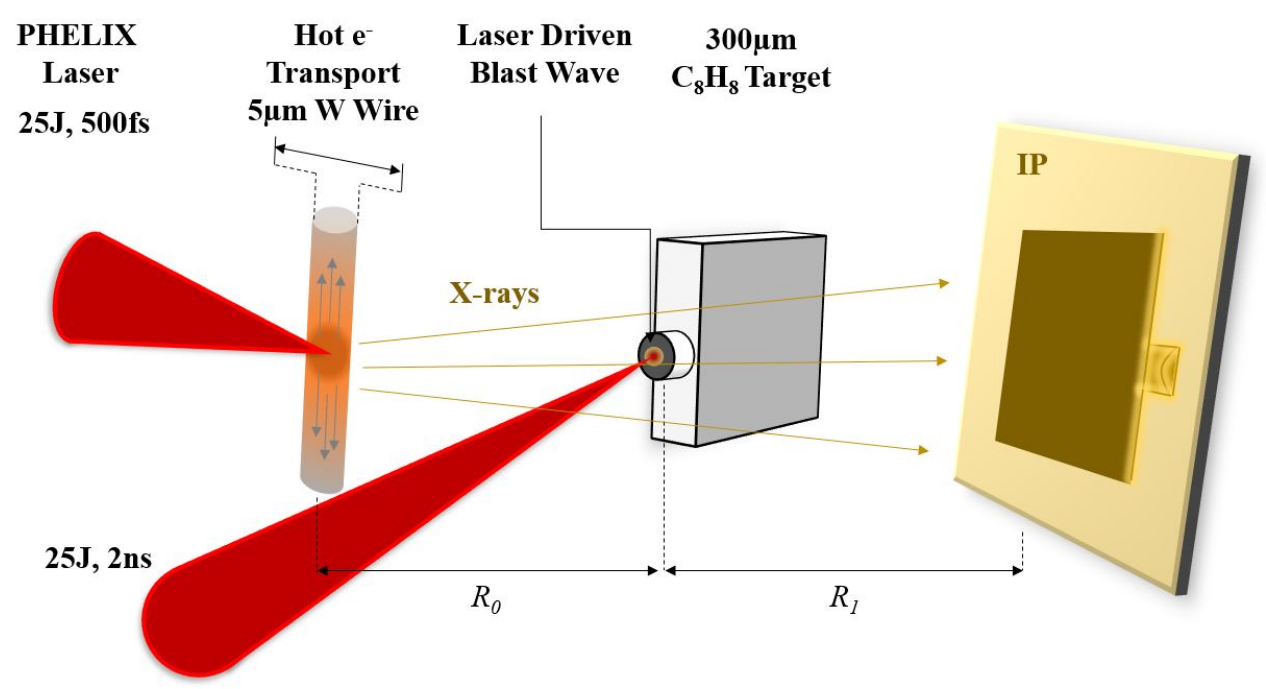

FIGURE IV.42: Experimental setup for XPCI experiment 2 part 2.

The knife edge and the HOPG were still installed but are not presented in the figure. The second half of the laser responsible of driving the shock is sent $6 \mathrm{~ns}$ before the first half, generating $\mathrm{x}$ rays, to give enough time to the blast wave to propagate. The data were acquired using imaging plates and the results of the XPCI radiography are shown in Fig.IV.43.

From the measured profile, in the horizontal direction, we can easily distinguish three important regions (in green colour) where the phase contrast is enhanced. The first region is the interface vacuum-target, while the second and the third ones are respectively the back and the front of the laser driven shock wave. None of these three regions would be visible if no XPCI radiography was used. X-ray absorption can also be seen in four regions. The first three are the ones where the target is cold and where the absorption is directly proportional to the thickness of the sample. The interesting part is the one in red colour, where the absorption is enhanced. The latter is due to the shock wave where high density of the compressed matter is travelling in the target itself.

We have proven that XPCI radiography can be successful even using incoherent laser-driven $\mathrm{x}$ ray sources such bremsstrahlung radiation. We have seen that using super thin targets such, $5 \mu \mathrm{m}$ tungsten wire, can permit the use of such x-ray sources for XPCI radiography purposes. The promising results obtained in this section open the possibility of implementing this technique to high energy density experiments making of XPCI an interesting tool to explore shock propagation in various systems including the target core used for ICF. 

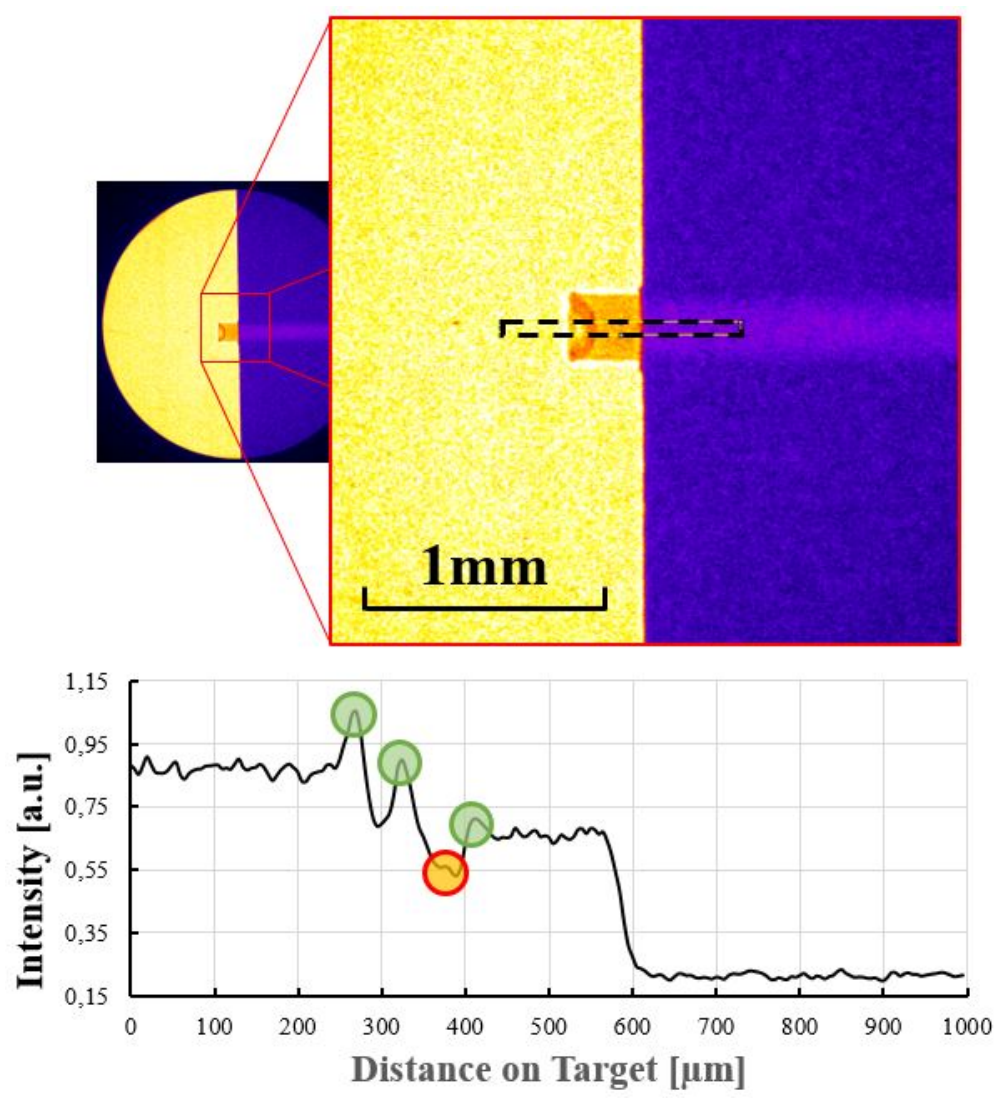

FIGURE IV.43: XPCI of a blast wave travelling in a $300 \mu \mathrm{m}$ plastic cylinder (top). Profile of the rectangular cut in grey colour (bottom). Phase contrast enhancement regions are highlighted with green circles while the absorption region of interest (due to the blast wave) is shown in red. 


\section{Chapter V}

\section{Summary of the thesis}

In this thesis we have presented the basics of plasma physics needed for the understanding of generation, transport and applications of the x-ray sources driven by ultra-short (fs) ultra-intense $\left(>10^{18} \mathrm{~W} \cdot \mathrm{cm}^{-2}\right)$ laser pulses.

In the first half of this thesis we described the main mechanisms for electron generation and acceleration in both, under-dense plasmas via Laser Wakefield Acceleration (LWFA) and also in over-critical density plasmas. From one side we have characterized and tested a fully home made, adjustable Kirkpatrick-Baez (KB) microscope in real experimental conditions in which laser driven $\mathrm{X}$-ray sources have been generated. It is noteworthy to mention that the $\mathrm{X}$-ray optics used were unique in terms of mirror technology. A 50nm platinum layer has been used as a coating for two silicon plates of $500 \mu \mathrm{m}$ thickness used as the KB mirrors. We also showed how x-rays, generated from both mechanisms (LWFA and TNSA), in the form of betatron and bremsstrahlung have been transported and/or successfully imaged via the adjustable KB microscope in proton acceleration experiments 1 and 2. On the other side, a few improvements can still be implemented to this KB design such as using bigger mirrors for signal improvements, or modifying the clamping of the mirrors to the bending structure. In addition, it is also possible to replace the micrometer-screw tilt adjustment by piezo-electrical actuators for a fully automated system. Mechanical transducers can also be added to the system for possible future designs.

In this thesis, we have also been able to generate micrometer size laser-driven x-ray sources in order to carry out X-ray Phase Contrast Imaging (XPCI) experiments. We have made possible the use of incoherent X-ray sources, such betatron or bremsstrahlung, to carry out successful XPCI radiography. In the first experiment, LWFA driven betatron radiation has been generated by focusing VEGA-II laser into pure helium gas. The emitted x-rays have been used to carry out XPCI of a micro-structured biological sample. The second experiment, consisted of focusing the PHELIX laser into a $5 \mu \mathrm{m}$ thin tungsten wires to generate bremsstrahlung radiation from a limited source size target. The latter was then used to carry out XPCI radiography of a shock wave (generated by a portion of PHELIX) travelling in high x-ray transmission targets. The successful results obtained from this experiment have proven the possibility of implementing XPCI in future ICF experiments.

The next step would be to implement these limited laser driven x-ray source size targets (e.g. tungsten wire used previously) to experiments at high repetition rate (HRR) facilities for various XPCI applications with the additional ability of improving the statistics. We believe that a parallel development of a suitable target holder for HRR is also required.

Keywords: Kirkpatrick-Baez microscope, X-ray transport, X-ray Phase Contrast Imaging 



\section{Chapter VI}

\section{Resumen de la thesis (Spanish Summary)}

En esta Tesis se han presentado los fundamentos necesarios de la Física de Plasmas para una mejor comprensión de la generación, transporte y aplicaciones de fuentes de rayos $\mathrm{X}$ generadas por pulsos láser ultra-cortos (30 fs) y ultra-intensos $\left(>10^{18} \mathrm{~W} / \mathrm{cm}^{2}\right)$.

En la primera mitad de la Tesis se han descrito los principales mecanismos de la generación y aceleración de electrones en "under dense plasmas" mediante el proceso conocido como Laser Weakfield Acceleration (LWFA) y también en "over dense plasmas" mediante el mecanismo conocido como Target Normal Seath Acceleration (TNSA). Por un lado se ha construido caracterizado y testado un microscopio ajustable Kirkpatrick-Baez (KB) en condiciones experimentales reales mediante fuentes de radiación X generadas por láser. Es importante resaltar que la singularidad de la óptica utilizada en cuanto a la tecnología usada en los espejos. Se ha usado, como "espejos" del KB dos placas de $50 \mathrm{~mm}$ silicio recubiertos de una capa de platino de $50 \mathrm{~nm}$ como recubrimiento. También hemos descrito cómo los rayos $\mathrm{X}$ generados por ambos mecanismos (LWFA y TNSA) en forma de radiación betatron y bremsstrahlung han sido guiados yes ha podido hacer imagen con éxito con ellos mediante el microscopio ajustable KB en los experimentos de aceleración de protones 1 y 2 . Por otra parte hay que decir que algunas mejoras se pueden implementr aun en este diseño de KB tales como usar espejos de mayor superficie para mejorar la señal o modificar la sujeción de los espejos a la estructura de flexión. Además también es posible reemplazar el ajuste del tilt (inclinación) del tornillo micrométrico por un actuador piezo-eleéctrico quedando así el sistema totalmete automatizado. También se pueden añadir transductores mecánicos para futuros diseños.

En esta Tesis también se han conseguido generar fuentes de rayos $\mathrm{X}$ de tamaño de tamaño micrométrico con el fin de aplicarlo en experimentos de Radiografía por Contraste de Fase. Ha sido posible usar fuentes de rayos $\mathrm{X}$ incoherentes tales como Betatrón (espacialmente incoherentes) y Bremsstrahlung para llevar a cabo satisfactoriamente Radiografías por Contraste de Fase.

En el primer experimento, la radiación Betatrón generada mediante LWFA ha sido producida focalizando el láser Vega-2 en un chorro de gas de helio puro. Los rayos X generados han sido usados para hacer Radiografía por Contraste de Fase de una muestra biológica microestructurada. El segundo experimento ha consistido en focalizar el láser PHELIX sobre filamentos de tungsteno de 5 micras de espesor para generar radiación Bremsstrahlung en un blanco con tamaño de fuente limitado. En esta última ha sido usada para realizar Radiografías por Contraste 
de Fase de una onda de choque (generada por una sección del láser PHELIX) viajando a través de blancos (targets) con alta transmisividad de rayos $\mathrm{X}$.

El éxito obtenido en los resultados de esta experimento han confirmado la posibilidad de implementar la técnica de Radiografía por Contraste de Fase un futuros experimentos de Fusión por Confinamiento Inercial (ICF).

El próximo paso podrían ser estas fuentes de rayos $\mathrm{X}$ basadas en target de tamaño reducido (por ejemplo, los filamentos de tungsteno usados anteriormente) en experimentos y en instalaciones donde sea posible disparar a alta tasa de repetición (HRR) para varias aplicaciones en Radiografía por Contraste de Fase facilitando así la estadística. Creemos también que es necesario un desarrollo en paralelo de un soporte de los targets adaptado para trabajar en régimen de la alta tasa de repetición.

Palabras clave: Microscopio Kirkpatrick-Baez, Transporte de rayos X, Imagen por rayos X mediante Contraste de Fase 


\section{Bibliography}

[Antonelli et al., 2019] Antonelli, L., Barbato, F., Mancelli, D., Trela, J., Zeraouli, G., Boutoux, G., Neumayer, P., Atzeni, S., Schiavi, A., Volpe, L., Bagnoud, V., Brabetz, C., Zielbauer, B., Bradford, P., Woolsey, N., Borm, B., and Batani, D. (2019). X-ray phase-contrast imaging for laser-induced shock waves. EPL (Europhysics Letters), 125(3):35002.

[Antonelli et al., 2015] Antonelli, L., Forestier-Colleoni, P., Folpini, G., Bouillaud, R., Faenov, A., Fedeli, L., Fourment, C., Giuffrida, L., Hulin, S., Pikuz, S., Santos, J. J., Volpe, L., and Batani, D. (2015). Measurement of reflectivity of spherically bent crystals using ka signal from hot electrons produced by laser-matter interaction. Review of Scientific Instruments, 86(7):073507.

[Baez, 1961] Baez, A. V. (1961). Fresnel zone plate for optical image formation using extreme ultraviolet and soft x radiation. Journal of the Optical Society of America, 51:4.

[Bandres and Gutiérrez-Vega, 2004] Bandres, M. A. and Gutiérrez-Vega, J. C. (2004). Incegaussian beams. Opt. Lett., 29(2):144-146.

[Barbato et al., 2019a] Barbato, F., Batani, D., Mancelli, D., Trela, J., Zeraouli, G., Boutoux, G., Neumayer, P., Atzeni, S., Schiavi, A., Volpe, L., Bagnoud, V., Brabetz, C., Zielbauer, B., Bradford, P., Woolsey, N., Borm, B., and Antonelli, L. (2019a). Propagation-based imaging phase-contrast enhanced imaging setup for single shot acquisition using laser-generated $\mathrm{x}$-ray sources. Journal of Instrumentation, 14(03):C03005-C03005.

[Barbato et al., 2019b] Barbato, F., Batani, D., Mancelli, D., Trela, J., Zeraouli, G., Boutoux, G., Neumayer, P., Atzeni, S., Schiavi, A., Volpe, L., Bagnoud, V., Brabetz, C., Zielbauer, B., Bradford, P., Woolsey, N., Borm, B., and Antonelli, L. (2019b). Quantitative phase contrast imaging of a shock-wave with a laser-plasma based x-ray source. Scientific Reports, 9(118805):2045-2322.

[Batani et al., 2018] Batani, D., d'Humieres, E., Santos, J., and Tikhonchuk, V. (2018). Plasma physics theory and experiments. PowerLaPs, pages O1 / LTT-C1.

[Beg et al., 1997] Beg, F. N., Bell, A. R., Dangor, A. E., Danson, C. N., Fews, A. P., Glinsky, M. E., Hammel, B. A., Lee, P., Norreys, P. A., and Tatarakis, M. (1997). A study of picosecond laser-solid interactions up to. Physics of Plasmas, 4(2):447-457.

[Bennett and Porteus, 1961] Bennett, H. E. and Porteus, J. O. (1961). Relation between surface roughness and specular reflectance at normal incidence. J. Opt. Soc. Am., 51(2):123-129.

[Bollig et al., 1995] Bollig, C., Clarkson, W. A., and Hanna, D. C. (1995). Stable highrepetition-rate single-frequency q-switched operation by feedback suppression of relaxation oscillation. Opt. Lett., 20(12):1383-1385. 
[Bryzek, 1991] Bryzek, J. (1991). Silicon sensors and microstructures. In Electro International, 1991, pages 661-665.

[Casnati et al., 1982] Casnati, E., Tartari, A., and Baraldi, C. (1982). An empirical approach to k-shell ionisation cross section by electrons. Journal of Physics B: Atomic and Molecular Physics, 15(1):155-167.

[Champeaux, 2006] Champeaux, J. P. (2006). Systèmes d'imagerie à haute résolution dans le domaine des rayons x pour le laser mégajoule. Journal de Physique IV France, 138:285-295.

[Clark et al., 2000] Clark, E. L., Krushelnick, K., Davies, J. R., Zepf, M., Tatarakis, M., Beg, F. N., Machacek, A., Norreys, P. A., Santala, M. I. K., Watts, I., and Dangor, A. E. (2000). Measurements of energetic proton transport through magnetized plasma from intense laser interactions with solids. Phys. Rev. Lett., 84:670-673.

[CLPU, 2020] CLPU (2020). See. www.clpu.es.

[Corde et al., 2013] Corde, S., Ta Phuoc, K., Lambert, G., Fitour, R., Malka, V., Rousse, A., Beck, A., and Lefebvre, E. (2013). Femtosecond x rays from laser-plasma accelerators. Rev. Mod. Phys., 85:1-48.

[CXRO, 2019] CXRO (2019). See. henke.lbl.gov/opticalconstants.

[Diemoz et al., 2012] Diemoz, P. C., Bravin, A., and Coan, P. (2012). Theoretical comparison of three $\mathrm{x}$-ray phase-contrast imaging techniques: propagation-based imaging, analyzer-based imaging and grating interferometry. Opt. Express, 20(3):2789-2805.

[Eberhardt, 2015] Eberhardt, W. (2015). Synchrotron radiation: A continuing revolution in xray science-diffraction limited storage rings and beyond. Journal of Electron Spectroscopy and Related Phenomena, 200:31 - 39. Special Anniversary Issue: Volume 200.

[Esarey et al., 1997] Esarey, E., Hubbard, R. F., Leemans, W. P., Ting, A., and Sprangle, P. (1997). Electron injection into plasma wakefields by colliding laser pulses. Phys. Rev. Lett., 79:2682-2685.

[Esarey and Pilloff, 1995] Esarey, E. and Pilloff, M. (1995). Trapping and acceleration in nonlinear plasma waves. Physics of Plasmas, 2(5):1432-1436.

[Esarey et al., 2009] Esarey, E., Schroeder, C. B., and Leemans, W. P. (2009). Physics of laserdriven plasma-based electron accelerators. Rev. Mod. Phys., 81:1229-1285.

[Faure, 2016] Faure, J. (2016). Plasma Injection Schemes for Laser-Plasma Accelerators. (arXiv:1705.09584):143 p. CERN Yellow Reports.

[Fourmaux et al., 2011] Fourmaux, S., Corde, S., Phuoc, K. T., Lassonde, P., Lebrun, G., Payeur, S., Martin, F., Sebban, S., Malka, V., Rousse, A., and Kieffer, J. C. (2011). Single shot phase contrast imaging using laser-produced betatron x-ray beams. Opt. Lett., 36(13):2426-2428.

[Geddes et al., 2008] Geddes, C. G. R., Nakamura, K., Plateau, G. R., Toth, C., Cormier-Michel, E., Esarey, E., Schroeder, C. B., Cary, J. R., and Leemans, W. P. (2008). Plasma-densitygradient injection of low absolute-momentum-spread electron bunches. Phys. Rev. Lett., 100:215004. 
[Geloni et al., 2010] Geloni, G., Saldin, E., Samoylova, L., Schneidmiller, E., Sinn, H., Tschentscher, T., and Yurkov, M. (2010). Coherence properties of the european XFEL. New Journal of Physics, 12(3):035021.

[Gibbon, 2005] Gibbon, P. (2005). Short pulse laser interaction with matter. Imperial College Press.

[Glenzer et al., 2010] Glenzer, S. H., MacGowan, B. J., Michel, P., Meezan, N. B., Suter, L. J., Dixit, S. N., Kline, J. L., Kyrala, G. A., Bradley, D. K., Callahan, D. A., Dewald, E. L., Divol, L., Dzenitis, E., Edwards, M. J., Hamza, A. V., Haynam, C. A., Hinkel, D. E., Kalantar, D. H., Kilkenny, J. D., Landen, O. L., Lindl, J. D., LePape, S., Moody, J. D., Nikroo, A., Parham, T., Schneider, M. B., Town, R. P. J., Wegner, P., Widmann, K., Whitman, P., Young, B. K. F., Van Wonterghem, B., Atherton, L. J., and Moses, E. I. (2010). Symmetric inertial confinement fusion implosions at ultra-high laser energies. Science, 327(5970):1228-1231.

[Haines et al., 2009] Haines, M. G., Wei, M. S., Beg, F. N., and Stephens, R. B. (2009). Hotelectron temperature and laser-light absorption in fast ignition. Phys. Rev. Lett., 102:045008.

[Harvey et al., 2012] Harvey, J. E., Choi, N., Schroeder, S., and Duparré, A. (2012). Total integrated scatter from surfaces with arbitrary roughness, correlation widths, and incident angles. Optical Engineering, 51(1):1 - 12.

[Henke et al., 1993] Henke, B., Gullikson, E., and Davis, J. (1993). X-ray interactions: Photoabsorption, scattering, transmission, and reflection at $\mathrm{e}=50-30,000 \mathrm{ev}, \mathrm{z}=1-92$. Atomic Data and Nuclear Data Tables, 54(2):181 - 342.

[Hochberg and Baehr-Jones, 2010] Hochberg, M. and Baehr-Jones, T. (2010). Silicon photonic devices can be built using commercial cmos chip fabrication facilities, or 'fabs'. however, nearly all research groups continue to design, build and test chips internally, rather than leveraging shared cmos foundry infrastructure. Nature Photonics, 4(8):492-494.

[Hombourger, 1998] Hombourger, C. (1998). An empirical expression for k-shell ionization cross section by electron impact. Journal of Physics B: Atomic, Molecular and Optical Physics, 31(16):3693-3702.

[Hong et al., 2016] Hong, X., Ehm, L., Zhong, Z., Ghose, S., Duffy, T. S., and Weidner, D. J. (2016). High-energy X-ray focusing and applications to pair distribution function investigation of Pt and Au nanoparticles at high pressures. (2045-2322):21434. Scientific Reports.

[Howells, 2000] Howells (2000). Theory and practice of elliptically bent x-ray mirrors. Optical Engineering, 39:10.

[Huba, 2013] Huba, J. D. (2013). NRL PLASMA FORMULARY Supported by The Office of Naval Research. Naval Research Laboratory, Washington, DC.

[Iida and Hirano, 1996] Iida, A. and Hirano, K. (1996). Kirkpatrick-baez optics for a sub-um synchrotron x-ray microbeam and its applications to x-ray analysis. Nuclear Instruments and Methods in Physics Research Section B: Beam Interactions with Materials and Atoms, 114(1):149 - 153 .

[Jackson, 1962] Jackson, J. D. (1962). Classical electrodynamics, 2nd edition. J. Wiley. 
[Joshi et al., 1984] Joshi, C., Mori, W. B., Dawson, J. M., Kindel, J. M., and Forslund, D. W. (1984). Ultrahigh gradient particle acceleration by intense laser-driven plasma density waves, rather than leveraging shared cmos foundry infrastructure. Nature, 311(5986):525-529.

[Kahoul et al., 2011] Kahoul, A., Abassi, A., Deghfel, B., and Nekkab, M. (2011). K-shell fluorescence yields for elements with $6<\mathrm{z}<99$. Radiation Physics and Chemistry, pages 369377.

[Kirkpatrick and Baez, 1948] Kirkpatrick, P. and Baez, A. V. (1948). Formation of optical images by x-rays. Journal of the Optical Society of America, 38:9.

[Kneip et al., 2011] Kneip, S., McGuffey, C., Dollar, F., Bloom, M. S., Chvykov, V., Kalintchenko, G., Krushelnick, K., Maksimchuk, A., Mangles, S. P. D., Matsuoka, T., Najmudin, Z., Palmer, C. A. J., Schreiber, J., Schumaker, W., Thomas, A. G. R., and Yanovsky, V. (2011). X-ray phase contrast imaging of biological specimens with femtosecond pulses of betatron radiation from a compact laser plasma wakefield accelerator. Applied Physics Letters, 99(9):093701.

[Koepf et al., 2002] Koepf, G. A., Marshalek, R. G., and Begley, D. L. (2002). Space laser communications: A review of major programs in the united states. AEU - International Journal of Electronics and Communications, 56(4):232 - 242.

[Krall et al., 1994] Krall, J., Esarey, E., Sprangle, P., and Joyce, G. (1994). Propagation of radius-tailored laser pulses over extended distances in a uniform plasma*. Physics of Plasmas, 1(5):1738-1743.

[Krause, 1979] Krause, M. O. (1979). Atomic radiative and radiationless yields for k and 1 shells. Journal of Physical and Chemical Reference Data, 8(2):307-327.

[Krenkel et al., 2015] Krenkel, M., Markus, A., Bartels, M., Dullin, C., Alves, F., and Salditt, T. (2015). Phase-contrast zoom tomography reveals precise locations of macrophages in mouse lungs. (2045-2322):9973. Scientific Reports.

[Kruer, 1988] Kruer, W. (1988). The physics of laser plasma interactions. Frontiers in physics. Addison-Wesley.

[Legall et al., 2006] Legall, H., Stiel, H., Arkadiev, V., and Bjeoumikhov, A. (2006). High spectral resolution x-ray optics with highly oriented pyrolytic graphite. Opt. Express, 14(10):4570-4576.

[Lu et al., 2007] Lu, W., Tzoufras, M., Joshi, C., Tsung, F. S., Mori, W. B., Vieira, J., Fonseca, R. A., and Silva, L. O. (2007). Generating multi-gev electron bunches using single stage laser wakefield acceleration in a 3d nonlinear regime. Phys. Rev. ST Accel. Beams, 10:061301.

[Maddox et al., 2011] Maddox, B. R., Park, H. S., Remington, B. A., Izumi, N., Chen, S., Chen, C., Kimminau, G., Ali, Z., Haugh, M. J., and Ma, Q. (2011). High-energy x-ray backlighter spectrum measurements using calibrated image plates. Review of Scientific Instruments, 82(2):023111.

[Maiman, 1960] Maiman, T. H. (1960). Stimulated optical radiation in ruby. Nature, 187:493494. 
[Maksimchuk et al., 2000] Maksimchuk, A., Gu, S., Flippo, K., Umstadter, D., and Bychenkov, V. Y. (2000). Forward ion acceleration in thin films driven by a high-intensity laser. Phys. Rev. Lett., 84:4108-4111.

[Malka and Miquel, 1996] Malka, G. and Miquel, J. L. (1996). Experimental confirmation of ponderomotive-force electrons produced by an ultrarelativistic laser pulse on a solid target. Phys. Rev. Lett., 77:75-78.

[Malka, 2012] Malka, V. (2012). Laser plasma accelerators. Physics of Plasmas, 19(5):055501.

[Mangles et al., 2012] Mangles, S. P. D., Genoud, G., Bloom, M. S., Burza, M., Najmudin, Z., Persson, A., Svensson, K., Thomas, A. G. R., and Wahlström, C.-G. (2012). Self-injection threshold in self-guided laser wakefield accelerators. Phys. Rev. ST Accel. Beams, 15:011302.

[Mangles et al., 2004] Mangles, S. P. D., Murphy, C. D., Najmudin, Z., Thomas, A. G. R., Collier, J. L., Dangor, A. E.and Divall, E. J., Foster, P. S., Gallacher, J. G., Hooker, C. J., Jaroszynski, D. A., Langley, A. J., Mori, W. B., Norreys, P. A., Tsung, F. S., Viskup, R., Walton, B. R., and Krushelnick, K. (2004). Monoenergetic beams of relativistic electrons from intense laser-plasma interactions. Nature, 431:535-538.

[Marshall, 2017] Marshall, F. (2017). A framed, 16-image kirkpatrick-baez x-ray microscope. Review of Scientific Instruments, 88.

[Martinez de la Ossa et al., 2015] Martinez de la Ossa, A., Mehrling, T. J., Schaper, L., Streeter, M. J. V., and Osterhoff, J. (2015). Wakefield-induced ionization injection in beam-driven plasma accelerators. Physics of Plasmas, 22(9):093107.

[Masters, 2004] Masters, B. R. (2004). Lasers in ophthalmology basic, diagnostic and surgical aspects. Springer-Verlag, page 242:446.

[Michette, 1986] Michette, A. G. (1986). Optical systems for soft x-rays. Plenum Press New York.

[Méndez et al., 2019] Méndez, C., Varela, O., García, E., Hernández, I., Ajates, J., Pisonero, J. D., Sagredo, J. L., Olivar, M., and Roso, L. (2019). VEGA laser facility beamlines management for pump-probe experiments. In Costa, M. F. P. C. M. M., editor, Fourth International Conference on Applications of Optics and Photonics, volume 11207, pages 431 - 436. International Society for Optics and Photonics, SPIE.

[Mo et al., 2013] Mo, M. Z., Chen, Z., Fourmaux, S., Saraf, A., Otani, K., Kieffer, J. C., Tsui, Y. Y., Ng, A., and Fedosejevs, R. (2013). Laser wakefield generated x-ray probe for femtosecond time-resolved measurements of ionization states of warm dense aluminum. Review of Scientific Instruments, 84(12):123106.

[Mocker and Collins, 1965] Mocker, H. W. and Collins, R. J. (1965). Mode competition and self-locking effects in a q-switched ruby laser. Applied Physics Letters, 7(10):270-273.

[Morozov, 2013] Morozov, A. I. (2013). Introduction to plasma dynamics. CRC Press.

[Moseley, 1913] Moseley, H. (1913). Philosophical Magazine, (26):1024. 
[Neumayer et al., 2010] Neumayer, P., Aurand, B., Basko, M., Ecker, B., Gibbon, P., Hochhaus, D. C., Karmakar, A., Kazakov, E., Kühl, T., Labaune, C., Rosmej, O. Tauschwitz, A., Zielbauer, B., and Zimmer, D. (2010). The role of hot electron refluxing in laser-generated k-alpha sources. Physics of Plasmas, 17(10):103103.

[Neumayer et al., 2005] Neumayer, P., Bock, R., Borneis, S., Brambrink, E., Brand, H., Caird, J., Campbell, E., Gaul, E., Goette, S., Haefner, C., and et al. (2005). Status of phelix laser and first experiments. Laser and Particle Beams, 23(3):385-389.

[Paschotta et al., 2002] Paschotta, R., Häring, R., Garnache, A., Hoogland, S., Tropper, A., and Keller, U. (2002). Soliton-like pulse-shaping mechanism in passively mode-locked surfaceemitting semiconductor lasers. Applied Physics B, 75(4):445-451.

[Passoni, 2010] Passoni, M. Bertagna, L. Z. A. (2010). Target normal sheath acceleration: theory, comparison with experiments and future perspectives. New Journal of Physics, 12(4):045012.

[Passoni et al., 2013] Passoni, M., Perego, C., Sgattoni, A., and Batani, D. (2013). Advances in target normal sheath acceleration theory. Physics of Plasmas, 20:060701.

[Pickworth, 2016] Pickworth, L. (2016). The national ignition facility modular kirkpatrick-baez microscope. Review of Scientific Instruments, 87.

[Protopapas et al., 1997] Protopapas, M., Keitel, C. H., and Knight, P. L. (1997). Atomic physics with super-high intensity lasers. Reports on Progress in Physics, 60(4):389-486.

[Quesnel and Mora, 1998] Quesnel, B. and Mora, P. (1998). Theory and simulation of the interaction of ultraintense laser pulses with electrons in vacuum. Phys. Rev. E, 58:3719-3732.

[Rödel et al., 2011] Rödel, C., Heyer, M., Behmke, M., Kübel, M., Jäckel, O., Ziegler, W., Ehrt, D., Kaluza, M. C., and Paulus, G. G. (2011). igh repetition rate plasma mirror for temporal contrast enhancement of terawatt femtosecond laser pulses by three orders of magnitude. Applied Physics B, 103(295-302).

[Roling et al., 2014] Roling, S., Braun, S., Gawlitza, P., Wöstmann, M., Ziegler, E., and Zacharias, H. (2014). Tunable two-color hard x-ray multilayer bragg mirrors. Opt. Lett., 39(9):2782-2785.

[Rosenzweig et al., 1988] Rosenzweig, J. B., Cline, D. B., Cole, B., Figueroa, H., Gai, W., Konecny, R., Norem, J., Schoessow, P., and Simpson, J. (1988). Experimental observation of plasma wake-field acceleration. Phys. Rev. Lett., 61:98-101.

[Roth and Schollmeier, 2016] Roth, M. and Schollmeier, M. (2016). Ion Acceleration - Target Normal Sheath Acceleration. (arXiv:1705.10569):40 p. 40 pages, contribution to the CAS CERN Accelerator School: Plasma Wake Acceleration, CERN, Geneva, Switzerland, 23 - 29 Nov 2014.

[Saleh and Teich, 2007] Saleh, B. and Teich, M. C. (2007). Fundamentals of Photonics. J. Wiley.

[Salzmann et al., 2002] Salzmann, D., Reich, C., Uschmann, I., Forster, E., and Gibbon, P. (2002). Theory of $\mathrm{k}$ alpha generation by femtosecond laser-produced hot electrons in thin foils. Phys. Rev. E, 65:036402. 
[Sansonetti and Martin, 2005] Sansonetti, J. E. and Martin, W. C. (2005). Handbook of basic atomic spectroscopic data. Journal of Physical and Chemical Reference Data, 34(4):15592259.

[Sawada et al., 2014] Sawada, H., Wei, M. S., Chawla, S., Morace, A., Akli, K., Yabuuchi, T., Nakanii, N., Key, M. H., Patel, P. K., Mackinnon, A. J., McLean, H. S., Stephens, R. B., and Beg, F. N. (2014). Investigation of fast-electron-induced ka $x$ rays in laser-produced blow-off plasma. Phys. Rev. E, 89:033105.

[Schlenvoigt et al., 2008] Schlenvoigt, H., Haupt, K., Debus, A., Budde, F., Jackel, O., Pfotenhauer, S., Schwoerer, H. Rohwer, E., Gallacher, J. G., and Brunetti, E. Shanks, R. P. W. S. M. J. D. A. (2008). A compact synchrotron radiation source driven by a laser-plasma wakefield accelerator. Nature, 4:130-133.

[Schönlein et al., 2016] Schönlein, A., Boutoux, G., Pikuz, S., Antonelli, L., Batani, D., Debayle, A., Franz, A., Giuffrida, L., Honrubia, J. J., Jacoby, J., Khaghani, D., Neumayer, P., Rosmej, O. N., Sakaki, T., Santos, J. J., and Sauteray, A. (2016). Generation and characterization of warm dense matter isochorically heated by laser-induced relativistic electrons in a wire target. EPL (Europhysics Letters), 114(4):45002.

[Schwoerer et al., 2006] Schwoerer, H., Pfotenhauer, S., and O.Jäckel (2006). Laser-plasma acceleration of quasi-monoenergetic protons from microstructured targets. Nature, 439(14764687).

[Sentoku et al., 2003] Sentoku, Y., Cowan, T. E., Kemp, A., and Ruhl, H. (2003). High energy proton acceleration in interaction of short laser pulse with dense plasma target. Physics of Plasmas, 10(5):2009-2015.

[Shadwick et al., 2009] Shadwick, B. A., Schroeder, C. B., and Esarey, E. (2009). Nonlinear laser energy depletion in laser-plasma accelerators. Physics of Plasmas, 16(5):056704.

[Sokollik, 2011] Sokollik, T. (2011). Investigations of field dynamics in laser plasmas with proton imaging. Springer-Verlag Berlin Heidelberg.

[Spiegel, 1995] Spiegel, P. K. (1995). The first clinical x-ray made in america-100 years. American Journal of Roentgenology, 164(1):0361-803X.

[Sprangle et al., 1990] Sprangle, P., Esarey, E., and Ting, A. (1990). Nonlinear interaction of intense laser pulses in plasmas. Phys. Rev. A, 41:4463-4469.

[Strickland and Mourou, 1985] Strickland, D. and Mourou, G. (1985). Compression of amplified chirped optical pulses. Optics Communications, 56(3):219 - 221.

[Tajima and Dawson, 1979] Tajima, T. and Dawson, J. M. (1979). Laser electron accelerator. Phys. Rev. Lett., 43:267-270.

[Thaury et al., 2007] Thaury, C .and Quéré, F., Geindre, J.-P., Levy, A., Ceccotti, T., Monot, P., Bougeard, M., Réau, F., d'Oliveira, P., Audebert, P., Marjoribanks, R., and Martin, P. (2007). Plasma mirrors for ultrahigh-intensity optics. Nature Physics, 3(424-429).

[Thompson et al., 2017] Thompson, A. R., Moran, J. M., and Swenson, G. W. (2017). Van Cittert-Zernike Theorem, Spatial Coherence, and Scattering, pages 767-786. Springer International Publishing, Cham. 
[Volpe et al., 2013] Volpe, L., Batani, D., Morace, A., and Santos, J. J. (2013). Collisional and collective effects in two dimensional model for fast-electron transport in refluxing regime. Physics of Plasmas, 20(1):013104.

[Volpe et al., 2019] Volpe, L., Fedosejevs, R., Gatti, G., Pérez-Hernández, J. A., Méndez, C., Apiñaniz, J., Vaisseau, X., Salgado, C., Huault, M., and Malko, S. (2019). Generation of high energy laser-driven electron and proton sources with the $200 \mathrm{tw}$ system vega 2 at the centro de laseres pulsados. High Power Laser Science and Engineering, 7:e25.

[Wilks et al., 1992] Wilks, S. C., Kruer, W. L., Tabak, M., and Langdon, A. B. (1992). Absorption of ultra-intense laser pulses. Phys. Rev. Lett., 69:1383-1386.

[Wu and Liu, 2003] Wu, X. and Liu, H. (2003). A general theoretical formalism for X-ray phase contrast imaging. Journal of X-ray science and technology, 11:33-42.

[x-ray data boklet, ] x-ray data boklet. See. http://xdb.lbl.gov/Section1.html.

[XOP, 2019] XOP (2019). See. www.esrf.eu/Instrumentation/software/data-analysis/xop2.4.

[Zeraouli et al., 2019] Zeraouli, G., Gatti, G., Longman, A., Pérez-Hernández, J. A., Arana, D., Batani, D., Jakubowska, K., Volpe, L., Roso, L., and Fedosejevs, R. (2019). Development of an adjustable kirkpatrick-baez microscope for laser driven x-ray sources. Review of Scientific Instruments, 90(6):063704. 